University of Tennessee Health Science Center UTHSC Digital Commons

\title{
H5N1 Influenza Virus Induces a Parkinsonian Pathology
}

Haeman Jang

University of Tennessee Health Science Center

Follow this and additional works at: https://dc.uthsc.edu/dissertations

Part of the Neurosciences Commons

\section{Recommended Citation}

Jang, Haeman , "H5N1 Influenza Virus Induces a Parkinsonian Pathology" (2010). Theses and Dissertations (ETD). Paper 115. http://dx.doi.org/10.21007/etd.cghs.2010.0151.

This Dissertation is brought to you for free and open access by the College of Graduate Health Sciences at UTHSC Digital Commons. It has been accepted for inclusion in Theses and Dissertations (ETD) by an authorized administrator of UTHSC Digital Commons. For more information, please contact jwelch30@uthsc.edu. 


\title{
H5N1 Influenza Virus Induces a Parkinsonian Pathology
}

\begin{abstract}
The greatest threat for an influenza pandemic at this time is posed by the highly pathogenic H5N1 avian influenza virus. To date, $63 \%$ of the 436 known human cases of H5N1 infection have proven fatal. Animals infected by $\mathrm{H} 5 \mathrm{~N} 1$ viruses have demonstrated acute neurological signs ranging from mild encephalitis to motor disturbances and coma. However, no studies have examined the longer-term neurologic consequences of H5N1 infection. We show that this virus travels from the peripheral nervous system into the central nervous system (CNS) to higher levels of the neuroaxis, using C57BL/6J mice that are infected by the A/VN/1203/04 H5N1 virus (without adaptation). In regions infected by H5N1 virus, we observe activation of microglia and alpha-synuclein phosphorylation and aggregation that persists long after resolution of the infection.

To determine if the pathology worsened with age, we examined: 1) substantia nigra pars compacta $(\mathrm{SNpc})$ tyrosine hydroxylase positive dopaminergic neuron number and striatal dopamine and its metabolites contents through 90 days post infection (dpi), 2) examined the inflammatory effect of infection by quantitatively measuring the total number of resting and activated microglia in the SNpc and then examined the production of cytokines in regions of the brain infected by H5N1. We found that infection with $\mathrm{H} 5 \mathrm{~N} 1$ induces a reversible reduction of both the number of dopaminergic neurons in the SNpc and the amount of dopamine and its metabolites in the striatum. Examination of other indolamines demonstrated a significant and sustained reductionof serotonin in regions of the brain infected with H5N1. We also observed that areas of the brain infected with H5N1 expressed altered levels of proinflammatory cytokines, chemokines and growth factors.

We examined if H5N1 priming potentiates paraquat (PQ) induced neurotoxicity in the basal ganglia. We found that $\mathrm{H} 5 \mathrm{~N} 1$ priming did not increase the sensitivity of C57BL/6J mice to intraperitoneal (ip) administration of paraquat. Rather, H5N1 priming protects the dopaminergic neurons from PQ-induced neurodegeneration and diminishes immune response produced by PQ in the CNS.
\end{abstract}

\section{Document Type}

Dissertation

Degree Name

Doctor of Philosophy (PhD)

Program

Biomedical Sciences

Research Advisor

Richard J. Smeyne, Ph.D.

\section{Keywords}

alpha-synuclein, encephalitis, influenza, neurodegeneration, neuroinflammation, Parkinson's disease

\section{Subject Categories}

Medicine and Health Sciences | Neurosciences 


\title{
H5N1 INFLUENZA VIRUS INDUCES A PARKINSONIAN PATHOLOGY
}

\author{
A Dissertation \\ Presented for \\ The Graduate Studies Council \\ The University of Tennessee \\ Health Science Center
}

In Partial Fulfillment

Of the Requirements for the Degree

Doctor of Philosophy

From The University of Tennessee

By

Haeman Jang

May 2010 
Portion of Chapter 1 (C) 2008 by Elsevier B.V. Chapter 2 (C) 2009 by the National Academy of Sciences

All other material (C) 2010 by Haeman Jang 


\section{DEDICATION}

This dissertation is dedicated to

my parents, Taek Hyun Jang and Hyun Nam Kyung,

my wife, Jae Young Um and my daughter, Ashley Jang and my son, Spencer Jang for their endless love and support. 


\section{ACKNOWLEDGEMENTS}

I would like to appreciate Dr. Richard J. Smeyne for his support, patience, guidance and encouragement he has given to me. I extend my gratitude to my committee members, Dr. Kristine M. Hamre, Dr. Michael C. Levin, Dr. James I. Morgan, Dr. Richard J. Webby for their advice and suggestions.

I would also like to show my appreciation to all the members of the Smeyne lab their valuable advice and informative discussions and friendship. In particular, I thank to Dr. Yun Jiao, Dr. Zachary Baquet, and Michelle Smeyne for their teaching and advice in animal experiments, Dr. Shankar Sadasivan and Dr. Ane Korff for their informative discussion and friendship, Dr. Amar Pani for running HPLC, Dr. David Boltz for his dedicated hard work and effort for collaboration.

I want to acknowledge grant support of Michael J. Fox Foundation and National Institutes of Health Grant R21NS058310 (to R.J.S.), and the American Lebanese Syrian Associated Charities. 


\begin{abstract}
The greatest threat for an influenza pandemic at this time is posed by the highly pathogenic H5N1 avian influenza virus. To date, $63 \%$ of the 436 known human cases of H5N1 infection have proven fatal. Animals infected by H5N1 viruses have demonstrated acute neurological signs ranging from mild encephalitis to motor disturbances and coma. However, no studies have examined the longer-term neurologic consequences of H5N1 infection. We show that this virus travels from the peripheral nervous system into the central nervous system (CNS) to higher levels of the neuroaxis, using C57BL/6J mice that are infected by the A/VN/1203/04 H5N1 virus (without adaptation). In regions infected by $\mathrm{H} 5 \mathrm{~N} 1$ virus, we observe activation of microglia and alpha-synuclein phosphorylation and aggregation that persists long after resolution of the infection.
\end{abstract}

To determine if the pathology worsened with age, we examined: 1) substantia nigra pars compacta (SNpc) tyrosine hydroxylase positive dopaminergic neuron number and striatal dopamine and its metabolites contents through 90 days post infection (dpi), 2) examined the inflammatory effect of infection by quantitatively measuring the total number of resting and activated microglia in the SNpc and then examined the production of cytokines in regions of the brain infected by H5N1. We found that infection with $\mathrm{H} 5 \mathrm{~N} 1$ induces a reversible reduction of both the number of dopaminergic neurons in the $\mathrm{SNpc}$ and the amount of dopamine and its metabolites in the striatum. Examination of other indolamines demonstrated a significant and sustained reduction of serotonin in regions of the brain infected with $\mathrm{H} 5 \mathrm{~N} 1$. We also observed that areas of the brain infected with $\mathrm{H} 5 \mathrm{~N} 1$ expressed altered levels of pro-inflammatory cytokines, chemokines and growth factors.

We examined if $\mathrm{H} 5 \mathrm{~N} 1$ priming potentiates paraquat (PQ) induced neurotoxicity in the basal ganglia. We found that $\mathrm{H} 5 \mathrm{~N} 1$ priming did not increase the sensitivity of C57BL/6J mice to intraperitoneal (ip) administration of paraquat. Rather, H5N1 priming protects the dopaminergic neurons from PQ-induced neurodegeneration and diminishes immune response produced by PQ in the CNS. 


\section{TABLE OF CONTENTS}

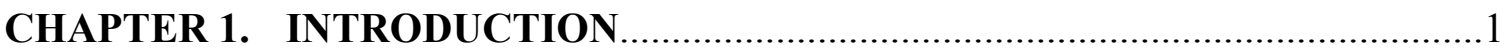

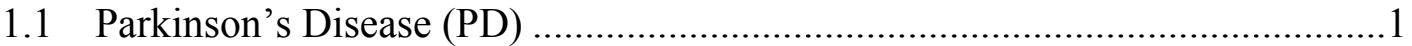

1.2 Functional Neuroanatomy Underlying PD ....................................................

1.2.1 Basal ganglia ................................................................................

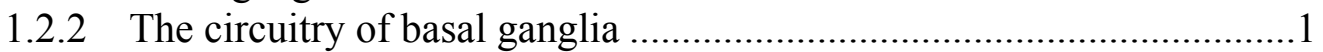

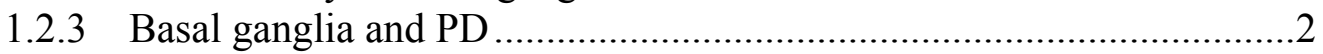

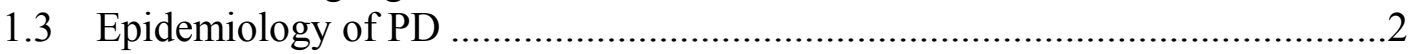

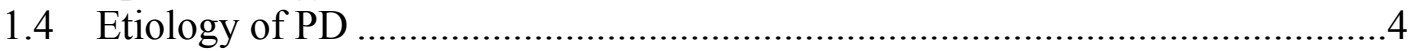

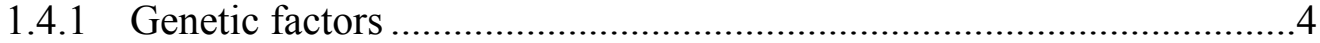

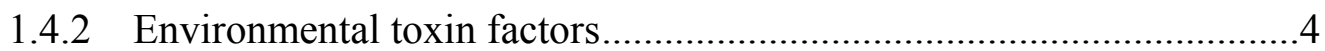

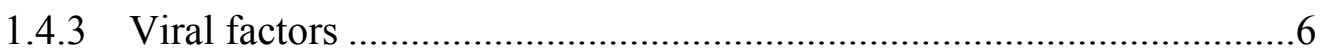

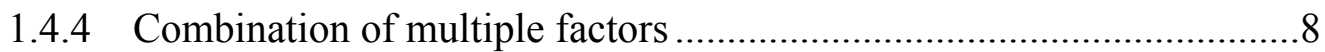

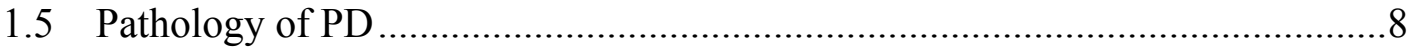

1.5.1 The role of Lewy bodies (LB) ………........................................

1.5.2 The structure and function of $\alpha$-synuclein ............................................

1.5.3 Association of $\alpha$-synuclein with the formation of LB in PD ...............10

1.5.4 Braak's pathological observations ....................................................11

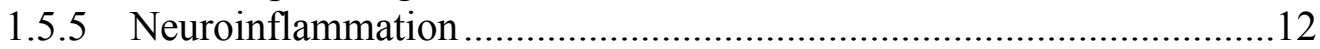

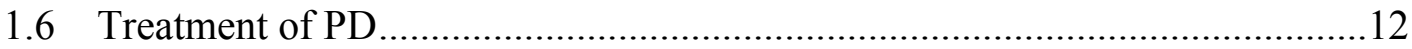

1.6.1 Pharmacological treatments ..............................................................12

1.6.2 Surgical treatments and deep brain stimulation ...................................13

1.6.3 Human mesencephalic tissue transplantation ......................................13

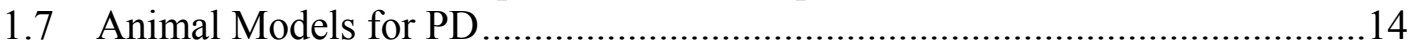

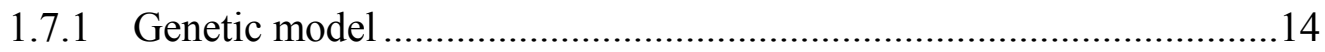

1.7.1.1 $\alpha$-synuclein .....................................................................14

1.7.1.2 Leucine-rich repeat kinase (LRRK) 2 ………………….......15

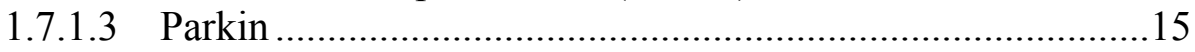

1.7.2 Neurotoxin models......................................................................... 16

1.7.2.1 1-Methyl-4-phenyl-1,2,3,6-tetrahydropyridine (MPTP).......16

1.7.2.2 6-Hydroxydopamine (OHDA) ............................................17

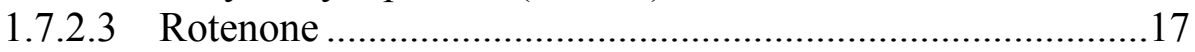

1.7.2.4 Paraquat and maneb ...........................................................17

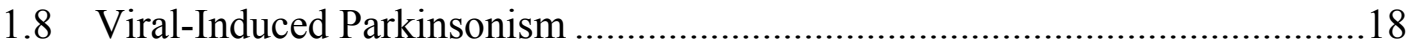

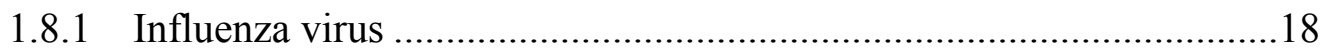

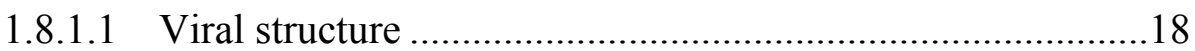

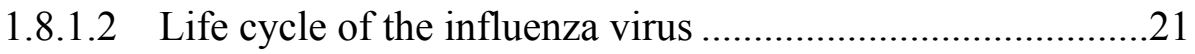

1.8.2 Previous human influenza pandemics..................................................22

1.8.3 Von Economo's disease and postencephalic parkinsonism..................22

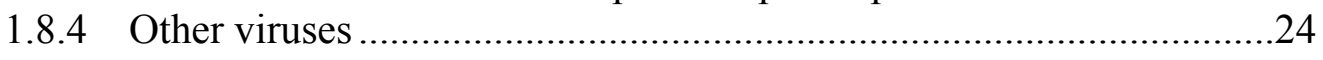

1.8.4.1 Coxsackie virus.................................................................24

1.8.4.2 Japanese encephalitis B, St. Louis and West Nile viruses....25

1.8.4.3 Human immunodeficiency virus (HIV) ................................26

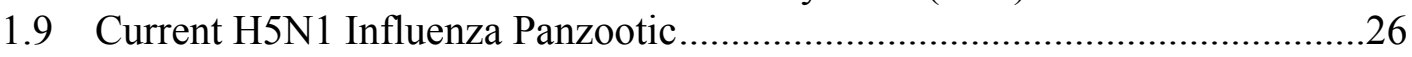


1.9.1 Evidence of neurotropism ........................................................27

1.9.2 The possible routes of entry into the central nervous system (CNS)...27

1.9.3 Scope and objective of dissertation..................................................28

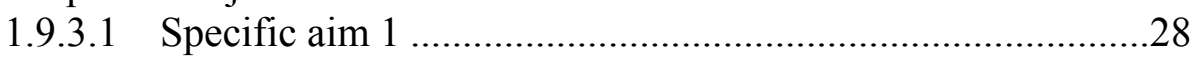

1.9.3.2 Specific aim 2 .....................................................28

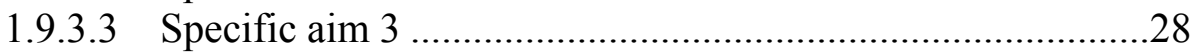

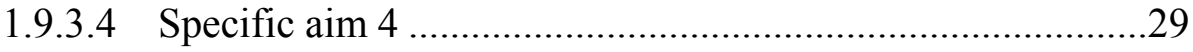

CHAPTER 2. HIGHLY PATHOGENIC H5N1 INFLUENZA VIRUS CAN ENTER THE CENTRAL NERVOUS SYSTEM AND INDUCE NEUROINFLAMMATION AND NEURODEGENERATION .......30

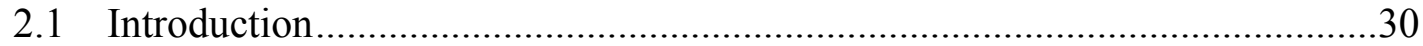

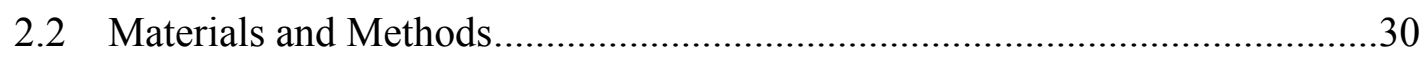

2.2.1 Virus stock preparation and inoculation of mice with $\mathrm{H} 5 \mathrm{~N} 1$..............30

2.2.2 Immunocytochemistry ........................................................... 31

2.2.3 Dorsal root ganglia (DRG) neuron culture and microfluidic

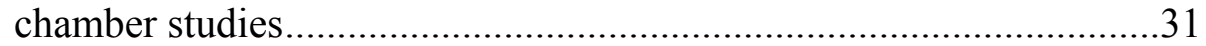

2.2.4 Quantification of phosphorylated $\alpha$-synuclein expression..................32

2.2.5 Stereological assessment of SNpc dopaminergic neuron number .......33

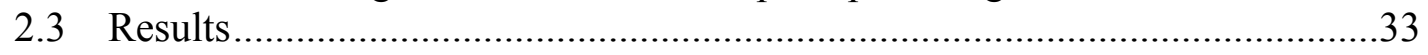

2.3.1 Body weight loss in H5N1 virus infected mice ...............................33

2.3.2 Spatial and temporal distribution of H5N1 virus ...............................35

2.3.3 Cell type specific H5N1 virus infection...........................................35

2.3.4 Retrograde transport of H5N1 virus in vitro...................................35

2.3.5 Increased phosphorylation of alpha-synuclein protein in infected

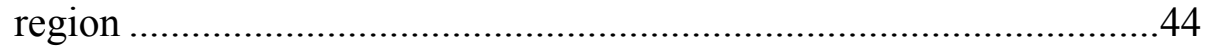

2.3.6 Apoptosis and long-term activation of microglia ...........................44

2.3.7 A reduction in the number of tyrosine hydroxylase $(\mathrm{TH})$ positive dopaminergic neuron in the SNpc.................................................44

2.4 Discussion

\section{CHAPTER 3. INFLAMMATORY EFFECTS OF HIGHLY PATHOGENIC H5N1 INFLUENZA VIRAL INFECTION IN THE CENTRAL

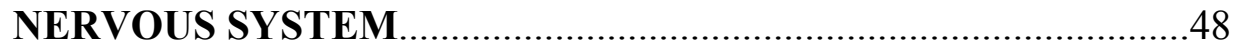

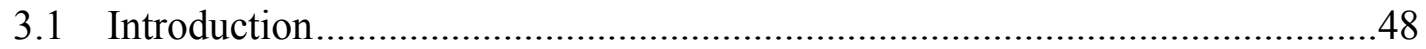

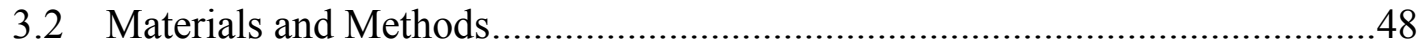

3.2.1 Virus stock preparation and inoculation of mice with $\mathrm{H} 5 \mathrm{~N} 1$..............49

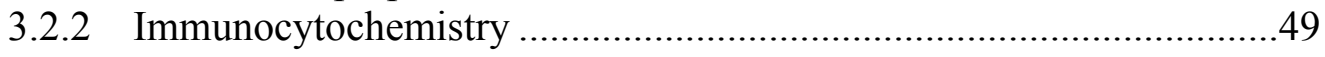

3.2.3 Stereological cell count using the optical fractionator methods .........49

3.2.4 Biochemical measurement of monoamine neurotransmitters.............50

3.2.5 Quantification of cytokines, chemokines and growth factors..............50

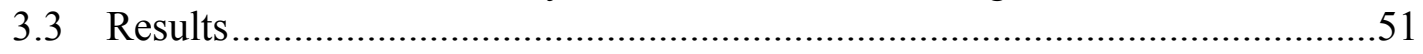

3.3.1 Transient loss of TH-positive phenotype in dopaminergic neurons following $\mathrm{H} 5 \mathrm{~N} 1$ infection .51

3.3.2 Transient reduction of dopamine in striatum following $\mathrm{H} 5 \mathrm{~N} 1$ infection. 
3.3.3 Effect of H5N1 infection on NE and 5-HT levels in the CNS. .54

3.3.4 H5N1 infection increases the number of microglia in the $\mathrm{SNpc}$.........54

3.3.5 Effect of H5N1 infection on levels of cytokines, chemokines

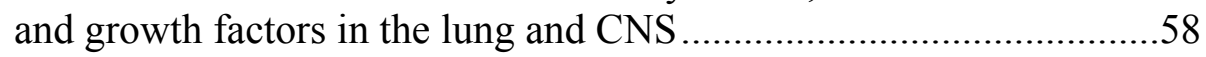

3.4 Discussion

\section{CHAPTER 4. PRIOR H5N1 INFLUENZA VIRUS INFECTION CONFERS PROTECTION FROM PARAQUAT-INDUCED

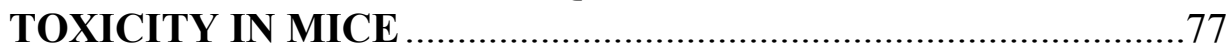

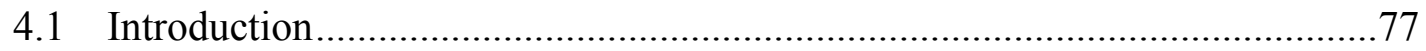

4.2 Materials and Methods...............................................................................

4.2.1 Virus stock preparation and inoculation of mice with H5N1 ..............77

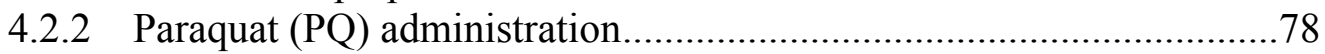

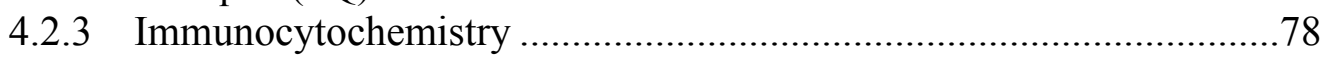

4.2.4 Stereological cell count using the optical fractionator method.............78

4.2.5 Biochemical measurement of monoamine neurotransmitters...............79

4.2.6 Quantification of cytokines, chemokines and growth factors..............79

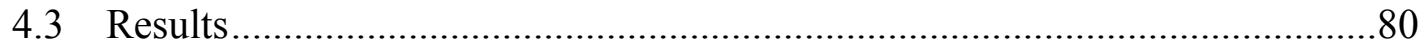

4.3.1 Does H5N1 infection synergize with PQ to alter dopaminergic neuron number in the SNpc?............................................................8

4.3.2 Does prior infection with H5N1 synergize with PQ to effect changes in dopamine, DOPAC and HVA levels in the CNS? ..............80

4.3.3 Does prior infection with $\mathrm{H} 5 \mathrm{~N} 1$ synergize with $\mathrm{PQ}$ to effect changes in NE, 5-HT and 5-HIAA levels in the CNS?.......................82

4.3.4 Effect of PQ and H5N1 on activation of the immune

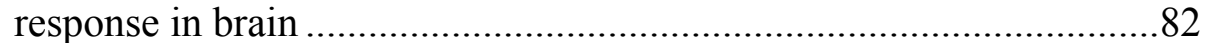

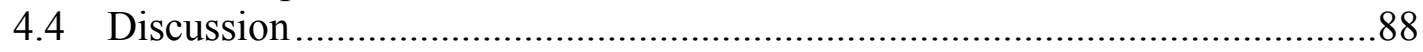

CHAPTER 5. IMPLICATIONS, LIMITATIONS AND FUTURE STUDIES …...106

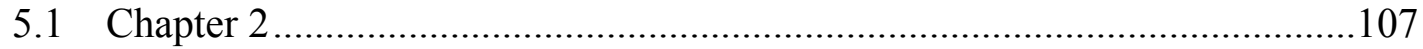

5.1.1 Implications and limitations...........................................................107

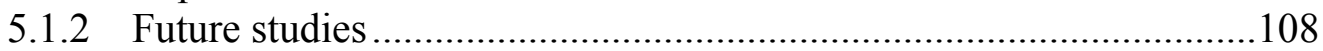

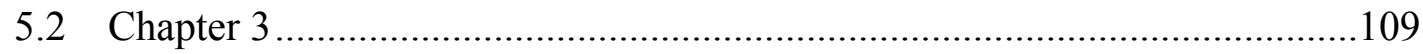

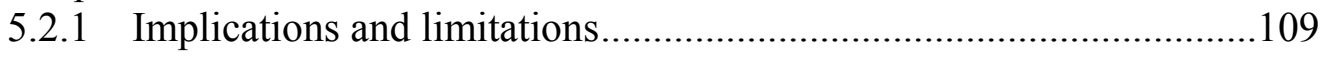

5.2.2 Future studies ........................................................................... 110

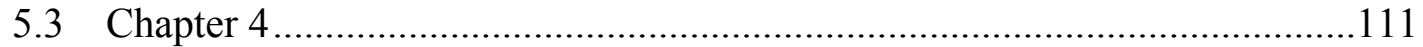

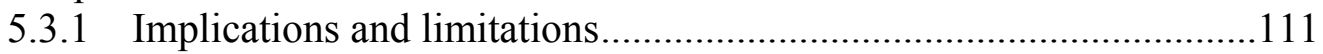

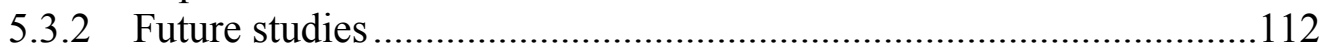

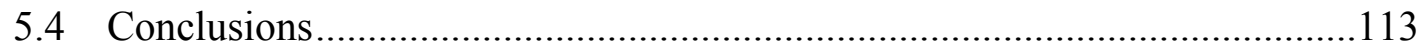

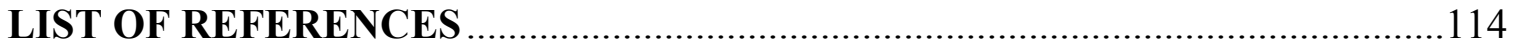

VITA. 


\section{LIST OF TABLES}

Table 1.1 Locations and Types of Mutations of the Familial PD-Related Genes .......5

Table 1.2 Association of Virus and Parkinsonism ...........................................

Table 2.1 Semi-Quantitative Analysis of H5N1 Expression in the CNS and Lung ..36

Table 2.2 Relative Density Measurements of pSer129SYN Expression in Brain.....46

Table 3.1 Level of Cytokines, Chemokines and Growth Factors in the Lung Following H5N1 Infection....

Table 3.2 Level of Cytokines, Chemokines and Growth Factors in the Brainstem

Following H5N1 Infection

Table 3.3 Level of Cytokines, Chemokines and Growth Factors in the SN

Following H5N1 Infection

Table 3.4 Level of Cytokines, Chemokines and Growth Factors in the Striatum

Following H5N1 Infection.

Table 3.5 Level of Cytokines, Chemokines and Growth Factors in the Cortex

Following H5N1 Infection

Table 4.1 Level of Cytokines, Chemokines and Growth Factors in the Lung Following H5N1 Infection and PQ Treatment

Table 4.2 Level of Cytokines, Chemokines and Growth Factors in the SN

Following H5N1 Infection and PQ Treatment.

Table 4.3 Level of Cytokines, Chemokines and Growth Factors in the Striatum

Following H5N1 Infection and PQ Treatment

Table 4.4 Level of Cytokines, Chemokines and Growth Factors in the Brainstem| Following H5N1 Infection and PQ Treatment.

Table 4.5 Level of Cytokines, Chemokines and Growth Factors in the Cortex Following H5N1 Infection and PQ Treatment. 


\section{LIST OF FIGURES}

Figure 1.1 The Functional Neuroanatomy of the Basal Ganglia................................

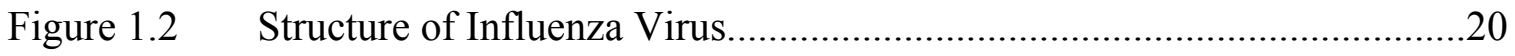

Figure 1.3 Life Cycle of Influenza Virus ...........................................................23

Figure 2.1 Percent Survival and Body Weight Change in the Infected Animals ........34

Figure 2.2 Localization of H5N1 Influenza Virus in the Nervous System ................37

Figure 2.3 Localization of H5N1 Influenza Virus in Lung, Enteric Nervous

System, Olfactory Bulb and Brainstem ..............................................39

Figure 2.4 Localization of H5N1 Influenza Virus in Brainstem, Diencephalon and Cortex ...............................................................................4 41

Figure 2.5 Transport of H5N1 Virus through Axons in Microfluidic Chambers........43

Figure 2.6 H5N1 Influenza Virus Induces a Parkinsonian Phenotype.......................45

Figure 3.1 The Size and Number of TH-Positive Dopaminergic Neurons .................52

Figure 3.2 Percent Change in the Amount of DA, DOPAC, and HVA in Brain Following H5N1 Infection..............................................................53

Figure 3.3 Percent Change in the Amount of NE in Brain Following H5N1

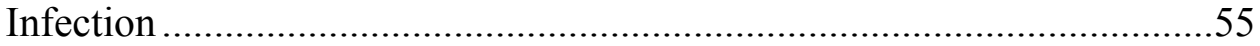

Figure 3.4 Percent Change in the Amount of 5-HT and 5-HIAA in Brain Following H5N1 Infection...............................................................56

Figure 3.5 H5N1 Infection Increased the Number of Activated Microglia ................57

Figure 3.6 Four Distinct Temporal Patterns Seen in Cytokines, Chemokines and Growth Factors Expression Following H5N1 Infection .....

Figure 3.7 Expression of Cytokines, Chemokines and Growth Factors in the Lung Following H5N1 Infection

Figure 3.8 Expression of Cytokines, Chemokines and Growth Factors in the Brainstem Following H5N1 Infection 
Figure 3.9 Expression of Cytokines, Chemokines and Growth Factors in the SN Following H5N1 Infection

Figure 3.10 Expression of Cytokines, Chemokines and Growth Factors in the Striatum Following H5N1 Infection

Figure 3.11 Expression of Cytokines, Chemokines and Growth Factors in the Cortex Following H5N1 Infection

Figure 4.1 Change in the Number of TH-Positive Dopaminergic Neurons

Following H5N1 Infection and PQ Treatment.

Figure 4.2 Percent Change in the Monoamine Neurotransmitter Level in the Striatum Following H5N1 Infection and PQ Treatment

Figure 4.3 Percent Change in the Monoamine Neurotransmitter Level in the SN Following H5N1 Infection and PQ Treatment

Figure 4.4 Percent Change in the Monoamine Neurotransmitter Level in the Brainstem Following H5N1 Infection and PQ Treatment

Figure 4.5 Percent Change in the Monoamine Neurotransmitter Level in the Hippocampus Following H5N1 Infection and PQ Treatment .........86

Figure 4.6 Percent Change in the Monoamine Neurotransmitter Level in the Cortex Following H5N1 Infection and PQ Treatment.

Figure 4.7 Expression of Cytokines, Chemokines and Growth Factors in the Lung Following H5N1 Infection and PQ Treatment.

Figure 4.8 Expression of Cytokines, Chemokines and Growth Factors in the SN Following H5N1 Infection and PQ Treatment

Figure 4.9 Expression of Cytokines, Chemokines and Growth Factors in the Striatum Following H5N1 Infection and PQ Treatment .95

Figure 4.10 Expression of Cytokines, Chemokines and Growth Factors in the Brainstem Following H5N1 Infection and PQ Treatment

Figure 4.11 Expression of Cytokines, Chemokines and Growth Factors in the Cortex Following H5N1 Infection and PQ Treatment. 101 


\section{LIST OF ABBREVIATIONS}

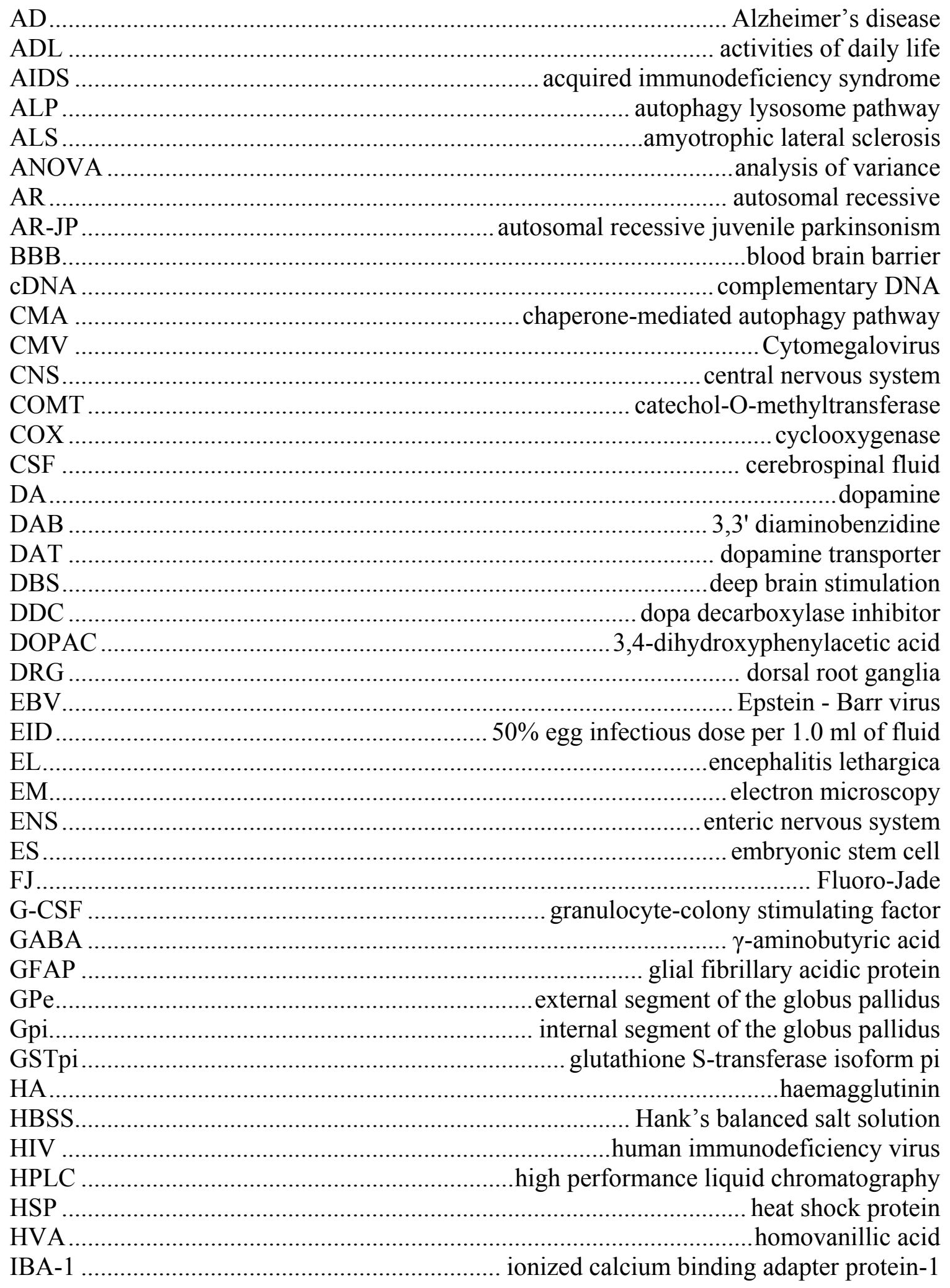


IP interferon-inducible protein

JEBV Japanese encephalitis B JNK .c-Jun N-terminal kinases

LB .Lewy Body

L-DOPA levodopa

LN

LPS

LRRK2

MAO

$\mathrm{MCP}$

MHC

MIP

$\mathrm{MPP}+$

MPPP

MPTP

NA

NE

NP

NS

NSAID

$\mathrm{M}$

MSN

NAC

nNOS

$\mathrm{NO}$

$\mathrm{O} 2$

$\mathrm{OH}$

ONOO

PA

PB

$\mathrm{PD}$

PEP

PET

PFU

PINK-1

PM

PPL-1

PPM1/2

RNP

ROS

SA

SN

SNCA
Lewy neurites lipopolysaccharide leucine-rich repeat kinase 2 monoamine oxidase monocyte chemotactic protein major histocompatibility complex macrophage inflammatory protein 1-methyl-4-phenylpyridinium 1-methyl-4-phenyl-4-propionoxypiperidine 1-methyl-4-phenyl-1,2,3,6-tetrahydropyridine neuraminidase norepinephrine nucleoprotein non-structural protein non-steroid-anti-inflammation-drug matrix protein medium spiny neurons non-amyloid component neuronal nitric oxide synthase nitric oxide superoxide anion hydroxyl peroxynitrite anion acidic polymerase protein basic polymerase Parkinson's disease postencephalic parkinsonism positron emission tomography plaque-forming unit .PTEN induced putative kinase 1 point mutation protocerebral posterior lateral cluster protocerebral posterior medial cluster ribonucleoprotein ..reactive oxygen species sialic acid substantia nigra alpha-synuclein 


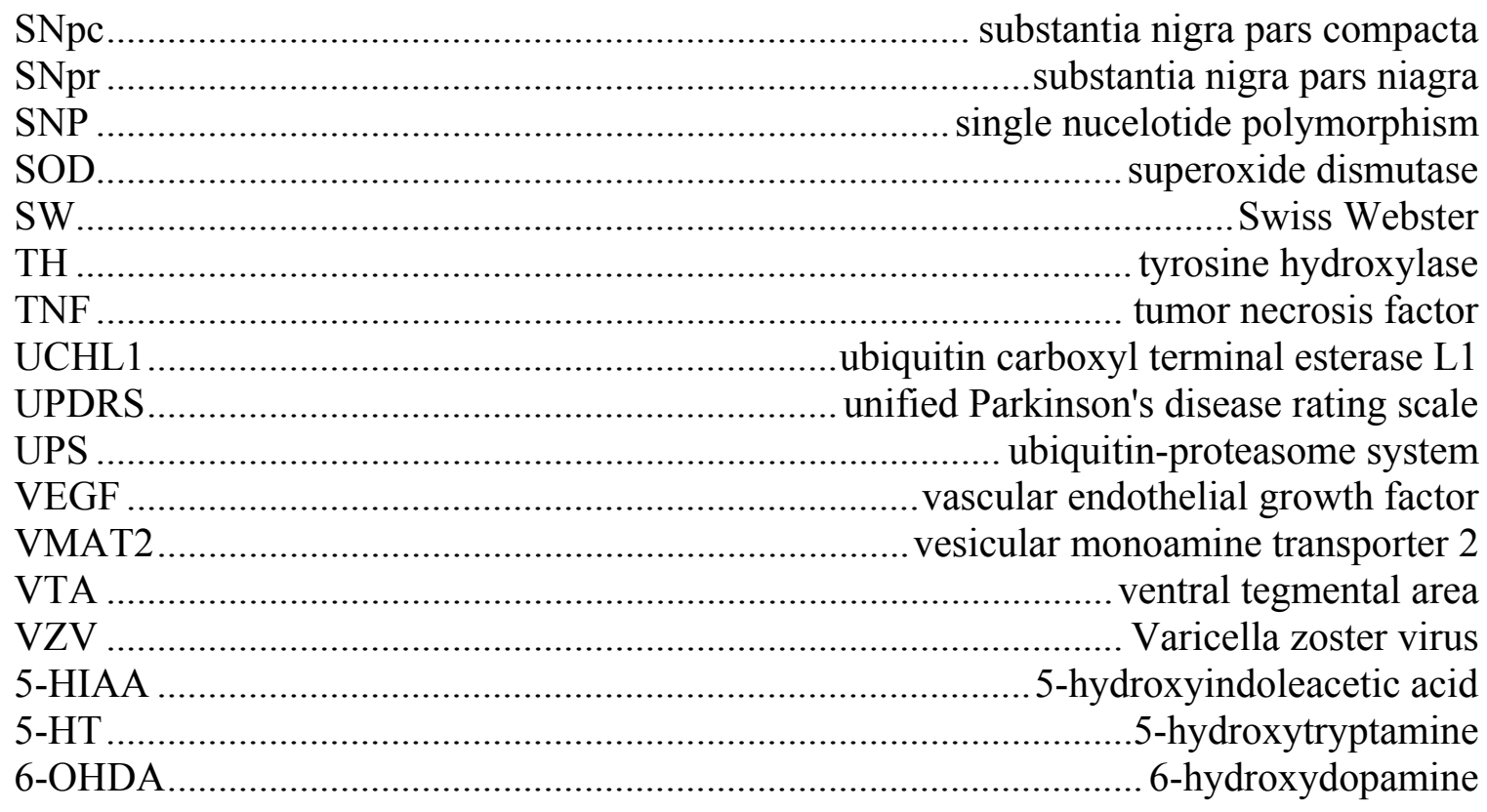




\section{CHAPTER 1. INTRODUCTION*}

1.1 Parkinson's Disease (PD)

Parkinson's disease (PD) was first described in 1817 by Dr. James Parkinson in a paper entitled "An Essay on the Shaking Palsy" (1). PD is a progressive neurodegenerative disorder that is characterized by both motor and autonomic symptoms. The most common motor symptoms include bradykinesia, hypokinesia/akinesia, rigidity, tremor and postural imbalance. The incidence of PD is approximately $1-2 \%$ of the adult population older than 50 years of age. Current estimates from the Parkinson's Disease Foundation put the number of people suffering from this disease at 4.1 million worldwide. It is predicted to rise to 8.7 million based on a projected increase in lifespan. In fact, there are more individuals diagnosed with PD than with multiple sclerosis, muscular dystrophy and amyotrophic lateral sclerosis (Lou Gehrig's Disease) combined (2).

\subsection{Functional Neuroanatomy Underlying PD}

\subsubsection{Basal ganglia}

The basal ganglion is made up of a group of nuclei located at the base of the forebrain and midbrain that sends projections to motor cortex, thalamus and mesencephalic structures. In humans, the basal ganglia is comprised of the striatum, the pallidum that includes the internal and external segment of globus pallidus, the substantia nigra, and the subthalamic nucleus (3).

\subsubsection{The circuitry of basal ganglia}

The basal ganglia receive external information from most of the cerebral cortex, thalamic nuclei, and limbic structures. The striatum, which is made of two distinct parts, the caudate and the putamen, is the main input structure of the basal ganglia. It sends its output signal to other nuclei of the basal ganglia, including the pallidum and the substantia nigra pars reticulata. Within the striatum, the majority of synapses occur on GABA( $\gamma$-aminobutyric acid)ergic, medium spiny neurons (MSN), that make up about $95 \sim 97 \%$ of the total population of striatal neurons (4). The internal segment of the globus pallidus (GPi) and the substantia nigra pars reticulata (SNpr) provide the main output from the basal ganglia.

* Sections 1.4 and 1.8 of this chapter adapted with permission. Jang H, Boltz DA, Webster RG, Smeyne RJ. Viral parkinsonism. Biochim Biophys Acta 1792(7), 714-721, (2009) (5). 
The GPi receives input signals from the striatum directly or indirectly via the subthalamic nucleus (STN) followed by the external segment of the globus pallidus (GPe).The GPi sends projections to the thalamic nuclei that innervate the motor-related areas of the cortex. The pallidal neurons are tonically active in the absence of an input signal and stop firing when they receive inhibitory input from the striatum. The pallidal neurons are inhibitory GABAergic neurons, as a result, the input from the striatum removes the tonic inhibition from the target of the pallidal neurons (4). The input and the output components form two closed circuits that begin and end at the cerebral cortex (Fig 1.1A). In the direct pathway, signal is relayed the following order, cerebral cortex $\rightarrow$ striatum (inhibitory) $\rightarrow$ GPi (inhibitory) $\rightarrow$ thalamus $\rightarrow$ cerebral cortex, so that the net effect on the cortex is excitatory. In the direct pathway, dopaminergic neurons of the SN synapse on D1 receptors in the striatum, providing a dopaminergic stimulatory response to the striatal projecting neurons. In the indirect pathway, the components communicate in the following order, cerebral cortex $\rightarrow$ striatum (inhibitory) $\rightarrow$ GPe (inhibitory) $\rightarrow$ STN (excitatory) $\rightarrow$ GPi (inhibitory) $\rightarrow$ thalamus $\rightarrow$ cerebral cortex, so that the net effect on the cortex is inhibitory. In contrast to the direct pathway, dopamine acts on the striatal projection neurons through the dopamine D2 receptor which is inhibitory in response to dopamine stimulation (4).

\subsubsection{Basal ganglia and PD}

The major motor symptoms of PD appear when approximately $60 \%$ of the dopaminergic neurons have degenerated. The subsequent reduction in striatal dopamine affects the two pathways differentially. Low DA input to the stimulatory D1 receptors results in less firing of inhibitory striatal neurons in the direct pathway. As a result, neurons in GPi become more active and inhibit the thalamic neurons. The reduction of dopamine in the indirect pathway provides less activation of $\mathrm{D} 2$ receptors and consequently, less inhibition of the striatal neurons. Consequently, inhibitory striatal neurons become more active and increase the inhibition of neurons in the GPe.

Sequentially, excitatory STN neurons increase the excitatory input to the neurons in the $\mathrm{GPi}$, enhancing the firing of GPi, and further suppressing the activity of thalamic neurons. Overall, low DA input gives rise to a stronger inhibitory tone of basal ganglia output to their targets through both the direct and indirect pathways. This is the cause of the bradykinesia and the hypokinesia symptoms observed in PD (Fig 1.1B) (3).

\subsection{Epidemiology of PD}

The estimated prevalence of PD in industrialized countries is $0.3 \%$ of the total population and $1 \%$ in people over 60 years of age. Regionally, North America, South America, and Europe record the highest prevalence of PD, while Asia and Africa record the lowest prevalence. Because the prevalence rates are affected by the difference in the survival of the patients, the incidence rates are a more reliable indicator of PD risk. Recently reported annual incidence rates, having been adjusted for age and/or gender, are 13.4 per 100,000 people per year in industrialized countries (2). 

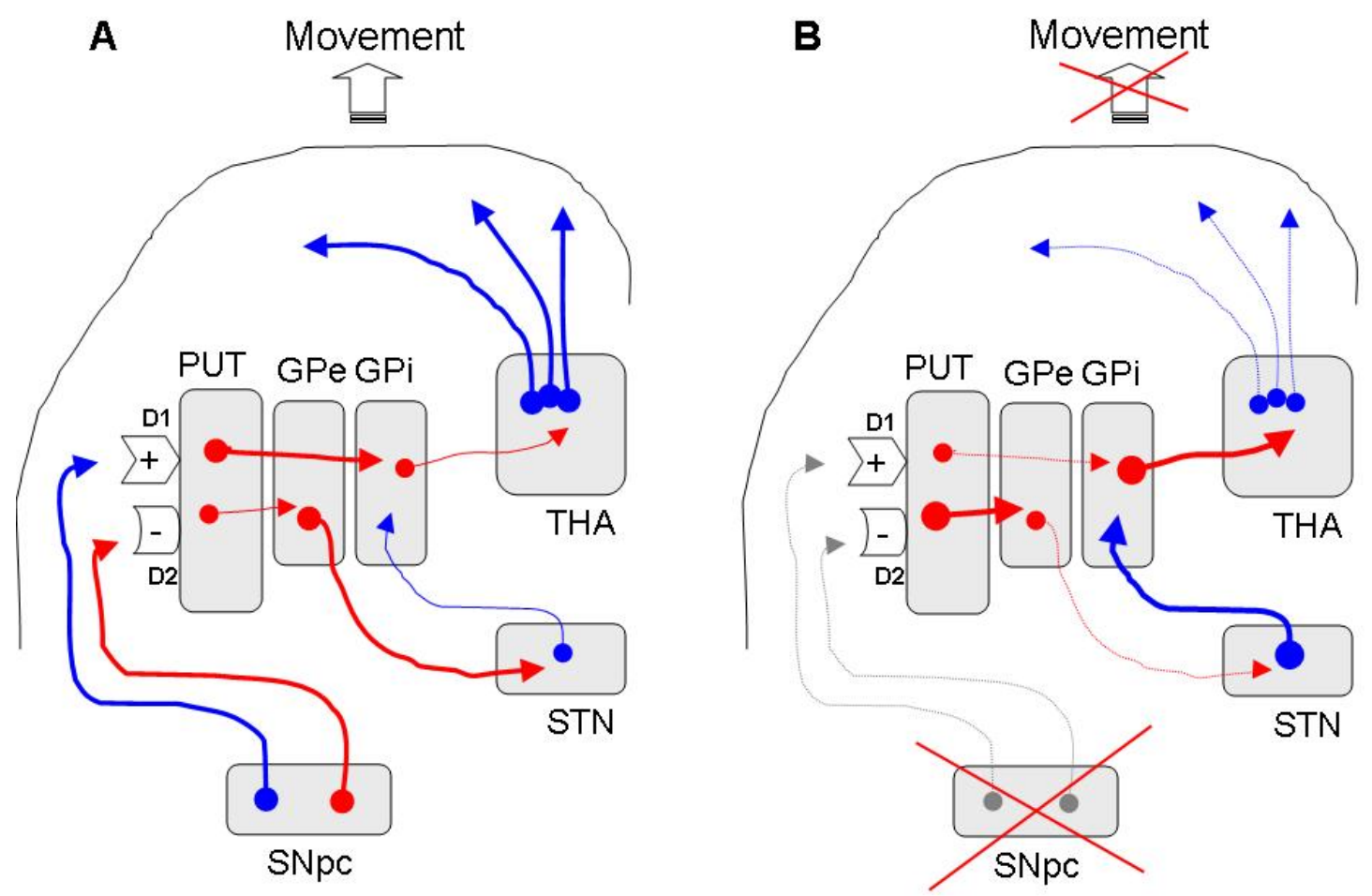

Figure 1.1 The Functional Neuroanatomy of the Basal Ganglia

These diagrams demonstrate the circuitry of the basal ganglia in normal (A) and PD brain (B). The output of the basal ganglia is regulated by the fine balance between the direct and indirect pathways that both promote and suppress body movements, respectively. In the normal state, the afferent dopaminergic signal from the substantia nigra pars compacta (SNpc) enhances the direct pathway and suppresses the indirect pathway, producing a positive net effect on the stimulation of cortical motor areas. In PD, the depletion of dopamine in the striatum gives rise to an imbalance and suppression of the thalamocortical activity. Blue lines represent the excitatory pathways and red lines display the inhibitory pathway. GP, globus pallidus; GPe, external segment of GP; GPi, internal segment of GP; STN, subthalamus nucleus; D1, D1 dopamine receptors; D2, D2 dopamine receptors.

Source: Modified with permission from Elsevier. Albin RL, Young AB, and Penney, JB. The functional anatomy of basal ganglia disorders. Trends Neuroscience 12, 366-375 (1989) (6). 
Age is the most potent risk factor that contributes to the incidence of developing Parkinson's disease. The incidence rates increase rapidly over 60 years of age, with only $4 \%$ of PD patients developing the disease when they are under the age of 50 years (2). Gender is also a factor that contributes to PD incidence rates. For men, the incidence rate is 19 per 100,000 , which are $91 \%$ higher than that of women, whose incidence is 9.9 per 100,000 . Based on race/ethnicity, the highest incidence rates are recorded for Hispanic populations (16.6), followed by non-Hispanic white (13.6), Asian (11.3), and Black (10.2 per 100,000$)$ populations, suggesting that race/ethnicity also affects the incidence of $\mathrm{PD}(2)$.

\subsection{Etiology of PD}

The underlying cause(s) for the vast majority of PD cases are unknown. Controversy still exists as to how much of the disease results from strict genetic causation, a purely environmental factor, or the combination of the two risk factors $(7,8)$. Empirical evidence suggests that less than $10 \%$ of all diagnosed parkinsonism has a strict familial etiology. At this time, there have been 13 identified parkinsonian loci, although mutations in just five of these genes, alpha-synuclein (PARK 1), parkin (PARK2), PINK1 (PARK6), DJ-1 (PARK7), and LRRK2 (PARK8) make up the bulk of these cases (9). In addition to genetic mutations leading to the onset of Parkinson's disease, there are also clear environmental factors that lead to parkinsonism. These cases of parkinsonism are often referred to as secondary parkinsonism and can result from a myriad of causes including infectious agents (10), drugs (11), toxins (12), vascular insults (13), trauma (14), and although rare, other pathological problems such as tumors $(15,16)$.

\subsubsection{Genetic factors}

At this time, there have been 13 identified parkinsonian loci, although there are mutations in just eight of these genes, alpha-synuclein (PARK 1), parkin (PARK2), UCH-L1 (PARK5), PINK1 (PARK6), DJ-1 (PARK7), LRRK2 (PARK8), ATP13A2 (PARK9), and Omi/HtrA2 (PARK13) (Table 1.1). However, these cases of familial PD account for less than $10 \%$ of the total number of PD cases (9).

\subsubsection{Environmental toxin factors}

The role of environmental toxins has been a focus of PD research in recent years in response to the "Case of the Frozen Addicts". People attempting to synthesize a designer narcotic drug accidentally produced 1-methyl-4-phenyl-1,2,3,6tetrahydropyridine (MPTP) that when injected, inflicted the user with a syndrome almost identical to idiopathic PD. Later, MPTP was shown to be a neurotoxin which selectively kills dopaminergic cells in the SNpc in both humans and non-human primates (17). Once administered, MPTP crosses the blood-brain barrier and is converted to its toxic form, 
Table 1.1 Locations and Types of Mutations of the Familial PD-Related Genes

\begin{tabular}{lllll}
\hline Locus & Gene & $\begin{array}{l}\text { Chromosomal } \\
\text { location }\end{array}$ & Type of mutation & Inheritance \\
\hline PARK1 & SNCA & $4 \mathrm{q}$ & PM, Dupl, Tripl & AD \\
PARK2 & Parkin & $6 \mathrm{q}$ & $\begin{array}{l}\text { PM, Dupl, Tripl, Ins, } \\
\text { Del }\end{array}$ & AR \\
PARK5 & UCH-L1 & $4 \mathrm{p}$ & PM & AD \\
PARK6 & PINK1 & $1 \mathrm{p}$ & PM, Ins, Del & AR \\
PARK7 & DJ-1 & $1 \mathrm{p}$ & PM, Del & AR \\
PARK8 & LRRK2 & $12 \mathrm{p}-\mathrm{q}$ & PM, Del & AD \\
PARK9 & ATP13A2 & $1 \mathrm{p}$ & PM, Del & AR \\
PARK13 & Omi/HtrA2 & $2 \mathrm{p}$ & PM & AD \\
\hline
\end{tabular}

SNCA, alpha-synuclein; UCH-L1, Ubiquitin carboxyl-terminal hydrolase L1; PINK-1, PTEN induced putative kinase 1; LRRK2, leucine-rich repeat kinase 2; PM, point mutation; Ins, insertion; Del, deletion; Dupl, duplication; Tripl, triplication; AR, autosomal recessive; $\mathrm{AD}$, autosomal dominant.

Source: Modified with permission from Blackwell Publishing. Carola Schiesling, Nicole Kieper, Kay Seidel and Rejko Krüger. Review: Familial Parkinson's disease - genetics, clinical phenotype and neuropathology in relation to the common sporadic form of the disease. Neuropathology and Applied Neurobiology 34, 255-271(2008)(18). 
1-methyl-4-phenyl-pyridinium (MPP+) by monoamine oxidase $\mathrm{B}(\mathrm{MAO}-\mathrm{B})$ in glial cells (19). MPP+ is taken up by dopaminergic neurons through the dopamine transporter (DAT). In neurons, MPP+ inhibits complex I of mitochondria and induces oxidative stress (12).

Paraquat is a widely used herbicide throughout the world. Investigators have become interested in paraquat, because in addition to sharing structural similarities to MPP+, which is a toxic form of MPTP, it also showed an etiological link to PD in epidemiological studies. Paraquat is reduced by several reductases, including mitochondrial complex I, to become a PQ monocation free radical $(\mathrm{PQ}+)$. Subsequently, the PQ free radical is rapidly oxidized by oxygen and produces superoxide radicals and reactive oxygen species that can be toxic to the cell (20).

Rotenone was first isolated from the roots and stems of several plants and has been used as a pesticide and fish poison $(21,22)$. Highly lipophilic, rotenone easily penetrates the blood-brain barrier and accumulates in the mitochondria, inhibiting complex I of the electron transport chain.

Epidemiological studies have demonstrated that the residents of rural areas where herbicides and pesticides were used extensively, have higher incidences of PD than the residents of urban areas (23). However, further research is needed to implicate any specific toxin as a cause of sporadic PD.

\subsubsection{Viral factors}

A number of viruses, including influenza, coxsackie, Japanese encephalitis B, western equine encephalitis, herpes, and human immunodeficiency virus (HIV), have been associated with both acute and chronic parkinsonism (Table 1.2).

The hypothesis that viruses or other contaminating agents may be a factor initiating primary or secondary parkinsonism often relates to coincidental cases of parkinsonism that lie outside of the expected population (5). One of the most famous and still controversial examples is the parkinsonism that occurred subsequent to a viral encephalopathy that developed following the 1918 influenza pandemic (24). Another example that suggests viral agents can act as an initiator of parkinsonism is the appearance of parkinsonian clusters. These are groups of individuals who share common environments and develop parkinsonism at greater than normal statistical rates without the typical risk factors. In fact, the risk of developing Parkinson's disease is approximately 2 times greater for people sharing close quarters, including doctors and nurses, teachers, and religion-related jobs. Several of these parkinsonian clusters have been described, including those living in Israeli kibbutz's, a group of college teachers, garment workers in a manufacturing factory, a group of actors and producers and technical staff working on a television series in Canada $(25,26)$. 
Table 1.2 Association of Virus and Parkinsonism

\begin{tabular}{|c|c|c|c|}
\hline Virus & Family & Species & References \\
\hline \multirow{4}{*}{ DNA } & \multirow{4}{*}{ Herpesviridae } & Herpes simplex virus & $(27-30)$ \\
\hline & & Epstein-Barr virus & $(31)$ \\
\hline & & Cytomegalovirus (CMV) & $(28,30)$ \\
\hline & & Varicella zoster virus (VZV) & $(32)$ \\
\hline \multirow{10}{*}{ RNA } & Bornaviridae & Borna disease virus & $(33)$ \\
\hline & Orthomyxoviridae & Influenza virus Type A & $(34-43)$ \\
\hline & Paramyxoviridae & Measles & $(44,45)$ \\
\hline & \multirow{3}{*}{ Picornaviridae } & Coxsackie virus & $(46-49)$ \\
\hline & & Echo virus & $(50)$ \\
\hline & & Polio virus & $(51)$ \\
\hline & \multirow[t]{2}{*}{ Retroviridae } & Human immunodeficiency virus (HIV) & $(52-57)$ \\
\hline & & West Nile virus & $(58)$ \\
\hline & \multirow[t]{2}{*}{ Flaviviridae } & Japanese encephalitis B virus & $(34,59-66)$ \\
\hline & & St. Louis virus & $(67-69)$ \\
\hline
\end{tabular}

Source: Modified with permission from Elsevier. Haeman Jang, David A. Boltz, Robert G. Webster, Richard Jay Smeyne. Viral parkinsonism. Biochimica et Biophysica Acta (BBA) 1792(7), 714-721 (2009)(5). 


\subsubsection{Combination of multiple factors}

It is hypothesized that PD is caused not by a single event but by a combination of multiple hit throughout the life of the individual (70-73). If PD results from a combination of various insults (including genetic defects, environmental toxin exposure, viral infection, natural loss of DA neurons, and/or normal aging), the event that initiates disease progression may occur far earlier than the onset of symptoms. Indeed, McCormack et al., showed that the exposure to an environmental toxin such as PQ during the early postnatal period made the SN more vulnerable to the neurotoxin in adult mice (74). In addition, rodents that have been exposed to the bacterial lipopolysaccharide (LPS) during prenatal stages show decreased resistance to 6-hydroxydopamine (6OHDA) during their adult period (75). These results suggest that these insults in early life increase susceptibility to the effects of neurotoxins later in life.

\subsection{Pathology of PD}

The main neuropathological defect observed in PD is the selective loss of the pigmented cells located in the substantia nigra pars compacta (SNpc). Cell loss has also been described in the locus coeruleus (76), the dorsal motor nucleus of the vagus nerve (77) and throughout the autonomic nervous system (78). The pigmented cells in the SNpc are dopamine (DA) producing neurons, and consequently the loss of these cells results in a reduction in the number of both afferent fibers and the amount of DA to its main target, the striatum (79). When patients lose approximately $70 \sim 80 \%$ of their striatal DA and $50 \sim 60 \%$ of the dopaminergic neurons in the substantia nigra pars compacta (SNpc), the typical constellation of parkinsonian symptoms become evident (80). Because the progression of cell loss is thought to occur over a protracted period of time in a defined spatiotemporal manner, the onset of Parkinson's disease symptoms are often insidious $(81,82)$.

In addition to neuronal loss, primary $\mathrm{PD}$ is also defined by the presence of intracytoplasmic aggregates, Lewy bodies (LB) and intraprocess aggregates, Lewy neurites (LN). The presence of LB is not limited to the SNpc, but distributed in the olfactory bulb, hypothalamus, locus ceruleus, cerebral cortex, vagus nerve, and myenteric plexus of the autonomic nervous system (83). LB were first described and linked to PD by Frederic Lewy (84) and subsequent studies using electron microscopy revealed that these inclusions are made of an unknown filament (85).

\subsubsection{The role of Lewy bodies $(L B)$}

It is debated if the abnormal protein aggregation observed in various neurodegenerative diseases is protective or deleterious to the cell. The role of LB in PD is also unclear. It has been suggested that LB containing-dopaminergic neurons in the SNpc are less vulnerable to cell death than LB negative dopaminergic neurons (86). In this view, LB can be thought of as resulting from a cellular defensive mechanism like the 
formation of an aggresome (87). In the cell, the appearance of misfolded or abnormal proteins are managed by several cellular protective mechanisms such as the molecular chaperones, ubiquitin-proteasome system (UPS) and autophagy lysosome pathway (ALP) (87). When the amount of abnormal or aggregated protein exceed the capacity of the UPS and/or ALP, cells can sequester them at a single cytoplasmic site near the centriole and generate a large inclusion body called an aggresome(88). The aggresome recruits UPS components and may facilitate abnormal protein degradation within itself. After formation, the aggresome may persist in an insoluble form or be cleared by an unknown mechanism (89).

Like the aggresome, LB contain many components of the UPS including ubiquitin-activating enzyme (E1), ubiquitin-conjugating enzyme (E2), ubiquitin-ligase (E3), proteasome activator, and heat shock protein(90-92). LB also contain specific aggresomal proteins such as $\gamma$-tubulin and pericentrin. In a cell culture model, the proteolytic stresses driven by proteasome inhibitors or by the over-expression of protein induce the formation of aggresome-like inclusions in dopaminergic cells. These inclusions have a dense core and peripheral halo, mimicking the shape of LB (93). These evidences suggest that LB may be formed by the cellular protective mechanism.

\subsubsection{The structure and function of $\alpha$-synuclein}

In 1997, Spillantini et al, were first to describe $\alpha$-synuclein protein as the main component of LB and LN in idiopathic PD (94). At a similar time, a missense mutation in the $\alpha$-synuclein gene was reported in familial PD by another group (95). $\alpha$-synuclein is a member of the synuclein family that includes $\beta$-synuclein and $\gamma$-synuclein. $\alpha$-synuclein consists of 140-amino acid residues and has three distinct domains. The amino-terminal domain (residues 1-70) carries incomplete KTKEGV repeats (96). The hydrophobic domain (residues 71-82), also called the non-amyloid component (NAC), appears to be accountable for the aggregation of $\alpha$-synuclein (97). The carboxyl-terminal domain (residues 96-140) consists of acidic residues and contains three highly conserved tyrosine residues. This region appears to be involved in the solubilization of high molecular weight proteins and may regulate the aggregation of full length $\alpha$-synuclein (98). Also, the deletion of this domain results in the loss of chaperone activity of $\alpha$-synuclein (99).

The function of $\alpha$-synuclein still requires clarification. It is known that $\alpha-$ synuclein binds to the lipid membrane and selectively inhibits phospholipase D2, which may be involved in cytoskeletal regulation and endocytosis. Synphilin-1 is also known to bind to $\alpha$-synuclein where it may act as an adaptor molecule, since it contains ankyrin-

like repeats, a coiled-coil domain and an ATP/GTP binding domain. In vitro, synphilin is co-localized with $\alpha$-synuclein in neurons and facilitates the formation of cytosolic phasedense inclusions (100). In vivo, synphilin-1 is detected in the Lewy bodies of idiopathic PD (101). These observations suggest that Synphilin-1 may modulate the aggregation of $\alpha$-synuclein and the formation of Lewy bodies. 
$\alpha$-synuclein knock-out mice show morphologically intact brain architecture and have no structural deficit in dopaminergic cell bodies, processes and synapses. In addition, these mice display normal long term potentiation (LTP) in the hippocampus (102) and no deficit in spatial learning (103). However, they show enhanced DA release with coupled stimuli by using $\mathrm{Ca}^{2+}$, and decreased striatal DA and DA dependent locomotion with amphetamine administration. Based on these observation, Abeliovich et al., suggest that $\alpha$-synuclein may act as an "activity-dependent negative regulator of DA neurotransmission" in the presynaptic terminal (102).

\subsubsection{Association of $\alpha$-synuclein with the formation of $L B$ in $P D$}

In mammals, $\alpha$-synuclein is predominately expressed in the presynaptic nerve terminals of the olfactory bulb, amygdala, hippocampus, and cerebral cortex (104). The post-translational modifications of $\alpha$-synuclein are not fully understood. However, in $\alpha-$ synuclein transfected cells, it is frequently phosphorylated at serine 129 (105). In addition, it has been discovered that phosphorylation at serine 129 produces dominant pathological changes in familial and idiopathic PD (106).

The mechanism by which soluble monomeric $\alpha$-synuclein leaves its assumed binding site in presynaptic terminals, combines with other LB components such as ubiquitin, and forms insoluble LB and LN (107) is largely unknown. Emerging evidence demonstrates that multiple factors may be involved in the aggregation of $\alpha$-synuclein and the formation of LB in PD (108).

Mutations of the $\alpha$-synuclein gene, including Ala53Thr, Ala30Pro, and Glu46Lys have been identified to be dominantly or recessively inherited in familial PD (95), indicating that the mutated $\alpha$-synuclein gene is directly involved in PD. Furthermore, Ala53Thr and Glu46Lys mutated $\alpha$-synuclein proteins display increased self-assembly and fibril formation in vitro (109-111). Environmental toxins, such as rotenone and paraquat, are also known to induce a conformational change in $\alpha$-synuclein and facilitate the formation of $\alpha$-synuclein fibrils in vitro (112).

A change in cellular protein degradation may also be involved in the $\alpha$-synuclein aggregation and LB formation. It has been demonstrated that $\alpha$-synuclein is a substrate for the UPS $(113,114)$ whereas the mutated $\alpha$-synuclein protein inhibits proteasomemediated degradation (114). In addition, mutations in the genes encoding two UPS enzymes, parkin (a component of a multiprotein E3 ubiquitin ligase complex) and ubiqutin C-terminal hydrolase L1 (UCH-L1), are also associated with some cases of familial PD $(115,116)$, supporting the theory that defects in the UPS may result in proteinopathies in PD.

The autophagy lysosome pathway (ALP), a catabolic process in which a cell's own components can be degraded by lysosomal machinery, also may have a role in synucleinopathies in PD. Cuervo et al., found that the wild type $\alpha$-synuclein is a substrate for lysosomal degradation and is selectively translocated into lysosomes by the 
chaperone-mediated autophagy pathway (CMA). Interestingly, the A53T and A30P $\alpha$ synuclein mutants, showing a high affinity to lysosomal membrane receptors, are targeted to lysosomes, but fail to be cleared. Rather, it inhibits the degradation of both mutant $\alpha$ synucleins and other CMA substrates (117). Thus, the accumulation of mutant synucleins as well as other CMA substrate proteins alters cellular homoeostasis and results in neurodegeneration (118).

\subsubsection{Braak's pathological observations}

Although the exact role of LB in PD is not yet clear, there is a hypothesis that the presence of LB is positively correlated with the progression of PD (119). Braak et al. assessed $\alpha$-synuclein positive LB and LN by a semi quantitative method in 41 cases of symptomatic PD, 69 subjects who might have been developing PD, but did not show parkinsonian symptoms, and 58 aged matched controls. Interestingly, the distribution of LB and LN, ranging from the brainstem to the cerebral cortex, appeared in a hierarchical manner that is correlated with PD symptomatic progression. For example, in Braak's stages 1 and 2, both of which are preclinical, the presence of LB and LN is confined to the medulla oblongata and olfactory bulb. In this stage, LB and LN initially appear on the dorsal IX/X motor nucleus (DMN) then spread into the pontine tegmentum. In Braak stages 3 and 4, LB and LN are found in the midbrain, including the SNpc, in addition to the areas affected in stage 1 and 2 . In stages 5 and 6 , in which PD symptoms have progressed to their most complete, including dementia, LB and LN are newly detected in the neocortex $(120,121)$.

These patterns of Braak's LB and LN progression are somewhat correlated with the symptoms that clinicians observe in PD patients. For example, it has been reported that autonomic symptoms, including constipation, olfactory dysfunction, and sleep disorders may precede motor symptoms by years $(122,123)$. LB and LN seen in the enteric plexuses, dorsal motor nucleus, and olfactory nucleus at Braak's stage 1 and 2 may account for the induction of these symptoms. These observations support the hypothesis that the formation of LB and LN may be initiated far earlier than when the typical motor symptoms appear, as in sporadic PD.

The question arises, what might trigger such LB pathology in the DMN, a nucleus that integrates fibers that innervate visceral organs, including the lung, heart, gastrointestinal tract as well as olfactory nucleus in the early phase of PD? Some viruses including influenza and Herpes simplex have been proposed to attack the enteric nervous system (ENS) and to be transmitted into the DMN by retrograde transport (124). Interestingly, influenza virus seems to be associated with LB formation. Interferon induced protein MxA, which plays a role in defense against influenza virus, was found in LB and swollen processes in sporadic PD (43). However, no direct evidence that influenza virus induces the formation of LB in idiopathic PD has been presented. 


\subsubsection{Neuroinflammation}

More evidence suggests that neuroinflammation may play an important role in PD progression. Two classes of glial cells, astrocytes, and microglia, are thought to play different roles in the survival of neurons. Astrocytes, the most numerous cell type in the CNS, have a role in maintaining brain homeostasis and the blood brain barrier, responding to brain damage, and producing neurotrophic factors. Microglia, the resident mononuclear phagocytic cells in the CNS, are present in a resting state but become activated following trauma or metabolic insult. They play a key role as the initial line of defense in the CNS, producing proinflammatory cytokines such as tumor necrosis factor alpha (TNF- $\alpha$ ), interleukin beta (IL-1 $\beta)$, and interferon gamma (IFN- $\gamma)(125)$.

The increased number of activated microglia and enhanced levels of proinflammatory mediators such as IL-1 $\beta$, IL-6, TNF- $\alpha$, inducible nitric oxide synthase (iNOS), and cyclooxygenase-2 (COX-2) have been detected in the cerebrospinal fluid (CSF) and the brain parenchyma of idiopathic PD patients (126-130). In addition, the postmortem study of MPTP-intoxicated PD patients reveal that gliosis and clustering of microglial cells existed decades after the initial intoxication (131). Epidemiological studies also support this notion, demonstrating that chronic non-steroid-antiinflammation-drug (NSAID) users have lower PD incidence than non regular users (132134). However, when and how neuroinflammation occurs in PD is still largely unknown.

\subsection{Treatment of PD}

\subsubsection{Pharmacological treatments}

Levodopa, a precursor molecule in DA synthesis, was first introduced for human use in the late 1960's (135) and has been the main treatment for PD for more than 40 years (136). Unlike dopamine, L-dopa can cross the blood brain barrier freely, be transported into the dopaminergic neuron through amino acid transporter systems, and be converted to dopamine by dopa-decarboxylase (137). A peripheral dopa decarboxylase inhibitor (DDC) and catechol-O-methyltransferase (COMT) are often co-administered to prolong the peripheral half life of levodopa, and increase it's availability in the CNS (138). So far, L-dopa, used in conjunction with a peripheral dopa decarboxylase inhibitor, has been shown to be the most effective way to relieve symptoms in the early and midstages of PD (139).

About $40-75 \%$ of patients who have had 4-6 years of levodopa therapy develop side effects, such as motor fluctuations and dyskinesias $(140,141)$. Fluctuations in motor performance are thought to be outcomes of the rise and fall of L-dopa levels in plasma following each daily dose (142). The progressive loss of dopaminergic neurons results in a reduction in the storage capacity of dopamine terminals. Consequently, dopamine levels in the synapse readily reflect the levodopa levels in plasma and motor fluctuations become worse (143). Dyskinesias, and choreic or dystonic involuntary movements are 
also correlated with prolonged L-dopa treatment. The exact mechanism underlying dyskinesias is not fully understood. However, it is generally accepted that the pulsatile stimulation caused by fluctuating and discontinuing levels of L-dopa in the brain may affect the output of the basal ganglia and induce dyskinesias (144)

The dopamine agonists, including bromocriptine, pergolide, pramipexole, ropinirole, lisuride, and cabergoline have been used singly or in combination with Ldopa. In contrast to L-dopa, dopamine agonists alone less provoke the motor side effects in de novo patients. However, it can cause non-motor side effects such as gastrointestinal disorders, sleep attack, and impulsive control disorder (145).

Selegiline and rasagiline, potent type B monoamine oxidase (MAO-B) inhibitors, have been used to treat PD since the 1970s (146). Although they are less effective than either L-dopa or dopamine agonists, they are well tolerated and are able to delay or reduce dopaminergic treatment by increasing the cerebral level of dopamine (147).

\subsubsection{Surgical treatments and deep brain stimulation}

Pallidotomy and thalamotomy were performed before L-dopa was developed as a drug therapy. In these procedures, small lesions are made in the globus pallidus or the ventrolateral thalamus to relieve PD related tremors or rigidity, respectively. Although such surgical procedures provided substantial improvements in PD symptoms, they often resulted in cognitive impairment and other side effects (148).

Deep brain stimulation (DBS) was introduced as a substitute to thalamic lesioning by Benabid et al. in 1987. An electrode, connected to a programmable pulse generator, is placed in, and permanently stimulates the target areas, either Gpi or STN. DBS is very effective in reducing motor fluctuation and dyskinesias in advanced PD, although it has some potential to provoke neuropsychological dysfunction (149).

\subsubsection{Human mesencephalic tissue transplantation}

The first clinical trial of transplantation of human fetal ventral mesencephalic (VM) tissue into PD patients was performed in the 1980s. VM tissues were derived from 6 -9 week old human embryos and placed on either one or both sides of the striatum (150, 151). Patients who had VM tissue implanted showed substantial improvement in the Unified Parkinson's Disease Rating Scale (UPDRS) and the activities of daily life (ADL) scale, represented by increased fluorodopa uptake on PET scan, as well as normal DA release (152). Furthermore, postmortem pathology studies at 18 months after transplantation revealed that the grafted DA neurons survived well and reinnervated the host striatum (153). Subsequently, there have been two double blind, placebo controlled

clinical trials conducted, however, these failed to recapitulate previous results. Compared to the sham control groups, the patient receiving transplants showed little or no improvement in the UPDRS, despite increased fluorodopa uptake seen in PET scanning. 
Graft-induced dyskinesias have occurred in approximately $50 \%$ of the grafted patients within 6-12 months (154).

Do dopaminergic grafts follow the host PD related pathology? Postmortem studies in four patients who died 11-16 years after surgery showed that the grafted neurons had $\alpha$-synuclein positive Lewy bodies and Lewy neurites. However, no Lewy bodies and Lewy neurites were found in grafted tissue in the patients who died 4 to 9 years after surgery. These data suggest that at least a decade is needed to develop LB pathology in the grafted tissue (155).

\subsection{Animal Models for PD}

The key features of $\mathrm{PD}$ are progressive neurodegeneration of dopaminergic neurons in the SN and motor symptoms such as tremors and bradykinesia. During the last few decades, many investigators have aimed to generate animal models that recapitulate at least some of the cardinal symptoms of PD. Two approaches, based on either genetic manipulation or neurotoxins, are being used to develop a PD model in animals.

\subsubsection{Genetic model}

\subsubsection{1 $\alpha$-synuclein}

A lot of attention has been focused on the $\alpha$-synuclein gene, because $\alpha$-synuclein is a major component of LB, and Ala53Thr, Ala30Pro, and Glu46Lys mutations have been identified as causative in dominantly or recessively inherited familial PD (95). The first transgenic mouse model that over-expressed wild type human $\alpha$-synuclein showed $\alpha$ synuclein positive neuronal inclusions in the SN, neocortex, and hippocampus. Despite the degeneration of nerve terminals in the striatum, no obvious loss of DA neurons has been found in the SN of these mice (156).

To determine the effect of $\alpha$-synuclein defects in dopaminergic neurons, wild type, A53T, and A30P $\alpha$-synuclein mutant mice were used to target $\alpha$-synuclein overexpression or mutation in cells expressing tyrosine hydroxylase (TH). Mice with a single gene mutation failed to recapitulate DA cell loss and did not display synuclein pathology. Mice with double mutations (A30P/A53T) in the human form of $\alpha$-synuclein exhibited a progressive loss of dopaminergic neurons in the $\mathrm{SN}$ with decreased motor activity. However, the pathological relevance is controversial since the double mutation of $\alpha$-synuclein protein has not been found in human familial PD (157).

The first conditional transgenic mouse in which the expression of $\alpha$-synuclein was regulated by the tetracycline system, has been established by Nuber et al. These mice showed a modest loss of dopaminergic neurons in the SN without any synuclein-positive inclusions. In addition, they showed reduced neurogenesis and neurodegeneration in the hippocampus without fibrillary inclusions. When $\alpha$-synuclein gene expression is turned 
off, the progression of PD-like phenotypes is alleviated, but not reversed, indicating that continuous expression of the $\alpha$-synuclein gene is necessary for disease progression (158).

Unlike the mouse model, $\alpha$-synuclein overexpression in Drosophila showed a stronger PD-like phenotype. Over-expression of mutant (A53T and A30P) or wild type $\alpha$ synuclein in flies resulted in a progressive loss of dopaminergic neurons in the dorsomedial clusters and correlated with a loss of climbing ability. In addition, $\alpha$ synuclein positive inclusions were observed in dopaminergic neurons (159).

\subsubsection{Leucine-rich repeat kinase (LRRK) 2}

The leucine-rich repeat kinase (LRRK) 2 gene contains 51 exons and encodes a large protein composed of 2527 amino acids. RNA expression occurs predominantly in the basal ganglia and the hippocampus. The protein encoded by the LRRK2 gene includes several independent domains including a leucine-rich repeat (LRR) domain, a kinase domain, a Roc GTPase domain and a C-terminal WD40 domain (160).

Mutations in LRRK2 have been recognized in approximately 7\% of familial PD cases as well as $1-3 \%$ of idiopathic PD cases. So far, at least 20 polymorphic loci in the LRRK2 gene have been identified and linked to familial PD. Among them, the G2019S missense mutation is the most common, accounting for up to $6 \%$ of familial cases in Europe (161). Unlike other PD-associated genes, the LRRK2 G2019 mutation gives rise to late-onset PD which has a clinically similar phenotype to idiopathic PD. However, the exact mechanism by which the LRRK2 mutation induces the disease is unknown (162).

In Drosophila models, over expression of either wild type or G2019S mutated LRRK2 causes adult-onset dopaminergic neuron degeneration, accompanied by decreased motor activity that is alleviated by L-DOPA treatment. In contrast to this, transgenic flies that overexpress R144C mutated LRRK showed no difference in the number of DA neurons. Y1699C or I2020T mutant flies, however, display a significant loss of DA neurons in the dorsomedial protocerebral posterior lateral (PPL1) cluster and the dorsolateral protocerebral posterior medial (PPM1/2) clusters at the age of 60 days, but not at 10 days old, suggesting that the neurotoxicity of the LRRK2 mutant requires a certain amount of time to be manifested (163)

\subsubsection{Parkin}

Mutations in parkin, a component of E3 ubiquitin ligase encoded by the PARK2 gene, is identified as the cause of autosomal recessive juvenile parkinsonism (ARJP) in humans (164). It is assumed that the loss of parkin function may impair the ubiquitinproteasome system and subsequently cause the neurotoxic accumulation of its substrates. Indeed, many substrates of parkin such as CDCrel (septin 5), Cyclin E, and far upstream element-binding protein-1 (FBP-1) were found accumulated in the brain of parkin associated PD patients, even though the DA neuron specific substrates have not been reported (162). 
Parkin null mutant mice have been generated by several independent groups. However, none of these transgenic mice exhibited typical PD pathologies. In addition, the accumulation of Parkin substrates was not detected in the brain (162). In the fly model, the parkin null mutation results in a significant and progressive loss of DA neurons in the protocerebral posterior lateral-1 (PPL-1) cluster. Moreover, the neurodegenerative phenotype of parkin mutants is more severe when combined with loss-of-function mutations of glutathione S-transferase S1 (GstS1), an important detoxification enzyme that reduces oxidative stress. A subsequent study also revealed that overexpression of GstS1 suppresses DA neurodegeneration in the parkin mutant flies, suggesting that overexpression of GstS1 has potential therapeutic value in the treatment of PD (165).

\subsubsection{Neurotoxin models}

Before the advent of genetic models, the majority of experimental PD studies relied on toxin based models. Toxin-based models recapitulate nigrostriatal neurodegeneration, however the degeneration process is often acute compared to idiopathic PD, which requires decades to develop in humans (12).

\subsubsection{1-Methyl-4-phenyl-1,2,3,6-tetrahydropyridine (MPTP)}

1-Methyl-4-phenyl-1,2,3,6-tetrahydropyridine (MPTP) as a agent that could induce parkinsonism was discovered by investigation of the "Frozen Addict" cases in 1982. A home drug developer accidentally prepared MPTP as a byproduct during the process of making a heroin derivative, MPPP (1-methyl-4-phenyl-4propionoxypiperidine), and a group of drug addicts who self-administered MPTP developed severe acute parkinsonism (17). Further studies discovered that MPTP selectively induces nonreversible dopaminergic neuron death in the SN and depletion of DA in the striatum in those patients. MPTP, a lipophilic compound, can readily cross the blood-brain barrier. Once inside the brain, MPTP is converted into the toxic metabolite, $\mathrm{MPP}+$ (1-methyl-4-phenylpyridinium) by monoamine oxidase B in glial cells. MPP+ is taken up by the dopamine transporter and accumulates in dopaminergic neurons in the $\mathrm{SN}$. Once in the neuron, MPP+ inhibits complex I of electron transport chain in mitochondria and consequently produces oxidative damage, leading to cell death (12).

In primates, MPTP causes irreversible and severe parkinsonian symptoms which are not distinguishable from those of idiopathic PD. These include the degeneration of dopaminergic neurons in the substantia nigra, LB pathology, and motor symptoms which can be ameliorated with L-dopa or dopamine agonist (17). The main drawback of the primate MPTP model is that the disease progression is more rapid than that of idiopathic PD, which develops over a few decades (166).

Rodents show variable amounts of susceptibility to MPTP toxicity, which is dependent upon the species and strain of the animal. Rats are resistant and some strains of mice are susceptible to MPTP toxicity. The C57BL/6J strain is well known as the most sensitive strain to MPTP and largely used as a MPTP induced PD model (167). However, 
little or no motor symptoms or LB pathology is observed after MPTP treatment in this strain (168).

\subsubsection{6-Hydroxydopamine (OHDA)}

6-Hydroxydopamine (OHDA) is the first chemical known to have neurotoxic effects on the catecholaminergic pathway. 6-OHDA can be directly injected either into the $\mathrm{SN}$ or striatum. Injection into the $\mathrm{SN}$ causes rapid DA cell death, while the striatal injection of 6-OHDA induces a slow retrograde degeneration of neurons in the SN. Once it is delivered, 6-OHDA is selectively accumulated in the SN neurons and presumably kills neurons by inducing free radical damage. Numerous species are sensitive to 6OHDA, including mice, cats, dogs, monkeys and rats, and a quantifiable motor deficit is present without LB pathology (166)

\subsubsection{Rotenone}

Rotenone was isolated from the roots and stems of several plants and has been used as a pesticide and fish poison. Highly lipophilic, rotenone easily penetrates the blood-brain barrier, accumulates in the mitochondria, and inhibits complex-I of the electron transport chain. Although complex-I in the mitochondria is evenly distributed throughout cells in the brain, only the dopamine neurons in the SN selectively degenerate with exposure to rotenone. This suggests that the dopamine neurons in the SN are particularly sensitive to rotenone (169).

In the rat, in addition to inducing the loss of SN DA neurons, rotenone induces formation of LB, which have not been noted in either the 6-OHDA or MPTP models. Chronic, intravenous administration of rotenone produces PD-like motor symptoms, such as bradykinesia, postural instability, and tremors. These symptoms improve with administration of L-dopa or dopamine agonist treatments (169).

However, the rotenone model does pose several disadvantages. First, PD-like pathology induced by rotenone is variable, depending on the protocol of administration of rotenone. Second, the association between PD-like motor symptoms and the loss of dopaminergic neurons has not been fully confirmed in the rotenone model. These motor changes may also be caused by the abnormalities arising in the cardiac system, stomach, and liver that are seen in the rotenone model (170).

\subsubsection{Paraquat and maneb}

Paraquat is a herbicide widely used throughout the world. Investigators are interested in paraquat, because not only does it share structural similarities with MPP+, the toxic form of MPTP, but also provides an etiological link to PD in epidemiological studies (20). Paraquat is reduced by several reductases, including mitochondrial complex $\mathrm{I}$, and becomes a PQ monovalent cation $(\mathrm{PQ}+)$. Subsequently, $\mathrm{PQ}$ free radicals are rapidly oxidized by oxygen and produce superoxide radicals and reactive oxygen species that are toxic to the cell (20). Paraquat produces a dose dependent loss of dopaminergic 
neurons in the SN and a decrease in tyrosine hydroxylase immunoreactivity in the striatum (171). The up-regulation and aggregation of $\alpha$-synuclein is also observed in the rodent PQ model (171).

Maneb is an agricultural fungicide. The mechanism of maneb toxicity is not clear, however, it is hypothesized that maneb crosses the blood brain barrier and inhibits mitochondrial complex III (172). There is a report that chronic exposure to maneb causes PD-like symptoms in humans (173). In a mouse model, maneb showed a synergistic effect on nigrostriatal degeneration when it was co-administered with paraquat. The loss of DA fibers and the turnover rate of DA and DOPAC were greater with exposure to both toxins than to either toxin administered alone $(174,175)$. This study lends support to the theory that a combination of environmental toxins may have synergistic effects on the development of PD (170)

\subsection{Viral-Induced Parkinsonism}

\subsubsection{Influenza virus}

Neurological symptoms associated with influenza have been reported as far back as 1385 and intermittent outbreaks with similar symptoms have occurred at other times during influenza outbreaks $(176,177)$. The Influenza virus has also been implicated as both a direct and an indirect cause of Parkinson's disease, based on clinical descriptions and epidemiological studies $(42,178-182)$.

Influenza viruses are negative sense, single-strand RNA viruses. Influenza viruses belong to the family of Orthomyxoviridae which comprises influenza A, B, C, Isavirus and Thogotovirus, based on differences in their nucleoprotein (NP) and matrix protein (M) (183). Influenza A and B viruses are highly contagious pathogens that cause mild to severe infections in humans. Two glycoproteins, the haemagglutinin (H or HA) and neuraminidase (N or NA), determine the antigenicity of influenza A and B. Further, Influenza A virus is clustered into sixteen $\mathrm{H}$ (H1-H16) and nine N(N1-N9) subtypes, based on antigenic analysis of the HA and NA genes (184).

Most influenza viruses attack the mucosa of the respiratory tract and are transmitted into the respiratory epithelial cells, as well as other types of cells. Replication is very fast and can occur within 6 hours after infection. Clinical symptoms include acute onset of fever, myalgias, and respiratory symptoms $(185,186)$. However, in severe cases, influenza infection may result in primary viral pneumonia, secondary bacterial pneumonia, or complications involving the central nervous system $(182,187-189)$.

\subsubsection{Viral structure}

Influenza viruses have pleomorphic shapes with an average diameter of $120 \mathrm{~nm}$. Eight RNA gene segments of influenza A encode three polymerase complexes 
(Polymerase B1, B2 and A protein), two surface glycoproteins (HA, NA), and other associated proteins including nucleoprotein (NP), matrix proteins (M1, M2), and nonstructural protein (NS). These gene segments are encapsulated by nucleoprotein (NP) (Fig 1.2) (184).

Haemagglutinin, a $76 \mathrm{kDa}$ glycoprotein, is composed of homotrimers. Each subunit spans and protrudes from the envelope membrane and is presented on the outer surface, serving as the major surface antigen of influenza virus recognized by neutralizing antibodies (190). The precursor polypeptide HA0 is synthesized and cleaved by host originated trypsin-like protease into two subunits, HA1 and HA2 (191). The head of HA1 contains the receptor binding site and binds to either alpha 2-3 or 2-6 linked sialic acid on the host cell surface, depending on the amino acid sequence in the binding pocket. The amino terminus of HA2 is very important for viral-cell membrane fusion. The cleavage of HA0 is a prerequisite for virus infectivity and is dependent upon the presence of host-originated protease. Therefore, the distribution of proteases in the tissue and the ability to cleave viral HA by the proteases are the main determinants of pathogenicity of influenza virus $(191,192)$.

Neuraminidase is a homotetramer and each monomer contains an enzymatically active globular head domain and a membrane linked stalk domain. NA cleaves the linkage between a terminal sialic acid and a D-galactose or D-galactosamine residue and has an essential role in releasing progeny viral particles from the infected cells (193, 194).

Matrix proteins, M1 and M2 are encoded by the M gene. M1 protein interacts with the cytosolic domain of HA, NA, and M2 protein and forms a layer to separate the ribonucleoprotein (RNP) complexes from the viral membrane, providing rigidity to the membrane (195). The M1 protein is also reported to regulate viral RNP nuclear transport (196). M2, a splice variant of M mRNA, is an integral membrane protein and displays ion channel activity for the regulation of $\mathrm{pH}$. This function of the M2 protein acidifies the interior of the viral particle and induces dislocation of RNP from M1 proteins and ejects RNP into the cytoplasm (196).

Basic polymerase 2 (PB2), basic polymerase 1 (PB1), and acidic polymerase protein (PA) are encoded by segments 1,2, and 3, respectively. These polymerase proteins interact with nucleoprotein (NP) and form a polymerase complex for viral transcription and replication (184). PB2 is targeted to the nucleus of the infecting cell by its nuclear localization signal. PB2 binds the 5' cap structure of viral mRNA and generates cap primers to initiate viral mRNA synthesis. PB1 also contains nuclear localization signals and is transported into the host nucleus. PB1 plays an important role in the assembly of the polymerase complex and the polymerization of RNA (197). Like PB1 and PB2, PA provides a nuclear localization signal and is packed into the nucleus of the infected cell. PA is thought to be involved in viral transcription and replication, but the exact function of PA is not fully understood (184). 


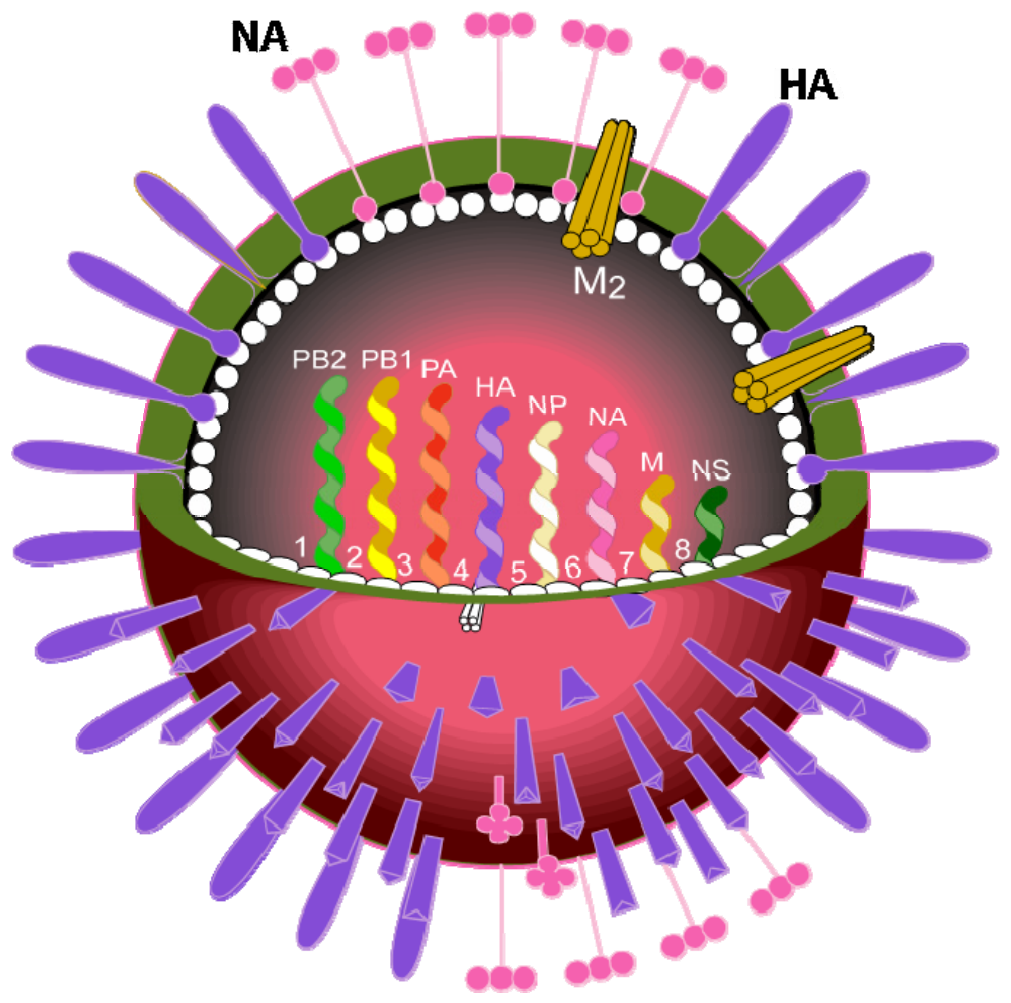

Figure 1.2 Structure of Influenza Virus

HA: Haemagglutinin, NA: Neuraminidase, M1: Matrix proteins 1, M2: Matrix proteins 2, PB1: Basic polymerase 1, PB2: Basic polymerase 2, PA: Acidic polymerase protein, NP: nucleoprotein, NS: Non structural protein.

Source: Reprinted with permission from Elsevier. Robert G. Webster. The importance of animal influenza for human disease. Vaccine 20, S16-S2012, (2002) (198). 
Nucleoprotein (NP), a phosphorylated basic protein, contains an RNA-binding domain in the amino terminus and binds to the backbone of the viral RNA. It has been suggested that NP switches viral RNA polymerase from transcription to replication based on the observation that a temperature sensitive mutant of NP synthesizes only mRNA, but not complementary RNA (cRNA), at the nonpermissive temperature. NP is also thought to be involved in nuclear transportation of viral RNA at an early phase of viral infection $(184,199)$.

Non-structural protein (NS) 1 is derived from segment 8 of the influenza A virus, while NS2 is encoded by the spliced mRNA of segment 8 . NS1 is the only known nonstructural protein at this time. It functions to inhibit splicing and nuclear export of cellular mRNA, and to enhance viral protein expression by binding to cellular RNA and viral RNA (184). Recently it has been reported that NS1 protein of A/HK/483/97(H5N1) makes the virus less susceptible to the antiviral proteins, interferon, and tumor necrosis factor alpha (200). NS2 is incorporated in viral particles. NS2 carries nuclear export signals and has an essential role when the progeny viral RNPs are exported from the nucleus to the cytoplasm of the infected cell (201).

\subsubsection{Life cycle of the influenza A virus}

The life cycle of the influenza A virus is initiated by the binding of HA to the sialic acid receptor on the host cell surface. The sialic acid linkage to galactose by $\alpha 2-3$ or $\alpha 2-6$ is the main factor that determines host specificity (202). The virus enters the host cell by clathrin-coated receptor mediated endocytosis and the vesicle harboring the whole virus then fuses with endosomes. The acidic environment of the endosome triggers a conformational change of HA, allowing the fusion of HA with the vesicular membrane. The proton influx mediated by the M2 ion channel induces dissociation of the M1 from the vRNP, resulting in uncoating of the virus. The viral RNPs are translocated to the host cell nucleus where transcription and replication occur (202).

vRNAs serve as templates for both cRNA and mRNA. PB2 polymerase recognizes and binds to the 5'end of host mRNA and cleaves it 10-15 nucleotides away from the cap structure. The viral polymerase complex uses the cleaved cap structure as primers for viral mRNA transcription (203). Viral mRNA that is capped and polyadenylated is then exported to the cytoplasm. The mRNAs encoding viral membrane proteins such as HA, NA, and M2 are translated by ribosomes in the rough endoplasmic reticulum. Subsequently, these proteins pass to the secretory pathway at the trans-Golgi for glycosylation, then move to the host cell surface and are incorporated into the host cell membrane (202). The mRNAs encoding the other proteins, PB2, PB1, PA, NP, NS1, and NS2 are translated by free ribosomes in the cytosol. PB2, PB1, PA, and NP are then transported back into the nucleus and initiate complementary RNA (cRNA) transcription using vRNAs as templates. As a full length copy of the vRNA, cRNA serves as a template for the synthesis of progeny virus genomes and newly synthesized progeny vRNA forms vRNP binding proteins. M1 proteins, which contain nuclear localization domains, then bind to vRNP and promote the nuclear export of vRNPs. NS2 protein is thought to be involved in enhancing the function of M1 protein. 
vRNPs are transported into the cell membrane where the envelope proteins are located and are composed of viral particles. When newly assembled virus particles bud off from the host cell membrane, NA cleaves the sialic acid receptors on the host cell and allows virus particles to leave the host cell (202) (Fig 1.3).

\subsubsection{Previous human influenza pandemics}

Influenza A viruses were the etiological agents of a number of pandemics in the last century including the $1918 \mathrm{H} 1 \mathrm{~N} 1$ pandemic, the $1957 \mathrm{H} 2 \mathrm{~N} 2$ pandemic, and the 1968 H3N2 pandemic. The 1918 flu pandemic, the most catastrophic pandemic, infected 500 million people, which was about $20-40 \%$ of the total world population at that time. Twenty to forty million people died with acute influenza symptoms in that single year (204). The impact was so profound that it reduced the average life expectancy in the USA by over 10 years, and of the reduction of the population was also thought to affect the end of the First World War (204).

\subsubsection{Von Economo's disease and postencephalic parkinsonism}

There is a large body of evidence that influenza can directly lead to encephalitis $(178,182,188,205-212)$. However, the link with Parkinson's disease is somewhat controversial. Much of the linkage of parkinsonism with influenza and many other viruses stems from an outbreak of encephalitis lethargica (EL), also known as von Economo's disease, and the postencephalic parkinsonism that occurred subsequent to the 1918 pandemic influenza outbreak caused by type A H1N1 influenza virus (213). It is thought that at least 500,000 cases of EL occurred from 1917 until 1940 (39).

Von Economo discussed the etiology of what he observed and ruled out external sources such as bad sausages (wurstvergiftung) and due to the timing around WWI, poison gas (gasvergiftung). He also ruled out meningitis and polio due to the fact that the patients he saw had no contact with each other, each of these diseases were independent, and no evidence of epidemics occurred as was usually seen with these two diseases. He did discuss the possibility of this being related to "grippe" which was another name for influenza. Because his cases, as well as a few others (Cruchet described a similar outbreak of "sleeping sickness" in a number of French soldiers) (214), occurred prior to the major outbreak of pandemic influenza, von Economo ultimately concluded that what he saw was a separate disorder from direct influenza infection. However, he was not able to rule out influenza as a prodromal disease. Further studies of this disease have described 28 specific subtypes of von Economo's encephalitis. Of these subtypes, only three will be relevant should another influenza pandemic occur. These three subtypes are the somnolent-opthalmoplegic type, the parkinsonian type, and the juvenile pseudopsychopathia type (215). The second type is most related to PD. The features of postencephalic parkinsonism (PEP) have both shared and distinct symptomatology to idiopathic Parkinson's disease. Similarities include many of the classic motor symptoms including bradykinesia, tremors, and some parkinsonian "mask-like" features such as 


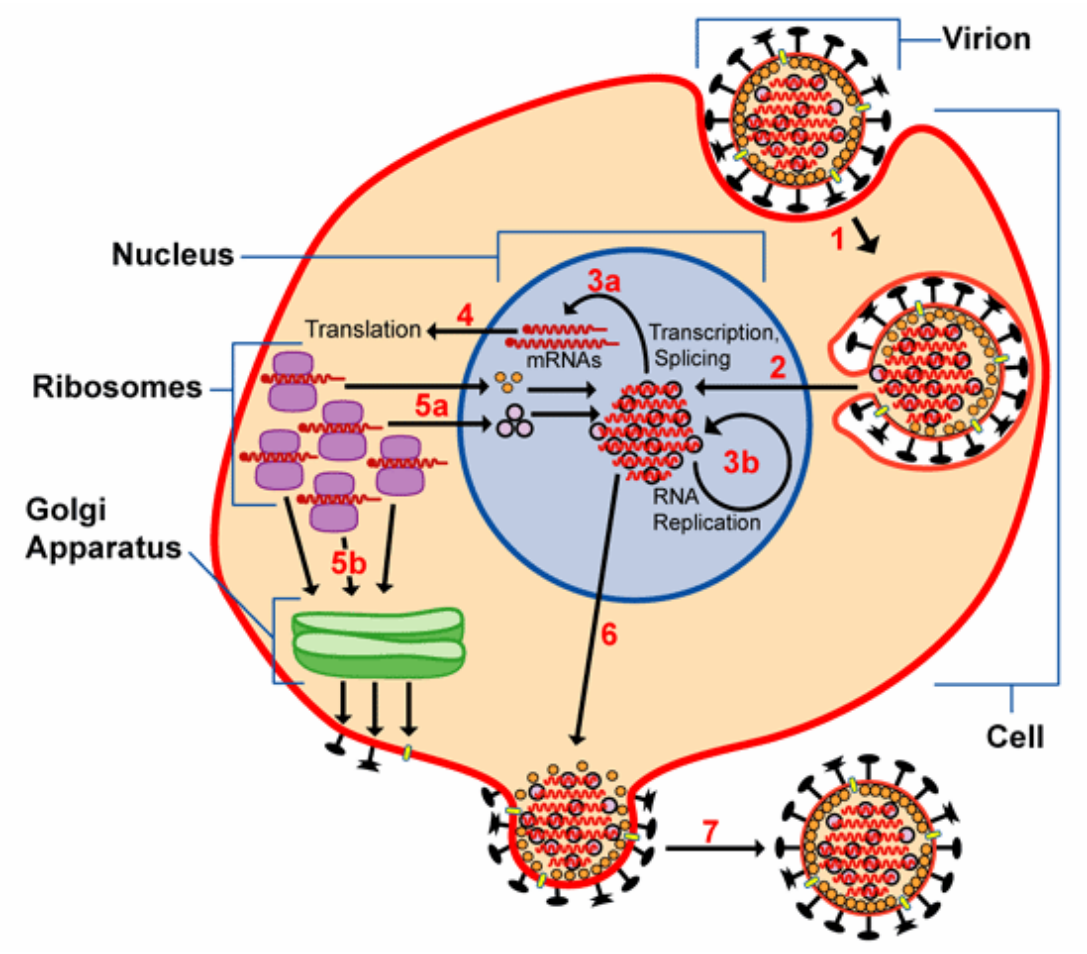

$\begin{array}{lll}\text { M1, Matrix protein 1 } & 0 & \text { M2, Matrix protein } 2 \\ \text { HA, Hemagglutinin } & \text { NA, Neuraminidase } & \text { vRNP, Viral ribonucleoprotein }\end{array}$

Figure 1.3 Life cycle of influenza virus

Source: Reprinted with permission from Bao Y., P. Bolotov, D. Dernovoy, B. Kiryutin, L. Zaslavsky, T. Tatusova, J. Ostell, and D. Lipman. The Influenza Virus Resource at the National Center for Biotechnology Information. J. Virol 82(2), 596-601 (2008)(216). 
ptosis, while differences can include facial twitching, myoclonus, catatonia, mutism, the lack of Lewy bodies, and the presence of neurofibrillary tangles that are common to Alzheimer's disease (215).

Most PEP cases occurred within 6 months to 1 year following an episode of acute EL while others developed parkinsonian symptoms after the passage of decades (217). At least $50 \%$ of PEP patients had a clinical history of acute EL (218). Epidemiological studies of PEP patients showed that PEP had a single etiology related to viral infections around the 1920s. In addition, individuals who were born between 1892 and 1929, a period of Spanish flu pandemic, had a higher incidence of PD later in life $(219,220)$. These pieces of evidence suggest a possible link between PEP and the $1918 \mathrm{flu}$ pandemic.

A causal link between PEP and the 1918 flu pandemic, however, has never been fully demonstrated and remains controversial. It has also been shown that several type A influenza viruses, travel into the nervous system following systemic infection (neurotropism) $(180,221,222)$. Related to the finding of H1N1 neurovirulence, immunofluorescent staining against antigens from two type A influenza strains have been found in the brain of a number of EL patients, suggesting that at some time, a neurovirulent form of influenza was present in the brain (36). However, influenza RNA fragments were not found in the archival brain autopsy samples of EL and PEP patients, suggesting that 1918 influenza virus was either not neurotropic or the direct cause of EL (223). In addition, the levels of anti-influenza A antibody in the serum or CSF of PD patients was not significantly different from that of age matched controls (224).

As suggested by Vilensky's writings in a report for the Sophie Cameron Trust (207), proving a negative in this case is difficult. The lack of recovery of viral RNA from EL or PEP patients is not surprising. First, and especially in the case of PEP patients, the time from infection to symptoms was many years and the viral infection would have been transient. In addition, it has been shown in many cases of encephalitis as well as toxin induced parkinsonism that the offending agent may cause a long lasting immune response in the brain that persists many years after the insult has resolved, leading to a "hit and run" mechanism where the original insult is no longer present, but the secondary sequelae persists (225).

\subsubsection{Other viruses}

\subsubsection{Coxsackie virus}

The Coxsackie virus was first isolated from human feces in the town of Coxsackie, New York, in 1948 by G. Dalldorf $(226,227)$. The Coxsackie virus is a member of the Picornaviridae family of viruses in the genus termed Enterovirus. According to the Center for Disease Prevention and Control, there are 66 serotypes of enterovirus and these include 3 polioviruses. Coxsackie viruses are RNA viruses that primarily affect children and young adults (228). Infection with Coxsakie virus can easily 
be passed from person to person and been associated with a number of diseases, including meningitis (229), myocarditis (230), and pericarditis (231).

Acute parkinsonism has been noted after infection with Coxsackie virus $(48,49)$, although an association with traditional adult-onset idiopathic Parkinson's disease has never been established. As with the influenza virus, it is possible that early infection with the Coxsackie virus can induce a long lived activation of glial cells that would predispose the subject to succumb to oxidative insult much later in life (230).

\subsubsection{Japanese encephalitis B, St. Louis and West Nile viruses}

Japanese encephalitis B (JEBV), St. Louis, and West Nile virus are single strand DNA viruses that are transmitted by the bite of culicine mosquitoes (232-234). Although infection with these three viruses are often resolved prior to any CNS involvement, on rare occasion, these viruses can lead to encephalitis (235). In fact, JEBV is the most common cause of encephalitis in Asia (236). If infection does involve the CNS, the regions of the brain noted to become involved include the thalamus, basal ganglia, brain stem, cerebellum, hippocampus, and cerebral cortex (237-239). Ogata et al experimentally infected Fisher rats with JEBV and noted a marked gliosis in the SNpc, in a pattern typical of the lesions seen in Parkinson's disease (64). Behaviorally the rats exhibited bradykinesia that was reversed with administration of L-DOPA and a MAO inhibitor, suggesting that the virus had the ability to directly induce one of the cardinal symptoms of Parkinson's disease.

Parkinsonism secondary to St. Louis encephalitis has also been described. Like JEBV, this virus primarily affects children and the aged, although numerous cases of infection and secondary parkinsonism have been reported in all age groups. Pranzatelli et al reported a number of cases of secondary parkinsonism in children, one of which appeared to be the result of active St. Louis encephalopathy (68). In this case, the patient had features of moderate parkinsonism (The Unified Parkinson's Disease Rating Scale score of 92 and The Modified Hoen and Yahr staging of 5) with predominant symptoms of dysphasia and dystonic posture. The parkinsonism symptoms did not progress and resolved after a few months. During the clinical course of the parkinsonism, an MRI revealed a slight enhancement of the basal ganglion. In the adult cases, each patient presented with involvement of the substantia nigra as determined by an MRI (67). The first patient was a 21-year-old male who presented with a 1-week history of fever and headache. Neurologic examination was normal, and an admitting diagnosis of aseptic meningitis was made. The symptoms progressed with new symptoms of fever, ataxia, nystagmus, and tremulousness. MRI imaging revealed a T2-weighted bilateral hyper intensity in the substantia nigra without enhancement. In a second case, a 37-year-old male with a history of paranoid schizophrenia and seizures presented with fever and confusion and was generally unresponsive to commands. Other symptoms included diffuse nuchal rigidity, generalized hypertonicity, and abnormal postures including flexed upper extremities, a bilateral Babinski response, and an absent gag reflex. Like the previous patient, MRI imaging showed an asymmetric T2-weighted non-enhanced hyper- 
intensity in the substantia nigra. Similar lesions have been reported in other cases of St. Louis encephalopathies.

\subsubsection{Human immunodeficiency virus (HIV)}

Human immunodeficiency virus (HIV, originally called HTLV) is a retrovirus that has been shown to be the cause of acquired immunodeficiency syndrome (AIDS) (240). Infection with HIV results in a failure of the immune system, leading to lifethreatening opportunistic infections. The underlying cellular lesion in HIV is a progressive loss of CD4 + T cells whose levels inversely correlated with the viral load. In addition to the loss of the peripheral immune system, one of the most commonly associated pathologies from HIV infection involves motor disturbances $(241,242)$. The involvement of the CNS can occur quickly since HIV has been detected in the brain within two weeks of the initial infection (243). Once in the brain, HIV can infect the three major cell types: neurons, astrocytes, and microglia (244-246). Depending on the size of the study cohorts, it has been estimated that from 5-50\% of all AIDS patients suffer from some sort of motor dysregulation including those seen in Parkinson's disease such as bradykinesia, cogwheel rigidity, and tremors (242). These movement disorders result from both primary HIV infection and secondary opportunistic infections. Primary HIVassociated parkinsonism often appears within several months of the diagnosis of HIV infection (241) and its appearance portends a poor prognosis. Cerebral imaging (CT and MRI) of HIV-induced parkinsonism has shown lesions at various levels of the basal ganglia including calcifications throughout the basal ganglia, hypodense lesions of the striatum $(247)$, putamen hypertrophy $(248,249)$ as well as intensifying lesions of the basal ganglia $(250,251)$ and midbrain $(252)$. Functional imaging using [18F] fluorodeoxyglucose PET have shown that early CNS changes in AIDS involve thalamic and basal ganglia hyper metabolism while cortical and subcortical gray matter hypo metabolism was more characteristic of later CNS changes (253).

\subsection{Current H5N1 Influenza Panzootic}

Currently, the highly pathogenic type A influenza virus (H5N1) is thought to be a pandemic threat. Although transmission from bird to human is rare, the outbreak of H5N1 virus in Hong Kong in 1997 demonstrated that direct transmission from bird to human without genetic reassortment was possible (254). So far, over 436 human cases with a fatality rate of approximately $50 \%$ have been reported (255). Because the H5N1 influenza virus has never been associated with influenza outbreak in humans and there is no acquired immunity, and the chance of this becoming pandemic is great if the virus acquire the ability to transmit from human to human. The World Health Organization (WHO) warned that should H5N1 become pandemic in humans (255) there will likely be 2 to 7.4 million deaths globally with $134-233$ million outpatient visits and 1.5-5.2 million hospital admissions. 


\subsubsection{Evidence of neurotropism}

The highly pathogenic H5N1 influenza virus, which currently has pandemic potential, has been shown to be neurotropic $(181,221,256))$. Animal populations infected with $\mathrm{H} 5 \mathrm{~N} 1$ demonstrate clear motor effects that include abnormal postures, difficulty in maintaining an upright posture, and inability to initiate movement $(181,221$, 256). The onset of post-influenza encephalopathies are not limited to animals as there is one case report of human exposure to $\mathrm{H} 5 \mathrm{~N} 1$, a 4 year old boy and his 9 year old sister, both of whom presented with rapid encephalopathy followed by coma and death (207); its rapid course and lack of resources did not allow MRI studies to be performed. In addition to this one published case report, there are other reports of post-H5N1 influenza infection encephalitis, including a 67-year-old woman from Indonesia's West Java province who, in addition to her severe respiratory symptoms, developed encephalitis (257).

In a study examining neurovirulence in mice, Lipatov et al (181) examined 5 influenza viruses isolated in Hong Kong in 2001, of which 4 were neurotropic (Ck/HK/YU822.2/01, Ph/HK/FY155/01, Ck/HK/FY150/01, Ck/HK/NT873.3/01) and 1 was not (Ck/HK/YU562/01). This group did extensive sequence analysis of the viruses and found that there was not a common set of mutations that induced neurotropism, suggesting that there were different ways for these influenza viruses to become neurotropic. They found that multiple basic cleavage sites in the surface hemagglutinin proteins were necessary, but not sufficient, to make these viruses neurovirulent. In addition, they also suggested that specific changes in polymerase proteins PB2 and PA, which are important in transcription and replication of viral RNAs (258), are also implicated in this process. Also, several studies have found that mutations in the Mx gene, which regulates GTPase activity $(259,260)$ and acts as an important downstream effector of interferon (261), also regulates Type A influenza neurotropism. It is not known if there are any genetic differences that may confer resistance to the neurotropic behavior of $\mathrm{H} 5 \mathrm{~N} 1$ in mice (i.e. the animals can be systemically affected by virus, but be protected from nervous system involvement).

\subsubsection{The possible routes of entry into the central nervous system (CNS)}

It has been demonstrated that the A/WSN/33 strain of influenza virus enters the CNS via the olfactory epithelium and nerve (CN 1) (262). It has also been hypothesized that it can enter the CNS through other cranial nerves, including the vagus and trigeminal nerves (263-266). These three nerves have processes that innervate visceral organs and tissues that would be the first contacted by an intranasal viral infection such as the olfactory epithelium (olfactory nerve, $\mathrm{CN}$ I), orofacial mucosa (trigeminal nerve, $\mathrm{CN} \mathrm{V}$ ) and digestive system (Vagus nerve, $\mathrm{CN} \mathrm{X}$ ). The basis of this hypothesis, which does not have any direct proof such as isolation of the virus from axons of these nerves, is twofold. First, examination of the CNS following infection via intranasal routes shows that the virus is first seen in the regions innervated by these nerves. Second, the virus can be detected (indirectly by the presence of immunohistochemical detection of viral nuclear 
protein, anti NP) in the visceral ganglia $(256,266)$. One argument against this route of entry into the CNS is that the A/WSN/33 strain of influenza has an affinity for the substantia nigra, a neuronal population without any direct anatomical connection to the cranial nerve system (267). This suggests that the influenza virus may also enter into the CNS via different mechanisms of axonal transport such as through the ependymal cells lining of the ventricles and shedding into the CSF. Here, it can freely transmit through the whole neuraxis, through the blood and extravasation from penetrating capillaries in the brain or invasion into the CNS through attenuation of the blood-brain-barrier around the circumventricular organs.

\subsubsection{Scope and objective of dissertation}

Preliminary data from our laboratory revealed that the H5N1 virus is neurotropic and infects multiple brain nuclei, including the substantia nigra pars compacta (SNpc). With the following specific aims, we determine if H5N1 viral infection can either directly or indirectly induce a parkinsonian pathology.

\subsubsection{Specific aim 1}

Determine the temporal and spatial localization of $\mathrm{H} 5 \mathrm{~N} 1$ influenza virus infection in the CNS after intranasal inoculation. The highly pathogenic $\mathrm{H} 5 \mathrm{~N} 1$ virus (A/VN/1203/04) will be administered intranasally to seven week old female C57BL/6J

mice at doses of $10^{2}$ EID $_{50}$. Brains and spinal cords will be harvested at 1, 3, 7, 10, 21, 60 and 90 days post-infection, frozen and serially sectioned. Immunostaining will be utilized to assess the time course of infection, map the temporal and spatial localization of the influenza virus, and to determine the route of viral infection into the CNS.

\subsubsection{Specific aim 2}

Determine if the highly pathogenic H5N1 influenza virus infection in the CNS results in pathological changes that lead to neurodegeneration and parkinsonian pathology. Seven week old female C57BL/6J mice will be exposed to the H5N1 virus (A/VN/1203/04) as described above. Brains and spinal cords will be harvested at 1, 3, 7, 10, 21, 60 and 90 days post-infection. Samples will be processed for immunohistochemistry. Immunostaining and western blot analysis will be performed to assess the extent of neurodegeneration and parkinsonian pathology.

\subsubsection{Specific aim 3}

Determine if the highly pathogenic H5N1 influenza virus infection affects the anatomy and pharmacology of the basal ganglia and other systems, known to be affected in Parkinson's disease. Seven week old female C57BL/6J mice will be exposed to H5N1 virus (A/VN/1203/04) as described above. The SNpc and striatum will be collected from at least 4 animals at the time points 1, 10, 21, 60 and 90 days post-infection. Using 
stereology, the SNpc will be assessed to determine the number of tyrosine hydroxylase (TH) positive dopaminergic neurons. The striatal dopamine (DA), 3, 4-dihydroxyphenylacetic acid (DOPAC) and homovanillic acid (HVA) will be measured by high performance liquid chromatography (HPLC).

\subsubsection{Specific aim 4}

Determine if prior viral infection with $\mathrm{H} 5 \mathrm{~N} 1$ increases CNS vulnerability to the known parkinsonian toxin paraquat. Seven week old female C57BL/6J mice will be exposed to H5N1 virus (A/VN/1203/04) as described above. Mice surviving the viral challenge and age matched non-infected controls will be injected with paraquat (PQ) using low $(5 \mathrm{mg} / \mathrm{kg})$ concentrations of paraquat hydrochloride, every 3rd day for 21 days, starting on day 60 after inoculation. Saline will be administered for control groups. The number of TH-positive dopaminergic neurons will be assessed by stereological methods. The amount of striatal DA, DOPAC, and HVA will be measured by HPLC. 


\section{CHAPTER 2. HIGHLY PATHOGENIC H5N1 INFLUENZA VIRUS CAN ENTER THE CENTRAL NERVOUS SYSTEM AND INDUCE NEUROINFLAMMATION AND NEURODEGENERATION*}

\subsection{Introduction}

At the time of initiation of this project, the greatest influenza pandemic threat was posed by the highly pathogenic H5N1 avian influenza virus. To date, $63 \%$ of the 436 known human cases of H5N1 infection have proved fatal (255). Animals infected by $\mathrm{H} 5 \mathrm{~N} 1$ viruses have demonstrated acute neurological signs ranging from mild encephalitis to motor disturbances to coma $(180,268-270)$. However, no studies have examined the longer-term neurologic consequences of H5N1 infection. Using the C57BL/6J mouse, a strain that can be infected by the A/VN/1203/04 H5N1 virus without adaptation, we show that this virus travels from the peripheral nervous system into the CNS to higher levels of the neuroaxis. In regions infected by $\mathrm{H} 5 \mathrm{~N} 1$ virus, we observe activation of microglia and alpha-synuclein phosphorylation and aggregation that persists long after resolution of the infection. We also observe a significant, but temporal reduction of tyrosine hydroxylase positive dopaminergic neurons in the substantia nigra pars compacta, at 60 days postinfection. Our results suggest that a pandemic $\mathrm{H} 5 \mathrm{~N} 1$ pathogen, or other neurotropic influenza virus, could initiate CNS disorders of protein aggregation including Parkinson's diseases.

\subsection{Materials and Methods}

All experimental infection of animals and handling of live H5N1 viruses were performed in a Biosafety level 3+ laboratory approved for use by the U.S. Department of Agriculture and exclusively utilized by the Division of Virology and other approved scientists at St. Jude Children's Research Hospital for highly pathogenic avian influenza work.

\subsubsection{Virus stock preparation and inoculation of mice with $\mathrm{H} 5 \mathrm{N1}$}

Stock viruses were prepared by propagating neurotropic A/VN/1203/04 (H5N1) influenza virus for one passage in the allantoic cavity of 10-days-old embryonated chicken eggs for 40 to 48 hours at $37^{\circ} \mathrm{C}$. Viral infectious titers were determined using the method of Reed and Muench (271), and expressed in $\log 10$ of the 50\% egg infectious dose per $1.0 \mathrm{ml}$ of fluid $\left(\mathrm{EID}_{50} / \mathrm{mL}\right)$.

* Chapter 2 adapted with permission. Jang H, Boltz D, Sturm-Ramirez K, Shepherd KR, Jiao Y, Webster R, Smeyne RJ. Highly pathogenic H5N1 influenza virus can enter the central nervous system and induce neuroinflammation and neurodegeneration. Proc Natl Acad Sci 106 (33), 14063-8 (2009) (270). 
6-8 week old C57BL/6J mice (Jackson Labs, Bar Harbor, ME) were anesthetized by isofluorane inhalation and infected intranasally with $30 \mu \mathrm{L}$ of allantoic fluid diluted in PBS to the target virus infectious titer $\left(10^{2}\right.$ EID $\left._{50}\right)$.

\subsubsection{Immunocytochemistry}

1, 2, 3, 7, 10, 21, 60 and 90 days following intranasal inoculation of H5N1, mice were deeply anesthetized with Avertin and transcardially perfused with $0.9 \%$ saline followed by $10 \%$ neutral buffered formalin. Brains and visceral organs were removed and postfixed for 3 weeks in $10 \%$ neutral buffered formalin to ensure that virus particles present in the tissue have been killed. The tissue was subsequently cryoprotected in $30 \%$ sucrose/phosphate buffered saline (PBS), serially-sectioned at $20 \mu \mathrm{m}$, and mounted onto polyionic slides (12-550, Superfrost-plus, Fisher, Pittsburgh, PA). H5N1 influenza virus was detected with a pool of four monoclonal antibodies raised against the nuclear protein (NP) of influenza virus A/WSN/33 (H1N1) (272). The monoclonal antibodies target four different antigenic epitopes of the NP protein and detect all known subtypes of influenza A viruses. Three different animals were used to determine the pattern of H5N1 influenza virus expression at each time point. $\mathrm{H} 5 \mathrm{~N} 1$ influenza virus location was mapped using images generated from an electronic atlas (273) and a CNS nucleus template (274). The volume of virus was scored in a semi-quantitative manner that has been detailed (274).

Antibodies directed against glial fibrillary acidic protein (GFAP) (rabbit, G4546, Sigma, St. Louis, MO, 1:500) were used to detect astrocytosis and antibodies directed against ionized calcium binding adaptor molecule -1 (Iba-1) (rabbit, 019-19741, Wako Chemicals, Richmond, VA, 1:500) was used to detect microgliosis (12). Activated caspase-3 (rabbit, 559565, BD Biosciences, San Jose, CA) and Fluoro Jade B (AG310, Chemicon, Temecula, CA) staining to mark apoptotic and necrotic cells, respectively, were examined as previously described (275). Antibodies directed against tyrosine hydroxylase (TH) (rabbit, P40101-0, PelFreeze, Rogers, AK, 1:500) were used to detect dopaminergic neurons. Alpha synuclein (mouse, 610786, BD Biosciences, San Jose, CA, 1:1000) and pSer129 SYN (mouse, 014-20281 WAKO, Richmond, VA, 1:1000) immunocytochemistry were performed on frozen sections using standard protocols (12). Transmission electron microscopy was performed as previously described (276)

\subsubsection{Dorsal root ganglia (DRG) neuron culture and microfluidic chamber studies}

Dissociated dorsal root ganglia (DRG) neurons were prepared as previously described (277). Briefly, DRG neurons were dissected from 6 to 8 weeks old female C57BL/6J mice and incubated in 60 units of papain (3126, Worthington, Freehold, NJ) /Hank's balanced salt solution (HBSS) (14170, GIBCO, Carlsbad, CA) for $10 \mathrm{~min}$ at $37^{\circ} \mathrm{C}$. Subsequently, 12mg of collagenase (4176, Worthington, Freehold, NJ) and $100 \mu \mathrm{L}$ of dispase (10269638001, Roche Diagnostics, Indianapolis, IN) were added and reacted with dissociated DRG neurons for $10 \mathrm{~min}$ at $37^{\circ} \mathrm{C}$. The pellet was washed twice with F12/10\% FBS medium (11765 GIBCO, Carlsbad, CA) to remove enzymes and given 
$0.5 \mathrm{ml}$ fresh medium. The ganglia were triturated 10 times using a fire-polished Pasteur pipette. 60,000 dissociated cells within $20 \mu \mathrm{L}$ were plated into the somal compartment of microfluidic chambers (SND150, Xona Microfluidics LLC, Aliso Viejo, CA) (278) which is placed on a cover glass previously coated with laminin (L2020, Sigma, St. Louis, MO) and poly-D-Lysine (BD354210, BD Biosciences, San Jose, CA). After 30 minutes to allow cell attachment, cultures were rinsed and 150 microliters of fresh culture medium containing $10 \mathrm{ng} / \mathrm{ml}$ nerve growth factor (NGF 2.5s, N6009, Sigma, St. Louis, $\mathrm{MO})$ was added to the process compartment of the microfluidic chamber. Cultures were maintained in a humidified $37^{\circ} \mathrm{C}$ chamber with $5 \% \mathrm{CO}_{2}$ for 12 days and the culture medium was changed every three days.

$2 \times 10^{6}$ plaque-forming unit (PFU) of H5N1 (VN/1203/04) virus in 20 microliters serum free medium was loaded in the axonal side of the compartment. After one hour, the microfluidic axonal chamber was washed 2 times by adding $130 \mu \mathrm{L}$ of serum free media to the top reservoir and collecting the flow through in the bottom reservoir. $130 \mu \mathrm{L}$ of medium with serum was then added to the top and bottom reservoirs on the axonal side to maintain the hydrostatic balance. At the $1 \mathrm{~h}, 24 \mathrm{~h}, 48 \mathrm{hr}$ and $72 \mathrm{hr}$ after infection, cells were rinsed with 1X PBS twice and fixed by $4 \%$ paraformaldehyde for $10 \mathrm{~min}$ followed by post fixation for 2 weeks to kill residual virus or viral particles. Virus was detected by immunohistochemical methods as described above. Antibodies directed against neuronspecific $\beta$-III tubulin (rabbit, ab18207, Abcam, Cambridge, MA, 1:500) were used to label cell bodies and processes of DRG neurons. Images were captured by using a Leica SP1 upright laser scanning confocal microscope.

\subsubsection{Quantification of phosphorylated $\alpha$-synuclein expression}

To ensure consistency in this analysis, all sections were processed on the same day using the same solutions and antibody preparations, including lot numbers. $16 \mu \mathrm{m}$ coronal frozen sections were washed with $0.1 \mathrm{M}$ PBS. One set of sections were incubated with $10 \mu \mathrm{g} / \mathrm{mL}$ proteinase $\mathrm{K}$ (25530, Invitrogen, Carlsbad, CA)/0.1M PBS solution for $10 \mathrm{~min}$ at room temperature, and another set of adjacent sections were washed with $0.1 \mathrm{M}$ PBS without proteinase K. These different methods specifically allow identification of insoluble versus soluble pSer129SYN, respectively (56). All sections were blocked with the Mouse-on-Mouse (M.O.M) blocking solution (PK-2200,Vector Laboratories, Burlingame, CA) for $1 \mathrm{~h}$ and incubated overnight at $4^{\circ} \mathrm{C}$ with anti-mouse phospho Ser129 antibody (mouse, 014-20281,WAKO, Richmond, VA, 1:1000) diluted in M.O.M diluent solution. Sections were incubated with a biotinylated secondary antibody for 30min and then incubated with avidin-biotin complex (PK-6102,Vector Laboratories, Burlingame, $\mathrm{CA}$ ) for $15 \mathrm{~min}$ and washed 5 times with $0.1 \mathrm{M}$ PBST for $5 \mathrm{~min}$ each and developed with 3-3' diaminobenzine (DAB) (D-5905, Sigma, St. Louis, MO) as the chromogen. Sections were rinsed with tap water and dehydrated, cleared and mounted with Permount (SP15500, Sigma, St. Louis, MO). Images were captured at 10x using the virtual slice program in the Microbrightfield system (Microbrightfield, Williston, VT) attached to an Olympus BX60 microscope. All images for analysis were taken at the same light intensity at one sitting to control for variations in bulb intensity and camera chip differences. All images 
were saved as a tif file and optical densities of the rostral hippocampus, olfactory bulb, locus coeruleus, solitary nucleus and cerebellum were obtained on raw images using the NIH image program (ImageJ, version 1.43, National Institute of Health, Bethesda, MD). The density of the background from each section was also obtained and subtracted from the region density to give a final number. Statistical analysis was performed using GraphPad statistical software (Prism, version 4.03, GraphPad Software, La Jolla, CA).

\subsubsection{Stereological assessment of SNpc dopaminergic neuron number}

The total number of tyrosine hydroxylase positive neurons in both hemispheres of SNpc was estimated using the optical fractionator method (279) from Stereo Investigator (version 7.0, MicroBrightField, Colchester, VT). The broad outlines of the SNpc in both hemispheres were delineated at low power (4× magnification). An unbiased counting frames $(60 \times 60 \mu \mathrm{m})$ placed at the intersections of a grid (frame size $200 \times 200 \mu \mathrm{m})$ were randomly superimposed on a video image of the contoured sections. Sections were examined under high power (100× magnification) objective lens on a BX51 microscope (Olympus, Center Valley, PA) with a MAC5000 motorized XYZ axis computercontrolled stage and a CX9000 CCD video camera (MicroBrightField, Colchester, VT). TH-positive neurons were counted at the depth that their nucleus is focused, in each counting area. The reliability of the estimates were measured by calculation of the coefficient of error (279). Gundersen coefficients of error for $m=1$ were all less than or equal to 0.10. Statistical significance was calculated using a one-way ANOVA followed by Student-Newman-Keuls post hoc test (280).

\subsection{Results}

\subsubsection{Body weight loss in H5N1 virus infected mice}

We intranasally inoculated the highly pathogenic and neurotropic A/VN/1203/04 (H5N1) virus to 1636 to 8 week old C57BL/6J mice (Jackson Laboratories, Bar Harbor, $\mathrm{ME}$ ) with the $10^{2}$ egg infection dose $\left(\mathrm{EID}_{50}\right)$ (281) and monitored the mortality (Fig 21A) and the body weight loss of infected mice (Fig 2-1B). Approximately $60 \%$ of mice died between 9 and 13 days post infection, recording $60 \%$ morality rate which is close to that seen in human cases (Fig 2-1A). Six days post-infection (dpi), 88\% of the mice demonstrated weight loss; by day 9 dpi, weight loss was greater than $10 \%$ of initial body weight. All of the infected animals that showed weight loss showed neurological signs including ataxia, tremor and hind leg paralysis. Approximately half of these animal's symptoms became so severe that they had to be euthanized. The remainder showed milder neurological signs and recovered by day 21 post inoculation (Fig. 2-1B). 
A

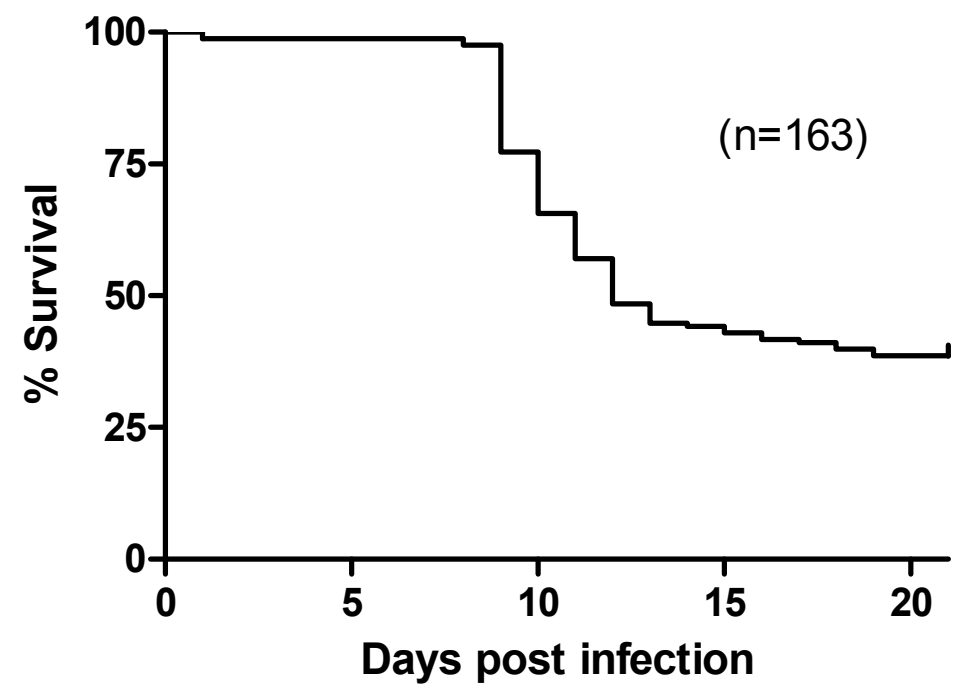

B

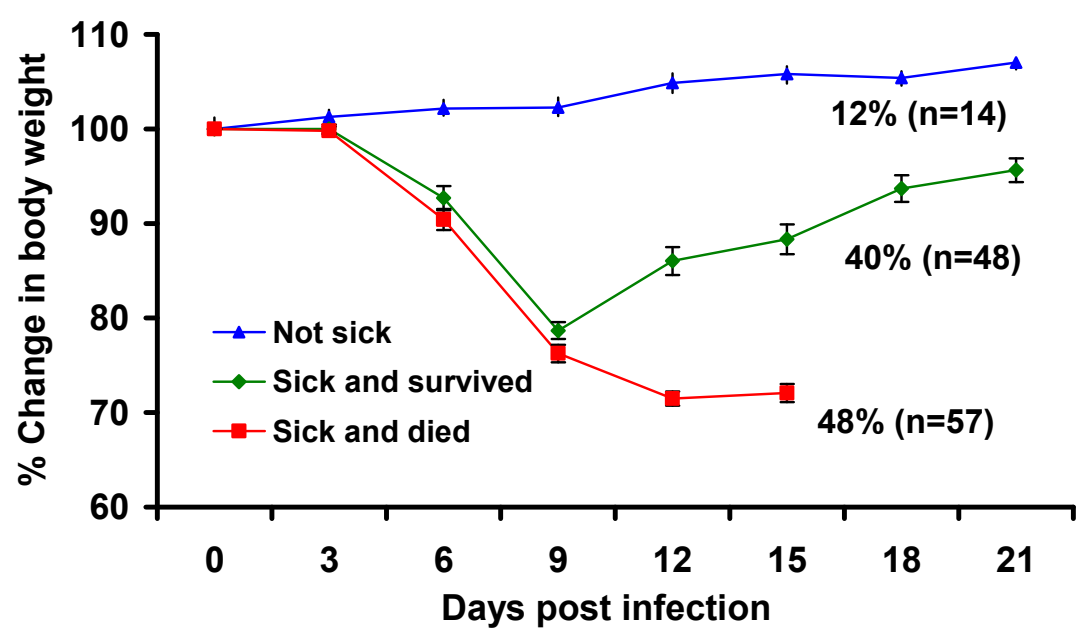

Figure 2.1 Percent Survival and Body Weight Change in the Infected Animals

About half of $\mathrm{H} 5 \mathrm{~N} 1$ infected C57BL/6J mice died between 9-12 days post infection, representing $60 \%$ of mortality rates (A). Among these populations, $14 \%$ of mice didn't show any signs of infection with absence of body weight loss (Blue colored). $40 \%$ of mice showed around $10-20 \%$ of weight loss with or without neurological symptoms including hind leg paralysis, tremor, and lethargy around 10 days post infection and then gradually gained weights and recovered (Green colored). $48 \%$ of mice showed more than $20 \%$ body weight loss from the initial body weight, presenting neurological symptoms, including hind leg paralysis, tremor, and lethargy, and died around days 10-15 days post infection (B). Error bars represent the standard error of mean (s.e.m). 


\subsubsection{Spatial and temporal distribution of H5N1 virus}

The neurological signs observed in the infected mice suggested involvement of several levels of the neuraxis. We mapped the progression of the $\mathrm{H} 5 \mathrm{~N} 1$ virus through the nervous system by using immunohistochemical detection of the H5N1 nucleoprotein (NP) (Table 2.1). H5N1 virus was first detected on day 2-3 dpi in the myenteric (Auerbach's) plexi of the enteric nervous system (Fig. 2.2A) and in neurons within the dorsal root ganglia (Fig. 2.2B) of the peripheral nervous system. H5N1 virus was first observed in the CNS on day 3 dpi in the brainstem solitary nucleus (Fig. 2.2C), which receives primary afferent signals from various visceral regions and organs, including lung and gut (282). On day $7 \mathrm{dpi}$, virus was found in the vagal and hypoglossal brainstem nuclei and in the midbrain locus coeruleus and SNpc (Fig, 2.2D). Outside of the brainstem and midbrain, H5N1 virus was detected in the periglomerular and mitral (Fig. 2.2E) cells of the olfactory bulb and in neurons within the spinal cord (principally in the thoracic region) (Fig. 2.2F). On day $10 \mathrm{dpi}, \mathrm{H} 5 \mathrm{~N} 1$ infection had spread to all levels of the central neuraxis but did not involve all structures (Fig 2.3) (Fig 2.4). By day 21, the surviving animals displayed no visible neurological signs and H5N1 virus was not detected in the CNS. The pattern of infection in lung and brain suggested that active infection lasted approximately 10 days, as described in humans (207).

\subsubsection{Cell type specific H5N1 virus infection}

In the CNS, the virus was detected in neurons (Fig. 2.2G) and microglia (Fig. 2.2H) but was not seen in astrocytes (Fig. 2.2I). The presence of virus in brainstem neurons was supported by transmission electron microscopy, which revealed structures of the shape and size characteristic of H5N1 influenza virus (283) in the cytoplasm (Fig. 2.2J) and nucleus.

\subsubsection{Retrograde transport of H5N1 virus in vitro}

Our observation of $\mathrm{H} 5 \mathrm{~N} 1$ virus first in the enteric and peripheral nervous systems and then slightly later in the CNS, first in the solitary brainstem nucleus and subsequently in other brainstem nuclei (Table 1, Fig 2.1-4), suggests that the A/VN/1203/04 virus may enter the CNS via cranial nerves, a mechanism used by other strains of $\mathrm{H} 5 \mathrm{~N} 1(17,18)$. This hypothesis was tested by using Microfluidic chambers that allow neuronal cell bodies and their processes to be grown in one compartment while their processes extend through $150-\mu \mathrm{m}$ wide microgrooves into a separate process compartment (278). We added $\mathrm{H} 5 \mathrm{~N} 1$ virus to the process compartment for 1 hour and detected virus in the cell bodies (Fig. 2.5A) and their processes (Fig. 2.5B) within the following 24 hours. Because hydrostatic pressure differences between chambers would have confined extracellular virus to the process compartment, this finding showed that the virus detected in neuronal soma had been transported intracellularly. Direct exposure of cell bodies to H5N1 influenza also resulted in infection (Fig. 2.5C). These results demonstrate that H5N1 
Table 2.1 Semi-Quantitative Analysis of H5N1 Expression in the CNS and Lung

\begin{tabular}{|c|c|c|c|c|c|c|c|c|c|c|c|c|c|c|}
\hline Dpi & Lung & Ctx & OB & $\begin{array}{l}\text { DRG of thoracic } \\
\text { level }\end{array}$ & Thal & SNpc & $\mathbf{L C}$ & $\mathbf{R N}$ & Vest n & Pons & Solitary n & VII & $\mathbf{X}$ & XII \\
\hline 1 & + & - & - & ND & - & - & - & - & - & - & - & - & - & - \\
\hline 3 & ++ & - & - & + & - & - & - & - & - & - & + & - & - & - \\
\hline 7 & ++ & - & + & ND & - & + & + & - & + & - & ++ & - & ++ & ++ \\
\hline 10 & + & ++ & ++ & ++ & ++ & ++ & ++ & + & ++ & ++ & +++ & ++ & ++ & +++ \\
\hline 21 & - & - & - & ND & - & - & - & - & - & - & - & - & - & - \\
\hline 60 & - & - & - & ND & - & - & - & - & - & - & - & - & - & - \\
\hline 90 & - & - & - & ND & - & - & - & - & - & - & - & - & - & - \\
\hline
\end{tabular}

Semiquantitative immunohistochemical measurement of the percentage of anti-NP-positive cells at the indicated days post infection with $\mathrm{H} 5 \mathrm{~N} 1$ virus,,$+ 0 \%-33 \%$; ++, 34\%-67\%; +++, 67-100\%; -, none, ND, not determined; Ctx, cerebral cortex; OB, olfactory bulb; DRG, dorsal root ganglia; Thal, thalamus; SNpc, substantia nigra pars compacta; LC, locus coeruleus; RN, red nucleus; Vest n, vestibular nucleus; Solitary n, solitary nucleus; VII, facial nucleus; X, vagal nucleus; XII, hypoglossal nucleus. 
Figure 2.2 Localization of H5N1 Influenza Virus in the Nervous System

(A), 3 days post-inoculation (dpi) with H5N1, the virus is detected in Auerbach's plexus of the enteric nervous system (arrow) by using an antibody to the viral nucleoprotein (NP). (B), In the thoracic dorsal root ganglia, viral NP is first detected on day $3 \mathrm{dpi}$; by day 10 dpi, virtually all large and medium-sized neurons (arrows), but few small neurons, are NP-positive. (C), At day 3 dpi, NP protein (red) is first detected in the neurons (white arrows) of the brainstem solitary nucleus. Blue stain is DAPI. (D), At day 7 dpi, NP protein (green; arrows) is detected in dopaminergic neurons in the SNpc (red, anti-TH). (E), At day 7 dpi, NP protein (brown) is found in the periglomerular cells (arrows) and mitral cells (arrowheads) of the olfactory bulb. (F), At day $10 \mathrm{dpi}$, NP protein is found in the spinal cord, primarily at the thoracic level. High levels of NP are seen around Clarke's column (arrow) and the intermediolateral cell column (arrowhead). (G), NP protein (red) is found in the nuclei of neurons in the brainstem (blue, DAPI; green, antibeta3-tubulin). (H), NP protein (red) is found in the nuclei and cytoplasm of microglia (blue, DAPI; green, anti-Iba-1; areas of colocalization are yellow). (I), NP protein (red; arrows) is not observed in astrocytes (green, anti-GFAP). (J), Transmission electron micrograph of a neuron in the brainstem shows a virus particle of the shape and size characteristic of $\mathrm{H} 5 \mathrm{~N} 1$ in the cytoplasm (arrow). The mitochondrion (arrowhead) shows relative size. (K), Transmission electron micrograph of an axon traversing the brainstem shows virus particles of the characteristic shape and size of H5N1 in the axoplasm (arrow). Arrowheads indicate the myelin sheath surrounding the axon. Scale bars (microns): A) 20, B) 50, C) 25, D) 45, E) 50, F) 200, G) 10, H) 6, I) 12, J) 0.200, K) 0.120 . 

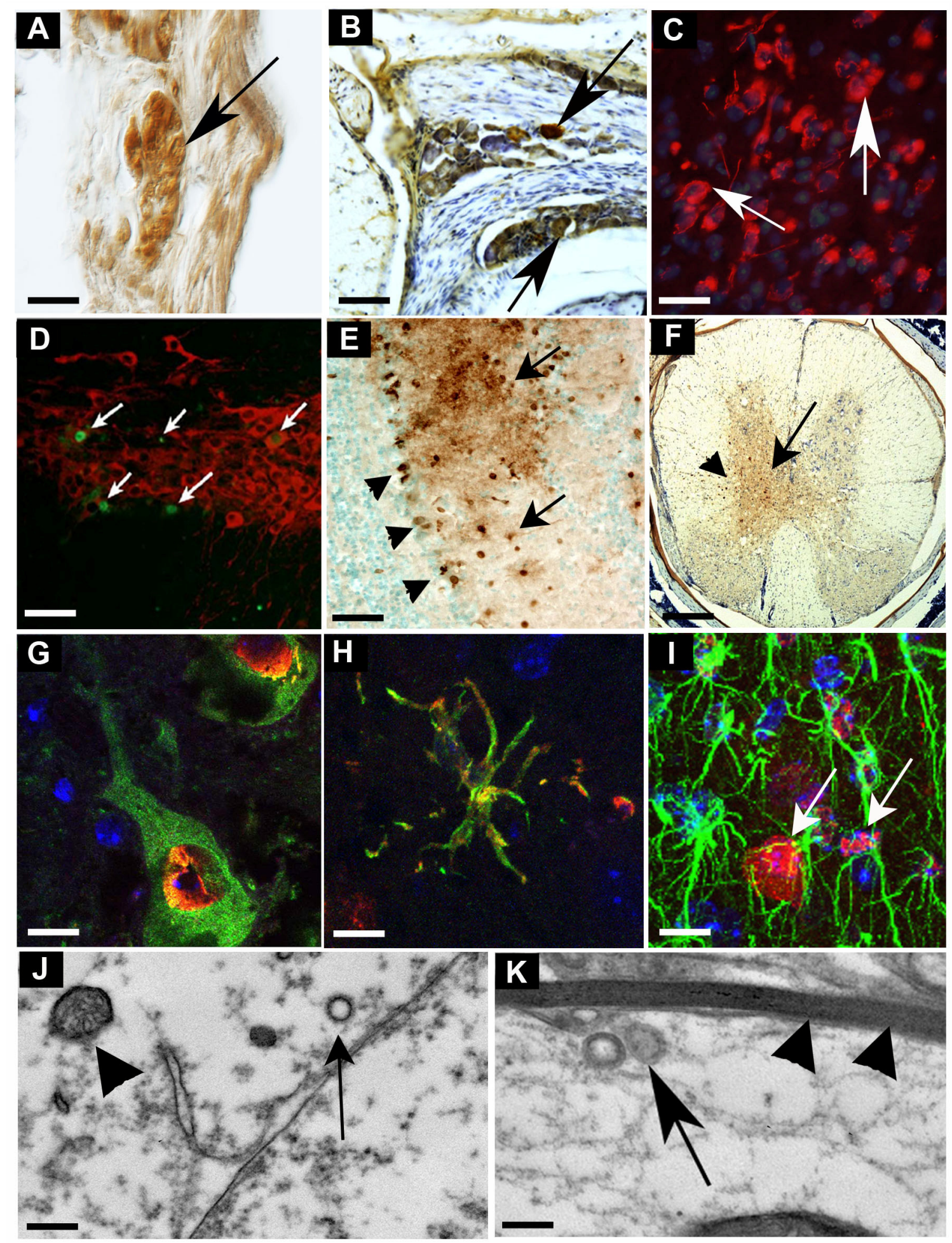
Figure 2.3 Localization of H5N1 Influenza Virus in Lung, Enteric Nervous System, Olfactory Bulb, and Brainstem

H5N1 virus is detected in lung starting at 3 days post infection (dpi). (A), At day 7 dpi, the virus is detected in bronchi (black arrows) as well as around the alveolar sacs (red arrows). An enlargement of the box marked in (A) is seen in (B). (C), Virus is not detected in bronchi or alveoli of age matched control mice. (D), An, enlargement of box in (C). (E), At day 3 dpi, H5N1 virus is detected in the neuronal ganglia of the enteric nervous system (arrow). (F), An enlargement of the box in (E). (G), Virus is not detected in enteric ganglia of control mice. (H), An enlargement of box in (G). (I), At day 7 dpi, foci of $\mathrm{H} 5 \mathrm{~N} 1$ virus are detected in all layers of the olfactory bulb. (J), An enlargement of box in (I). (I), H5N1 virus is detected in foci spanning the granule cell layer ( $\mathrm{gcl})$ and mitral cell layer $(\mathrm{mcl})$. $(\mathrm{K})$, Virus is not detected in the olfactory bulb of control mice. (L), An enlargement of box in (K). H5N1 virus is detected in the nucleus of the solitary tract starting at day 3 dpi (M), At day 7 dpi, the virus is detected in this nucleus as well as its adjacent dorsal motor nucleus of the vagus nerve. An enlargement of the box marked in $(\mathrm{M})$ is seen in $(\mathrm{N})$. (O), Virus is not detected in nucleus of the solitary tract or dorsal motor nucleus of the vagus nerve of control mice. $(\mathrm{P})$, An enlargement of box in $(\mathrm{O})$. (Q), At day 7 dpi, H5N1 virus is detected throughout many brainstem nuclei. (R), An enlargement of box in (Q). (S), Virus is not detected brainstem of control mice. (T), An enlargement of box in (S). Scale bars: A and C, $100 \mu \mathrm{m}$; B, D, E, G, J, L, N and P, 25 $\mathrm{F}$ and $\mathrm{H}, 10 \mu \mathrm{m} ; \mathrm{M}, \mathrm{O}, \mathrm{R}$ and $\mathrm{T}, 50 \mu \mathrm{m}$. 

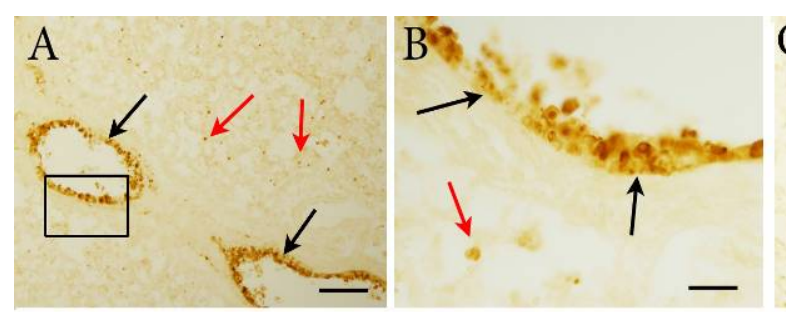

C

D

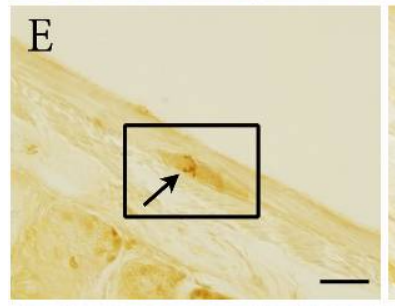

F

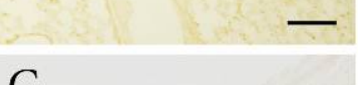

$\mathrm{H}$
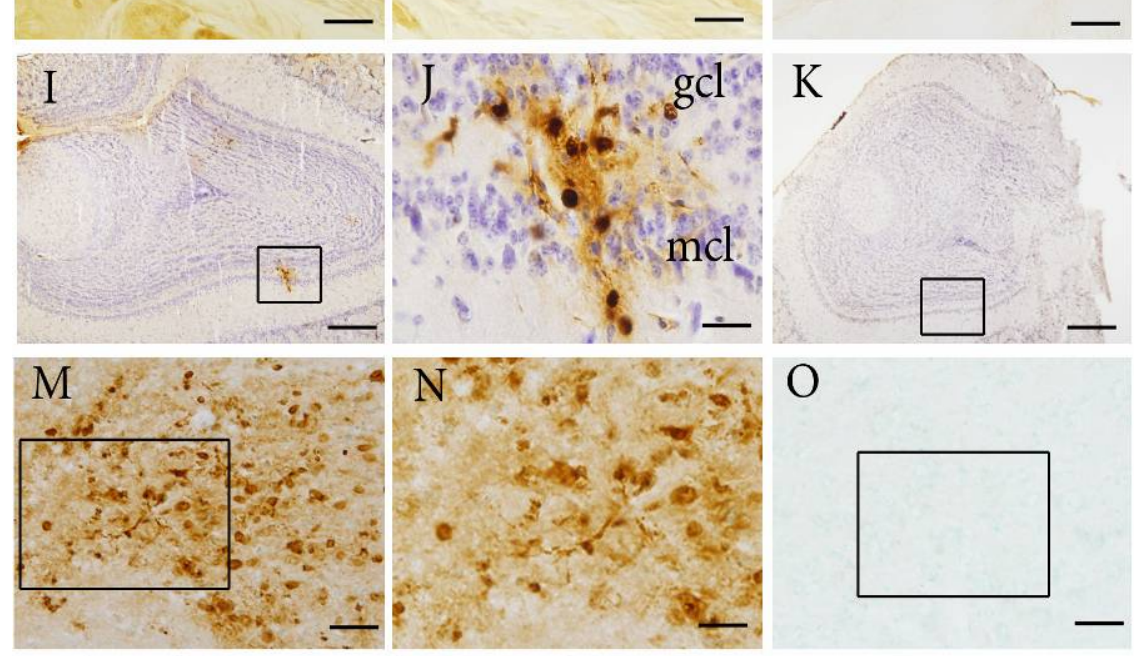

L $\quad$ -

Q

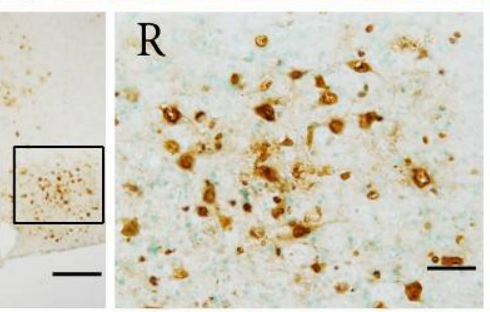

O

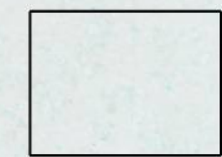
(e)

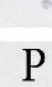

S

$\mathrm{T}$

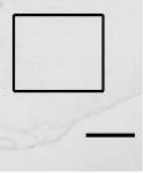


Figure 2.4 Localization of H5N1 Influenza Virus in Brainstem, Diencephalon, and Cortex

(A), At day 10 dpi, H5N1 is detected in locus coeruleus. (B), An enlargement of the box marked in (A). (C), H5N1 virus is not detected locus coeruleus of control animals. (D), Enlargement of box in (C). (E), At day 10 dpi, H5N1 is detected in the substantia nigra pars compacta (arrow). (F), An enlargement of the box in (E). (G), Virus is not detected in substantia nigra of control animals. $(\mathrm{H})$, An enlargement of box in (G). (I) At day 10 dpi, H5N1 is seen in focal regions of the diencephalon. This foci is observed in the ventrolateral thalamic nuclei. (J) An enlargement of box in I. (K) Virus is not detected in the thalamus of control animals. (L) An enlargement of box in K. (M) At day 10 dpi, $\mathrm{H} 5 \mathrm{~N} 1$ is detected in the foci of the cerebral cortex. One foci in the primary motor cortex is shown. An enlargement of the box marked in $(\mathrm{M})$ is seen in $(\mathrm{N})$. (O) Virus is not detected in cerebral cortex in control mice. (P) An enlargement of box in (O). Scale bars:

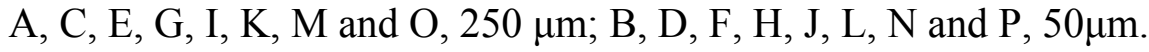



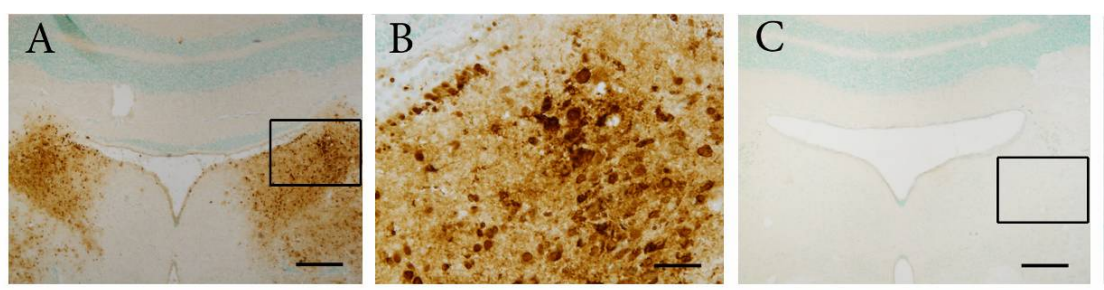

D
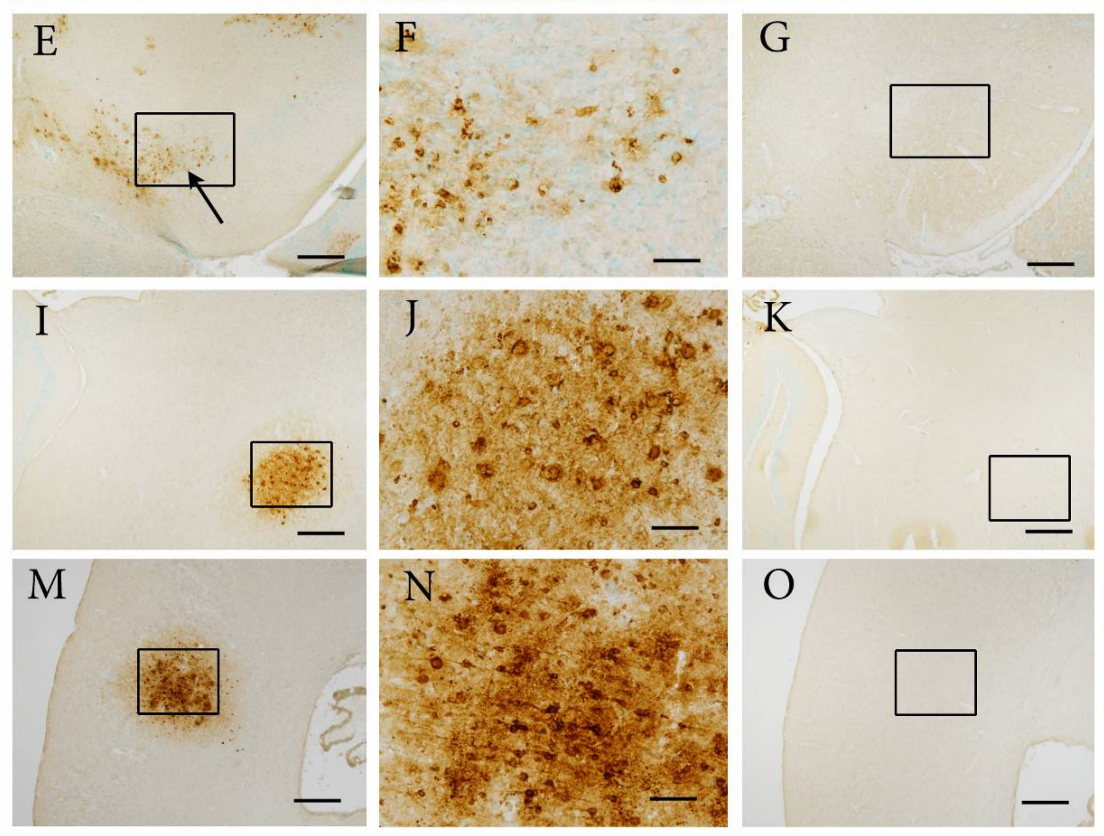

O
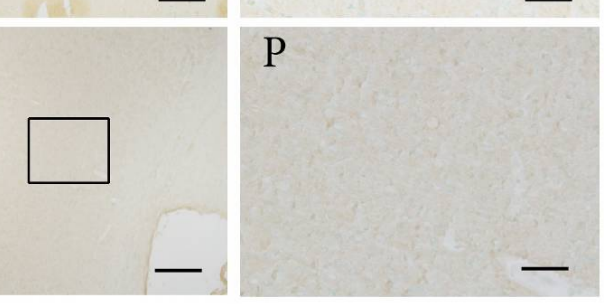

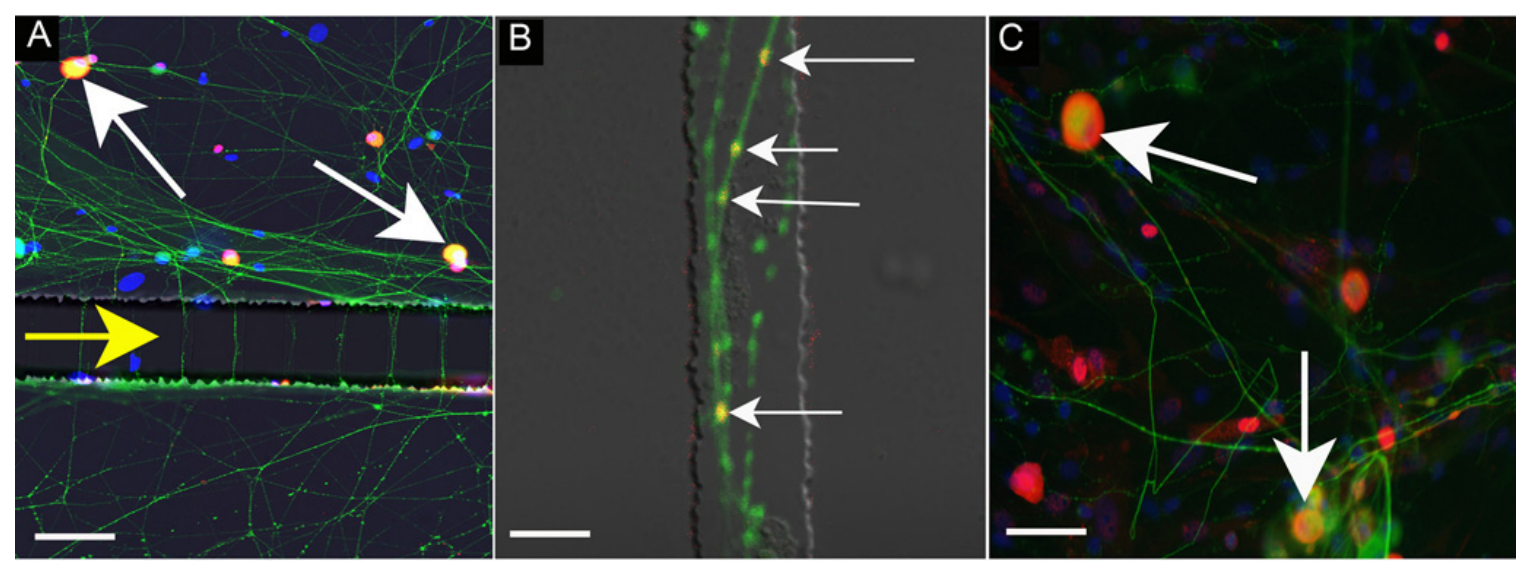

Figure 2.5 Transport of H5N1 Virus through Axons in Microfluidic Chambers

(A), A/Vietnam/1203/04 (H5N1) influenza virus $\left(2 \times 10^{6}\right.$ PFU suspended in $20 \mu \mathrm{L}$ of serum-free medium) was added to the process compartments of microfluidic chambers containing freshly dissociated dorsal root ganglia (DRG) (55). After 1 hour, the process compartment was washed twice by adding $130 \mu \mathrm{L}$ of serum-free medium to the top reservoir and collecting the flow-through in the bottom reservoir. $130 \mu \mathrm{L}$ of medium containing serum was then added to the top and bottom reservoirs of the process compartments to maintain hydrostatic balance. Twenty-four hours after addition of H5N1 virus to the process compartment (bottom), H5N1 NP is detected in neuronal bodies within the somal compartment (white arrows). Yellow arrow shows axons (green, beta3tubulin) traversing the series of $150-\mu \mathrm{m}$-wide grooves between the compartments. (B), High power photomicrograph of DRG axons (green, beta3-tubulin) growing though the $150-\mu \mathrm{m}$ grooves. Virus particles (arrows) within the axons appear yellow due to colocalization with neuronal beta3-tubulin. (C), Neuronal bodies (green) are infected after direct exposure to $\mathrm{H} 5 \mathrm{~N} 1$ virus (red, viral NP protein; arrows). Yellow indicates colocalization of proteins. Scale bars: A, $160 \mu \mathrm{m} ; \mathrm{B}, 6 \mu \mathrm{m} ; \mathrm{C}, 60 \mu \mathrm{m}$. 
virus can infect neurons following peripheral infection as well as by hematogenous spread directly infecting cells as would occur following its release from dying cells.

\subsubsection{Increased phosphorylation of alpha-synuclein protein in infected region}

One of the hallmarks of neurodegenerative disorders including Parkinson's and Alzheimer's disease is the aggregation of alpha-synuclein (107). This aggregation has been shown to be dependent on the phosphorylation of a number of serine residues in the synuclein protein (SYN), of which serine 129 appears to be most important (106). In 2-3 month old uninfected C57BL/6J mice, we observed pSer129SYN in few, if any, neurons at any level of the central neuraxis, including the dopaminergic neurons of the substantia nigra pars compacta (Fig. 2.6A). However, in H5N1 virus infected mice, we observed pSer129SYN-positive neurons at various levels of the neuroaxis including the brainstem and midbrain (Fig. 2.6B), hippocampus and cortex. The phosphorylation on Serine 129 residue appears to be directly related to $\mathrm{H} 5 \mathrm{~N} 1$ infection, as uninfected structures within the CNS did not show pSer129SYN upregulation. Using NIH Image, we quantified the expression of pSer129SYN in olfactory bulb, hippocampus, locus coeruleus, brainstem and cerebellum. Animals infected with H5N1 virus infection had significantly increased expression of both cellular and secreted pSer129SYN at all levels of the neuraxis examined except for cerebellum (Table 2.2). In addition to pSer129SYN expression, we also noted aggregated alpha-synuclein in hippocampus (Fig. 2.6C), cortex and brainstem, as seen in human proteinopathies such as Parkinson's and Alzheimer's diseases (107).

\subsubsection{Apoptosis and long-term activation of microglia}

To determine whether H5N1 virus infection of the CNS resulted in cell death, we examined markers of apoptotic and necrotic cell death (activated caspase 3 (275) and FluoroJade B staining (284), respectively). We also assessed dopaminergic neuron number in the substantia nigra pars compacta $(\mathrm{SNpc})$. In regions of the brain where A/VN/1203/04 virus had been detected, we found apoptotic (Fig. 2.6D) but not necrotic cells. We also noted activated microglia (Fig. 2.6E), which indicate an inflammatory process (285), in these regions. Microgliosis persisted in the CNS after the acute infection resolved and was observed during the entire 90-day study period (Fig. 2.6F). This prolonged inflammatory response is similar to that described in humans with idiopathic Parkinson's disease (286) and with parkinsonism induced by the neurotoxin MPTP (131).

\subsubsection{A reduction in the number of tyrosine hydroxylase (TH) positive dopaminergic neuron in the $S N p c$}

Cell counts of TH-positive dopaminergic neurons in the SNpc 60 days following $\mathrm{H} 5 \mathrm{~N} 1$ virus infection demonstrated a $17 \%$ reduction of TH-positive dopaminergic neurons $(8963 \pm 384$ in control and $7481 \pm 453$ in H5N1 virus infected mice, $n=4, p \leq$ 016). 

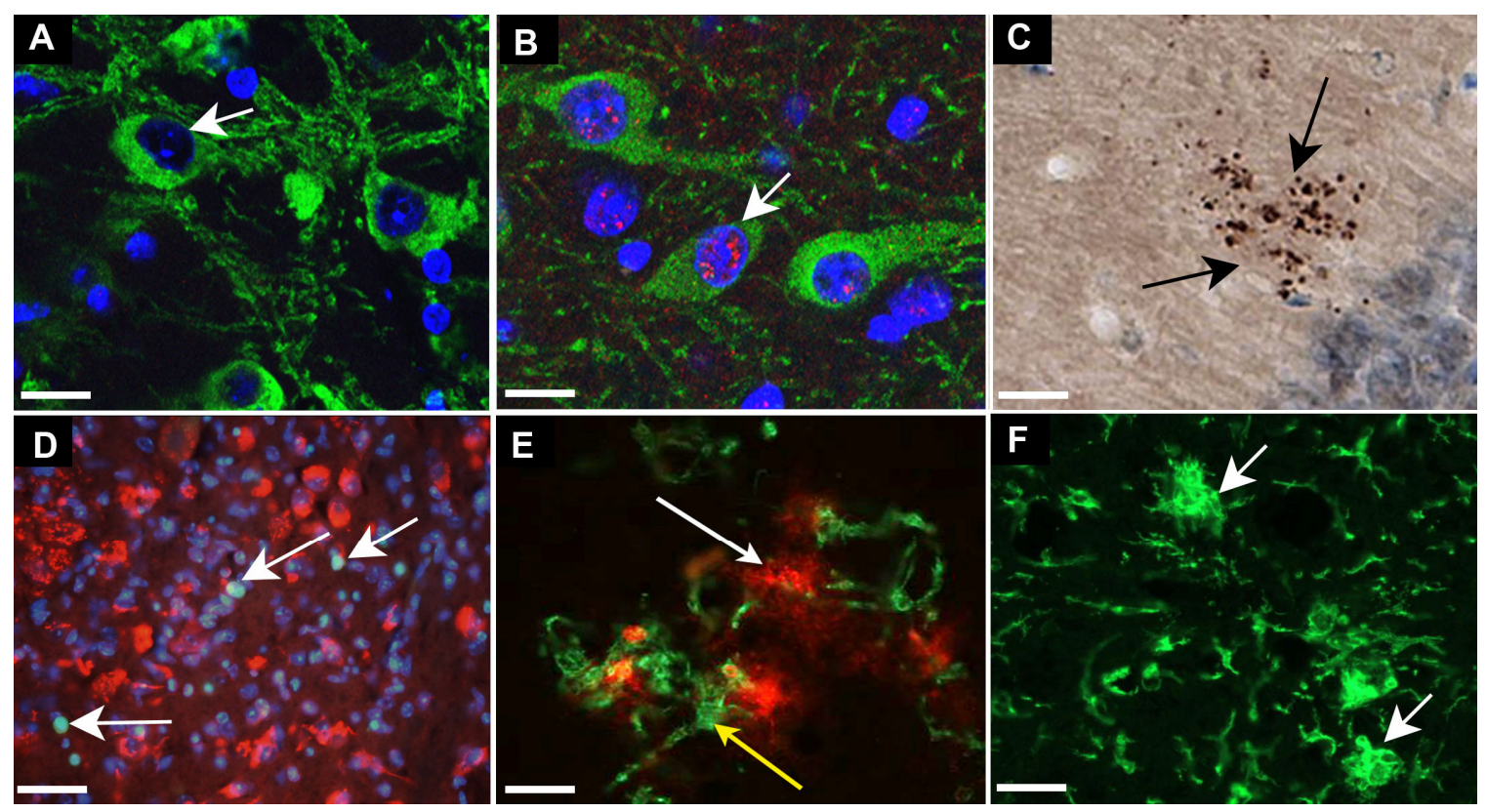

Figure 2.6 H5N1 Influenza Virus Induces a Parkinsonian Phenotype

(A), In uninfected C57BL/6J mice, no pSer129SYN is seen in dopaminergic neurons of the SNpc (green). (B), At day 60 dpi, pSer129SYN (white arrows) is seen in the nuclei of dopaminergic neurons (green) in the SNpc. (blue, DAPI nuclear stain.) (C), At day 90 dpi, aggregated alpha synuclein (arrows) is seen in the hippocampus. (D), At day $10 \mathrm{dpi}$, activated caspase-3 (arrows) is seen in brainstem regions that contain NP protein (red). The cells expressing activated caspase-3 do not appear to be NP-positive. (E), At day 10 dpi, activated microglia marked by anti-Iba-1 (green; yellow arrow) surround infected cells (red; white arrow). (F), At day 60 dpi, activated microglia (arrows) persist in midbrain regions and all other regions previously infected by H5N1. Findings were similar at day 90 dpi Scale bars: A and B, $30 \mu \mathrm{m}$; C, $25 \mu \mathrm{m}$; D, $40 \mu \mathrm{m}$; E, $35 \mu \mathrm{m}$; F, 50 $\mu \mathrm{m}$. 
Table 2.2 Relative Density Measurements of pSer129SYN Expression in Brain

\begin{tabular}{|c|c|c|c|c|c|c|}
\hline \multirow{2}{*}{ Structure } & \multicolumn{3}{|c|}{ Proteinase-K, non treated } & \multicolumn{3}{|c|}{ Proteinase-K, treated } \\
\hline & Control & H5N1 & Statistics & Control & H5N1 & Statistics \\
\hline Olfactory bulb & $100 \pm 19$ & $178 \pm 27$ & $\mathrm{p} \leq 0.05$ & $100 \pm 19$ & $189 \pm 23$ & $\mathrm{p} \leq 0.01$ \\
\hline Hippocampus & $100 \pm 21$ & $277 \pm 21$ & $\mathrm{p} \leq 0.001$ & $100 \pm 21$ & $244 \pm 25$ & $\mathrm{p} \leq 0.001$ \\
\hline Locus coeruleus & $100 \pm 20$ & $120 \pm 12$ & n.s. & $100 \pm 7$ & $168 \pm 10$ & $\mathrm{p} \leq 0.001$ \\
\hline Solitary nucleus & $100 \pm 18$ & $190 \pm 13$ & $\mathrm{p} \leq 0.001$ & $100 \pm 7$ & $163 \pm 8$ & $\mathrm{p} \leq 0.001$ \\
\hline Lower brainstem & $100 \pm 17$ & $274 \pm 37$ & $\mathrm{p} \leq 0.001$ & $100 \pm 21$ & $135 \pm 14$ & n.s. \\
\hline Cerebellum & $100 \pm 8$ & $100 \pm 3$ & n.s. & $100 \pm 16$ & $108 \pm 12$ & n.s. \\
\hline
\end{tabular}

The brain areas were stained with anti-pSer129SYN antibody by immunohistochemistry. Both soluble pSer129SYN (Proteinase-K, non treated) and insoluble (Proteinase-K, treated) forms of this phosphoprotein were determined. Statistical analysis was performed using GraphPad software (error bars, s.e.m.). 


\subsection{Discussion}

Reports of influenza-associated neurological syndromes are found as far back as 1385 and have continued through more recent influenza outbreaks (5). There is a substantial amount of evidence that influenza can directly lead to encephalitis $(5,28-33)$, although the link with development of neurodegenerative diseases including Parkinson's disease are controversial. Much of the linkage of parkinsonism with influenza are based on the postencephalic parkinsonism that followed an outbreak of von Economo's encephalopathy (EL) subsequent to the 1918 pandemic influenza outbreak (213). This includes epidemiological data $(2,3)$ and physical findings of type A influenza antigens in EL patients (36). Evidence against the role of influenza as a parkinsonian agent include the lack of viral RNA recovered from brains of postencephalic parkinsonian patients (223), the absence of any known mutations that would make the 1918 species of H1N1 virus neurotropic (213), and questions regarding the timeline of pandemic flu and EL (42). Recently, Kobasa et al administered the 1918 H1N1 influenza virus that was generated by plasmid-based reverse genetics and found no evidence of direct neurotropism (38). However, this engineered virus did induce a robust induction of cytokines including monocyte chemotactic protein-1 (MCP-1), macrophage inflammatory protein-1 $\beta$ (MIP-1 $\beta$ ), monocyte chemotactic protein-2 (MIP-2), macrophage inflammatory protein-3 $\alpha$ (MIP-3 $\alpha$ ), IL-1 $\beta$, IL-6, IL-12 (p40), IL-18 and granulocytecolony stimulating factor (G-CSF) (39), which can be secondarily activated in the brain without direct infection (40). Several of these cytokines have been implicated in the pathophysiology of Parkinson's disease (41).

In many cases of encephalitis, as well as toxin-induced parkinsonism, the offending agent may cause a long lasting immune response in the brain that persists many years after the insult has resolved (131), leading to a "hit and run" mechanism where the original insult is no longer present but the secondary sequelae persists (287). This would fit our observations that $\mathrm{H} 5 \mathrm{~N} 1$ virus is not detectable in the brain after 21dpi, but there is a long-lasting activation of microglia and a significant decrease through 60 days of THpositive dopaminergic neurons in SNpc. Thus, if one accepts that influenza can activate the innate CNS immune system $(43,44)$ and may induce a loss of neurons, there would be the necessity of a second hit that would lead to development of additional neuronal loss passing the threshold necessary for induction of parkinsonian symptoms (288) that would not have occurred without the priming that occurs via the influenza infection (46).

In conclusion, we find that the highly infectious, neurotropic A/VN/1203/04 (H5N1) virus progresses from the peripheral nervous system into the CNS, where it activates the innate immune response in the brain. We have also shown for the first time that $\mathrm{H} 5 \mathrm{~N} 1$ influenza infection of the CNS can induce not only parkinsonian symptoms but also a significant increase in phosphorylation and aggregation of alpha synuclein, which likely results in the observed SNpc dopaminergic neuron degeneration (47). Thus, we suggest that any pandemic influenza that activates the immune system in brain could contribute to CNS disorders of protein aggregation $(20,48)$ and viruses may be an important etiological agent in the developmental sequelae of neurodegenerative diseases including Parkinson's disease. 


\section{CHAPTER 3. INFLAMMATORY EFFECTS OF HIGHLY PATHOGENIC H5N1 INFLUENZA VIRAL INFECTION IN THE CENTRAL NERVOUS SYSTEM}

\subsection{Introduction}

Viral infection is one mechanism that has been implicated in initiation and progression of parkinsonism. The etiology of Parkinson's disease is multivariate, ranging from identified genetic mutations to strict environmental causation (289). There is a significant literature that documents parkinsonism following exposure to viruses (5), including the influenza virus (42). While most influenza infections in humans result in upper respiratory tract infections, occasionally the brain is also be affected. In fact, involvement of the CNS during influenza infections can be fatal, particularly in young patients (290).

Experimental evidence has shown that type A influenza viruses are neurotropic, i.e. they can travel into the nervous system following systemic infection $(180,221,222)$, a finding confirmed with the H5N1 influenza virus $(256,270)$. Once in the CNS, H5N1 (A/VN/1203/04) infection can initiate a parkinsonian pathology that includes bradykinesia, a decreased in the number TH-positive dopaminergic neurons in SNpc, increased levels of alpha-synuclein phosphorylation and aggregation, and activation of microglia; each of which persisted at least 60 days after resolution of the infection (270). To determine if the pathology worsened with age, we examined: 1) SNpc tyrosine hydroxylase-positive dopaminergic neuron number and striatal dopamine and its metabolites contents through 90 days post infection, 2) the inflammatory effect of infection by quantitatively measuring the total number of resting and activated microglia in the SNpc, and 3) the production of cytokines in regions of the brain infected by H5N1 influenza virus. We found that infection with $\mathrm{H} 5 \mathrm{~N} 1$ influenza virus induces a significant, but transient reduction of both TH-positive dopaminergic neurons in the SNpc and dopamine (and its metabolites) in the striatum. Examination of other indolamines demonstrated a significant and sustained reduction of serotonin in regions of the brain infected with $\mathrm{H} 5 \mathrm{~N} 1$ influenza virus. We also observed that areas of the brain infected with H5N1 influenza virus expressed increased levels of proinflammatory cytokines, chemokines, and growth factors.

\subsection{Materials and Methods}

All experiment using the highly pathogenic H5N1 influenza virus (A/VN/1203/04) were conducted in a biosafety level 3+ laboratory approved for use by the U.S. Department of Agriculture and the Centers for Disease Control. This facility is authorized for the exclusive use of the Division of Virology and other approved scientists at St Jude Children's Research Hospital. 


\subsubsection{Virus stock preparation and inoculation of mice with $\mathrm{H} 5 \mathrm{~N} 1$}

Stock viruses were prepared by propagating neurotropic A/VN/1203/04 (H5N1) allantoic cavity of 10-days-old embryonated chicken eggs for 40 to 48 hours at $37^{\circ} \mathrm{C}$. Virus stock was aliquoted then stored at $-70^{\circ} \mathrm{C}$ until use. Viral infectious titers were determined using the method of Reed and Muench (271), and expressed in log 10 of the $50 \%$ egg infectious dose per $1.0 \mathrm{ml}$ of fluid $\left(\mathrm{EID}_{50} / \mathrm{mL}\right) .6-8$ week old C57BL/6J female mice (Jackson Labs, Bar Harbor, ME) were anesthetized by isofluorane inhalation and infected intranasally with $30 \mu \mathrm{L}$ of allantoic fluid diluted in PBS to the target virus infectious titer $\left(10^{2} \mathrm{EID}_{50}\right)$. One group of animal received $0.9 \%$ saline was used as agematched control.

\subsubsection{Immunocytochemistry}

Mice were deeply anesthetized with Avertin and transcardially perfused with $0.9 \%$ saline followed by $10 \%$ neutral buffered formalin at 10,21, 60 and 90 days post infection. Brains were removed and postfixed for 3 weeks in $10 \%$ neutral buffered formalin to ensure that the virus particles present in the tissue have been killed. Brains were cryoprotected with $30 \%$ sucrose in PBS, serially sectioned in the coronal plane at 40 micrometers and placed in PBS filled 24 well plates. Free floating sections were immunolabeled with antibodies directed against tyrosine hydroxylase (TH) (rabbit, P40101-0, PelFreeze, Rogers, AK, 1:500) (to identify dopaminergic neurons) and ionized calcium binding adapter protein-1 (Iba-1) (rabbit, 019-19741, Wako Chemicals, Richmond, VA, 1:500) (to identify microglia) overnight. Primary antibodies were visualized using the ABC method (PK-6102,Vector Laboratories, Burlingame, CA) with chromogens, 3,3-diaminobenzidine (SK-4100 Vector Laboratories, Burlingame, CA) (for DA neurons) and the Vector VIP substrate (SK-4600 Vector Laboratories, Burlingame, CA) (for microglia) respectively. Sections were mounted on Superfrost-Plus (12-550, Fisher, Pittsburgh, PA) slide and counter stained with neutral red to visualize Nissl substance, and then dehydrated, cleared and mounted with Permount (SP15-500, Sigma, St. Louis, MO).

\subsubsection{Stereological cell count using the optical fractionator methods}

The total number of TH-positive neurons in both hemispheres of SNpc was estimated using the optical fractionator method (279) from StereoInvestigator (version 7.0; MicroBrightField, Colchester, VT). The outline of the SNpc in both hemispheres was delineated at low power ( $4 \times$ magnification). An unbiased counting frame $(60 \times 60 \mu \mathrm{m})$ was placed at the intersections of a grid (frame size $200 \times 200 \mu \mathrm{m}$ ) randomly superimposed on a video image of the contoured sections. Sections were examined under a high power $(100 \times$ objective lens, numerical aperture $(\mathrm{NA})=1.3)$ on a $\mathrm{BX} 51$ microscope (Olympus, Center Valley, PA) with a MAC5000 motorized XYZ axis computercontrolled stage and a CX9000 CCD video camera (MicroBrightField, Colchester, VT). 
TH-positive DA neurons were counted at the depth at which their nucleus was focused in each counting area. The reliability of the estimates was measured by calculation of coefficient of error (279). Gundersen coefficients of error for $m=1$ was all less than or equal to 0.10 . Statistical significance was calculated using a one-way ANOVA followed by Student-Newman-Keuls post hoc test (280). The longest length of neuronal cell body was measured using the Neurolucida program (version 7.0, MicroBrightField, Colchester, VT). Raw data was converted to percent of control.

Based on the shape and morphology (291), the number of the activated and the resting microglia in both hemispheres of the SNpc were estimated using the same methods described above.

\subsubsection{Biochemical measurement of monoamine neurotransmitters}

At 10, 21, 60 and 90 days post infection, C57BL/6J mice were deeply anesthetized with Avertin and transcardially perfused with $0.9 \%$ saline to remove the majority of the blood from the brain vasculature. Brains were rapidly removed and placed in a brain matrix (BS-AL-5000C, Braintree Scientific, Braintree, MA) and sliced into 1 $\mathrm{mm}$ thick sections and placed on an ice-cooled plate. Tissues were dissected from the SN (Bregma: -2.70 to -3.70 ), striatum (Bregma: +0.14 to $+1.26 \mathrm{~mm}$ ), brainstem (Bregma: 5.40 to $-6.70 \mathrm{~mm}$ ), cortex (Bregma: -1.70 to $-2.70 \mathrm{~mm}$ ), and hippocampus (Bregma: -1.70 to $-2.70 \mathrm{~mm}$ ) (273). Tissues were homogenized in a chilled $0.3 \mathrm{M}$ perchloric acid and centrifuged for 15 minutes at $10,000 \mathrm{~g}$ at $4^{\circ} \mathrm{C}$. The supernatants were saved and stored at $70^{\circ} \mathrm{C}$ until used. A variety of monoamine transmitters; dopamine (DA), and its metabolites 3,4-dihydroxyphenylacetic acid (DOPAC), and homovanillic acid (HVA); norepinephrine (NE); 5-hydroxytryptamine (5-HT) and its metabolites 5hydroxyindoleacetic acid (5-HIAA) were analyzed using reverse-phase ion pairing HPLC, combined with electrochemical detection (EC) detection under isocratic elution conditions. The amount of monoamine neurotransmitters in the tissues were determined by injecting known concentration of monoamine neurotransmitters and extrapolating from a standard curve. Statistical difference was determined using a one-way ANOVA followed by Student-Newman-Keuls post hoc test (292).

\subsubsection{Quantification of cytokines, chemokines and growth factors}

The concentration of interleukin (IL)-1 $\alpha$, IL-1 $\beta$, IL-2, IL-6, IL-9, IL-10, IL12(p70), IL-13, tumor necrosis factor alpha (TNF- $\alpha$ ), interferon gamma (IFN- $\gamma$ ), granulocyte-macrophage colony-stimulating factor (GM-CSF), granulocyte colonystimulating factor (G-CSF), macrophage colony-stimulating factor (M-CSF), eotaxin, interferon-inducible protein 10 (IP-10), cytokine-induced neutrophil chemoattractant $(\mathrm{KC})$, monocyte chemotactic protein-1 (MCP-1), macrophage inflammatory proteins (MIP)-1 $\alpha$, MIP-1 $\beta$ and vascular endothelial growth factor (VEGF) proteins was simultaneously analyzed from dissected brain regions and lung, using the Luminex 200 
system (Luminex Corp., Austin, TX) and the Milliplex mouse cytokine kit (MPXMCYTO-70K-20, Millipore, Billerica, MA).

At 3, 10, 21, 60 and 90 days post inoculation, dissected tissues were homogenized in a buffer containing $50 \mathrm{mM}$ Tris- $\mathrm{HCl}$ (pH 7.4), 2.5mM EDTA, 0.1\% Triton X-100, $150 \mathrm{mM} \mathrm{NaCl}$ with a protease and phosphatase inhibitor cocktail (Complete mini, PhosphoStop, Roche, Indianapolis, IN). The tissues lysates were then incubated for 30 minutes on ice and centrifuged at $12,000 \mathrm{~g}$ for 15 minutes. Supernatants were aliquoted and stored at $-70^{\circ} \mathrm{C}$ until used. To quantify cytokine levels, the supernatants were incubated with the microspheres coated with capture antibodies for each analyte for 2 hours. After rinsing, biotinylated detection antibodies were added into each well and incubated for 1 hour. Streptavidin-phycoerythrin conjugates were added and fluorescence intensity was measured by the Luminex 200 reader (Luminex Corp., Austin, TX). Concentration of each cytokine was calculated from a 5-parameter logistic curve obtained from the known concentration of cytokines, using MILLIPLEX Analyst software (Millipore, Billerica, MA, USA). All results were normalized with total protein concentration measured from each tissue lysate ( $\mathrm{pg} / 25 \mu \mathrm{g}$ of total protein).

\subsection{Results}

\subsubsection{Transient loss of TH-positive phenotype in dopaminergic neurons following H5N1 infection}

To determine if $\mathrm{H} 5 \mathrm{~N} 1$ infection can directly induce parkinsonism by damaging dopaminergic neurons, we stereologically assessed the number of TH-positive dopaminergic neurons in the SNpc (Fig 3.1A) and empirically determined the amount of total DA, HVA, and DOPAC in the SN, cortex, brainstem, and hippocampus (Fig 3.2A).

A dramatic decrease in the number of TH-positive neurons in the SNpc was observed at the peak of H5N1 infection from 10 to 21 dpi where we measured approximately $60 \%$ reduction in the number of TH-positive neurons, compared to noninfected control mice. However, from 21 to $60 \mathrm{dpi}$, we saw a recovery in neuron number which is $20 \%$ less than that of control mice. By 90 dpi, we saw no difference in THpositive SNpc DA neuron number (Fig 3.1A). At 10 and 60 dpi, TH-positive DA neurons appeared shrunken. The longest length of TH-positive neuronal cell bodies was reduced by 10 to $20 \%$, however the size of these cells recovered and appeared similar to that seen in the non-infected control mice at day 90 dpi (Fig 3.1B). Examination of cells in the SNpc showed no evidence of cell division using both morphological and immunohistochemical methods. Thus, there is no compelling evidence that DA neurons in the SNpc underwent any form of cell division. It is most likely that active H5N1 infection causes SNpc DA neurons to transiently reduce their metabolic capacity and cell size, each leading to a loss of the dopaminergic phenotype in neurons. 

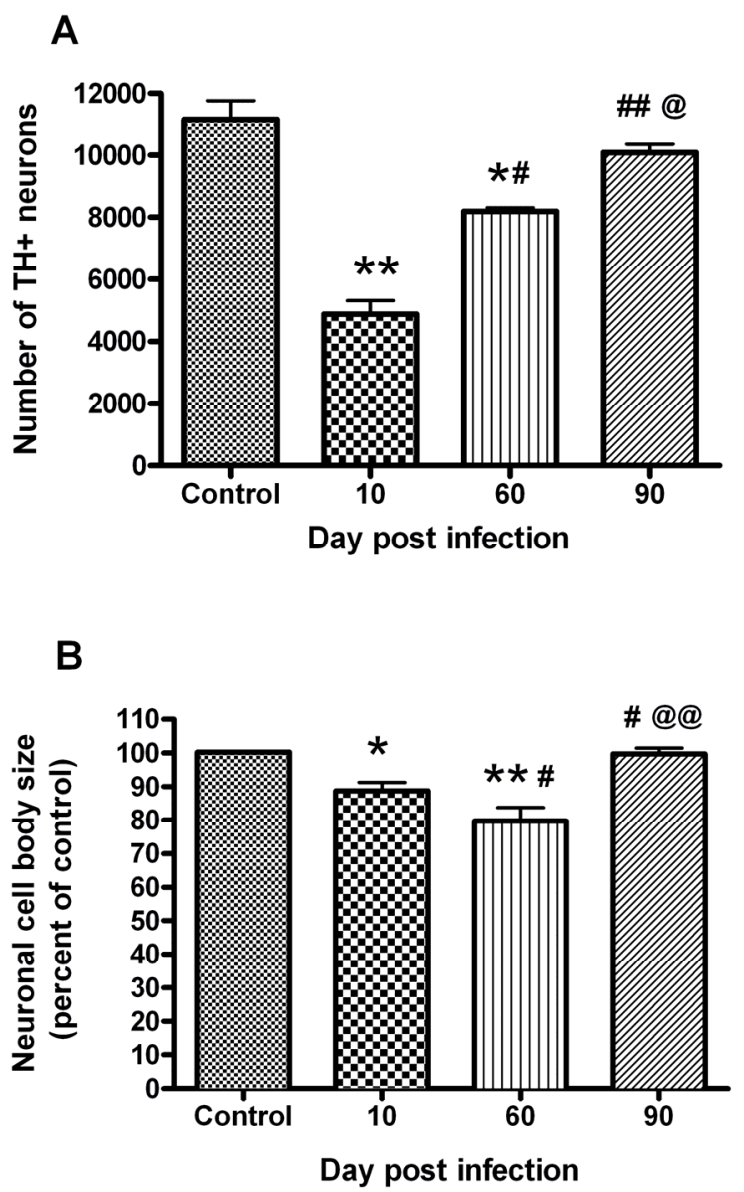

Figure 3.1 The Size and Number of TH-Positive Dopaminergic Neurons

(A), Following H5N1 infection, the number of TH-positive dopaminergic neurons in the SNpc was reduced approximately $60 \%$ at 10 dpi and $20 \%$ at 60 dpi from the non-infected control mice $(11170 \pm 599 \mathrm{TH}$-positive DA neurons in control, $4882 \pm 429 \mathrm{TH}$-positive DA neurons in H5N1-infected mice at $10 \mathrm{dpi}, \mathrm{n}=4, \mathrm{P}<0.001 ; 8195 \pm 123$ TH-positive DA neurons in H5N1-infected mice 60 dpi, n=4, P $<0.01$ ). At 90 dpi, the number of THpositive dopaminergic neurons in the SNpc was similar to that of control mice $(10090 \pm$ 259 TH-positive DA neurons in H5N1-infected mice 90 dpi, n=4, n.s.). The atrophic shape of TH-positive neurons was frequently detected in the SN of infected mice. (B), The longest length of TH-positive neuronal cell bodies was reduced by 10 to $20 \%$, however the size of these cells recovered and appeared similar to that seen in the control mice at 90 dpi. Statistical significance was determined by one-way ANOVA followed by Student-Newman-Keuls test. (*: comparison with control mice; ${ }^{*}$ : comparison with $10 \mathrm{dpi}$ mice; ${ }^{@}$ : comparison with 60 dpi mice; *, ${ }^{*}$ and ${ }^{@}: \mathrm{p}<0.05 ; * *{ }^{\#}$ and ${ }^{@}$ : $\left.\mathrm{P}<0.001\right)$. 

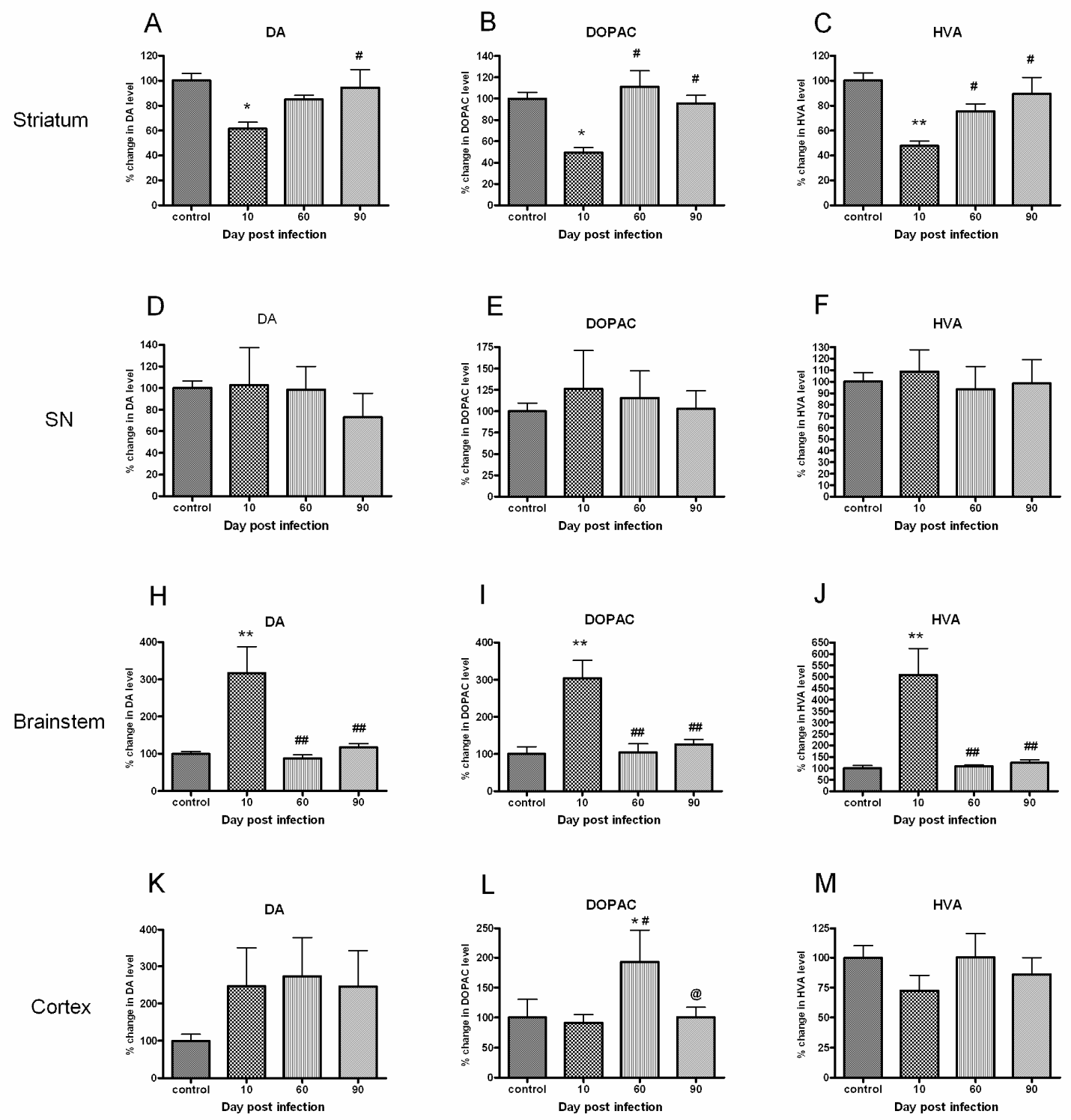

Figure 3.2 Percent Change in the Amount of DA, DOPAC, and HVA in Brain Following H5N1 Infection

(A), At 10 dpi, the amount of striatal DA was significantly decreased, compared to DA level of the non-infected control mice. At 90 dpi, DA levels were significantly increased from DA levels of 10 dpi mice and recovered to baseline levels. This pattern of a transient decrease at 10 dpi followed by recovery at 60 to 90 dpi was also seen in levels of HVA and DOPAC in striatum (B-C). In the brainstem, DA, DOPAC, and HVA levels were sharply increased at $10 \mathrm{dpi}$, then returned to their basal levels (G-I). Statistical significance was determined by one-way ANOVA followed by Student-Newman-Keuls test $\left(*\right.$ : comparison with control mice; ${ }^{\sharp}$ : comparison with 10 dpi mice; ${ }^{\circledR}$ : comparison with 60 dpi mice; * , ${ }^{\circledR}$ and ${ }^{@}: \mathrm{p}<0.05 ; * *{ }^{\# \#}$ and ${ }^{@} @$ : $\left.\mathrm{P}<0.001\right)$. 


\subsubsection{Transient reduction of dopamine in striatum following $\mathrm{H} 5 \mathrm{N1}$ infection}

We used reverse phase HPLC with electrochemical detection to determine if infection with $\mathrm{H} 5 \mathrm{~N} 1$ affects the level of dopamine and its metabolites in the major target of DA neurons in the SNpc, the striatum. At $10 \mathrm{dpi}$, the amount of striatal DA was significantly decreased by approximately $40 \%$ from that of non-infected control mice. The levels of DA then increased between 60 and 90 dpi at which time it recovered to baseline levels (Fig 3.2A). A similar pattern was also seen in the levels of HVA and DOPAC (Fig 3.2B-C). We also compared the turnover ratio of DA in the striatum $((\mathrm{DOPAC}+\mathrm{HVA}) / \mathrm{DA})$ to determine if infection with H5N1 altered DA metabolism. Despite alterations in levels of DA, its turnover was unchanged.

\subsubsection{Effect of H5N1 infection on NE and 5-HT levels in the CNS}

Reverse phase HPLC with electrochemical detection was used to determine if infection with $\mathrm{H} 5 \mathrm{~N} 1$ affected levels of NE, 5-HT, and 5-HIAA in the SN, striatum, brainstem, cortex, and hippocampus. No significant difference was found in the level of $\mathrm{NE}$ in the striatum, $\mathrm{SN}$, and brainstem (Fig 3.3A-C). NE level was transiently increased approximately $50 \%$ in the hippocampus at $60 \mathrm{dpi}$ (Fig 3.3D) and decreased by $70 \%$ in the cortex at 10 dpi (Fig 3.3E).

In the striatum and $\mathrm{SN}$, the levels 5-HT were significantly decreased approximately $50 \%$ from the non-infected control mice at $10 \mathrm{dpi}$ and remained low through 90 dpi (Fig 3.4A, C). A similar reduction was seen in 5-HIAA levels (Fig 3.4B, D). In cortex, 5-HT was reduced by $85 \%$ at $10 \mathrm{dpi}$ and remained reduced through $90 \mathrm{dpi}$ (Fig 3.4I). A smaller reduction (70\%) was seen in 5-HIAA levels at day $10 \mathrm{dpi}$ (Fig 3.4J) that increased slightly to $60 \%$ of control levels through $90 \mathrm{dpi}$.

\subsubsection{H5N1 infection increases the number of microglia in the SNpc}

Microglia are the resident immune cells of the CNS (293). In surveillance mode, they are said to be resting and have a characteristic histological appearance with long slender tendrils radiating from a cell body (Fig 3.5A). Once exposed to infection, injury or trauma (294) they undergo a transformation in which they retract and thicken their processes, assuming a more amoeboid morphology (295) (Fig 3.5B). To determine if exposure to $\mathrm{H} 5 \mathrm{~N} 1$ alters the morphology and number of microglia in the $\mathrm{SNpc}$, we used the optical fractionator method to assess resting and activated microglia. Non-infected C57BL/6J mice had approximately 7500 total microglia in the SNpc. Approximately $10 \%$ were structurally classified as activated, while $90 \%$ were classified as resting. Sixty days after intranasal inoculation with $\mathrm{H} 5 \mathrm{~N} 1$, we found a $67 \%$ increase in total microglia number in the SNpc. Examination of microglia subtype revealed a 300\% increase in activated microglia and a $33 \%$ increase in resting microglia. The increase in total microglia, as well as percent increase in activated microglia was unchanged from 60 to 90 dpi (Fig 3.5C), suggesting a long-term -if not permanent- increase in these cells. 

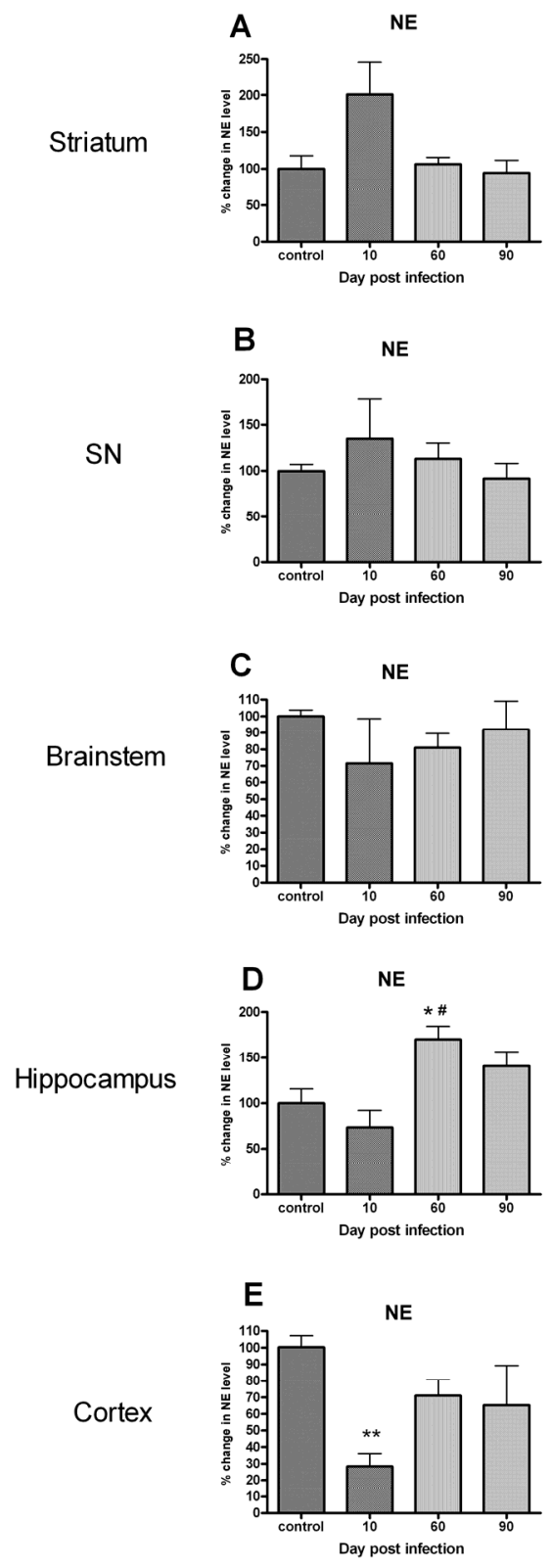

Figure 3.3 Percent Change in the Amount of NE in Brain Following H5N1 Infection

(A-C), No significant difference was found in the level of NE in the striatum, SN, and brainstem. (D), NE level was transiently increased in the hippocampus at $60 \mathrm{dpi}$, compared to the level of control and the $10 \mathrm{dpi}$ mice, and returned to basal levels at 90 dpi. (E), In cortex, we observed a transient $72 \%$ reduction of NE that ebbed at day 10 dpi and rebounded to basal levels through $90 \mathrm{dpi}$. Statistical significance was determined by one-way ANOVA followed by Student-Newman-Keuls test (*: comparison with control mice; ${ }^{\#}$ : comparison with 10 dpi mice; ${ }^{\circledR}$ : comparison with 60 dpi mice; *, ${ }^{*}$ and ${ }^{\circledR}$ : $\mathrm{p}<0.05 ; * *,{ }^{\#}$ and ${ }^{@}$ : $\mathrm{P}<0.001$; error bars, s.e.m.). 

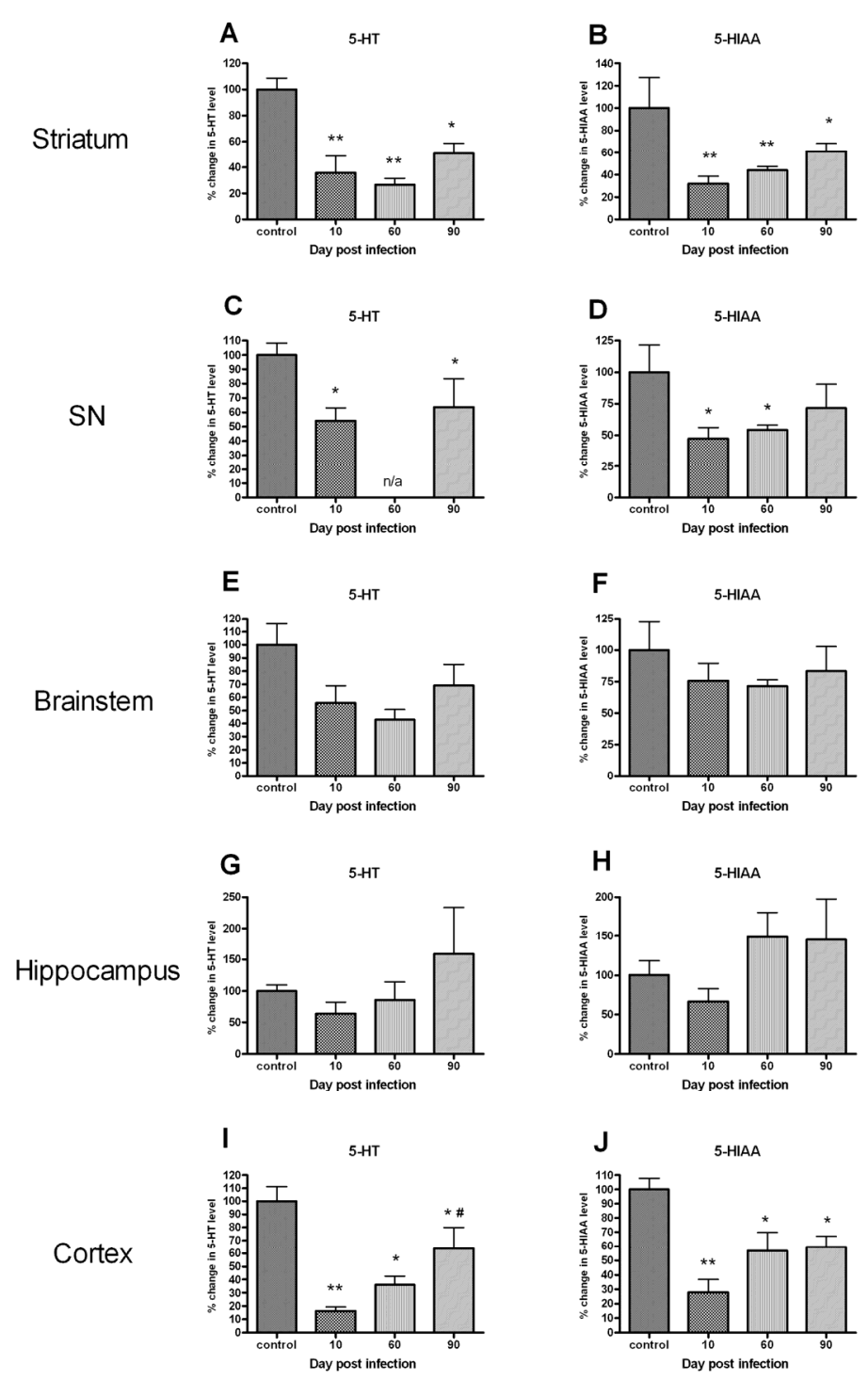

Figure 3.4 Percent Change in the Amount of 5-HT and 5-HIAA in Brain Following H5N1 Infection

(A), (C) and (I), The level of 5-HT was significantly reduced in the striatum, SN, and cortex at 10 dpi and remained low through 90 dpi. (B), (D) and (J), A similar reduction pattern was seen in the level of 5-HIAA. (E-H), Although the mean levels of 5-HT and 5HIAA in hippocampus and brainstem trended lower, none of these changes achieved statistical significance. Statistical significance was determined by one-way ANOVA followed by Student-Newman-Keuls test $\left(*\right.$ : comparison with control mice; ${ }^{\#}$ : comparison with 10 dpi mice; ${ }^{\circledR}$ : comparison with 60 dpi mice; $*,{ }^{\#}$ and ${ }^{\circledR}$ : $\mathrm{p}<0.05 ; * *,{ }^{*}$ and ${ }^{@ @}$ : $\mathrm{P}<0.001$ ). 

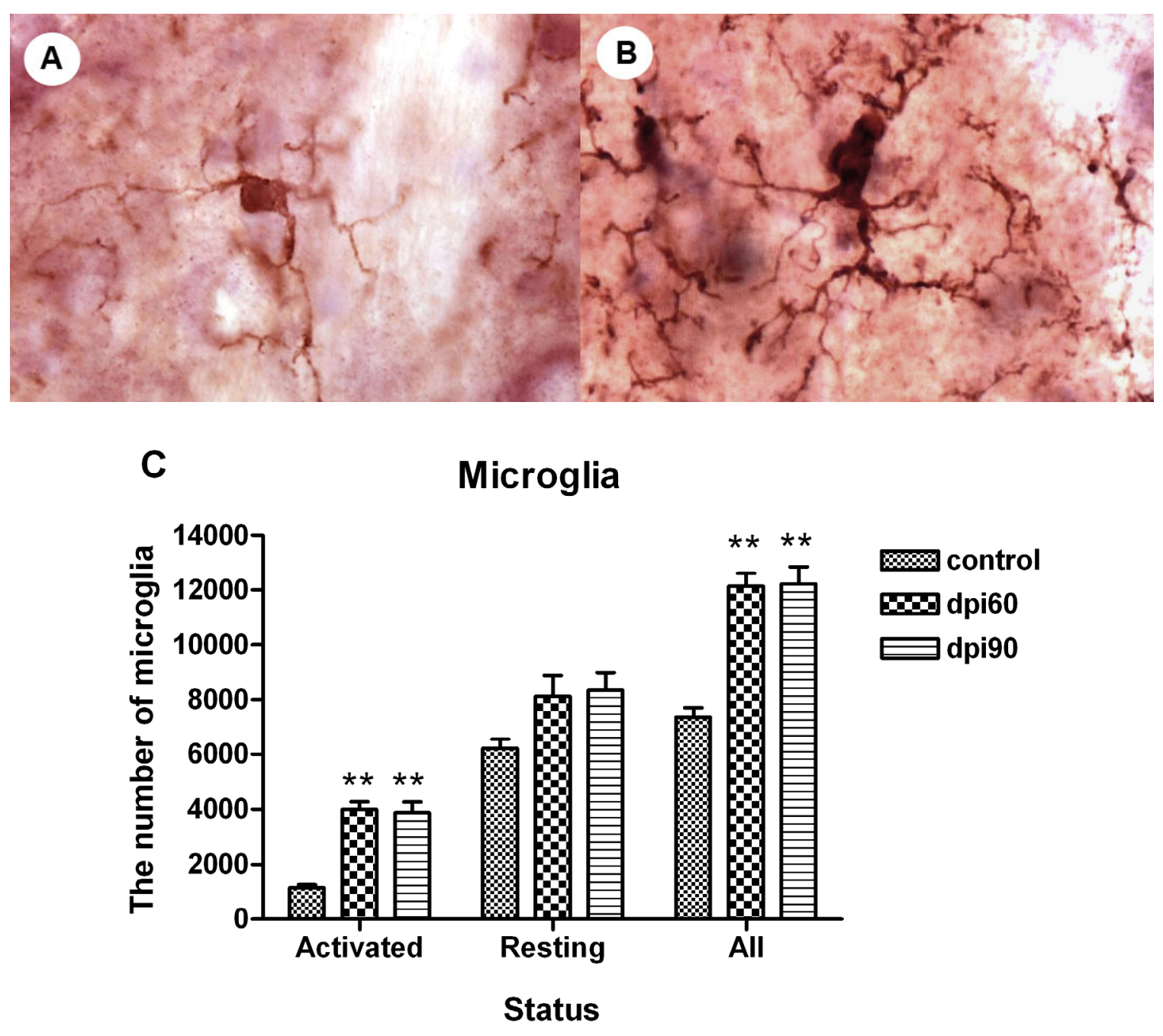

Figure 3.5 H5N1 Infection Increased the Number of Activated Microglia

(A) and (B) represents the typical appearance of resting and activated microglia, respectively. Both resting and activated microglia were observed in the SN of noninfected control and H5N1-infected mice. The number of activated microglia was increased approximately three fold in the H5N1 infected group, compared to that of control (1157 \pm 111 , in the control group; $3996 \pm 283$ (60 dpi) and $3863 \pm 399$ (90 dpi), in H5N1-infected mice, $n=3$ or 4$)$. The total number of microglia was also increased about $67 \%$ in the H5N1-infected group, compared to that of control $(7370 \pm 341$ in the control group; $12140 \pm 467$ (dpi 60) and $12220 \pm 640$ (dpi 90), $\mathrm{n}=3$ or $4, \mathrm{P}<0.001$ ) (C). Statistical significances was determined by one-way ANOVA followed by StudentNewman-Keuls test $(*: \mathrm{p}<0.05, * *: \mathrm{P}<0.001)$. 
3.3.5 Effect of H5N1 infection on levels of cytokines, chemokines and growth factors in the lung and CNS

Activated microglia have been shown to produce a variety of cytokines, chemokines, and growth factors following infection, as well as after other trauma to the CNS. The resulting "inflammatory responses" to these different insults have been shown to be specific to the insult rather than a generalized response to insult (296). Therefore, we examined the cytokine, chemokine and growth factor profiles in regions of the CNS that were infected by H5N1 both during (day 0-10) and after (days 21-90) the acute infectious stage (270). We also measured these proteins in lung, which is the primary site of H5N1 influenza infection in mice (270) in humans (297) to determine if alterations were specific to the CNS or were in response to, or coincident with, peripheral activation of cytokines.

Some cytokines (IL-1 $\alpha$, IL-1 $\beta$, IL-2, IL-9, IL-12, IFN- $\gamma$ and TNF- $\alpha$ ) function primarily to induce inflammation (pro-inflammatory), while other cytokines (IL-6, IL-10, and IL-13) suppress inflammation and are classified as anti-inflammatory (298). Chemokines are cytokines that act as chemoattractants and include eotaxin, KC, IP-10, MCP-1, MIP-1 $\alpha$, and MIP-1 $\beta$ (299). The cytokines acting as growth and differentiation factors that we examined included GM-CSF, M-CSF and VEGF (300). We noted 4 distinct profiles of induction of these factors: First, we observed that some of these proteins transiently increased during the initial phase of infection (through $10 \mathrm{dpi}$ ) then returned to baseline levels. A second pattern of induction showed a transient decrease in expression followed by a return to baseline levels. A third type of cytokine/chemokine expression produced a biphasic pattern, with an initial transient increase in expression, followed by a return to baseline levels and later, a reinduction at times when the influenza virus was no longer detectable by visualization of NP protein. A fourth pattern of cytokine/chemokine expression was observed with no changes in cytokine level during the active phase of infection, but a later induction of cytokines at dpi 60 (Fig 3.6).

In the lung, the expression of cytokines, chemokines and growth factors displayed 3 of the distinct patterns previously described. First, we saw that some of these proteins were transiently increased during the initial phase of infection (through day $10 \mathrm{dpi}$ ) and then returned or decreased to baseline levels. The pro-inflammatory cytokines, chemokines and growth factors that displayed this pattern of expression included IL-6, IL-12, G-CSF, GM-CSF, IFN- $\gamma$, KC, MIP- $1 \alpha$, MIP- $1 \beta$, and TNF- $\alpha$. The antiinflammatory IL-10 also expressed this profile. The second pattern of expression where there is an initial decrease in expression at dpi 10 followed by a return to baseline levels (pattern 2) was displayed by the pro-inflammatory cytokine IL-2. The growth factor, VEGF displayed a third, biphasic pattern of expression with an initial transient increase, followed by a decrease below the baseline level, and another induction at 21, 60 and 90 dpi after the influenza virus was no longer detectable by immunohistochemical labeling of NP protein (pattern 3) (Fig 3.7, Table 3.1). 
Pattern 1

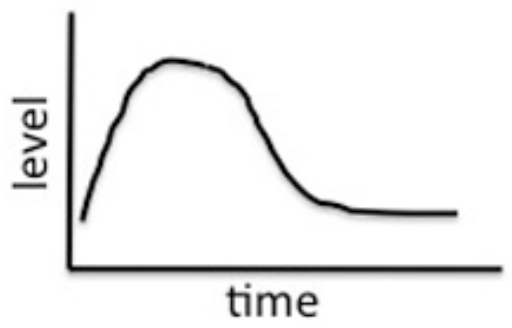

Pattern 2

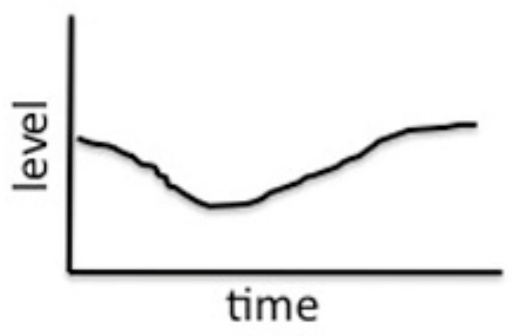

Pattern 3

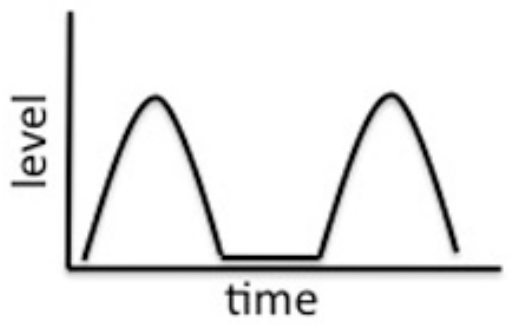

Pattern 4

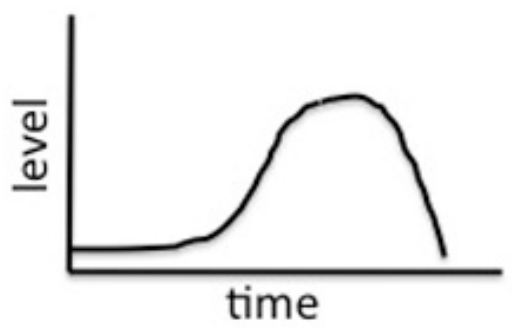

Figure 3.6 Four Distinct Temporal Patterns Seen in Cytokines, Chemokines and Growth Factors Expression Following H5N1 Infection

Four distinct temporal patterns were observed in the expression of cytokines, chemokines and growth factors along the time course after $\mathrm{H} 5 \mathrm{~N} 1$ infection. Pattern 1, a transient increase at initial phase of infection (by day 21) followed by retuning to basal level. Pattern 2, an initial transient decrease in expression followed by a return to baseline levels. Pattern 3, an initial transient increase in expression followed by a return to baseline levels and then a reinduction at 60 days after infection. Pattern 4, no changes during the active phase of infection, but at a later time there was induction. 

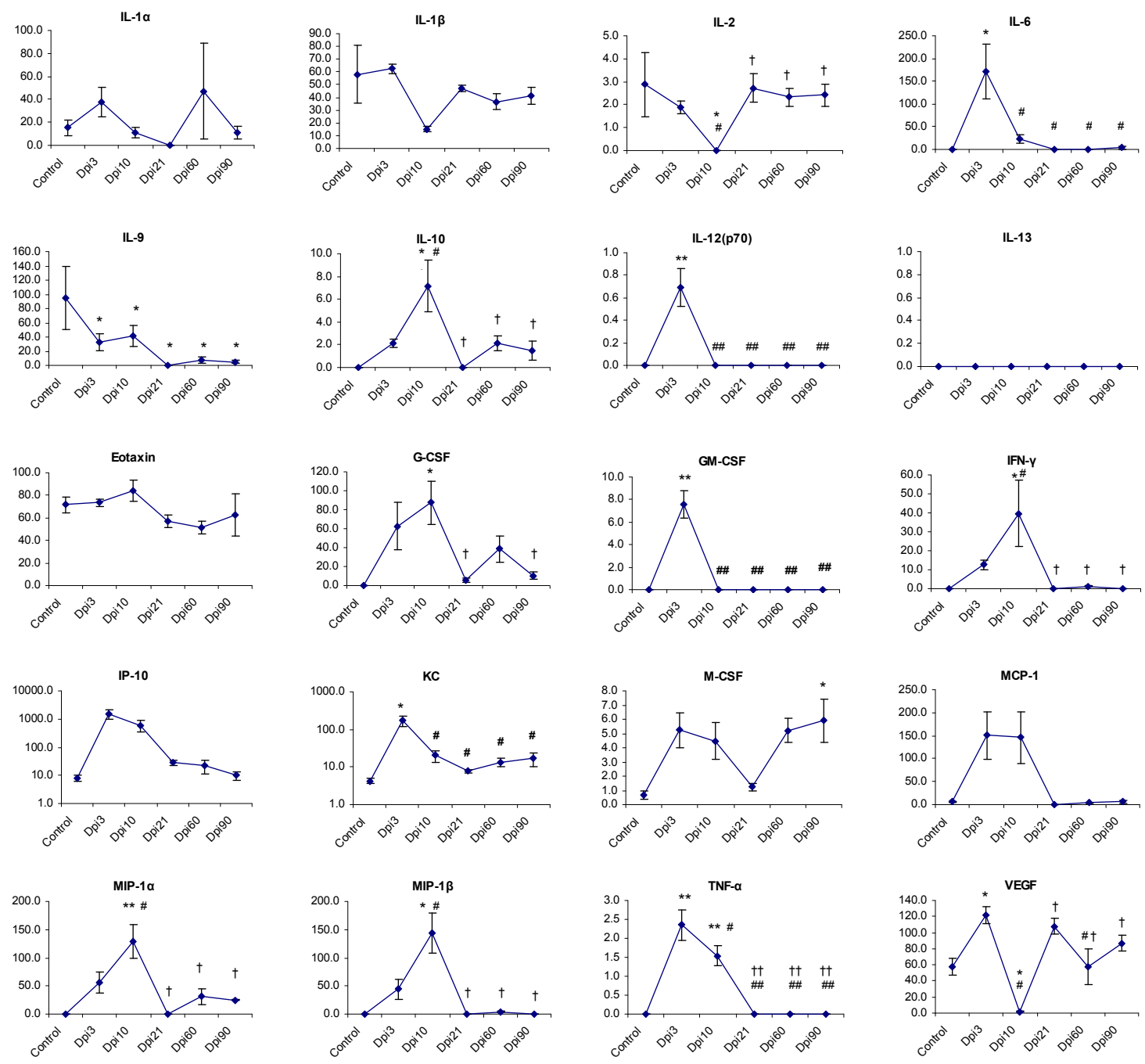

Figure 3.7 Expression of Cytokines, Chemokines and Growth Factors in the Lung Following H5N1 Infection

Two distinct patterns were observed in the lung. The pro-inflammatory cytokines, chemokines and growth factors including IL-6, IL-12, G-CSF, GM-CSF, IFN- $\gamma, \mathrm{KC}$, MIP-1 $\alpha$, MIP-1 $\beta$, and TNF- $\alpha$, and anti-inflammatory IL-10 were increased during the initial phase of infection (through day $10 \mathrm{dpi}$ ) and then returned to baseline levels (pattern 1). The pro-inflammatory cytokine IL-2 showed an initial decrease in expression at 10 dpi followed by a return to baseline levels (pattern 2). The growth factor, VEGF showed an initial transient increase followed by a decrease below the baseline levels and then reinduction at 60 days post infection (pattern 3). Statistical significance was determined by one-way ANOVA followed by Student-Newman-Keuls test. Cytokine levels (pg/25ug of total protein) were presented as mean \pm s.e.m (*: comparison with control mice; ${ }^{*}$ : comparison with 3 dpi mice; ${ }^{\dagger}$ : comparison with 10 dpi mice; ${ }^{@}$ : comparison with 21 dpi

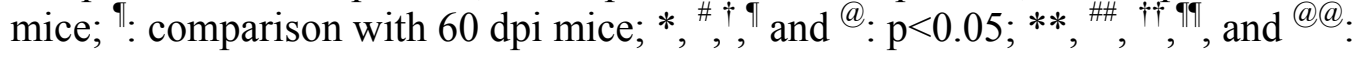
$\mathrm{P}<0.001$; error bars, s.e.m.). 
Table 3.1 Level of Cytokines, Chemokines and Growth Factors in the Lung Following H5N1 Infection

\begin{tabular}{|c|c|c|c|c|c|c|}
\hline Cytokines & Control & Dpi 3 & Dpi 10 & Dpi 21 & Dpi 60 & Dpi 90 \\
\hline IL-1 $\alpha$ & $15.27 \pm 6.66$ & $37.59 \pm 12.72$ & $10.83 \pm 4.80$ & B.D.L & $47.05 \pm 41.94$ & $11.18 \pm 5.63$ \\
\hline IL-1 $\beta$ & $58.05 \pm 22.46$ & $62.36 \pm 3.96$ & $15.23 \pm 2.24$ & $36.04 \pm 12.87$ & $36.66 \pm 6.50$ & $41.26 \pm 6.63$ \\
\hline IL-2 & $2.87 \pm 1.42$ & $1.88 \pm 0.27$ & B.D.L *,\# & $2.80 \pm 0.50^{\dagger}$ & $2.32 \pm 0.40^{\dagger}$ & $2.41 \pm 0.49^{\dagger}$ \\
\hline IL-6 & B.D.L & $171.86 \pm 59.71 *$ & $23.60 \pm 8.89^{\#}$ & B.D.L ${ }^{\#}$ & B.D.L ${ }^{\#}$ & $4.14 \pm 3.84^{\#}$ \\
\hline IL-9 & $95.23 \pm 44.62$ & $32.77 \pm 12.23 *$ & $41.39 \pm 15.36 *$ & B.D.L * & $7.19 \pm 4.06 *$ & $5.14 \pm 2.43 *$ \\
\hline IL-10 & B.D.L & $2.12 \pm 0.36 * \#$ & $7.15 \pm 2.28^{\dagger}$ & B.D.L ${ }^{\dagger}$ & $2.14 \pm 0.63^{\dagger}$ & $1.52 \pm 0.83^{\dagger}$ \\
\hline IL-12(p70) & B.D.L & $0.69 \pm 0.16^{* *}$ & B.D.L ${ }^{\# \#}$ & B.D.L ${ }^{\# \#}$ & B.D.L ${ }^{\# \#}$ & B.D.L ${ }^{\# \#}$ \\
\hline Eotaxin & $71.72 \pm 6.90$ & $73.37 \pm 2.93$ & $83.68 \pm 9.32$ & $43.68 \pm 16.33$ & $51.49 \pm 5.79$ & $62.56 \pm 18.70$ \\
\hline G-CSF & B.D.L & $62.65 \pm 24.75$ & $87.32 \pm 22.77 *$ & $4.96 \pm 2.32$ & $38.57 \pm 13.92$ & $10.51 \pm 3.92$ \\
\hline GM-CSF & B.D.L & $7.58 \pm 1.22 * *$ & B.D.L ${ }^{\# \#}$ & B.D.L ${ }^{\# \#}$ & B.D.L ${ }^{\# \#}$ & B.D.L ${ }^{\# \#}$ \\
\hline IFN- $\gamma$ & B.D.L & $12.69 \pm 2.50$ & $39.64 \pm 17.39 *, \#$ & B.D.L ${ }^{\dagger}$ & $1.33 \pm 0.15^{\dagger}$ & B.D.L ${ }^{\dagger}$ \\
\hline IP-10 & $7.95 \pm 2.00$ & $1507.16 \pm 529.10$ & $602.29 \pm 263.09$ & $22.40 \pm 9.05$ & $22.98 \pm 11.54$ & $9.83 \pm 3.31$ \\
\hline $\mathrm{KC}$ & $4.22 \pm 0.65$ & $171.70 \pm 55.05 *$ & $20.31 \pm 6.87^{\#}$ & $6.59 \pm 1.55^{\#}$ & $13.56 \pm 3.67^{\#}$ & $17.12 \pm 6.75^{\#}$ \\
\hline M-CSF & $0.68 \pm 0.29$ & $5.23 \pm 1.24$ & $4.48 \pm 1.32$ & $1.67 \pm 0.56$ & $5.22 \pm 0.85$ & $5.90 \pm 1.54 *$ \\
\hline MCP-1 & $5.99 \pm 0.65$ & $150.47 \pm 52.41$ & $145.67 \pm 57.29$ & B.D.L & $4.27 \pm 1.41$ & $5.99 \pm 2.94$ \\
\hline
\end{tabular}


Table 3.1 (continued)

\begin{tabular}{lllllll}
\hline Cytokines & Control & Dpi 3 & Dpi 10 & Dpi 21 & Dpi 60 & Dpi 90 \\
\hline MIP-1 $\alpha$ & B.D.L & $56.05 \pm 18.20$ & $128.62 \pm 30.05^{* *, \#}$ & B.D.L ${ }^{\dagger}$ & $31.11 \pm 13.85^{\dagger}$ & $25.07 \pm 1.09^{\dagger}$ \\
MIP-1 $\beta$ & B.D.L & $44.29 \pm 18.00$ & $143.79 \pm 34.82^{* *, \#}$ & B.D.L ${ }^{\dagger \dagger}$ & $4.60 \pm 0.33^{\dagger \dagger}$ & B.D.L ${ }^{\dagger \dagger}$ \\
TNF- $\alpha$ & B.D.L & $2.35 \pm 0.39 * *$ & $1.54 \pm 0.26^{* *, \#}$ & B.D.L ${ }^{\# \#,+\dagger}$ & B.D.L ${ }^{\# \#, \dagger \dagger}$ & B.D.L ${ }^{\# \#, \dagger \dagger}$ \\
VEGF & $57.45 \pm 10.59$ & $121.54 \pm 10.48^{*}$ & $1.72 \pm 0.38^{*, \#}$ & $81.71 \pm 31.21^{\dagger}$ & $57.62 \pm 22.46^{\#, \dagger}$ & $86.88 \pm 9.35^{\dagger}$ \\
\hline
\end{tabular}

Mean fluorescent intensity of blanks, standards, and samples was measured in duplicate wells, using the Luminex 200 reader (Luminex Corp., Austin, TX). The median fluorescent intensity of blanks was subtracted from all readings. Concentration of each cytokine was calculated from a 5-parameter logistic curve obtained from the known concentration of cytokines. All results were normalized with total protein concentration measured from each tissue lysate ( $\mathrm{pg} / 25 \mu \mathrm{g}$ of total protein). B.D.L represented a value below the detectable concentration range of each analyte and was regarded as zero for statistical calculation. Statistical significance was determined by one-way ANOVA followed by Student-Newman-Keuls test (*: comparison with control mice; ${ }^{\#}$ : comparison with

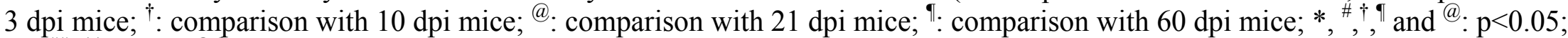
**, ${ }^{\#}, \dagger$, ${ }^{\dagger \uparrow}$, and ${ }^{@ @: ~} \mathrm{P}<0.001$; mean number \pm s.e.m.). 
In the CNS, we examined the expression of cytokines, chemokines and growth factors in 4 separate regions: brainstem, substantia nigra, striatum and cortex. In the brainstem, the expression of cytokines, chemokines and growth factors displayed 2 of the different patterns described above. The pro-inflammatory cytokines, chemokines and growth factors that expressed the first profile were IL-1 $\alpha$, IL-12 (p70), IL-13, eotaxin, GCSF, GM-CSF, IP-10, KC, M-CSF, MCP-1, MIP-1 $\alpha$, MIP-1 $\beta$, and TNF- $\alpha$. The antiinflammatory cytokines IL-10 also followed this profile. The level of proinflammatory cytokines and growth factors IL-1 $\beta$, IL-2, and VEGF displayed pattern 4 . They were not changed immediately upon detection of the virus, but increased later when NP protein was no longer evident in the region. (Fig 3.8, Table 3.2).

In the substantia nigra, the expression of cytokines, chemokines and growth factors displayed profiles 1,3 , and 4, described above. The pro-inflammatory cytokines, chemokines and growth factors that expressed the first profile were IL-1 $\beta$, IL-2, IL-6, GCSF, M-CSF, and MCP-1 where their expression increased at 3 or $10 \mathrm{dpi}$ and then returned to baseline levels. IL-13 exhibited the third pattern listed above, with its levels increasing prior to day 21 followed by a return to baseline and another rise in level at a later point after the active infection (60 dpi) was over. SN levels of the chemokines and growth factors GM-CSF and MIP-1 $\beta$ exhibited pattern 4 , where expression did not change immediately upon detection of the virus, but increased later when NP protein was no longer evident in the region. Neither MIP-1 $\alpha$ nor TNF- $\alpha$ was detected in the SN following exposure to influenza (Fig 3.9, Table 3.3).

In striatum, the expression of cytokines, chemokines and growth factors displayed profiles 1, 3, and 4, described above. The pro-inflammatory chemokines and growth factors that expressed profile 1 were eotaxin and M-CSF. The cytokine that expressed profile 3 was IL-2, while the anti-inflammatory IL-10 displayed profile 4. IL-1 $\alpha$, IL-6, IL-12 (p70), IL-13, G-CSF, GM-CSF, IFN- $\gamma$, MIP-1 $\alpha$, MIP-1 $\beta$, and TNF- $\alpha$ were not detected in striatum following exposure to H5N1 influenza (Fig 3.10, Table 3.4).

In cortex, the expression of cytokines, chemokines and growth factors displayed profiles 1, 2 and 4 . The pro-inflammatory cytokines that expressed profile 1 were IL-2 and IL-9. The growth factor VEGF expressed profile 2 while an anti-inflammatory cytokine, IL-10 displayed profile 4. IL-1 $\alpha$, IL-6, IL-12 (p70), IL-13, G-CSF, GM-CSF, IFN- $\gamma$, MIP-1 $\alpha$, MIP-1 $\beta$, or TNF- $\alpha$ were not detected in cortex following exposure to influenza (Fig 3.11, Table 3.5).

\subsection{Discussion}

Our results demonstrate that the A/VN/1203/04 strain of the H5N1 influenza virus can induce both short-term and long-term effects in the central nervous system. During the acute phase of the infection, which lasts through day 10 post-infection (270), the virus induces a transient reduction of dopamine production in the basal ganglia. Co-incident to this loss of dopamine we observe an immediate and long-lasting increase in inflammation 

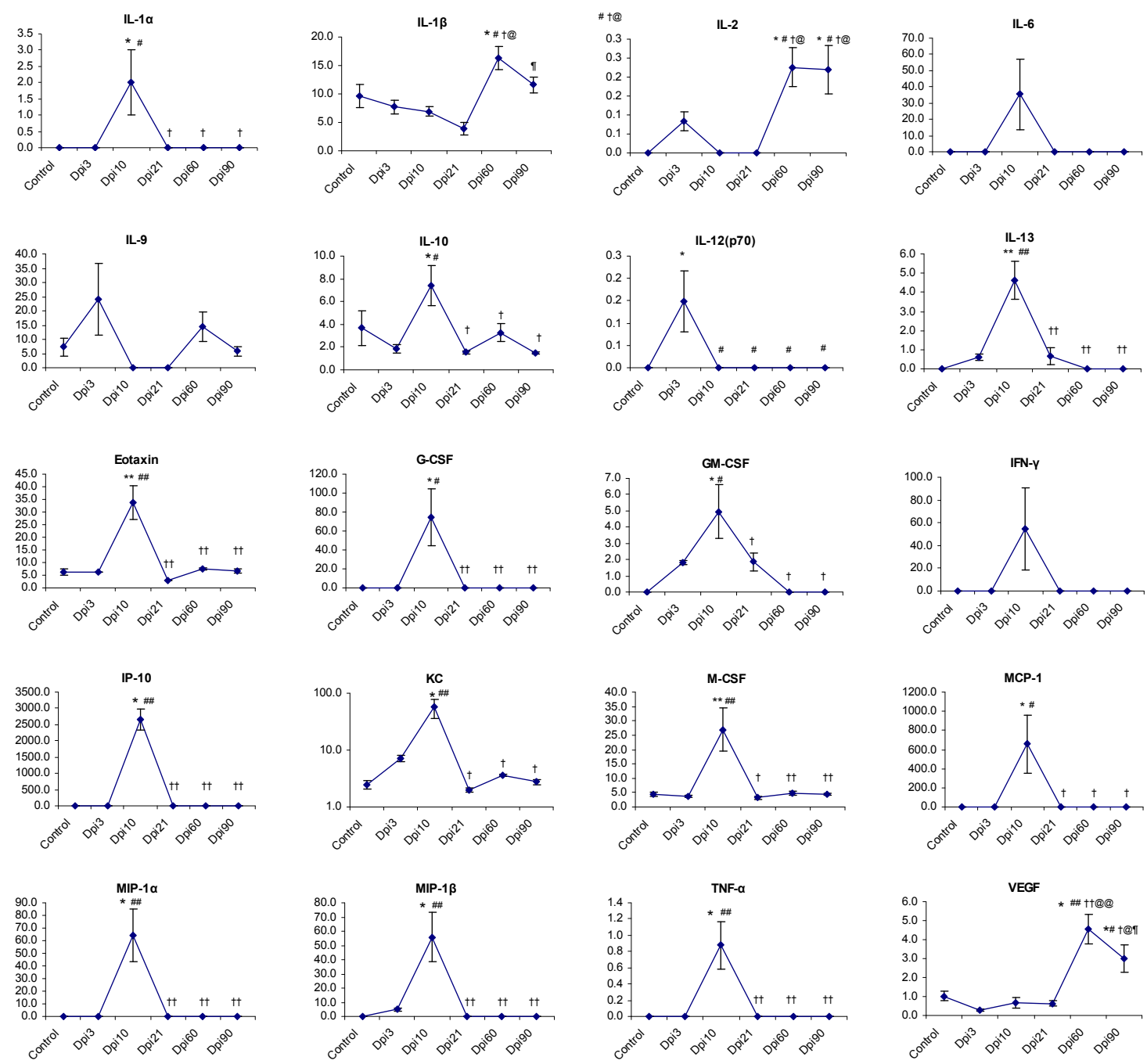

Figure 3.8 Expression of Cytokines, Chemokines and Growth Factors in the Brainstem Following H5N1 Infection

Three distinct patterns were observed in the brainstem. The pro-inflammatory cytokines, chemokines and growth factors including IL-1 $\alpha$, IL-12(p70), IL-13, eotaxin, G-CSF, GM-CSF, IP-10, KC, M-CSF, MCP-1, MIP- $1 \alpha$, MIP-1 $\beta$, and TNF- $\alpha$ and antiinflammatory IL-10 were increased during the initial phase of infection (through day 10 dpi) and then returned or decreased to baseline levels (pattern 1). The pro-inflammatory cytokines, chemokines and growth factors including IL-1 $\beta$, IL-2 and VEGF did not show changes during the active phase of infection, but at a later time, displayed induction (pattern 4). Statistical significance was determined by one-way ANOVA followed by Student-Newman-Keuls test and cytokine levels ( $\mathrm{pg} / 25 \mathrm{ug}$ of total protein) were presented as mean \pm s.e.m (*: comparison with control mice; ${ }^{\#}$ : comparison with 3 dpi mice; ${ }^{\dagger}$ : comparison with 10 dpi mice; ${ }^{\circledR}$ : comparison with 21 dpi mice; ${ }^{\natural}$ : comparison with 60 dpi

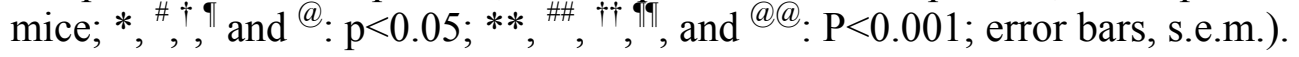


Table 3.2 Level of Cytokines, Chemokines and Growth Factors in the Brainstem Following H5N1 Infection

\begin{tabular}{|c|c|c|c|c|c|c|}
\hline Cytokines & Control & Dpi 3 & Dpi 10 & Dpi 21 & Dpi 60 & Dpi 90 \\
\hline IL- $1 \beta$ & $9.71 \pm 2.03$ & $7.70 \pm 1.22$ & $6.91 \pm 0.84$ & $3.89 \pm 1.03$ & $16.29 \pm 1.99 *, \#, \uparrow, @$ & $11.62 \pm 1.35^{\natural}$ \\
\hline IL-2 & B.D.L & $0.08 \pm 0.02$ & B.D.L & B.D.L & $0.23 \pm 0.05 *, \#, \dagger, @$ & $0.22 \pm 0.06 *, \#, \dot{\dagger} @$ \\
\hline IL-6 & B.D.L & B.D.L & $35.53 \pm 21.63$ & B.D.L & B.D.L & B.D.L \\
\hline IL-10 & $3.68 \pm 1.55$ & $1.85 \pm 0.39$ & $7.44 \pm 1.76^{*, \#}$ & $1.56 \pm 0.14^{\dagger}$ & $3.27 \pm 0.81^{\dagger}$ & $1.49 \pm 0.11^{\dagger}$ \\
\hline IL-12(p70) & B.D.L & $0.15 \pm 0.70 *$ & B.D.L ${ }^{\#}$ & B.D.L ${ }^{\#}$ & B.D.L ${ }^{\#}$ & B.D.L ${ }^{\#}$ \\
\hline IL-13 & B.D.L & $0.60 \pm 0.15$ & $4.61 \pm 0.99 * *, \# \#$ & $0.67 \pm 0.44^{\dagger \dagger}$ & B.D.L ${ }^{\dagger \dagger}$ & B.D.L ${ }^{\dagger \dagger}$ \\
\hline GM-CSF & B.D.L & $1.82 \pm 0.13$ & $4.94 \pm 1.65 *$ & $1.85 \pm 0.54$ & B.D.L & B.D.L \\
\hline $\mathrm{KC}$ & $2.47 \pm 0.44$ & $7.06 \pm 0.87$ & $56.88 \pm 20.29 * \# \#$ & $2.00 \pm 0.16^{\dagger}$ & $3.61 \pm 0.14^{\dagger}$ & $2.73 \pm 0.30^{\dagger}$ \\
\hline IFN- $\gamma$ & B.D.L & B.D.L & $54.92 \pm 35.98$ & B.D.L & B.D.L & B.D.L \\
\hline IP-10 & B.D.L & $3.57 \pm 2.45$ & $2661.73 \pm 322.66 * *$ & $8.55 \pm 2.65$ & $9.64 \pm 4.13$ & B.D.L \\
\hline M-CSF & $4.28 \pm 0.76$ & $3.55 \pm 0.42$ & $26.94 \pm 7.61 * *$ \#\# & $3.13 \pm 0.68^{\dagger}$ & $4.79 \pm 0.59^{\dagger}$ & $4.42 \pm 0.38^{\dagger \dagger}$ \\
\hline
\end{tabular}


Table 3.2 (continued)

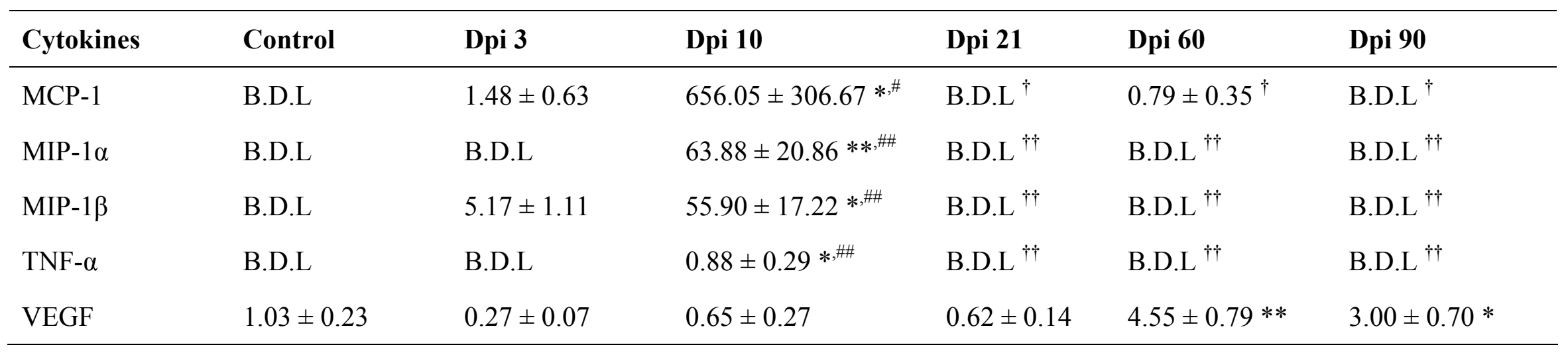

Mean fluorescent intensity of blanks, standards, and samples was measured in duplicate wells, using the Luminex 200 reader (Luminex Corp., Austin, TX). The median fluorescent intensity of blanks was subtracted from all readings. Concentration of each cytokine was calculated from a 5-parameter logistic curve obtained from the known concentration of cytokines. All results were normalized with total protein concentration measured from each tissue lysate ( $\mathrm{pg} / 25 \mu \mathrm{g}$ of total protein). B.D.L represented a value below the detectable concentration range of each analyte and was regarded as zero for statistical calculation. Statistical significance was determined by one-way ANOVA followed by Student-Newman-Keuls test (*: comparison with control mice; ${ }^{*}$ : comparison with 3 dpi mice; ${ }^{\dagger}$ : comparison with 10 dpi mice; ${ }^{\circledR}$ : comparison with 21 dpi mice; ${ }^{\uparrow}$ : comparison with 60 dpi mice; ${ }^{*},{ }^{\#},{ }^{\dagger},{ }^{\natural}$ and ${ }^{@}:$ p $<0.05$; $* *,{ }^{*},{ }^{\dagger},{ }^{\uparrow \uparrow}$, and ${ }^{@ @: ~} \mathrm{P}<0.001$; mean number \pm s.e.m. $)$. 

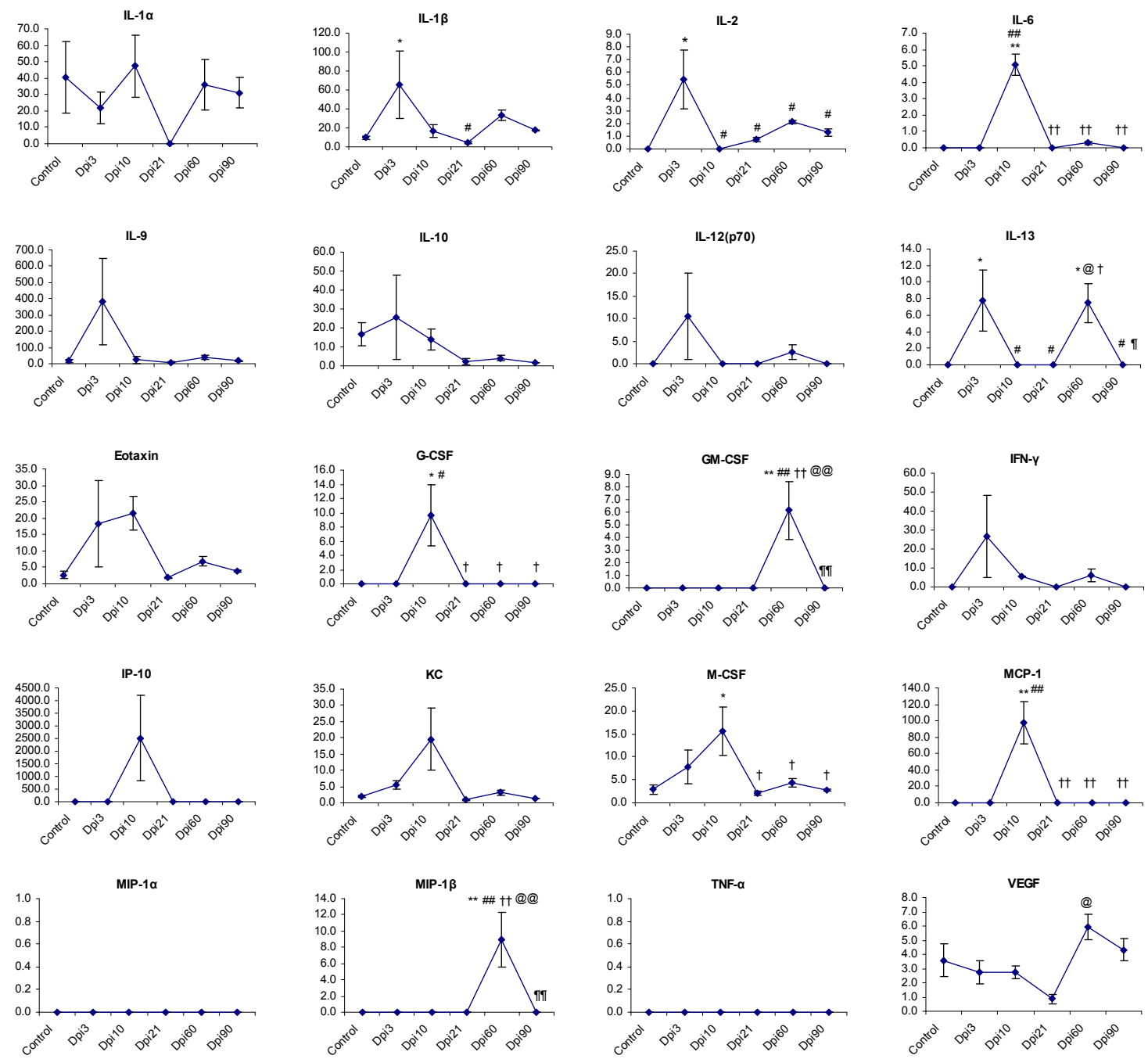

Figure 3.9 Expression Cytokines, Chemokines and Growth Factors in the SN Following H5N1 Infection

In substantia nigra, the pro-inflammatory cytokines/chemokines, including IL- $1 \beta$, IL-2, IL-6, G-CSF, M-CSF, and MCP-1 were increased during the initial phase of infection (through day $10 \mathrm{dpi}$ ) and then returned or decreased to baseline levels (pattern 1). The pro-inflammatory cytokine IL-13 was initially increased and then returned to baseline levels prior to reinduction at 60 dpi (pattern 3). The pro-inflammatory chemokine MIP-1 $\beta$ and growth factor GM-CSF did not show changes during the active phase of infection (through day $10 \mathrm{dpi}$ ), but at a later time, displayed induction (pattern 4). Statistical significance was determined by one-way ANOVA followed by Student-Newman-Keuls test and cytokine levels (pg/25ug of total protein) were presented as mean \pm s.e.m (*: comparison with control mice; ${ }^{\#}$ : comparison with 3 dpi mice; ${ }^{\dagger}$ : comparison with 10 dpi mice; ${ }^{\circledR}$ : comparison with 21 dpi mice; ${ }^{\top}$ : comparison with $60 \mathrm{dpi}$ mice; * ${ }^{*},+,{ }^{\natural}$ and ${ }^{\circledR}$ : $\mathrm{p}<0.05 ; * *,{ }^{\#},{ }^{\dagger},{ }^{\uparrow \uparrow}$, and ${ }^{@ @}: \mathrm{P}<0.001$; error bars, s.e.m.). 
Table 3.3 Level of Cytokines, Chemokines and Growth Factors in the SN Following H5N1 Infection

\begin{tabular}{|c|c|c|c|c|c|c|}
\hline Cytokines & Control & Dpi 3 & Dpi 10 & Dpi 21 & Dpi 60 & Dpi 90 \\
\hline $\mathrm{IL}-1 \alpha$ & $40.27 \pm 21.73$ & $22.05 \pm 9.73$ & $47.36 \pm 18.78$ & B.D.L & $35.87 \pm 15.55$ & $30.99 \pm 9.27$ \\
\hline $\mathrm{IL}-1 \beta$ & $9.65 \pm 1.90$ & $65.72 \pm 35.21 *$ & $16.57 \pm 6.40$ & $4.61 \pm 1.39^{\#}$ & $33.34 \pm 5.08$ & $17.27 \pm 0.78$ \\
\hline IL-2 & B.D.L & $5.41 \pm 2.31 *$ & B.D.L ${ }^{\#}$ & $0.74 \pm 0.13^{\#}$ & $2.13 \pm 0.14^{\#}$ & $1.28 \pm 0.29^{\#}$ \\
\hline IL-6 & B.D.L & B.D.L & $5.08 \pm 0.64 * *, \# \#$ & B.D.L ${ }^{\dagger \dagger}$ & $0.29 \pm 0.11^{\dagger}$ & B.D.L ${ }^{\dagger \dagger}$ \\
\hline IL-9 & $16.39 \pm 7.15$ & $381.82 \pm 264.60$ & $23.66 \pm 20.63$ & $9.04 \pm 2.12$ & $41.86 \pm 13.06$ & $16.79 \pm 3.71$ \\
\hline IL-10 & $16.90 \pm 6.08$ & $25.52 \pm 22.40$ & $13.88 \pm 5.35$ & $2.41 \pm 1.57$ & $3.90 \pm 1.37$ & $1.66 \pm 0.06$ \\
\hline IL-12(p70) & B.D.L & $10.53 \pm 9.65$ & B.D.L & B.D.L & $2.56 \pm 1.57$ & B.D.L \\
\hline IL-13 & B.D.L & $7.78 \pm 3.68 *$ & B.D.L ${ }^{\#}$ & B.D.L ${ }^{\#}$ & $7.47 \pm 2.38 *, @, \dagger$ & B.D.L ${ }^{\#, \Phi}$ \\
\hline Eotaxin & $2.69 \pm 1.08$ & $18.31 \pm 13.08$ & $21.46 \pm 5.06$ & $1.91 \pm 0.17$ & $6.84 \pm 1.43$ & $3.86 \pm 0.34$ \\
\hline G-CSF & B.D.L & B.D.L & $9.58 \pm 4.28 *$ *\# & B.D.L ${ }^{\dagger}$ & B.D.L ${ }^{\dagger}$ & B.D.L ${ }^{\dagger}$ \\
\hline GM-CSF & B.D.L & B.D.L & B.D.L & B.D.L & $6.15 \pm 2.29 * *, \# \#, \dagger \dagger, @ @$ & B.D.L \\
\hline IFN- $\gamma$ & B.D.L & $26.54 \pm 21.72$ & $5.59 \pm 0.17$ & B.D.L & $6.06 \pm 3.37$ & B.D.L \\
\hline IP-10 & $3.63 \pm 1.41$ & $8.70 \pm 2.72$ & $2519.40 \pm 1700.01$ & $3.06 \pm 1.14$ & $12.08 \pm 0.68$ & B.D.L \\
\hline $\mathrm{KC}$ & $1.95 \pm 0.42$ & $5.55 \pm 1.34$ & $19.57 \pm 9.65$ & $0.83 \pm 0.15$ & $3.12 \pm 0.69$ & $1.27 \pm 0.05$ \\
\hline MCP-1 & B.D.L & B.D.L & $97.72 \pm 25.34 * *, \# \#$ & B.D.L ${ }^{\dagger \dagger}$ & B.D.L ${ }^{\dagger \dagger}$ & B.D.L ${ }^{\dagger \dagger}$ \\
\hline MIP-1 $\beta$ & B.D.L & B.D.L & B.D.L & B.D.L & 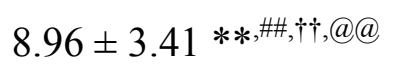 & B.D.L $\uparrow$ \\
\hline
\end{tabular}


Table 3.3 (continued)

\begin{tabular}{lllllll}
\hline Cytokines & Control & Dpi 3 & Dpi 10 & Dpi 21 & Dpi 60 & Dpi 90 \\
\hline M-CSF & $2.90 \pm 1.02$ & $7.84 \pm 3.70$ & $15.53 \pm 5.29^{*}$ & $2.16 \pm 0.46^{\dagger}$ & $4.40 \pm 0.96^{\dagger}$ & $2.75 \pm 0.23^{\dagger}$ \\
VEGF & $3.59 \pm 1.13$ & $2.73 \pm 0.84$ & $2.74 \pm 0.44$ & $0.86 \pm 0.32$ & $5.94 \pm 0.88^{@}$ & $4.33 \pm 0.78$ \\
\hline
\end{tabular}

Mean fluorescent intensity of blanks, standards, and samples was measured in duplicate wells, using the Luminex 200 reader (Luminex Corp., Austin, TX). The median fluorescent intensity of blanks was subtracted from all readings and concentration of each cytokine was calculated from a 5-parameter logistic curve obtained from the known concentration of cytokines. All results were normalized with total protein concentration measured from each tissue lysate ( $\mathrm{pg} / 25 \mu \mathrm{g}$ of total protein). B.D.L represented a value below the detectable concentration range of each analyte and was regarded as zero for statistical calculation. Statistical significance was determined by one-way ANOVA followed by Student-Newman-Keuls test $\left(*\right.$ : comparison with control mice; ${ }^{*}$ : comparison with 3 dpi mice; ${ }^{\dagger}$ : comparison with 10 dpi mice; ${ }^{\circledR}$ : comparison with 21 dpi mice; ${ }^{\uparrow}:$ comparison with 60 dpi mice; *, $,{ }^{\sharp},{ }^{\uparrow}$ and ${ }^{\circledR}$ : p $<0.05$; **, $,{ }^{\#}, \dagger,{ }^{\dagger \uparrow}$, and ${ }^{@ @}: \mathrm{P}<0.001$; mean number \pm s.e.m.). 

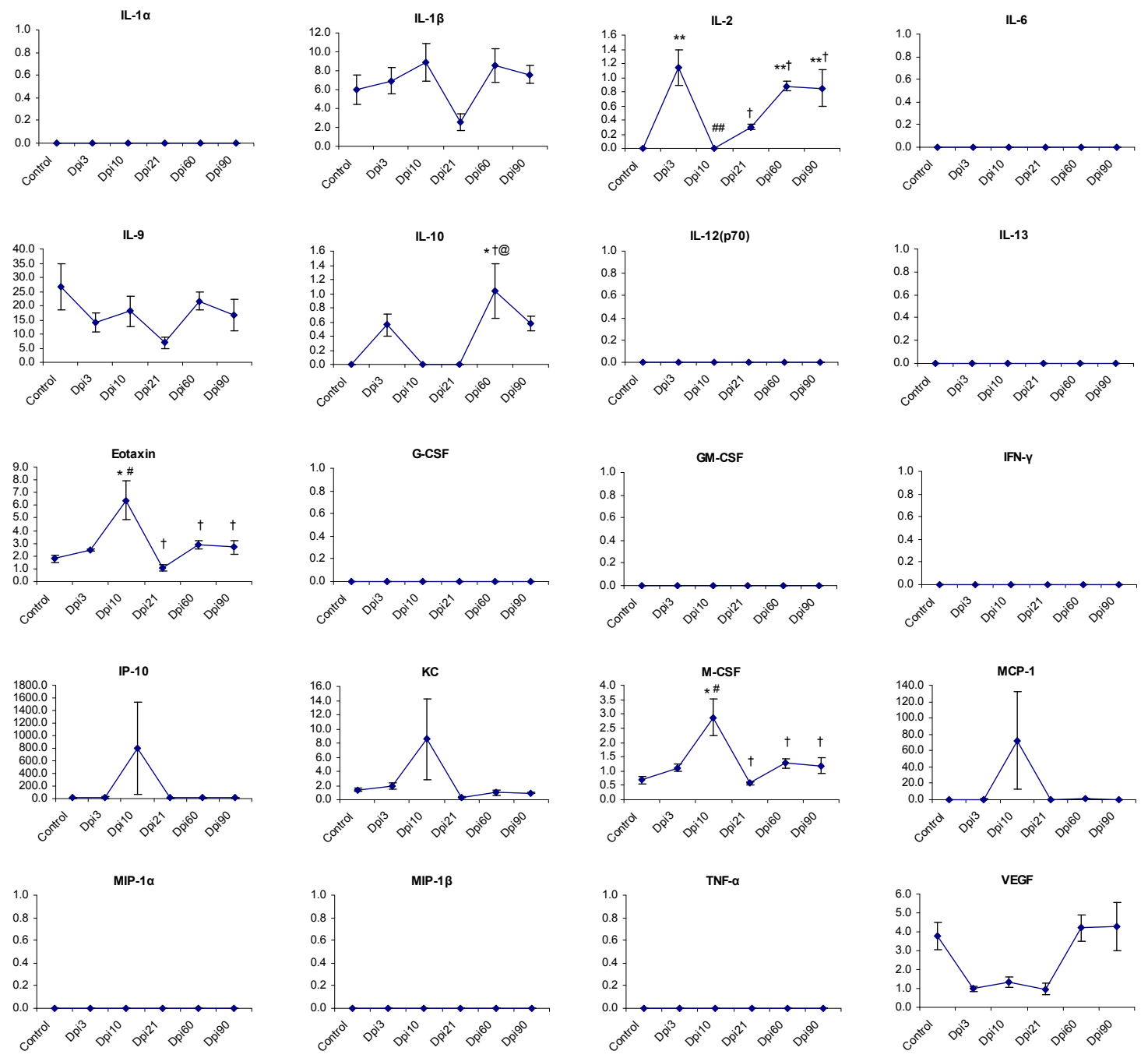

Figure 3.10 Expression of Cytokines, Chemokines and Growth Factors in the Striatum Following H5N1 Infection

In striatum, the pro-inflammatory chemokines and growth factors including eotaxin and M-CSF were increased during the initial phase of infection (through day $10 \mathrm{dpi}$ ) and then returned to baseline levels (pattern 1). The pro-inflammatory cytokine IL-2 was initially increased and then returned to baseline levels after which it reinduced at 60 dpi (pattern 3 ). The anti-inflammatory cytokine IL-10 did not show changes during the active phase of infection, but at a later time, displayed induction (pattern 4). Statistical significance was determined by one-way ANOVA followed by Student-Newman-Keuls test and cytokine levels (pg/25ug of total protein) were presented as mean \pm s.e.m (*: comparison with control mice; ${ }^{\#}$ : comparison with 3 dpi mice; ${ }^{\dagger}$ : comparison with 10 dpi mice; ${ }^{\circledR}$ : comparison with 21 dpi mice; ๆ: comparison with 60 dpi mice; $*{ }^{*}{ }^{\dagger},{ }^{\uparrow}$ and ${ }^{\circledR}: p<0.05 ; * *$, \#\#, ${ }^{\dagger \uparrow}$, , and ${ }^{@ @: ~} \mathrm{P}<0.001$; error bars, s.e.m.). 
Table 3.4 Level of Cytokines, Chemokines and Growth Factors in the Striatum Following H5N1 Infection

\begin{tabular}{|c|c|c|c|c|c|c|}
\hline Cytokines & Control & Dpi 3 & Dpi 10 & Dpi 21 & Dpi 60 & Dpi 90 \\
\hline $\mathrm{IL}-1 \beta$ & $6.05 \pm 1.55$ & $6.94 \pm 1.41$ & $8.91 \pm 2.03$ & $2.67 \pm 0.75$ & $8.58 \pm 1.77$ & $7.61 \pm 0.97$ \\
\hline IL-2 & B.D.L & $1.14 \pm 0.25 * *$ & B.D.L ${ }^{\# \#}$ & $0.97 \pm 0.78^{\dagger}$ & $0.88 \pm 0.07 * *, \dagger$ & $0.84 \pm 0.25 * *$, \\
\hline IL-9 & $26.63 \pm 8.05$ & $13.99 \pm 3.26$ & $18.12 \pm 5.37$ & $5.94 \pm 2.04$ & $21.66 \pm 3.18$ & $16.58 \pm 5.51$ \\
\hline IL-10 & B.D.L & $0.56 \pm 0.16$ & B.D.L & B.D.L & $1.04 \pm 0.38 *, \dagger, @$ & $0.58 \pm 0.11$ \\
\hline Eotaxin & $1.78 \pm 0.27$ & $2.46 \pm 0.09$ & $6.38 \pm 1.50 * \#$ & $1.57 \pm 0.58^{\dagger}$ & $2.89 \pm 0.35^{\dagger}$ & $2.69 \pm 0.52^{\dagger}$ \\
\hline IP-10 & $11.17 \pm 8.18$ & $19.02 \pm 14.65$ & $794.54 \pm 734.04$ & $8.03 \pm 7.42$ & $12.53 \pm 10.74$ & $12.48 \pm 6.23$ \\
\hline $\mathrm{KC}$ & $1.36 \pm 0.21$ & $1.98 \pm 0.45$ & $8.53 \pm 5.74$ & $1.02 \pm 0.76$ & $0.98 \pm 0.39$ & $0.95 \pm 0.07$ \\
\hline MCP-1 & B.D.L & $0.61 \pm 0.14$ & $72.37 \pm 59.88$ & B.D.L & $1.24 \pm 0.36$ & B.D.L \\
\hline $\mathrm{M}-\mathrm{CSF}$ & $0.68 \pm 0.14$ & $1.12 \pm 0.12$ & $2.88 \pm 0.64 *$ *\# & $1.19 \pm 0.70^{\dagger}$ & $1.27 \pm 0.16^{\dagger}$ & $1.18 \pm 0.27^{\dagger}$ \\
\hline VEGF & $3.76 \pm 0.72$ & $0.97 \pm 0.11$ & $1.34 \pm 0.30$ & $1.48 \pm 0.63$ & $4.20 \pm 0.69$ & $4.29 \pm 1.29$ \\
\hline
\end{tabular}

Mean fluorescent intensity of blanks, standards, and samples was measured in duplicate wells, using the Luminex 200 reader (Luminex Corp., Austin, TX). The median fluorescent intensity of blanks was subtracted from all readings. Concentration of each cytokine was calculated from a 5-parameter logistic curve obtained from the known concentration of cytokines. All results were normalized with total protein concentration measured from each tissue lysate (pg/25 $\mu \mathrm{g}$ of total protein). B.D.L represented a value below the detectable concentration range of each analyte and was regarded as zero for statistical calculation. Statistical significance was determined by one-way ANOVA followed by Student-Newman-Keuls test (*: comparison with control mice; ${ }^{*}$ : comparison with 3 dpi mice; ${ }^{\dagger}$ : comparison with 10 dpi mice; ${ }^{\circledR}$ : comparison with 21 dpi mice; ${ }^{\uparrow}$ : comparison with 60 dpi mice; *, $,{ }^{\dagger},{ }^{\uparrow}$ and ${ }^{@}:$ p $<0.05$; **, $,{ }^{\dagger \dagger},{ }^{\uparrow \uparrow}$, and ${ }^{@ @: ~} \mathrm{P}<0.001$; mean number \pm s.e.m.). 

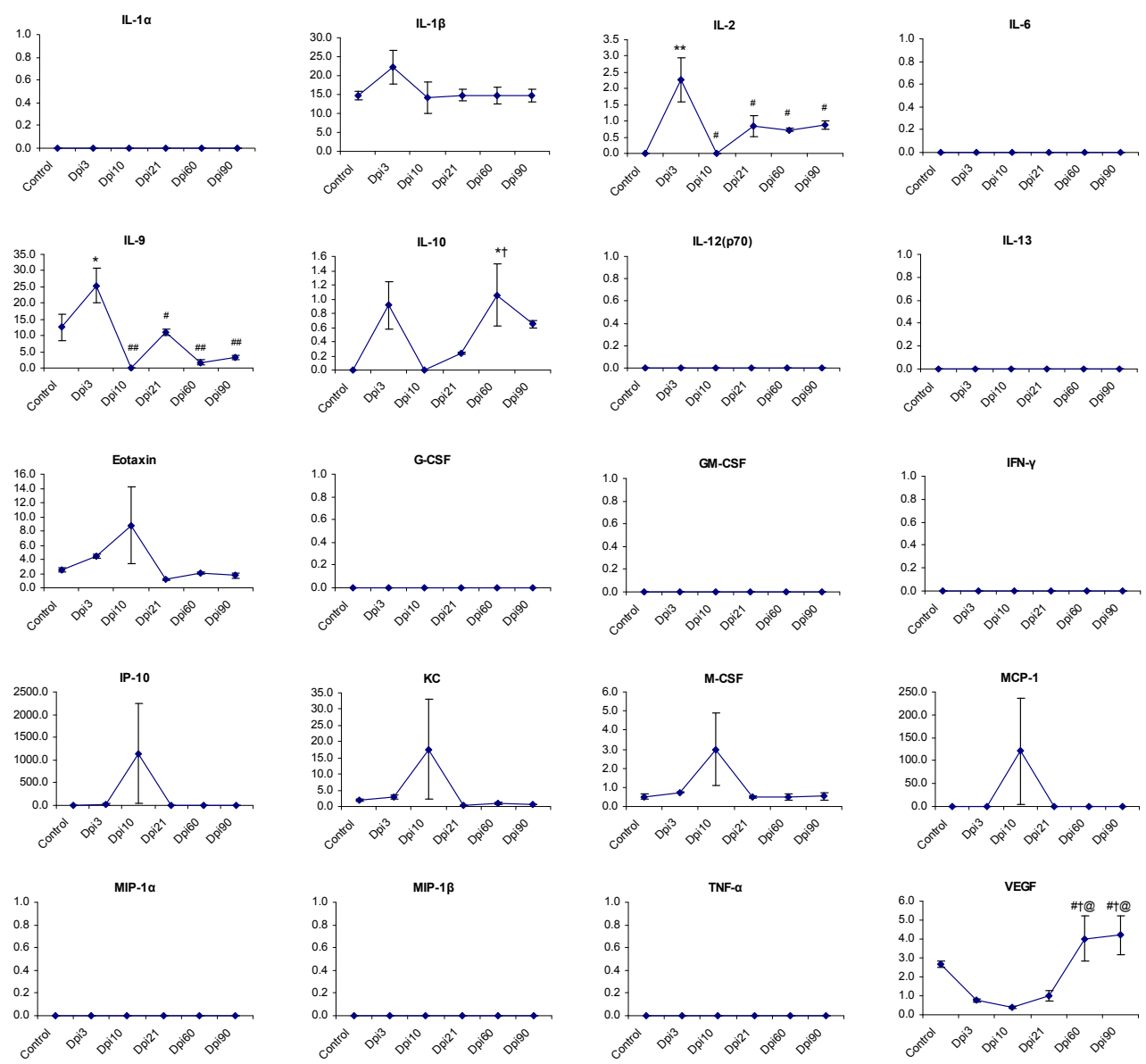

Figure 3.11 Expression of Cytokines, Chemokines and Growth Factors in the Cortex Following H5N1 Infection

In cortex, the pro-inflammatory cytokines, chemokines and growth factors including IL$\beta$, IL-9, eotaxin, IP-10, KC, M-CSF and MCP-1 were increased during the initial phase of infection (through day $10 \mathrm{dpi}$ ) and then returned or decreased to baseline levels (pattern 1). The pro-inflammatory cytokine IL-2 and anti-inflammatory IL-10 were initially increased and returned to baseline levels and then reinduced at 60 days post infection (pattern 3). The pro-inflammatory growth factor VEGF didn't show changes during the active phase of infection, but at a later time, displayed induction (pattern 4). IL-1 $\alpha$, IL-6, IL-12, IL-13, G-CSF, GM-CSF, IFN- $\gamma$, MIP-1 $\alpha$, MIP-1 $\beta$, or TNF- $\alpha$ were not detected in cortex following exposure to influenza. Statistical significance was determined by oneway ANOVA followed by Student-Newman-Keuls test and cytokine levels (pg/25ug of total protein) were presented as mean \pm s.e.m (*: comparison with control mice; ${ }^{\#}$ : comparison with 3 dpi mice; ${ }^{\dagger}$ : comparison with 10 dpi mice; ${ }^{@}$ : comparison with $21 \mathrm{dpi}$

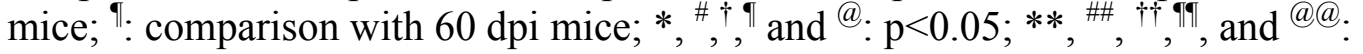
$\mathrm{P}<0.001$; error bars, s.e.m.). 
Table 3.5 Level of Cytokines, Chemokines and Growth Factors in the Cortex Following H5N1 Infection

\begin{tabular}{|c|c|c|c|c|c|c|}
\hline Cytokines & Control & Dpi 3 & Dpi 10 & Dpi 21 & Dpi 60 & Dpi 90 \\
\hline IL-1 $\beta$ & $14.71 \pm 1.12$ & $22.26 \pm 4.52$ & $14.18 \pm 4.14$ & $11.83 \pm 3.62$ & $14.73 \pm 2.26$ & $14.82 \pm 1.68$ \\
\hline IL-2 & B.D.L & $2.28 \pm 0.68 * *$ & B.D.L ${ }^{\#}$ & $1.39 \pm 0.67^{\#}$ & $0.73 \pm 0.06^{\#}$ & $0.88 \pm 0.12^{\#}$ \\
\hline IL-9 & $12.54 \pm 3.97$ & $25.42 \pm 5.45 *$ & B.D.L \#\# & $9.02 \pm 2.43^{\#}$ & $1.76 \pm 0.81$ & $3.19 \pm 0.73^{\# \#}$ \\
\hline IL-10 & B.D.L & $0.92 \pm 0.33$ & B.D.L & $0.82 \pm 0.72$ & $1.06 \pm 0.43 *, \dagger$ & $0.65 \pm 0.05$ \\
\hline Eotaxin & $2.55 \pm 0.27$ & $4.46 \pm 0.35$ & $8.76 \pm 5.39$ & $1.60 \pm 0.54$ & $2.08 \pm 0.20$ & $1.71 \pm 0.35$ \\
\hline IP-10 & $1.11 \pm 0.30$ & $19.18 \pm 17.64$ & $1140.80 \pm 1103.95$ & $1.46 \pm 0.41$ & B.D.L & B.D.L \\
\hline $\mathrm{KC}$ & $1.95 \pm 0.27$ & $2.84 \pm 0.70$ & $17.60 \pm 15.38$ & $0.98 \pm 0.78$ & $0.97 \pm 0.23$ & $0.64 \pm 0.09$ \\
\hline MCP-1 & B.D.L & $0.52 \pm 0.05$ & $120.64 \pm 116.40$ & B.D.L & B.D.L & B.D.L \\
\hline M-CSF & $0.52 \pm 0.12$ & $0.73 \pm 0.03$ & $3.00 \pm 1.88$ & $1.00 \pm 0.61$ & $0.49 \pm 0.16$ & $0.54 \pm 0.18$ \\
\hline VEGF & $2.68 \pm 0.17$ & $0.76 \pm 0.07$ & $0.39 \pm 0.05$ & $1.51 \pm 0.62$ & $4.02 \pm 1.17^{\#, \dagger, @}$ & $4.21 \pm 1.02^{\#, \dagger, @}$ \\
\hline
\end{tabular}




\section{Table 3.5 (continued)}

Mean fluorescent intensity of blanks, standards, and samples was measured in duplicate wells, using the Luminex 200 reader (Luminex Corp., Austin, TX). The median fluorescent intensity of blanks was subtracted from all readings. Concentration of each cytokine was calculated from a 5-parameter logistic curve obtained from the known concentration of cytokines. All results were normalized with total protein concentration measured from each tissue lysate ( $\mathrm{pg} / 25 \mu \mathrm{g}$ of total protein). B.D.L represented a value below the detectable concentration range of each analyte and was regarded as zero for statistical calculation. Statistical significance was determined by one-way ANOVA followed by Student-Newman-Keuls test (*: comparison with control mice; ${ }^{*}$ : comparison with 3 dpi mice; ${ }^{\dagger}$ : comparison with 10 dpi mice; ${ }^{@}$ : comparison with 21 dpi mice; ${ }^{\uparrow}$ : comparison with 60 dpi mice; * ${ }^{*},{ }^{\dagger},{ }^{\natural}$ and ${ }^{@}:$ p $<0.05$;

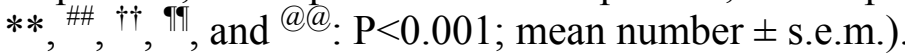


within the brain. This inflammation is characterized by an increase in the number of both resting and activated microglia and differential expression of a number of cytokines, chemokines and growth factors.

Inflammation in the nervous system has been associated with both the initiation and progression of a number of neurological disorders, including Parkinson's disease $(301,302)$. Some of the cardinal pathologies seen in Parkinson's disease include dopaminergic neuronal death in the substantia nigra pars compacta and other regions of the brain, aggregation of ubiquitinated proteins including alpha-synuclein (Lewy bodies) throughout the brain and generalized increases in cerebral oxidative stress (303). Coincident with these processes, there is an activation of the microglia within the brain.

Thus, what remains to be determined is whether the inflammatory reaction seen in the CNS is the outcome of, or initiates these pathologies or both. There is significant research to support each of these possibilities. For example, increases in microglial number, morphology and production of cytokines have been shown to occur in response to overexpression of alpha-synuclein (304-307), death of neurons (308), and increased oxidative stress (309-311). Others have shown that activation of the immune system is likely a predisposing factor that contributes to the initiation and progression of these pathologies (311-314). For example, in rodent PD model, a single systemic injection of inflammogen lipopolysaccharide (LPS) produced an elevation of TNF- $\alpha$ expression and a progressive cell loss in the SN that were sustained for 10 months after injection (315). In addition, mice had been exposed to LPS in prenatal period showed a prolonged inflammatory immune response and a progressive DA neuron loss when they exposed LPS in adulthood, while the prenatal saline control mice did not (316).

In this study, we find that microglia become activated coincident with the physical presence of the H5N1 virus and after this microglial induction we observe loss of the dopaminergic cellular phenotype (atrophy of TH-positive neurons and lack of dopamine production), aggregation of phosphorylated alpha-synuclein (270) and induction of cell death. The rapid microglial activation following peripheral inoculation of the influenza virus raises an important question: Is the initial microglial activation due to the direct sensing of the virus within the brain parenchyma or does it result from signals initiated outside of the brain, i.e. response to induction of a "cytokine storm" in lung or gut?

Support for a direct effect comes from studies demonstrating that both microglia (317) and neurons (318) are capable of directly interacting with the HA protein on the surface of the influenza virus by binding to sialic acid (SA)-alpha 2,3-galactose receptors, where they can induce inflammatory cytokine production $(317,319)$. Other studies, including studies of pandemic $1918 \mathrm{H} 1 \mathrm{~N} 1$ influenza, lend support for an indirect adaptive mechanism which would involve a humoral microglial interaction $(320,321)$.

Based on previous findings showing the physical presence of influenza virus in the brain and probable interactions with circulating T-cells $(322,323)$, we suggest that a combination of mechanisms occur. We find that cytokine expression in the brain, a 
measure of immune activation/response, in most cases closely follows a pattern initiated in lung including IL-6, IL-12, G-CSF, IFN- $\gamma, \mathrm{KC}, \mathrm{MIP}-1 \alpha$, and TNF- $\alpha$ and GM-CSF (with a delayed response), and thus are likely to be, at least in part, initiated in from a lung-initiated cytokine storm.

However, there are clear examples where we find cytokine expression in the brain that have no apparent relationship to the response in lung. IL-13 in the substantia nigra and IL-2 in the striatum were particularly interesting as they showed a rapid induction followed by return to baseline levels and then a reinduction long after $\mathrm{H} 5 \mathrm{~N} 1$ virus is absent from the brain.

IL-13 is originally known as an anti-inflammatory cytokine that suppresses TNF$\alpha$, IL-1 $\beta$, and IL-6 production in activated microglia and inhibits MHC class II and CD4 receptor expression in macrophage both in vivo and in vitro (324-328). However, the proinflammatory effects of IL-13 also have been documented. Microglia derived IL-13 stimulates nicotinamide adenine dinucleotide phosphate (NADPH) oxidase, a major source for reactive oxygen species (ROS) in microglia, and contributes to the neurodegeneration observed in thrombin-treated hippocampus. Neutralization of IL-13, mediated by anti-IL-13 antibodies attenuates the production of inducible nitric oxide synthase (iNOS) and pro-inflammatory cytokines (329). IL-2 is a potent mediator of inflammatory immune response and expressed in response to insults $(330,331)$ and related with a progressive neuronal loss in rodent PD model (329, 332-335).

In our study, the stimulus for reinduction of this cytokines is unknown. However, we note that the timing of cytokine re-induction is coincident with the increased production of dopamine in both the substantia nigra and striatum. Previous studies examining the effects of catecholamine expression on immune activation have shown that dopamine, as an inducer of oxidative stress via quinone formation (310, 336-339), can activate microglia, leading to progressive dopaminergic cell dysfunction and loss (336).

In conclusion, we have found that influenza infection, although resolved within 3 weeks of infection, can instigate a long-term activation of microglia with cytokine production. Since activated microglia function in surveillance and response to insult, their continued presence in certain regions of the brain could make these regions particularly vulnerable to later insults or even normal age-induced changes, which could exacerbate a response that would not normally occur $(340,341)$. Specifically, one could hypothesize that influenza, which does not directly damage the basal ganglia enough to lead to induction of a full-blown disease state could make the basal ganglia sensitive to another insult that would in and of itself also be non-pathogenic. In this manner, it can be proposed that idiopathic Parkinson's disease may result from multiple sub-toxic "hits" (including neurotropic influenza) that occur over the course of a lifetime. 


\section{CHAPTER 4. PRIOR H5N1 INFLUENZA VIRUS INFECTION CONFERS PROTECTION FROM PARAQUAT-INDUCED TOXICITY IN MICE}

\subsection{Introduction}

The "multiple hit" hypothesis suggests that Parkinson's disease may be caused by the combination and/or accumulation of multiple insults to the brain throughout life where the initial event that initiates or contributes to the disease process may occur far earlier than the actual onset of symptoms (342). Mice exposed to environmental toxins, such as paraquat (PQ), during the postnatal period have been shown to become more vulnerable to neurotoxins given in adulthood (74). For example, rats exposed to lipopolysaccharide (LPS) at prenatal stages become more sensitive to 6-hydroxydopamine (6-OHDA) as adults (75). These studies support the concept of a "multiple hit" hypothesis.

Previously, we have shown that acute H5N1 influenza infection can provoke a long-term activation of microglia with specific patterns of cytokine production (Chapter 3 ). This lead us to hypothesize that pre-existing neuroinflammation in certain regions, including the substantia nigra could make these regions vulnerable to or increasingly sensitive to later insults that might not occur without the earlier exposure $(313,341)$.

Therefore, to determine if prior H5N1 virus infection increased the sensitivity to PQ we intranasally infected C57BL/6J mice with $\mathrm{H} 5 \mathrm{~N} 1$ virus. We included mice in the study that showed at least $15 \%$ total body weight loss following H5N1 infection (as an indication that these animals were infected (343)) and administered paraquat-HCl when these animal recovered (60 days post infection). We found that early exposure to H5N1 did not increase the sensitivity of C57BL/6J mice to intraperitoneal (ip) administration of paraquat.

\subsection{Materials and Methods}

All experiment using infectious pathogenic H5N1 viruses, including animal infection, were conducted in a Biosafety level 3+ laboratory approved for use by the U.S. Department of Agriculture and the Center for Disease Control and exclusively utilized by the Division of Virology and other approved scientists at St Jude Children's Research Hospital for highly pathogenic avian influenza work.

\subsubsection{Virus stock preparation and inoculation of mice with H5N1}

Stock viruses were prepared by propagating neurotropic A/VN/1203/04 (H5N1) allantoic cavity of 10-days-old embryonated chicken eggs for 40 to 48 hours at $37^{\circ} \mathrm{C}$. Virus stock was aliquoted then stored at $-70^{\circ} \mathrm{C}$ until use. Viral infectious titers were determined using the method of Reed and Muench (271) and expressed in log10 of the 
$50 \%$ egg infectious dose per $1.0 \mathrm{ml}$ of fluid $\left(\mathrm{EID}_{50} / \mathrm{mL}\right) .6-8$ week old C57BL/6J female mice (Jackson Labs, Bar Harbor, ME) were anesthetized by isofluorane inhalation and infected intranasally with $30 \mu \mathrm{L}$ of allantoic fluid diluted in PBS to the target virus infectious titer $\left(10^{2} \mathrm{EID}_{50}\right)$. One group of animal received $0.9 \%$ saline and was used as an age-matched negative control. The body weight of infected animals was monitored recorded daily.

\subsubsection{Paraquat $(P Q)$ administration}

Paraquat $\mathrm{HCl}$ was purchased from Sigma (856177, Sigma, St. Louis, MO) and diluted with sterilized saline $(0.9 \%)$ to the working concentration of $1 \mathrm{mg} / \mathrm{ml}$. Mice that survived acute H5N1 infection with loss of -at least $-15 \%$ total body weight followed by recovery, were divided into two groups. The first group was administered with ip injection of $5 \mathrm{mg} / \mathrm{kg}$ of paraquat, every third day for three weeks, starting from 60 days post infection. The second group was treated with ip saline (0.9\%). Two control groups were used for comparison. The first control group was aged matched, non-infected C57BL/6J mice administered paraquat using the same injection paradigm as experimental animals to ensure that the paraquat caused a lesion. The second control group was aged matched, non-infected $\mathrm{C} 57 \mathrm{BL} / 6 \mathrm{~J}$ mice administered ip saline to ensure that handling and injection were not a variable in paraquat-induced neurological effects.

\subsubsection{Immunocytochemistry}

One week after the final injection of PQ, mice were deeply anesthetized with Avertin and transcardially perfused with $0.9 \%$ saline followed by $10 \%$ neutral buffered formalin. Brains were removed and postfixed for 3 weeks in $10 \%$ neutral buffered formalin to ensure that any virus particles present in the tissue had been killed. Brains were cryoprotected with $30 \%$ sucrose in PBS, serially sectioned in the coronal plane at 40 micro sections and placed in PBS filled-24 well plates. Sections were immunolabeled for TH (rabbit, P40101-0, PelFreeze, Rogers, AK, 1:500) overnight, and the primary antibody was visualized using the $\mathrm{ABC}$ method (PK-6102, Vector Laboratories, Burlingame, CA) and 3,3-diaminobenzidine (SK-4100 Vector Laboratories, Burlingame, CA) as a chromogen. Sections were mounted on Superfrost-Plus (12-550, Fisher, Pittsburgh, PA) slide and counter stained with neutral red to visualize Nissl substance, and then dehydrated, cleared and mounted with Permount (SP15-500, Sigma, St. Louis, $\mathrm{MO})$.

\subsubsection{Stereological cell count using the optical fractionator method}

The total number of TH-positive dopaminergic neurons in both hemispheres of SNpc was estimated by the optical fractionator method (279) using the StereoInvestigator software (version 7.0, MicroBrightField, Colchester, VT). The broad outlines of the SNpc in both hemispheres were delineated at low power (4× magnification). An unbiased 
counting frame $(60 \times 60 \mu \mathrm{m})$ was placed at the intersections of a grid (frame size $200 \times$ $200 \mu \mathrm{m})$ randomly superimposed on a video image of the contoured sections. Sections were examined under a high power $(100 \times$ objective lens, numerical aperture $(N A)=1.3)$ on a BX51 microscope (Olympus, Center Valley, PA) with a MAC5000 motorized XYZ axis computer-controlled stage and a CX9000 CCD video camera (MicroBrightField, Colchester, VT). TH-positive cells were counted at the depth that their nucleus was focused in each counting area. The reliability of the estimates were measured by calculation of the coefficient of error (344). Gundersen coefficients of error for $\mathrm{m}=1$ was all less than or equal to 0.10 . Statistical significance was calculated using a one-way ANOVA followed by Student-Newman-Keuls post hoc test (280).

\subsubsection{Biochemical measurement of monoamine neurotransmitters}

One week after the last injection of PQ or saline, C57BL/6J mice were deeply anesthetized with Avertin and transcardially perfused with $0.9 \%$ saline to remove the majority of the blood from the brain vasculature. Brains were rapidly removed and placed in a brain matrix (BS-AL-5000C, Braintree Scientific, Braintree, MA) and sliced into 1 $\mathrm{mm}$ thick sections and placed on an ice-cooled plate. Tissues were dissected from the SN (Bregma: -2.70 to -3.70 ), striatum (Bregma: +0.14 to $+1.26 \mathrm{~mm}$ ), brainstem (Bregma: 5.40 to $-6.70 \mathrm{~mm}$ ), cortex (Bregma: -1.70 to $-2.70 \mathrm{~mm}$ ), and hippocampus (Bregma: -1.70 to $-2.70 \mathrm{~mm}$ ) (273). Tissues were homogenized in a chilled $0.3 \mathrm{M}$ perchloric acid and centrifuged for 15 minutes at $10,000 \mathrm{~g}$ at $4^{\circ} \mathrm{C}$. The supernatants were saved and stored at $70^{\circ} \mathrm{C}$ until used. A variety of monoamine transmitters; dopamine (DA), and its metabolites 3,4-dihydroxyphenylacetic acid (DOPAC), and homovanillic acid (HVA); norepinephrine (NE); 5-hydroxytryptamine (5-HT) and its metabolites 5hydroxyindoleacetic acid (5-HIAA) were analyzed using reverse-phase ion pairing HPLC, combined with electrochemical detection (EC) detection under isocratic elution conditions. The amount of monoamine neurotransmitters in the tissues was determined by injecting known concentration of monoamine neurotransmitters and extrapolating from a standard curve. Statistical difference was determined using a one-way ANOVA followed by Student-Newman-Keuls post hoc test

\subsubsection{Quantification of cytokines, chemokines and growth factors}

The concentration of IL-1 $\alpha$, IL-1 $\beta$, IL-2, IL-6, IL-9, IL-10, IL-12(p70), IL-13, TNF- $\alpha$, IFN- $\gamma$, GM-CSF, G-CSF, M-CSF, Eotaxin, IP-10, KC, MCP-1, MIP-1 $\alpha$, MIP-1 $\beta$ and VEGF proteins was simultaneously analyzed from dissected brain regions and lung, using the Luminex 200 system (Luminex Corp., Austin, TX) and the Milliplex mouse cytokine kit (MPXMCYTO-70K-20, Millipore, Billerica, MA).

One week after the last injection of PQ or saline, dissected tissues were homogenized in a buffer containing $50 \mathrm{mM}$ Tris- $\mathrm{HCl}(\mathrm{pH} 7.4), 2.5 \mathrm{mM}$ EDTA, $0.1 \%$ Triton X-100, 150mM NaCl, protease and phosphatase inhibitor cocktail (Complete mini, PhosphoStop, Roche, Indianapolis, IN). The tissues lysates were then incubated for 30 
minutes on ice and centrifuged at $12,000 \mathrm{~g}$ for 15 minutes. Supernatants were aliquoted and stored at $-70^{\circ} \mathrm{C}$ until used. To quantify cytokines, the supernatants were incubated with the microspheres coated with capture antibodies for each analyte for 2 hours. After rinsing, biotinylated detection antibodies were added into each well and incubated for 1 hour. Streptavidin-phycoerythrin conjugates were added and fluorescence intensity was measured by the Luminex 200 reader (Luminex Corp., Austin, TX). Concentration of each cytokine was calculated from a 5-parameter logistic curve obtained from the known concentration of cytokines, using MILLIPLEX Analyst software (Millipore, Billerica, MA, USA).

\subsection{Results}

\subsubsection{Does H5N1 infection synergize with $P Q$ to alter dopaminergic neuron number in the SNpc?}

We assessed, using stereological methods (280), the number of tyrosine hydroxylase positive dopaminergic neurons in the SNpc (Fig 4.1A). We found that $5 \mathrm{mg} / \mathrm{kg}$ PQ treatment reduced the numbers of TH-positive dopaminergic neurons in $\mathrm{SNpc}$ in mice by $22 \%$. Examination of the effect of H5N1 on TH-positive dopaminergic neuron number revealed no significant reduction compared to non-infected saline treated control.

To examine if prior infection with $\mathrm{H} 5 \mathrm{~N} 1$ sensitized C57BL/6J mice to the effects of paraquat, we injected $5 \mathrm{mg} / \mathrm{kg}$ paraquat (using the 3 weeks injection paradigm) into mice that survived acute $\mathrm{H} 5 \mathrm{~N} 1$ infection with loss of -at least $-15 \%$ total body weight followed by recovery, at 60 days after inoculation. The animals treated with paraquat after $\mathrm{H} 5 \mathrm{~N} 1$ infection showed a non-significant $10 \%$ reduction in the number of $\mathrm{TH}-$ positive dopaminergic neurons, compared to non-infected saline treated control animals; a number that was approximately the same as the number determined in $\mathrm{H} 5 \mathrm{~N} 1+$ saline treated mice. These findings suggest that $\mathrm{H} 5 \mathrm{~N} 1$ infection, rather than being a predisposing factor in the reduction of SNpc DA neurons, may in fact be protective.

\subsubsection{Does prior infection with H5N1 synergize with $P Q$ to effect changes in dopamine, DOPAC and HVA levels in the CNS?}

Reverse phase HPLC with electrochemical detection was used to determine if exposure to $\mathrm{H} 5 \mathrm{~N} 1$ infection affected the levels of dopamine and its metabolites DOPAC and HVA in five regions of the CNS: striatum, substantia nigra, brainstem, hippocampus and cortex, and to determine if prior H5N1 exposure modified the levels of these neurochemicals observed following 3 weeks of $5 \mathrm{mg} / \mathrm{kg}$ paraquat.

Although $5 \mathrm{mg} / \mathrm{kg}$ PQ treatment alone significantly decreased the number of THpositive dopaminergic neurons in the SNpc (Fig 4.1), we found that these concentrations 


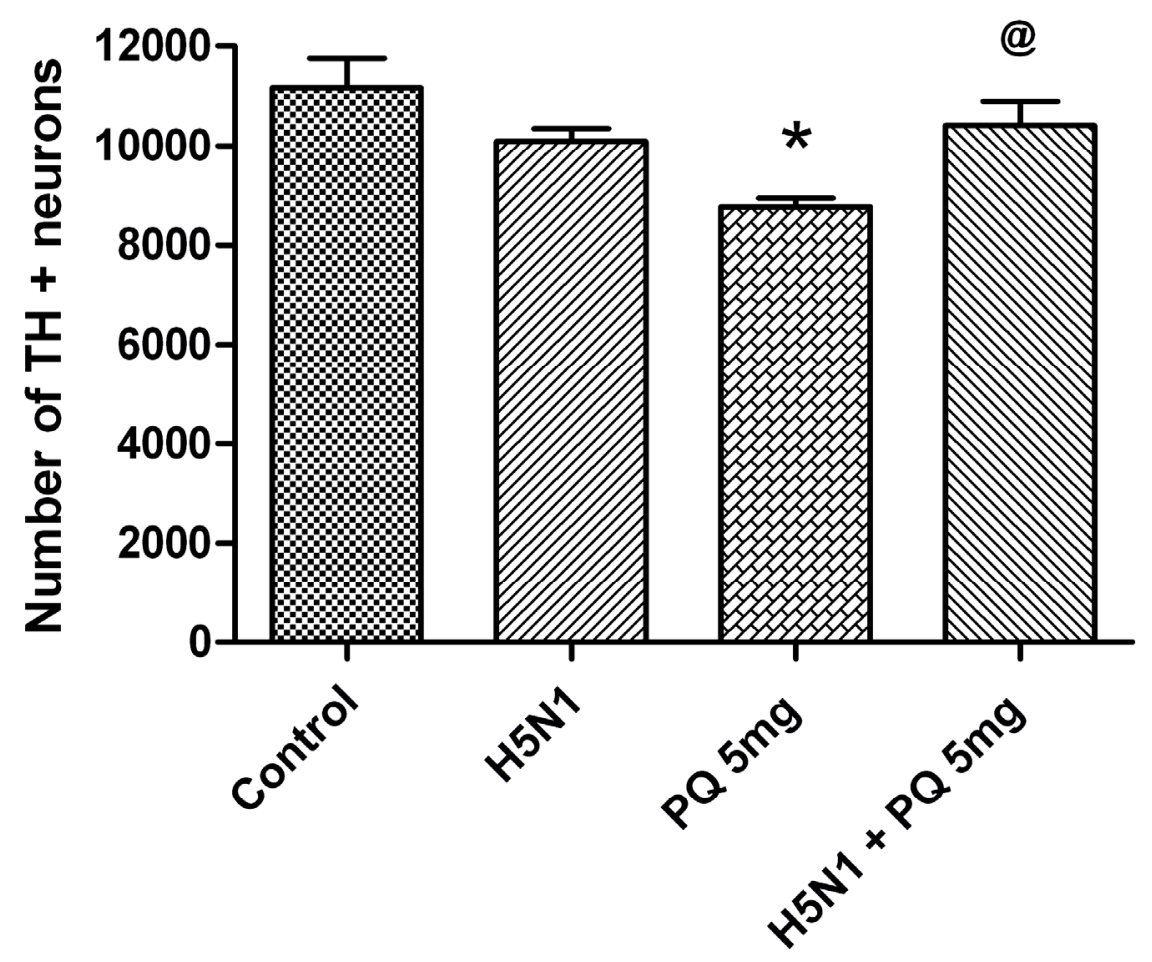

Figure 4.1 Change in the Number of TH-Positive Dopaminergic Neurons Following H5N1 Infection and PQ Treatment

Prior H5N1 infection did not potentiate PQ-induced neurotoxicity to dopaminergic neuron. $5 \mathrm{mg} / \mathrm{kg}$ PQ treatment alone reduced TH-positive SNpc dopaminergic neurons in C57BL/6J mice by $22 \%(8768 \pm 172, \mathrm{n}=5)$ compared to non-infected saline treated control animals $(11170 \pm 599, \mathrm{n}=4)$. Examination of the effect of $\mathrm{H} 5 \mathrm{~N} 1$ on $\mathrm{TH}$-positive dopaminergic neurons $(10090 \pm 259, \mathrm{n}=4)$ revealed no significant change compared to non-infected saline treated controls. Mice received $5 \mathrm{mg} / \mathrm{kg}$ PQ treatment after H5N1 infection showed no change in TH-positive DA neuron number compared to non-infected saline treated control animals $(5 \mathrm{mg} / \mathrm{kg}$ of PQ $+\mathrm{H} 5 \mathrm{~N} 1,10410 \pm 481)$; this number was significantly higher than the number of TH-positive DA neurons in the PQ only treated group and was approximately the same as the number seen in H5N1 only-treated mice. Statistical significance was determined by one-way ANOVA followed by StudentNewman-Keuls test $\left(*\right.$ : comparison with control mice; ${ }^{\#}$ : comparison with H5N1 infected mice; ${ }^{\circledR}$ : comparison with PQ $5 \mathrm{mg} / \mathrm{kg}$ treated mice; ${ }^{*},{ }^{*}$ and ${ }^{\circledR}: \mathrm{p}<0.05 ;{ }^{* *},{ }^{\#}$ and ${ }^{@ @: ~}$ $\mathrm{P}<0.001$; error bars, s.e.m.). 
of PQ did not permanently alter the concentration of DA nor the amount its metabolites DOPAC and HVA in the striatum (Fig 4.2). We also detected no change in the rate of DA turnover, measured as (DOPAC + HVA)/DA, when comparing any of the experimental groups. Similar findings were also seen in substantia nigra (Fig 4.3), brainstem (Fig 4.4), hippocampus (Fig 4.5) and cortex (Fig 4.6). All the experiments examining the predisposing effects of $\mathrm{H} 5 \mathrm{~N} 1$ on PQ-mediated changes were only performed at $5 \mathrm{mg} / \mathrm{kg}$ dose. In this paradigm, H5N1 did not exacerbate the effects of paraquat on dopamine release or turnover in the striatum (Fig 4.2). In the other regions of the brain examined (and that contain significantly lower levels of dopamine), we observed that PQ treatment after H5N1 infection lower total dopamine in the SN compared to DA amount of saline treated control mice (Fig 4.3), while only turnover changes were noted in brainstem (Fig 4.4) and cortex (Fig 4.6).

\subsubsection{Does prior infection with H5N1 synergize with PQ to effect changes in NE, 5-HT and 5-HIAA levels in the CNS?}

Reverse phase HPLC with electrochemical detection was used to determine if prior infection with H5N1 exacerbated paraquat's effects on the level of NE, 5-HT, and 5-HIAA in the SN, striatum, brainstem, cortex, and hippocampus. In the striatum, neither H5N1 nor PQ induced any long-term changes in NE levels. However, both H5N1 infection and paraquat individually reduced the level of 5-HT and 5-HIAA by approximately 50\% compared to basal level. No synergistic effect between the H5N1 infection and paraquat treatment to increase the loss of these monoamines was found (Fig 4.2). Although there were some significant changes to NE, 5-HT and 5HIAA in other regions of the CNS examined (Fig 4.3-4.6), there appeared to be no synergistic effects of $\mathrm{H} 5 \mathrm{~N} 1$ and paraquat.

\subsubsection{Effect of PQ and H5N1 on activation of the immune response in brain}

In the previous chapter, we demonstrated that infection with $\mathrm{H} 5 \mathrm{~N} 1$ led to a longterm increase in the number of resting and activated microglia that reside in the SNpc. We also showed long-term changes in the expression of a number of proinflammatory cytokines, chemokines, and growth factors. This activated immune response implied to speculation that $\mathrm{H} 5 \mathrm{~N} 1$ infection could render the substantia nigra (and perhaps other brain regions) into a heightened state of readiness that could then potentiate the response of the brain to PQ-induced neurotoxicity.

We next examined if the dosage of PQ we used in these studies could alter peripheral cytokine expression levels in the lung, since PQ is a well known pneumotoxin (345). Previous studies have shown that concentrations of PQ ranging from $30 \mathrm{mg} / \mathrm{kg}$ to $120 \mathrm{mg} / \mathrm{kg}$ could induce a significant increase in the level of serum cytokines, including TNF- $\alpha$, IL-1 $\beta$, IL-6, and IL-10 (346). In our studies, much lower concentration of PQ was used since our goal was to establish whether there were synergistic effects between H5N1 and paraquat rather than to study the systemic effects of PQ alone. 

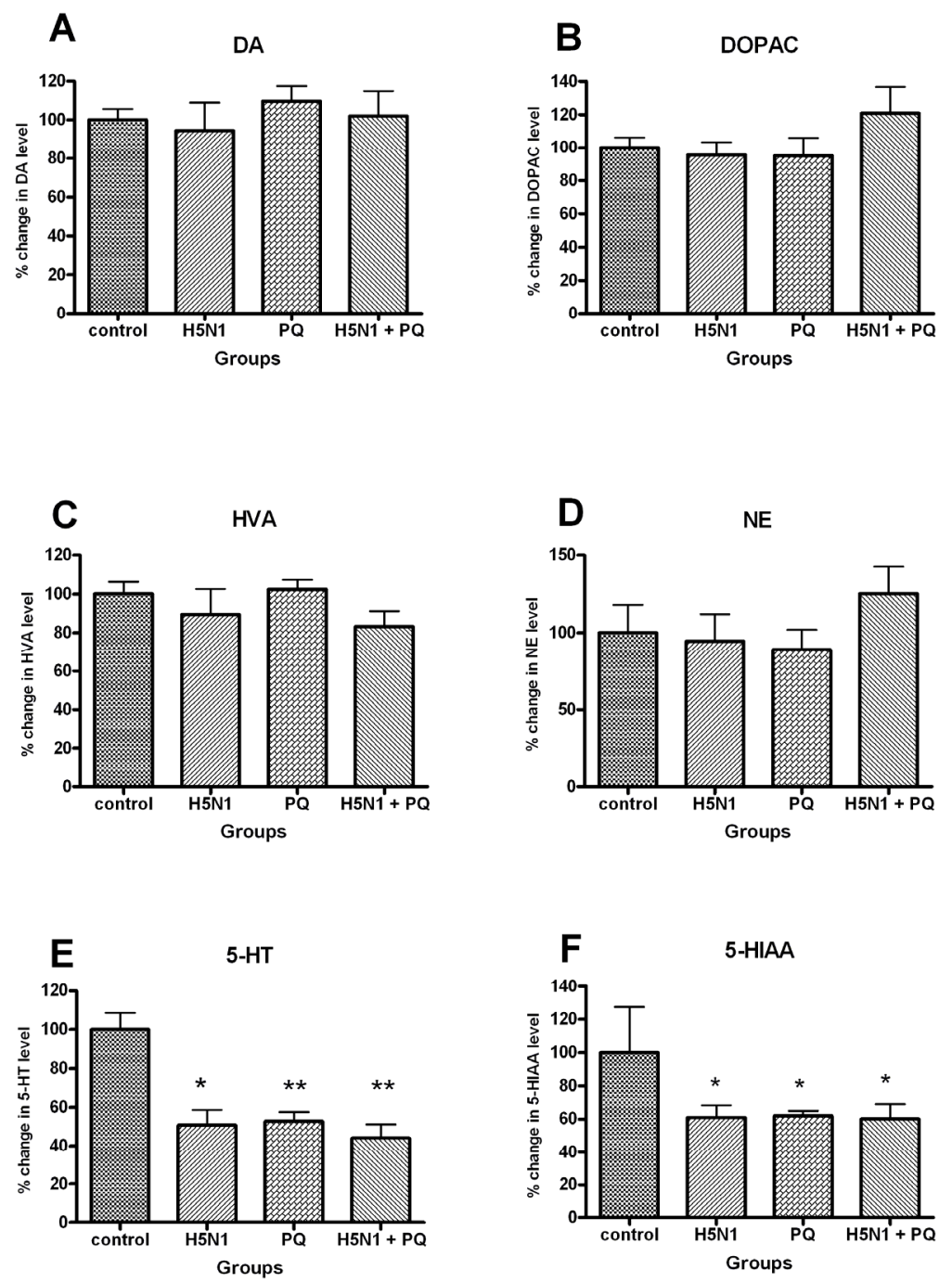

Figure 4.2 Percent Change in Monoamine Neurotransmitter Level in the Striatum Following H5N1 Infection and PQ Treatment

(A-C), No significant change was detected in the amount of striatal DA and its metabolites compared to that of control, regardless of treatments of among H5N1 or PQ alone or combined. (D), DA turnover rate, (DOPAC + HVA)/DA, was conserved throughout all experimental groups. No significant change in the amount of NE was found in any group of animals. (E-F), The amount of 5-HT and 5-HIAA in the H5N1 infected, H5N1+PQ treated, and H5N1+saline treated animals was decreased by $50 \%$ from that of control, but there was no significant difference among the groups. Statistical significance was determined by one-way ANOVA followed by Student-Newman-Keuls test ${ }^{*}$ : comparison with control mice; ${ }^{\#}$ : comparison with $\mathrm{H} 5 \mathrm{~N} 1$ infected mice; ${ }^{\circledR}$ : comparison with PQ 5mg/kg administered mice; * ${ }^{\#}$ and ${ }^{@}: \mathrm{p}<0.05 ; * *,{ }^{\# \#}$ and ${ }^{@}$ : $\mathrm{P}<0.001$; error bars, s.e.m.). 

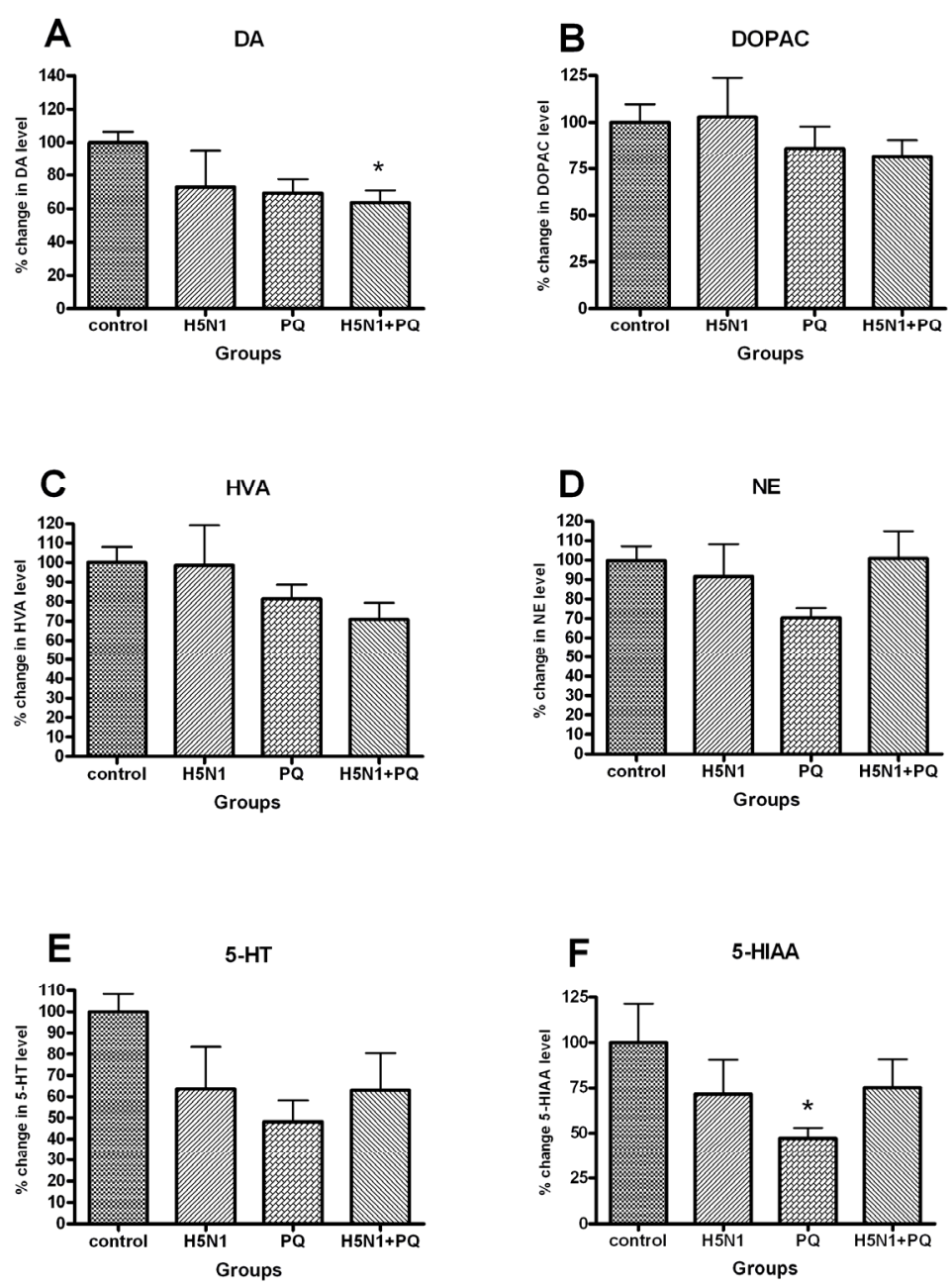

Figure 4.3 Percent Change in Monoamine Neurotransmitter Level in the SN Following H5N1 Infection and PQ Treatment

(A), Mice received PQ after H5N1 infection showed a significant reduction in the level of dopamine in the SN. (B-C), A similar trend was observed in the amounts of DOPAC and HVA, but failed to reach statistical significance. (D-E), No significant change was found in the amount of NE and 5-HT in any group of animals. (F), 5-HIAA was significantly decreased with PQ treatment, compared to basal level in control mice. Statistical significance was determined by one-way ANOVA followed by Student-Newman-Keuls test ${ }^{*}$ : comparison with control mice; ${ }^{\#}$ : comparison with H5N1 infected mice; ${ }^{\circledR}$ : comparison with PQ 5mg/kg administered mice; *, ${ }^{*}$ and ${ }^{@}: \mathrm{p}<0.05 ; * *{ }^{* \#}$ and ${ }^{@ @}$ : $\mathrm{P}<0.001$; error bars, s.e.m.). 

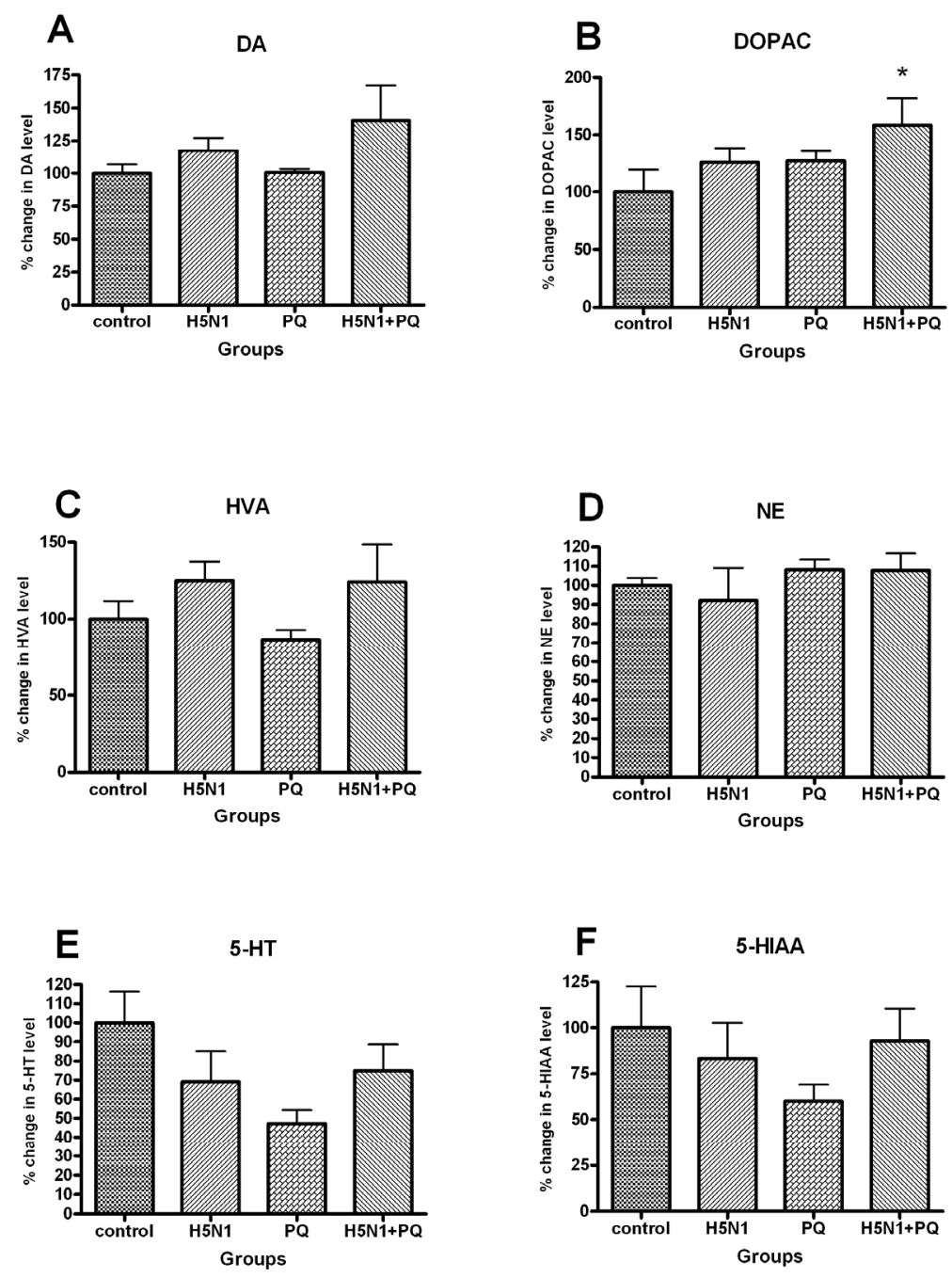

Figure 4.4 Percent Change in Monoamine Neurotransmitter Level in the Brainstem Following H5N1 Infection and PQ Treatment

(A), No additive or synergistic effect between $\mathrm{H} 5 \mathrm{~N} 1$ infection and paraquat treatment was observed in the level of dopamine in the brain stem. (B-C), DOPAC was significantly increased with H5N1+PQ treatment while HVA didn't changed, compared to counterparts in the control mice. (D-F), No significant change was found in the amount of NE, 5-HT, and 5-HIAA in any group of animals. Statistical significance was determined by one-way ANOVA followed by Student-Newman-Keuls test (*: $^{*}$ comparison with control mice; ${ }^{\#}$ : comparison with H5N1 infected mice; ${ }^{@}$ : comparison with PQ 5mg/kg administered mice; *, ${ }^{\#}$ and ${ }^{@}: \mathrm{p}<0.05$; **, ${ }^{\# \#}$ and ${ }^{@ @}: \mathrm{P}<0.001$; error bars, s.e.m.). 

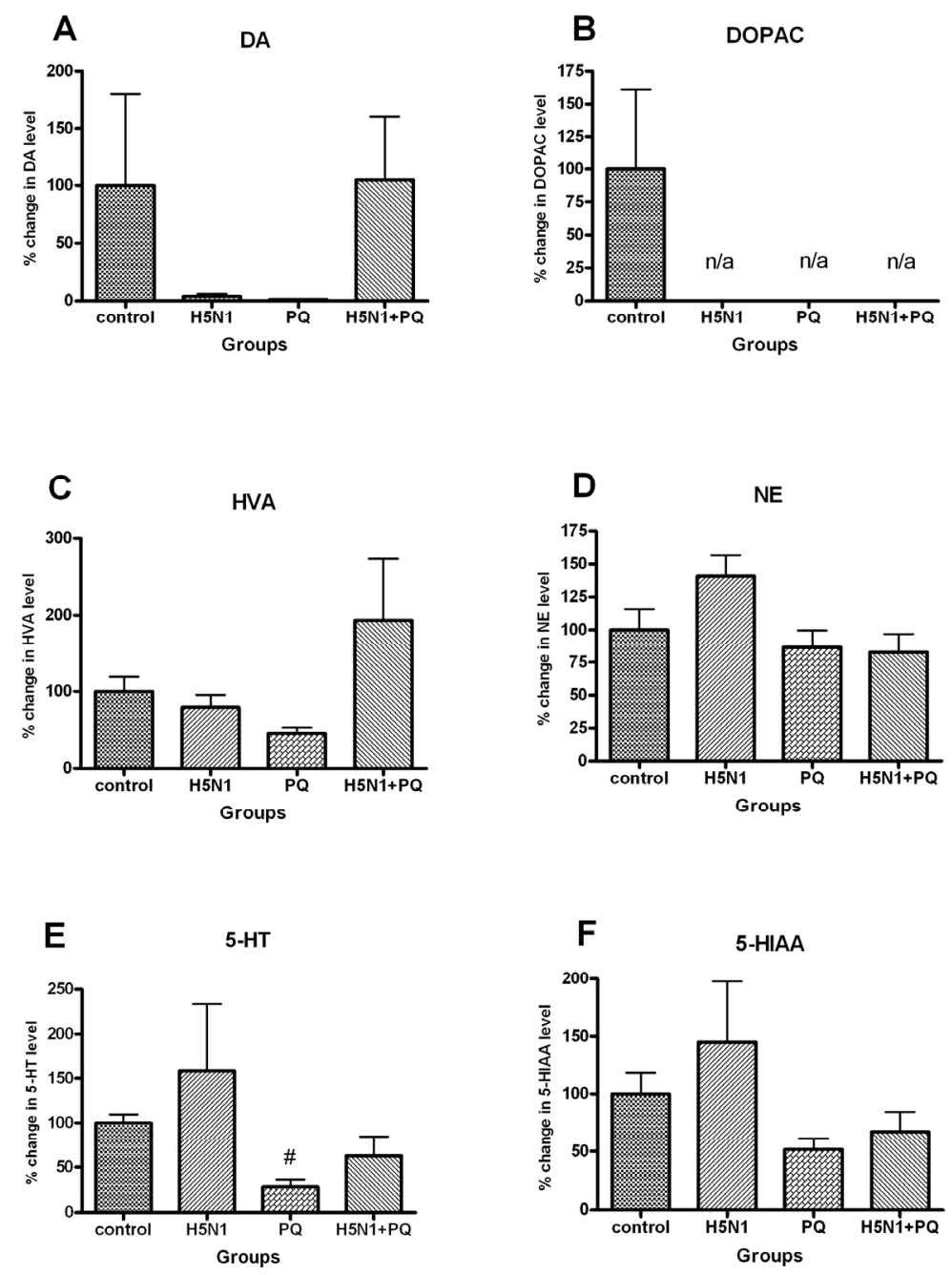

Figure 4.5 Percent Change in Monoamine Neurotransmitter Level in the Hippocampus Following H5N1 Infection and PQ Treatment

(A-D), No additive or synergistic effect between $\mathrm{H} 5 \mathrm{~N} 1$ infection and paraquat treatment was observed in the amount of DA, DOPAC, HVA, and NE in the hippocampus. (E), The amount of 5-HT was significantly decreased in mice that received PQ alone, compared to $\mathrm{H} 5 \mathrm{~N} 1+$ saline treated mice. A similar trend was observed in the amounts of 5-HIAA, but that failed to reach statistical significance. Statistical significance was determined by oneway ANOVA followed by Student-Newman-Keuls test (*: comparison with control mice; \#: comparison with H5N1 infected mice; ${ }^{\circledR}$ : comparison with PQ $5 \mathrm{mg} / \mathrm{kg}$ administered mice; *, ${ }^{\circ}$ and ${ }^{@}: \mathrm{p}<0.05$; **, ${ }^{\#}$ and ${ }^{@ @}: \mathrm{P}<0.001$; error bars, s.e.m.). 
A
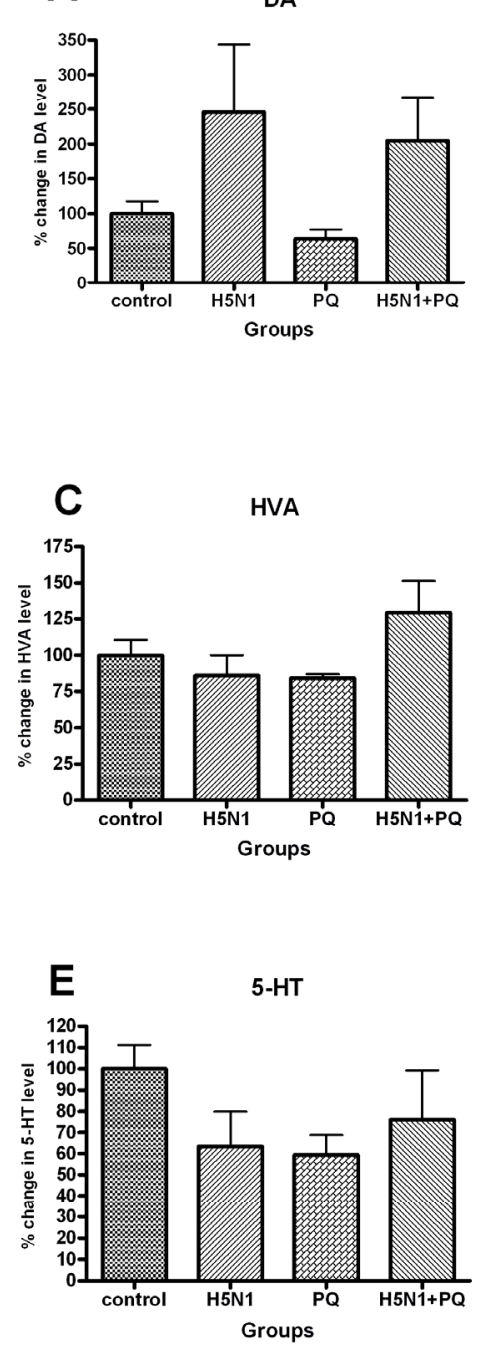
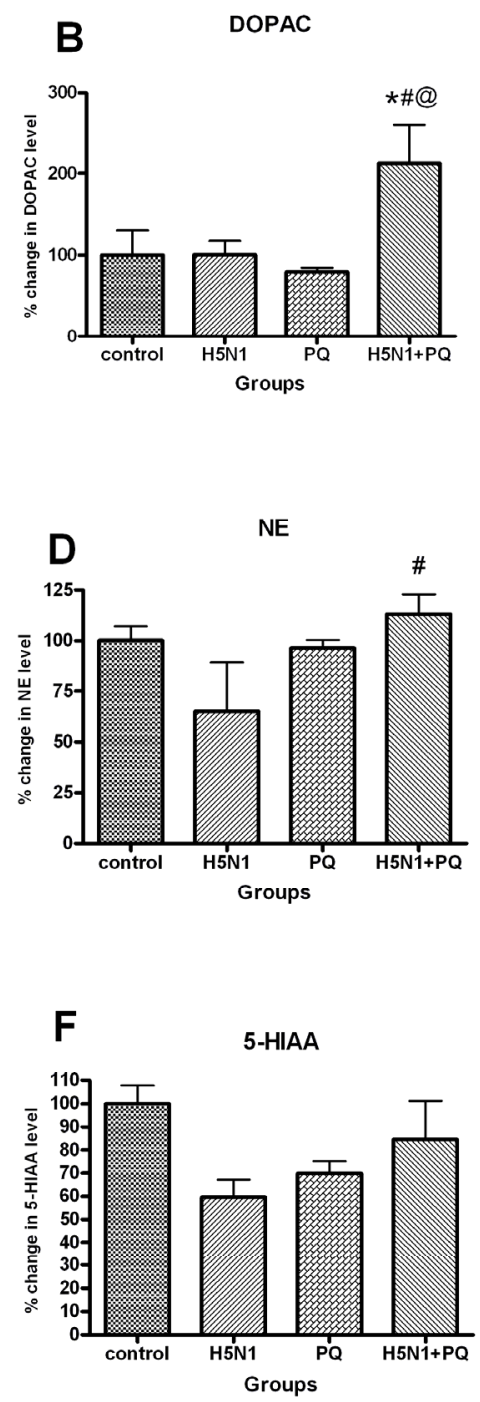

Figure 4.6 Percent Change in Monoamine Neurotransmitter Level in the Cortex Following H5N1 Infection and PQ Treatment

(A), No additive or synergistic effect between $\mathrm{H} 5 \mathrm{~N} 1$ infection and paraquat treatment was observed in the level of DA in the cortex. (B), The level of DOPAC was significantly increased in the H5N1+PQ treated group, compared to the control, H5N1 infected, and PQ alone treated group. (C), A similar trend was observed in the amount of HVA, but that failed to reach statistical significance. (D), The level of NE was significantly increased in the H5N1+PQ treated mice, compared to the H5N1 infected group. (E-F), No significant change was found in the amount of 5-HT and 5-HIAA in any group of animals. Statistical significance was determined by one-way ANOVA followed by

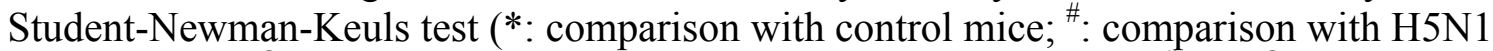
infected mice; ${ }^{\circledR}$ : comparison with PQ $5 \mathrm{mg} / \mathrm{kg}$ administered mice; * ${ }^{\#}$ and ${ }^{\circledR}$ : $\mathrm{p}<0.05 ; * *$, $\#^{\#}$ and ${ }^{\circledR @}$ : $\mathrm{P}<0.001$; error bars, s.e.m.). 
When the lower dose of PQ was administered $(5 \mathrm{mg} / \mathrm{kg})$, a small number of changes in lung cytokine expression were detected, including increases in GM-CSF and IFN- $\gamma$. The levels of each of the other cytokines, chemokines and growth factors examined including IL-1 $\alpha$, IL-1 $\beta$, IL-2, IL-6, IL-9, IL-10, IL-12(p70), Eotaxin, G-CSF, IP-10, KC, M-CSF, MIP-1 $\alpha$, MIP-1 $\beta$, and VEGF were not changed in response to this low level of PQ (Fig 4.7, Table 4.1). To examine if H5N1 infection "primed" the lung to respond to a second challenge we administered $5 \mathrm{mg} / \mathrm{kg}$ PQ at 60 dpi with H5N1. In no cases did we observe synergistic effects of these two agents in any of the cytokines examined. On the contrary, we found that H5N1 priming appeared to dampen the cytokine-mediated immune response, reducing the level of pro-inflammatory cytokines IL-2 and GM-CSF (Fig 4.7, Table 4.1).

In the brain, we found that PQ could induce a specific pattern of cytokines chemokines and growth factors expression dependent on brain region. In the substantia nigra, paraquat significantly increased the level of pro-inflammatory cytokines, chemokines, and growth factors, including IL-2, IL-12(p70), IL-13, Eotaxin, G-CSF, GM-CSF, IFN- $\gamma, \mathrm{KC}, \mathrm{MIP}-1 \alpha$ and MIP- $1 \beta$ compared to cytokine levels measured from the control mice. We found that priming with H5N1 lowered the expression of both pro-, anti-inflammatory mediators including IL-1 $\beta$, IL-2, IL-6, IL-12(p70), IL-13, Eotaxin, GCSF, GM-CSF, IFN- $\gamma$, KC, MCP-1, M-CSF, MIP- $1 \alpha$ and MIP-1 $\beta$, compared to PQ alone treatment without $\mathrm{H} 5 \mathrm{~N} 1$ priming (Fig 4.8, Table 4.2).

In the striatum, administration of the $5 \mathrm{mg} / \mathrm{kg}$ of PQ increased the expression of pro-inflammatory cytokines, chemokines, and growth factors including IL-12(p70), IL-13, GM-CSF, IFN- $\gamma, \mathrm{KC}$, MCP-1, MIP-1 $\alpha$ and MIP-1 $\beta$ (Fig 4.9, Table 4.3). As was observed in the lung and substantia nigra, prior exposure to H5N1 either lowered (IL-13, GM-CSF, KC, MCP-1, MIP-1 $\alpha$ and MIP-1 $\beta$ ) or did not affect (IL-1 $\beta$, IL-2, IL-9, IL-10, IL-12(p70), Eotaxin, IFN- $\gamma$, M-CSF and VEGF) of the inflammatory response to paraquat (Fig 4.9, Table 4.3).

A similar pattern of cytokines/chemokines/growth factors expression was noted in the brainstem (Fig 4.10, Table 4.4) and cortex (Fig 4.11, Table 4.5), suggesting that in the CNS, prior infection with H5N1 either had no effect or reduced PQ-induced immune response.

\subsection{Discussion}

The etiology of the vast majority of Parkinson's disease is unknown. One hypothesis put forward regarding how this disorder is initiated states that there is either additive or synergistic combinatorial effect of different sub-threshold insults that ultimately leads to the development of a parkinsonian pathology. This is the basis for the "multiple hit" hypothesis (70-73). 

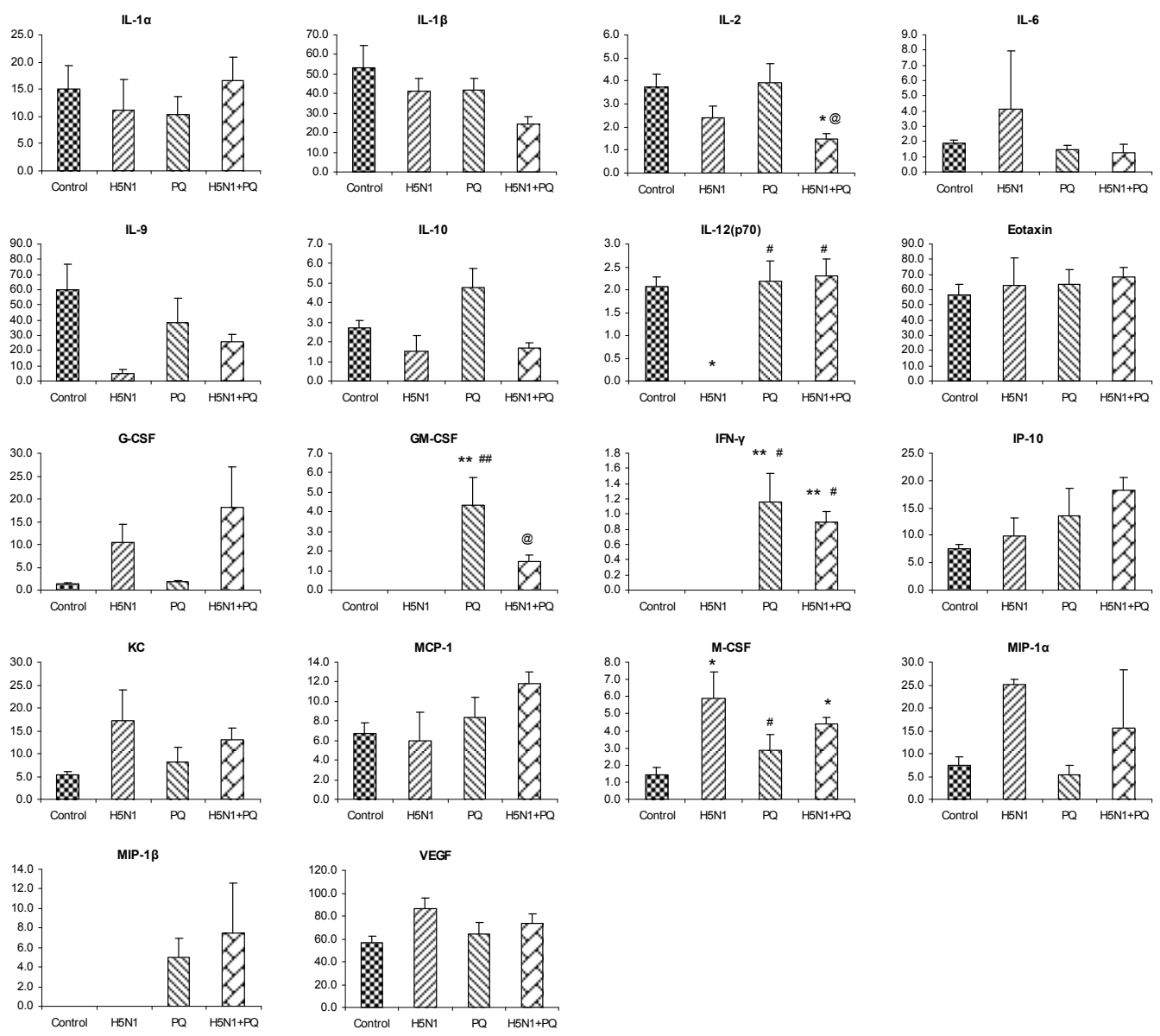

Figure 4.7 Expression of Cytokines, Chemokines and Growth Factors in the Lung Following H5N1 Infection and PQ Treatment

Pro-inflammatory cytokine, IFN- $\gamma$ and growth factors GM-CSF were increased with ip administration of $5 \mathrm{mg} / \mathrm{kg}$ PQ. The rest of the pro-inflammatory mediators examined, including IL-1 $\alpha$, IL-1 $\beta$, IL-2, IL-6, IL-9, IL-13, Eotaxin, G-CSF, IP-10, KC, M-CSF, MCP-1, MIP-1 $\alpha$ and MIP-1 $\beta$ and anti-inflammatory cytokine IL-10 were not significantly different from basal levels in the control, suggesting that 5mg PQ treatment alone did not provoke an massive innate immune response. No synergistic effect between H5N1 infection and PQ treatment to increase the cytokine-mediated immune response was observed. On the contrary, we found that priming with $\mathrm{H} 5 \mathrm{~N} 1$ appeared to dampen the cytokine-mediated immune response after PQ exposure. The level of proinflammatory cytokine IL-2 and growth factor GM-CSF was significantly decreased compared to levels in the PQ alone treated mice. Statistical significance was determined by one-way ANOVA followed by Student-Newman-Keuls test and cytokine levels (pg/25ug of total protein) were presented as mean \pm s.e.m (*: comparison with control mice; ${ }^{\#}$ : comparison with $\mathrm{H} 5 \mathrm{~N} 1$ infected mice; ${ }^{\circledR}$ : comparison with PQ $5 \mathrm{mg} / \mathrm{kg}$ treated mice; * , ${ }^{\text {and }}{ }^{@}: \mathrm{p}<0.05$; **, ${ }^{\#}$ and ${ }^{@ @}$ : $\mathrm{P}<0.001$; error bars, s.e.m.). 
Table 4.1 Level of Cytokines, Chemokines and Growth Factors in the Lung Following H5N1 Infection and PQ Treatment

\begin{tabular}{|c|c|c|c|c|}
\hline Cytokines & Control & H5N1 & PQ & H5N1 + PQ \\
\hline IL- $1 \alpha$ & $14.95 \pm 4.47$ & $11.18 \pm 5.63$ & $10.45 \pm 3.30$ & $16.69 \pm 4.27$ \\
\hline IL-1 $\beta$ & $52.92 \pm 11.44$ & $41.26 \pm 6.63$ & $41.83 \pm 5.99$ & $24.63 \pm 3.67$ \\
\hline IL-2 & $3.73 \pm 0.57$ & $2.41 \pm 0.49$ & $3.93 \pm 0.83$ & $1.47 \pm 0.25 *$,@ \\
\hline IL-9 & $59.77 \pm 16.66$ & $5.14 \pm 2.43$ & $38.61 \pm 15.52$ & $25.52 \pm 5.15$ \\
\hline IL-6 & $1.88 \pm 0.23$ & $4.14 \pm 3.84$ & $1.48 \pm 0.27$ & $1.24 \pm 0.56$ \\
\hline IL-10 & $2.74 \pm 0.38$ & $1.52 \pm 0.83$ & $4.75 \pm 0.99$ & $1.68 \pm 0.29$ \\
\hline IL-12(p70) & $2.06 \pm 0.21$ & B.D.L * & $2.19 \pm 0.44^{\#}$ & $2.31 \pm 0.35^{\#}$ \\
\hline Eotaxin & $56.17 \pm 7.13$ & $62.56 \pm 18.70$ & $63.25 \pm 10.32$ & $68.36 \pm 6.60$ \\
\hline G-CSF & $1.43 \pm 0.25$ & $10.51 \pm 3.92$ & $1.78 \pm 0.42$ & $18.17 \pm 8.82$ \\
\hline GM-CSF & B.D.L & B.D.L & $4.32 \pm 1.41 * *$,\#\# & $1.47 \pm 0.31 @$ \\
\hline IFN- $\gamma$ & B.D.L & B.D.L & $1.16 \pm 0.37 * *, \#$ & $0.89 \pm 0.15 * *, \#$ \\
\hline IP-10 & $7.48 \pm 0.94$ & $9.83 \pm 3.31$ & $13.50 \pm 5.03$ & $18.22 \pm 2.37$ \\
\hline $\mathrm{KC}$ & $5.46 \pm 0.64$ & $17.12 \pm 6.75$ & $8.23 \pm 3.11$ & $12.93 \pm 2.68$ \\
\hline M-CSF & $1.44 \pm 0.43$ & $5.90 \pm 1.54 *$ & $2.82 \pm 0.98^{\#}$ & $4.39 \pm 0.39 *$ \\
\hline MCP-1 & $6.75 \pm 1.11$ & $5.99 \pm 2.94$ & $8.30 \pm 2.08$ & $11.81 \pm 1.24$ \\
\hline MIP-1 $\alpha$ & $7.44 \pm 1.85$ & $25.07 \pm 1.09$ & $5.36 \pm 1.97$ & $15.54 \pm 12.80$ \\
\hline MIP-1 $\beta$ & B.D.L & B.D.L & $5.00 \pm 1.91$ & $7.50 \pm 5.14$ \\
\hline VEGF & $56.96 \pm 5.72$ & $86.88 \pm 9.35$ & $63.83 \pm 10.47$ & $73.85 \pm 7.68$ \\
\hline
\end{tabular}




\section{Table 4.1 (continued)}

Mean fluorescent intensity of blanks, standards, and samples was measured in duplicate wells, using the Luminex 200 reader (Luminex Corp., Austin, TX). The median fluorescent intensity of blanks was subtracted from all readings and concentration of each cytokine was calculated from a 5-parameter logistic curve obtained from the known concentration of cytokines. All results were normalized with total protein concentration measured from each tissue lysate ( $\mathrm{pg} / 25 \mu \mathrm{g}$ of total protein). B.D.L represented a value below the detectable concentration range of each analyte and was regarded as zero for statistical calculation. Statistical significance was determined by one-way ANOVA followed by Student-Newman-Keuls test (*: comparison with control mice; ${ }^{*}$ : comparison with H5N1 infected mice; ${ }^{@}$ : comparison with PQ 5mg/kg treated mice; *, ${ }^{*}$ and ${ }^{@}$ : $\mathrm{p}<0.05 ;{ }^{* *},{ }^{\# \#}$ and ${ }^{@} @$ : P $<0.001$; error bars, s.e.m.). 

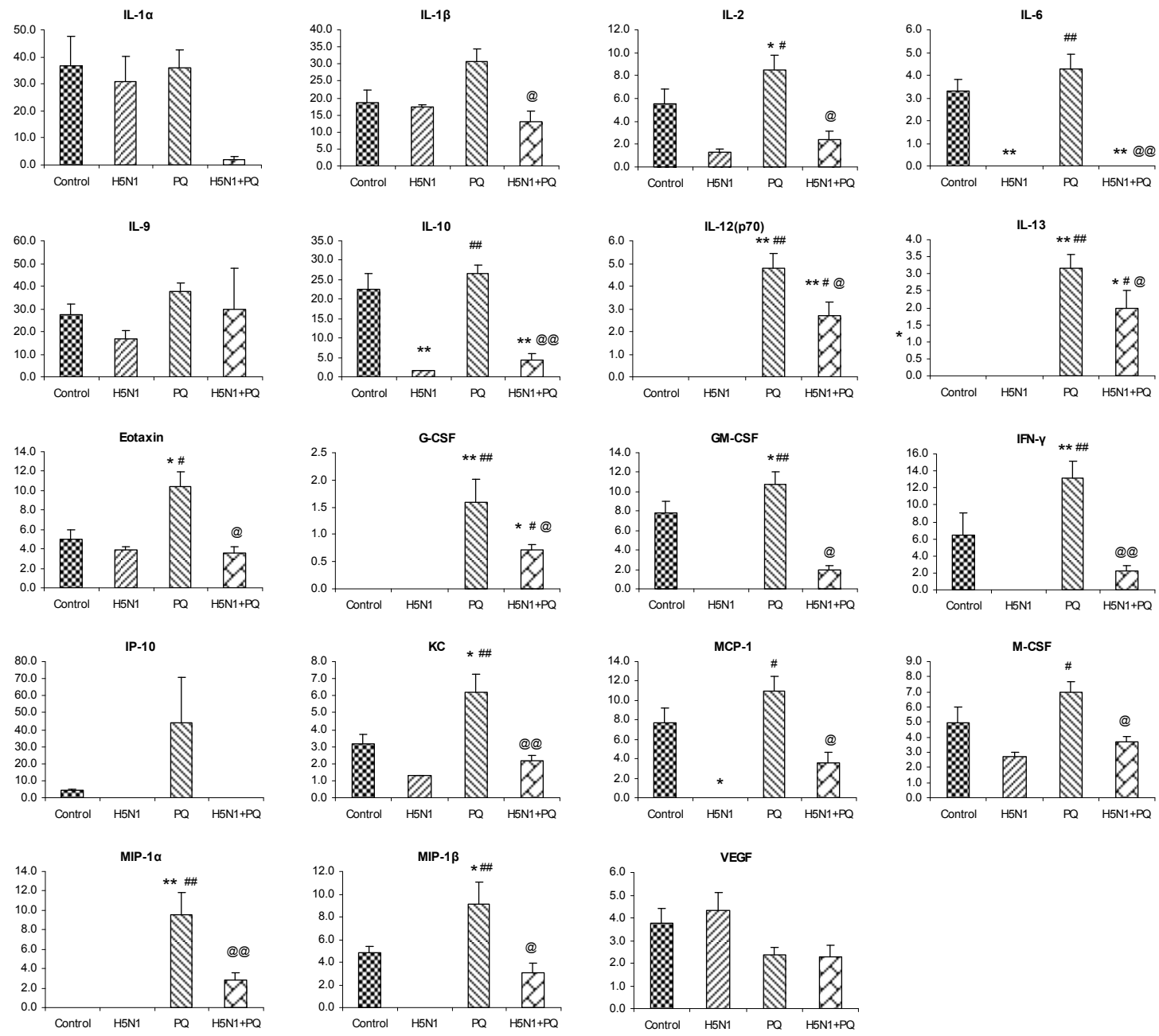

Figure 4.8 Expression of Cytokines, Chemokines and Growth Factors in the SN Following H5N1 Infection and PQ Treatment

Pro-inflammatory cytokines/chemokines/growth factors, including IL-2, IL-12(p70), IL13 , Eotaxin, G-CSF, GM-CSF, IFN- $\gamma$, KC, MIP- $1 \alpha$ and MIP-1 $\beta$ were increased in response to $5 \mathrm{mg}$ PQ treatment, compared to basal levels measured in the control animals. Priming with $\mathrm{H} 5 \mathrm{~N} 1$ resulted in a desensitization and in no cases was there any increases in the inflammatory response. Both the pro- and anti-inflammatory mediators including IL-1 $\beta$, IL-2, IL-6, IL-10, IL-12(p70), IL-13, Eotaxin, G-CSF, GM-CSF, IFN- $\gamma$, KC, MCP-1, M-CSF, MIP-1 $\alpha$ and MIP-1 $\beta$ were decreased in H5N1+PQ groups, compared to PQ alone treated group. Statistical significance was determined by one-way ANOVA followed by Student-Newman-Keuls test and cytokine levels (pg/25ug of total protein) were presented as mean \pm s.e.m (*: comparison with control mice; ${ }^{\#}$ : comparison with H5N1 infected mice; ${ }^{\circledR}$ : comparison with PQ 5mg/kg treated mice; * ${ }^{*}$ and ${ }^{\circledR}: \mathrm{p}<0.05$; **, ${ }^{\#}$ and ${ }^{@ @}$ : $\mathrm{P}<0.001$; error bars, s.e.m.). 
Table 4.2 Level of Cytokines, Chemokines and Growth Factors in the SN Following H5N1 Infection and PQ Treatment

\begin{tabular}{|c|c|c|c|c|}
\hline Cytokines & Control & H5N1 & PQ & H5N1 + PQ \\
\hline IL-1 $\alpha$ & $36.77 \pm 10.73$ & $30.99 \pm 9.27$ & $35.81 \pm 6.74$ & $1.87 \pm 1.30$ \\
\hline IL-1 $\beta$ & $18.54 \pm 3.67$ & $17.27 \pm 0.78$ & $30.73 \pm 3.69$ & $13.12 \pm 2.86^{@}$ \\
\hline IL-2 & $5.56 \pm 1.27$ & $1.28 \pm 0.29$ & $8.50 \pm 1.28 *$ *\# & $2.44 \pm 0.73 @$ \\
\hline IL-6 & $3.31 \pm 0.49$ & B.D.L ** & $4.30 \pm 0.61 \#$ & B.D.L**,@@ \\
\hline IL-9 & $27.48 \pm 4.81$ & $16.79 \pm 3.71$ & $37.74 \pm 3.46$ & $29.58 \pm 18.49$ \\
\hline IL-10 & $22.50 \pm 4.00$ & $1.66 \pm 0.06^{* *}$ & $26.59 \pm 2.12^{\# \#}$ & $4.40 \pm 1.43 * *, @ @$ \\
\hline IL-12(p70) & B.D.L & B.D.L & $4.81 \pm 0.62 * *, \# \#$ & $2.70 \pm 0.61 * *, \#, @$ \\
\hline IL-13 & B.D.L & B.D.L & $3.17 \pm 0.40 * *, \# \#$ & $1.98 \pm 0.53 *$ *,\#,@ \\
\hline Eotaxin & $4.95 \pm 1.02$ & $3.86 \pm 0.34$ & $10.40 \pm 1.56 *, \#$ & $3.57 \pm 0.69 @$ \\
\hline G-CSF & B.D.L & B.D.L & $1.59 \pm 0.42 * *, \# \#$ & $0.71 \pm 0.11 *, \#, @$ \\
\hline GM-CSF & $7.81 \pm 1.25$ & B.D.L & $10.73 \pm 1.30 *$ & $2.00 \pm 0.43 @$ \\
\hline IFN- $\gamma$ & $6.48 \pm 2.55$ & B.D.L & $13.20 \pm 1.89 * *$ \#\# & $2.27 \pm 0.55 @$ \\
\hline IP-10 & $4.24 \pm 1.02$ & B.D.L & $43.97 \pm 26.75$ & B.D.L \\
\hline $\mathrm{KC}$ & $3.16 \pm 0.54$ & $1.27 \pm 0.05$ & $6.20 \pm 1.06^{*, \# \#}$ & $2.16 \pm 0.29 @$ \\
\hline MCP-1 & $7.68 \pm 1.59$ & B.D.L * & $10.91 \pm 1.62^{\#}$ & $3.61 \pm 1.11 @$ \\
\hline M-CSF & $4.99 \pm 1.02$ & $2.75 \pm 0.23$ & $6.94 \pm 0.70^{\#}$ & $3.72 \pm 0.31 @$ \\
\hline MIP-1 $\alpha$ & B.D.L & B.D.L & $9.57 \pm 2.28 * *, \# \#$ & $2.82 \pm 0.81 @$ \\
\hline MIP-1 $\beta$ & $4.79 \pm 0.61$ & B.D.L & $9.10 \pm 1.97 *$ *\# & $3.09 \pm 0.79 @$ \\
\hline VEGF & $3.75 \pm 0.65$ & $4.33 \pm 0.78$ & $2.39 \pm 0.30$ & $2.28 \pm 0.50$ \\
\hline
\end{tabular}




\section{Table 4.2 (continued)}

Mean fluorescent intensity of blanks, standards, and samples was measured in duplicate wells, using the Luminex 200 reader (Luminex Corp., Austin, TX). The median fluorescent intensity of blanks was subtracted from all readings and concentration of each cytokine was calculated from a 5-parameter logistic curve obtained from the known concentration of cytokines. All results were normalized with total protein concentration measured from each tissue lysate ( $\mathrm{pg} / 25 \mu \mathrm{g}$ of total protein). B.D.L represented a value below the detectable concentration range of each analyte and was regarded as zero for statistical calculation. Statistical significance was determined by one-way ANOVA followed by Student-Newman-Keuls test (*: comparison with control mice; ${ }^{*}$ : comparison with H5N1 infected mice; ${ }^{@}$ : comparison with PQ 5mg/kg treated mice; *, ${ }^{*}$ and ${ }^{@}$ : $\mathrm{p}<0.05 ;{ }^{* *},{ }^{\# \#}$ and ${ }^{@} @$ : P $<0.001$; error bars, s.e.m.). 

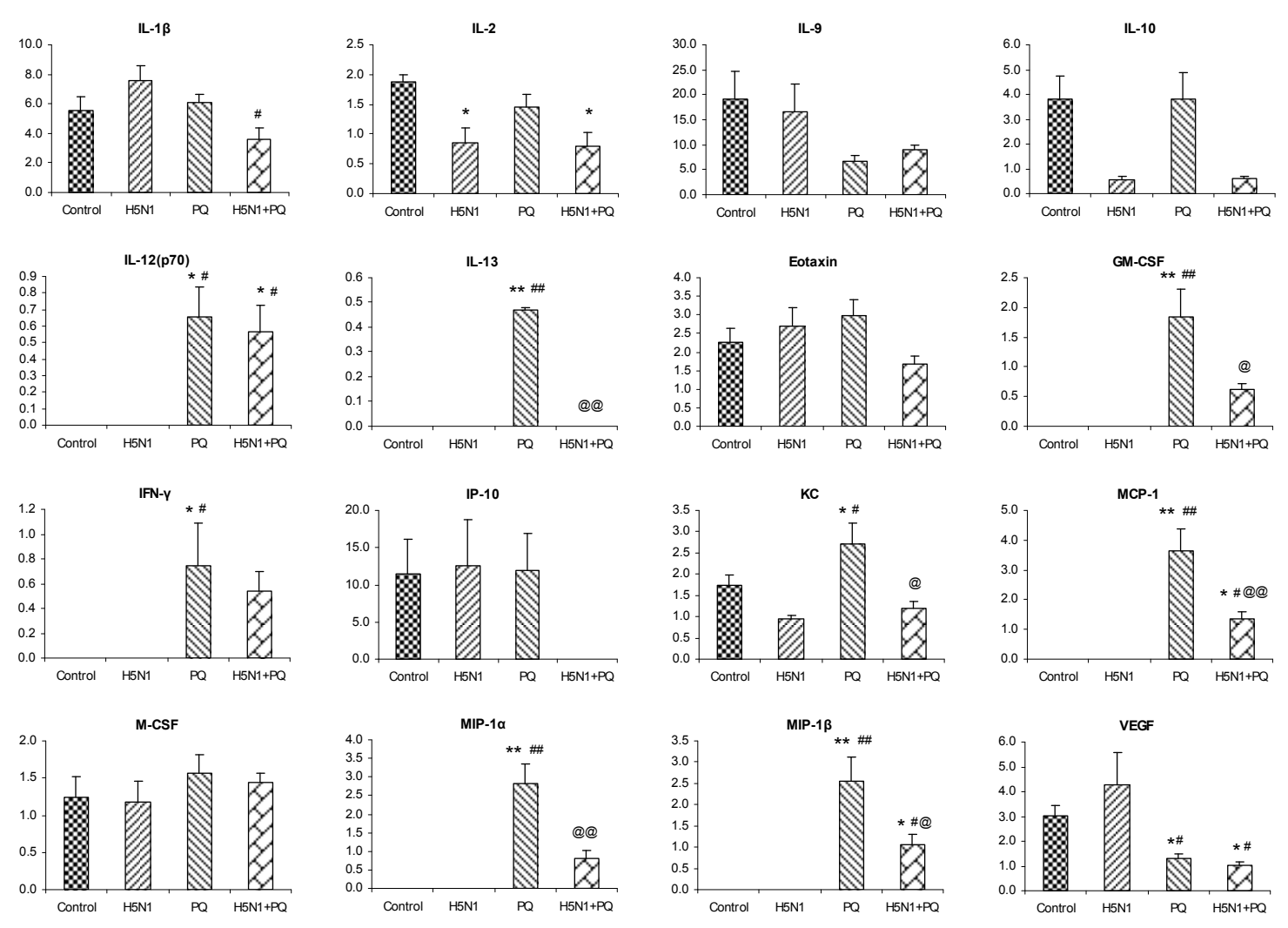

Figure 4.9 Expression of Cytokines, Chemokines and Growth Factors in the Striatum Following H5N1 Infection and PQ Treatment

Pro-inflammatory cytokines/chemokines/growth factors including IL-12(p70), IL-13, GM-CSF, IFN- $\gamma$, KC, MCP-1, MIP-1 $\alpha$ and MIP-1 $\beta$ were increased responds to ip administration of $5 \mathrm{mg} / \mathrm{kg}$ PQ, compared to basal levels measured in the control animals. As was observed in the lung and substantia nigra, prior exposure to H5N1 either lowered (IL-13, GM-CSF, KC, MCP-1, MIP-1 $\alpha$, and MIP-1 $\beta$ ) or did not affect (IL-1 $\beta$, IL-2, IL-9, IL-12(p70), Eotaxin, IFN- $\gamma$, IP-10, M-CSF and VEGF) the inflammatory response to paraquat. Statistical significance was determined by one-way ANOVA followed by Student-Newman-Keuls test and cytokine levels (pg/25ug of total protein) were presented as mean \pm s.e.m (*: comparison with control mice; ${ }^{*}$ : comparison with $\mathrm{H} 5 \mathrm{~N} 1$ infected mice; ${ }^{@}$ : comparison with PQ 5mg/kg treated mice; ${ }^{*},{ }^{\#}$ and ${ }^{@}: \mathrm{p}<0.05 ;{ }^{* *},{ }^{\#}$ and ${ }^{@ @}$ : $\mathrm{P}<0.001$; error bars, s.e.m.). 
Table 4.3 Level of Cytokines, Chemokines and Growth Factors in the Striatum Following H5N1 Infection and PQ Treatment

\begin{tabular}{|c|c|c|c|c|}
\hline Cytokines & Control & H5N1 & $\mathbf{P Q}$ & H5N1 + PQ \\
\hline IL-1 $\beta$ & $5.54 \pm 0.91$ & $7.61 \pm 0.97$ & $6.06 \pm 0.62$ & $3.60 \pm 0.81^{\#}$ \\
\hline IL-2 & $1.88 \pm 0.12$ & $0.84 \pm 0.26 *$ & $1.44 \pm 0.22$ & $0.80 \pm 0.22 *$ \\
\hline IL-9 & $19.10 \pm 5.67$ & $16.58 \pm 5.51$ & $6.68 \pm 1.07$ & $9.05 \pm 0.78$ \\
\hline IL-10 & $3.81 \pm 0.94$ & $0.58 \pm 0.11$ & $3.80 \pm 1.10$ & $0.60 \pm 0.10$ \\
\hline IL-12(p70) & B.D.L & B.D.L & $0.66 \pm 0.18 *, \#$ & $0.57 \pm 0.16^{*, \#}$ \\
\hline IL-13 & B.D.L & B.D.L & $0.47 \pm 0.01 * *, \# \#$ & B.D.L@@ \\
\hline Eotaxin & $2.25 \pm 0.39$ & $2.69 \pm 0.52$ & $2.96 \pm 0.46$ & $1.69 \pm 0.21$ \\
\hline GM-CSF & B.D.L & B.D.L & $1.84 \pm 0.47 * *$ *\# & $0.62 \pm 0.11 @$ \\
\hline IFN- $\gamma$ & B.D.L & B.D.L & $0.74 \pm 0.35 *, \#$ & $0.54 \pm 0.15$ \\
\hline IP-10 & $11.42 \pm 4.73$ & $12.48 \pm 6.23$ & $11.89 \pm 5.03$ & B.D.L \\
\hline $\mathrm{KC}$ & $1.73 \pm 0.26$ & $0.95 \pm 0.07$ & $2.70 \pm 0.51 *, \#$ & $1.21 \pm 0.14^{@}$ \\
\hline M-CSF & $1.24 \pm 0.28$ & $1.18 \pm 0.27$ & $1.57 \pm 0.25$ & $1.45 \pm 0.13$ \\
\hline MCP-1 & B.D.L & B.D.L & $3.65 \pm 0.72 * *$ & $1.37 \pm 0.22$ *,\#,@@ \\
\hline MIP-1 $\alpha$ & B.D.L & B.D.L & $2.82 \pm 0.54 * *$ \#\# & $0.80 \pm 0.22 @$ \\
\hline MIP-1 $\beta$ & B.D.L & B.D.L & $2.55 \pm 0.58 * *$,\#\# & $1.05 \pm 0.25 *, \#, @$ \\
\hline VEGF & $3.02 \pm 0.44$ & $4.29 \pm 1.29$ & $1.32 \pm 0.17^{*, \#}$ & $1.00 \pm 0.15^{*, \#}$ \\
\hline
\end{tabular}




\section{Table 4.3 (continued)}

Mean fluorescent intensity of blanks, standards, and samples was measured in duplicate wells, using the Luminex 200 reader (Luminex Corp., Austin, TX). The median fluorescent intensity of blanks was subtracted from all readings and concentration of each cytokine was calculated from a 5-parameter logistic curve obtained from the known concentration of cytokines. All results were normalized with total protein concentration measured from each tissue lysate ( $\mathrm{pg} / 25 \mu \mathrm{g}$ of total protein). B.D.L represented a value below the detectable concentration range of each analyte and was regarded as zero for statistical calculation. Statistical significance was determined by one-way ANOVA followed by Student-Newman-Keuls test (*: comparison with control mice; ${ }^{*}$ : comparison with H5N1 infected mice; ${ }^{@}$ : comparison with PQ 5mg/kg treated mice; *, ${ }^{*}$ and ${ }^{@}$ : $\mathrm{p}<0.05 ;{ }^{* *},{ }^{\# \#}$ and ${ }^{@} @$ : P $<0.001$; error bars, s.e.m.). 

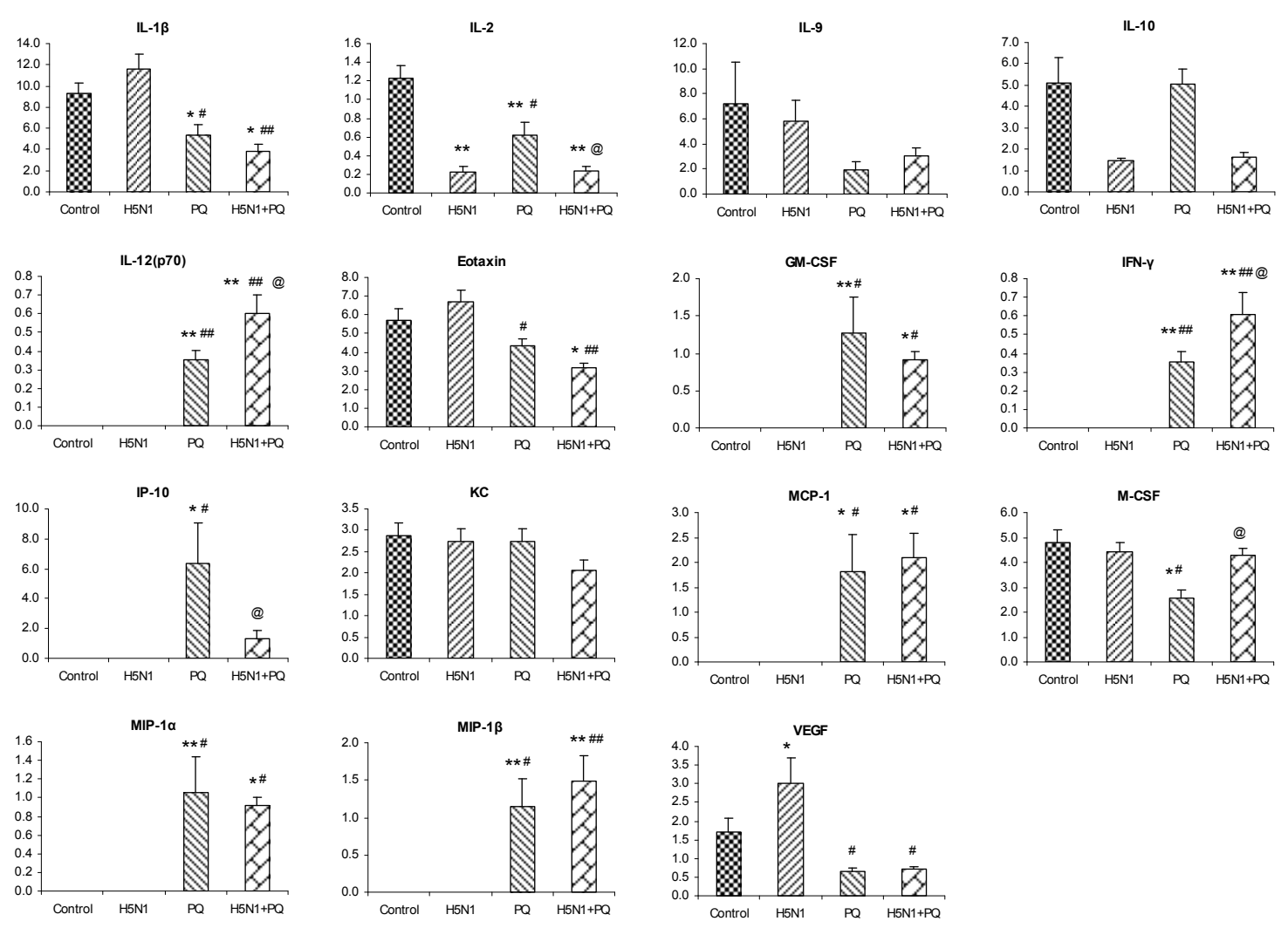

Figure 4.10 Expression of Cytokines, Chemokines and Growth Factors in the Brainstem Following H5N1 Infection and PQ Treatment

Pro-inflammatory cytokines/chemokines/growth factors including IL-12(p70), GM-CSF, IFN- $\gamma$, IP-10, MCP-1, MIP-1 $\alpha$, MIP-1 $\beta$ and VEGF were increased responds to $5 \mathrm{mg}$ PQ treatment, compared to basal levels measured in the control animals. Prior exposure to H5N1 lowered (IL-2 and IP-10) or raised (IL-12(p70), IFN- $\gamma$, and, M-CSF) or did not affect (IL-1 $\beta$, IL-9, IL-10, Eotaxin, GM-CSF, KC, MCP-1, MIP-1 $\alpha$, MIP-1 $\beta$ and VEGF) the inflammatory response to paraquat. Statistical significance was determined by oneway ANOVA followed by Student-Newman-Keuls test and cytokine levels (pg/25ug of total protein) were presented as mean \pm s.e.m (*: comparison with control mice; \#: comparison with H5N1 infected mice; ${ }^{@}$ : comparison with PQ $5 \mathrm{mg} / \mathrm{kg}$ treated mice; * * and ${ }^{@}: \mathrm{p}<0.05 ; * *,{ }^{\#}$ and ${ }^{@ @}: \mathrm{P}<0.001$; error bars, s.e.m.). 
Table 4.4 Level of Cytokines, Chemokines and Growth Factors in the Brainstem Following H5N1 Infection and PQ Treatment

\begin{tabular}{|c|c|c|c|c|}
\hline Cytokines & Control & H5N1 & $\mathbf{P Q}$ & H5N1 + PQ \\
\hline IL-1 $\beta$ & $9.27 \pm 1.05$ & $11.62 \pm 1.35$ & $5.33 \pm 0.98 * \#$ & $3.80 \pm 0.66 * \#$ \\
\hline IL-2 & $1.23 \pm 0.13$ & $0.22 \pm 0.06 * *$ & $0.62 \pm 0.14 * *, \#$ & $0.24 \pm 0.05 * *, @$ \\
\hline IL-9 & $7.23 \pm 3.32$ & $5.82 \pm 1.64$ & $1.97 \pm 0.59$ & $3.08 \pm 0.58$ \\
\hline IL-10 & $5.11 \pm 1.19$ & $1.49 \pm 0.11$ & $5.04 \pm 0.71$ & $1.65 \pm 0.21$ \\
\hline IL-12(p70) & B.D.L & B.D.L & $0.36 \pm 0.05 * *$ & $0.60 \pm 0.10 * *$,\#\#,@ \\
\hline Eotaxin & $5.73 \pm 0.57$ & $6.67 \pm 0.67$ & $4.33 \pm 0.40^{\#}$ & $3.17 \pm 0.22 * \#$ \\
\hline GM-CSF & B.D.L & B.D.L & $1.28 \pm 0.48 * *, \#$ & $0.92 \pm 0.10 * \#$ \\
\hline IFN- $\gamma$ & B.D.L & B.D.L & $0.35 \pm 0.06 * *$ *\#\# & $0.61 \pm 0.11 * *$,\#\#,@ \\
\hline IP-10 & B.D.L & B.D.L & $6.32 \pm 2.74 *, \#$ & $1.34 \pm 0.49^{@}$ \\
\hline $\mathrm{KC}$ & $2.86 \pm 0.30$ & $2.73 \pm 0.30$ & $2.74 \pm 0.29$ & $2.05 \pm 0.24$ \\
\hline MCP-1 & B.D.L & B.D.L & $1.82 \pm 0.73 * \#$ & $2.09 \pm 0.50 *, \#$ \\
\hline $\mathrm{M}-\mathrm{CSF}$ & $4.81 \pm 0.51$ & $4.42 \pm 0.38$ & $2.54 \pm 0.33 * \#$ & $4.27 \pm 0.30^{@}$ \\
\hline MIP-1 $\alpha$ & B.D.L & B.D.L & $1.06 \pm 0.38 * * \#$ & $0.92 \pm 0.09 *, \#$ \\
\hline MIP-1 $\beta$ & B.D.L & B.D.L & $1.15 \pm 0.37 * *, \#$ & $1.49 \pm 0.33 * *$ \#\# \\
\hline VEGF & $1.70 \pm 0.36$ & $3.00 \pm 0.71 *$ & $0.66 \pm 0.07^{\#}$ & $0.70 \pm 0.07^{\#}$ \\
\hline
\end{tabular}




\section{Table 4.4 (continued)}

Mean fluorescent intensity of blanks, standards, and samples was measured in duplicate wells, using the Luminex 200 reader (Luminex Corp., Austin, TX). The median fluorescent intensity of blanks was subtracted from all readings and concentration of each cytokine was calculated from a 5-parameter logistic curve obtained from the known concentration of cytokines. All results were normalized with total protein concentration measured from each tissue lysate ( $\mathrm{pg} / 25 \mu \mathrm{g}$ of total protein). B.D.L represented a value below the detectable concentration range of each analyte and was regarded as zero for statistical calculation. Statistical significance was determined by one-way ANOVA followed by Student-Newman-Keuls test (*: comparison with control mice; ${ }^{*}$ : comparison with H5N1 infected mice; ${ }^{@}$ : comparison with PQ 5mg/kg treated mice; *, ${ }^{*}$ and ${ }^{@}$ : $\mathrm{p}<0.05 ;{ }^{* *},{ }^{\# \#}$ and ${ }^{@} @$ : P $<0.001$; error bars, s.e.m.). 

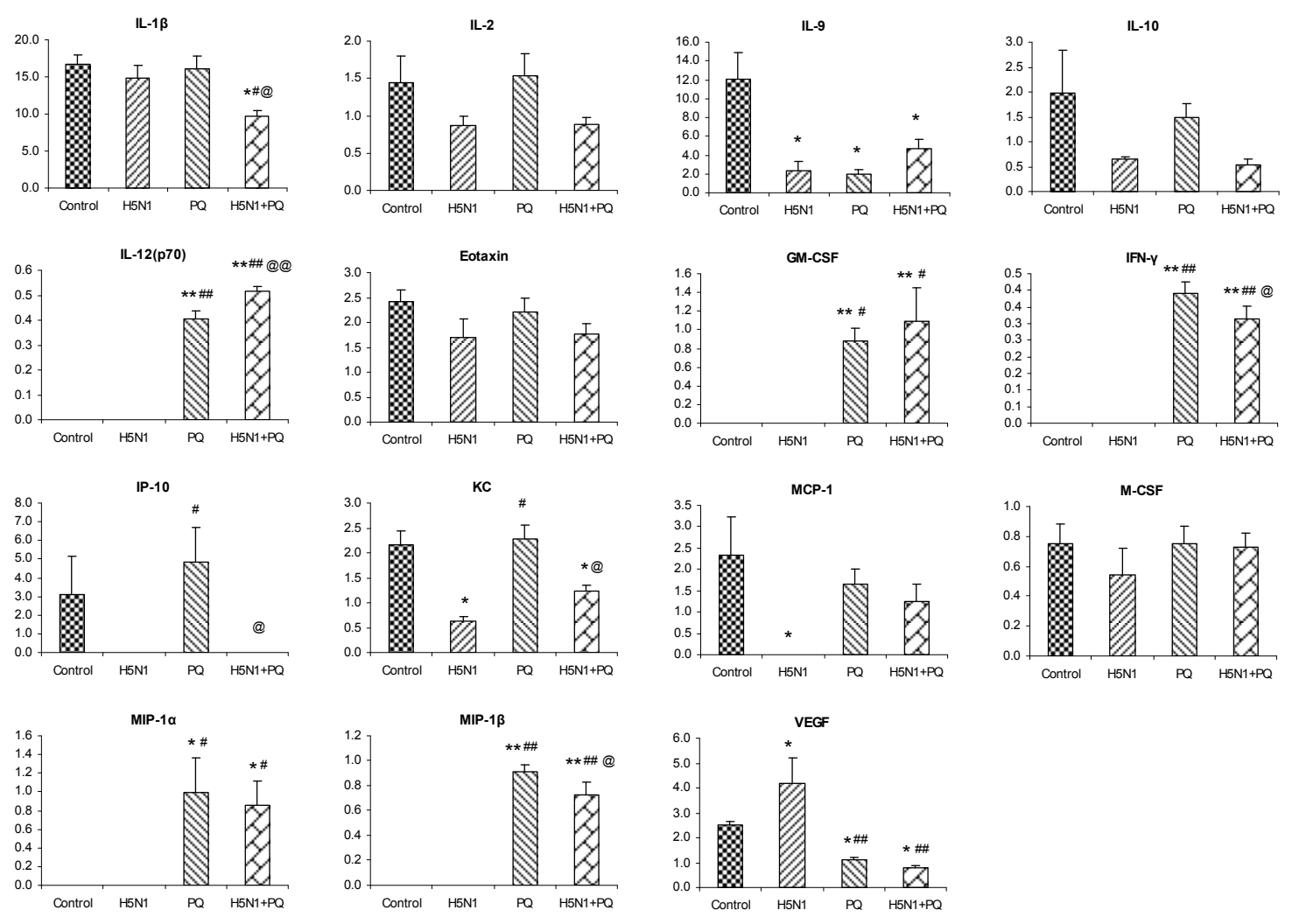

Figure 4.11 Expression of Cytokines, Chemokines and Growth Factors in the Cortex Following H5N1 Infection and PQ Treatment

Pro-inflammatory cytokines including IL-12 (p70), GM-CSF, IFN- $\gamma$, MIP-1 $\alpha$, MIP-1 $\beta$ and VEGF were increased responds to $5 \mathrm{mg}$ PQ treatment, compared to basal levels measured in the control animals. Prior exposure to H5N1 either lowered (IL-1 $\beta$, IFN- $\gamma$, IP-10, KC, MIP-1 $\beta$ ) or did not affect (IL-2, IL-9, IL-10, Eotaxin, GM-CSF, MCP-1, MCSF, MIP-1 $\alpha$ and VEGF) the inflammatory response to paraquat. Statistical significance was determined by one-way ANOVA followed by Student-Newman-Keuls test and cytokine levels (pg/25ug of total protein) were presented as mean \pm s.e.m (*: comparison with control mice; ${ }^{\#}$ : comparison with H5N1 infected mice; ${ }^{\circledR}$ : comparison with PQ $5 \mathrm{mg} / \mathrm{kg}$ treated mice; *, ${ }^{\#}$ and ${ }^{\circledR}: \mathrm{p}<0.05 ; * *{ }^{\# \#}$ and ${ }^{@ @}: \mathrm{P}<0.001$; error bars, s.e.m.). 
Table 4.5 Level of Cytokines, Chemokines and Growth Factors in the Cortex Following H5N1 Infection and PQ Treatment

\begin{tabular}{|c|c|c|c|c|}
\hline Cytokines & Control & H5N1 & $\mathbf{P Q}$ & H5N1 + PQ \\
\hline IL-1 $\beta$ & $16.71 \pm 1.30$ & $14.82 \pm 1.68$ & $16.12 \pm 1.71$ & $9.66 \pm 0.81 *, \#, @$ \\
\hline IL-2 & $1.44 \pm 0.36$ & $0.88 \pm 0.12$ & $1.54 \pm 0.29$ & $0.88 \pm 0.10$ \\
\hline IL-9 & $12.11 \pm 2.84$ & $2.39 \pm 0.95 *$ & $2.03 \pm 0.47 *$ & $4.72 \pm 0.95 *$ \\
\hline IL-10 & $1.98 \pm 0.86$ & $0.65 \pm 0.05$ & $1.48 \pm 0.28$ & $0.53 \pm 0.13$ \\
\hline IL-12(p70) & B.D.L & B.D.L & $0.40 \pm 0.03 * *$ & $0.51 \pm 0.02 * *$,\#\#,@@ \\
\hline Eotaxin & $2.41 \pm 0.23$ & $1.71 \pm 0.35$ & $2.20 \pm 0.30$ & $1.76 \pm 0.23$ \\
\hline GM-CSF & B.D.L & B.D.L & $0.89 \pm 0.13 * *, \#$ & $1.09 \pm 0.36 * *, \#$ \\
\hline IFN- $\gamma$ & B.D.L & B.D.L & $0.39 \pm 0.04 * *$ & $0.32 \pm 0.04 * *, \# \#, @$ \\
\hline IP-10 & $3.13 \pm 2.03$ & B.D.L & $4.86 \pm 1.80^{\#}$ & B.D.L@ \\
\hline $\mathrm{KC}$ & $2.16 \pm 0.28$ & $0.64 \pm 0.09 *$ & $2.27 \pm 0.29^{\#}$ & $1.24 \pm 0.12 *, @$ \\
\hline M-CSF & $0.75 \pm 0.13$ & $0.54 \pm 0.18$ & $0.75 \pm 0.12$ & $0.73 \pm 0.09$ \\
\hline MCP-1 & $2.33 \pm 0.91$ & B.D.L * & $1.67 \pm 0.34$ & $1.26 \pm 0.40$ \\
\hline MIP-1 $\alpha$ & B.D.L & B.D.L & $0.99 \pm 0.37 *, \#$ & $0.86 \pm 0.27 *$ *\# \\
\hline MIP-1 $\beta$ & B.D.L & B.D.L & $0.91 \pm 0.05 * *, \# \#$ & $0.73 \pm 0.10 * *, \# \#, @$ \\
\hline VEGF & $2.49 \pm 0.16$ & $4.21 \pm 1.02 *$ & $1.13 \pm 0.06$ *\#\# & $0.79 \pm 0.12 * \#$ \\
\hline
\end{tabular}




\section{Table 4.5 (continued)}

Mean fluorescent intensity of blanks, standards, and samples was measured in duplicate wells, using the Luminex 200 reader (Luminex Corp., Austin, TX). The median fluorescent intensity of blanks was subtracted from all readings and concentration of each cytokine was calculated from a 5-parameter logistic curve obtained from the known concentration of cytokines. All results were normalized with total protein concentration measured from each tissue lysate ( $\mathrm{pg} / 25 \mu \mathrm{g}$ of total protein). B.D.L represented a value below the detectable concentration range of each analyte and was regarded as zero for statistical calculation. Statistical significance was determined by one-way ANOVA followed by Student-Newman-Keuls test (*: comparison with control mice; ${ }^{*}$ : comparison with H5N1 infected mice; ${ }^{@}$ : comparison with PQ 5mg/kg treated mice; *, ${ }^{\#}$ and ${ }^{@}$ : p $<0.05$; **, ${ }^{\# \#}$ and ${ }^{@ @}$ : P $<0.001$; error bars, s.e.m.). 
In this set of experiments we examined one combination of insults, that of a neurotropic virus, H5N1 (A/VN/1203/1204) and paraquat, an agent that generates oxidative stress by a direct redox mechanism. The highly pathogenic H5N1 $(\mathrm{A} / \mathrm{VN} / 1203 / 04)$ strain is neurotropic and induces both short-term and long-term effects in the central nervous system, including a transient reduction of the dopamine (270) and a long-lasting increase in inflammation in the brain, characterized by an increase in the number of both resting and activated microglia and differential expression of a number of cytokines, chemokines and growth factors (Chapter 3).

Paraquat has been widely used throughout the world as an herbicide; compounds that have shown to have an etiological link with Parkinson's disease $(74,170)$. Paraquat is actively transported into the brain through neutral amino acid transporters (347). Once in the brain, experimental evidence has shown that paraquat acts as a direct redox cycling agent, where it first undergoes a one-step reduction catalyzed by NADPH oxidase, NOS and cellular reductases in microglial cells $(307,348)$. The paraquat cation radical then transfers its electrons to molecular oxygen that results in the formation of superoxides. Additionally, by virtue of the SNpc having significant dopamine metabolism and a significantly increased microglial density compared to other brain regions (12), there is a propensity to form hydrogen peroxide and superoxides that react with nitrated proteins to for peroxynitrites in this structure, eventually initiating apoptosis through a Bcl-2 homologous antagonist/killer (BAK) dependent mechanism (349).

Contrary to our hypothesis, the results of this study suggest that H5N1 (as a first insult) may have either no effect or may even protect the dopaminergic neurons in the substantia nigra pars compacta from paraquat-induced neurotoxicity. This conclusion is based on the findings that H5N1+PQ mice show no loss of TH-positive SNpc dopaminergic neurons and this same combination appears to suppress most of the longterm cytokine responses seen following intranasal H5N1 inoculation.

Thus, the question is raised regarding how $\mathrm{H} 5 \mathrm{~N} 1$ priming may protect the CNS (and in particular the basal ganglia) from paraquat-induced neurotoxicity and neuroinflammation? One possibility (and an area that was not examined in this thesis) is that in addition to induction of proinflammatory cytokines, H5N1 may also induce, either directly or indirectly, neurotrophic factor expression. Neurotrophins are small molecules that generally have a positive survival influence on neurons, allowing these cells to survive after a variety of insults that would normally injure or kill them (350). In the CNS, neurotrophins are expressed by both neurons and glia (astrocytes and microglia) (351) and several studies have shown that inflammation in the CNS and the subsequent release of pro-inflammatory cytokines can induce production of these proteins (351-353) (350) (351) .Thus providing a "Yin and Yang" function to microglial activation and inflammatory induction (354-357), where in the case of these two agents, there is a gradient of function towards the neurotrophins

The timing and context of the individual insults may also affect the combined "output" of these experiments. For example, Mangano et al. reported that pre-LPS infusion into the SN potentiated paraquat-induced neurotoxic effect on dopaminergic 
neuron, when PQ was delivered 2 days after the endotoxin in C57 BL/6J mice. This same combination of toxins showed opposite results when paraquat was administered 7 days after LPS infusion (358). In our experiment, the timing of the two insults could be a factor, if the 60 day interval between insults either does not allow for the reinitiation of an inflammatory response or if the initial insult is self-limiting. Examination of the cytokine profiles in the basal ganglia suggests that these possibilities exist, as there is no example of higher levels of cytokine induction in the $\mathrm{H} 5 \mathrm{~N} 1+$ paraquat condition compared to paraquat or $\mathrm{H} 5 \mathrm{~N} 1$ alone. This preconditioned repression has been described after exposure to ionizing radiation (359), ischemia (360), or LPS (358). Although the mechanism for this inflammatory repression is not fully elucidated, it has been shown that elevation of heat shock protein 70 (HSP70) (361), which ultimately inhibits expression of the nuclear factor- $\kappa \mathrm{B}(\mathrm{NF}-\kappa \mathrm{B})$ pathway, can modulate the inflammatory response. NF- $\kappa \mathrm{B}$ is an essential transcription factor that regulates transcription of genes shown to be involved in immune function, inflammation, and apoptosis (362-364). One mechanism for the protective effects of HSP70 appears to be its ability to inhibit reinduction of cytokines by blocking NF- $\kappa \mathrm{B}$ p65 nuclear translocation and $\mathrm{I} \kappa \mathrm{B} \alpha$ degradation $(361,365,366)$. Further studies are needed to determine if this mechanism is involved in the influenza induced cytokine repression.

It is also possible that the dosage of paraquat used in this study $(5 \mathrm{mg} / \mathrm{kg})$ as the second stressor may not have been at a high enough level to induce sufficient oxidative stress on dopaminergic neurons to generate a cytokine response (that would overcome any inhibition, see above). This hypothesis could be tested by examining cytokine reinduction after administration of higher levels of paraquat (such as $10 \mathrm{mg} / \mathrm{kg}$ ). However, Klinworth et al. (367) reported that paraquat was unable to induce midbrain microglia to produce any proinflammatory cytokines and suggested that any of paraquat's inflammatory effects are not a direct response from microglia but are a direct result of dopaminergic neuron damage. As the dose of paraquat we used $(5 \mathrm{mg} / \mathrm{kg})$ does not produce a massive cell loss of the dopaminergic neurons nor effect release of dopamine in the striatum, this second insult (paraquat) may either not be a sufficient stressor at these low levels or may not be a factor in any "multiple hit" cascade for Parkinson's disease. A literature search looking for direct cases of proven paraquat-induced parkinsonism in humans shows no case reports (368) and it is possible other agents that induce oxidative stress in the brain, such as MPTP or rotenone, that work via direct inhibition of Complex I in the electron transport chain $(12,369)$ and have been shown to induce human parkinsonism, will provide evidence for this "multiple hit" hypothesis.

In conclusion, we found that acute $\mathrm{H} 5 \mathrm{~N} 1$ influenza infection, although producing a long-term activation of microglia with proinflammatory cytokine production, appears to protect the dopaminergic neurons from paraquat-induced neurotoxicity. We suggest that the timing and the context of each insult may be critical for determining the consequence of combinatorial effects in the "multiple hit" hypothesis. 


\section{CHAPTER 5. IMPLICATIONS, LIMITATIONS AND FUTURE STUDIES}

Since James Parkinson first described Parkinson's disease in 1817 (1), more than 50,000 research papers have been published on the topic of Parkinson's disease (370). Despite the knowledge accumulated and progress made in understanding PD, thus far, the mechanisms underlying the development of Parkinson's disease have not been fully elucidated.

During the last two decades, studies in Parkinson's disease-related genetics have shed light on some of the pathogenesis of Parkinson's disease, as genetic mutations linked to the early onset and familial forms of PD were identified. However, the PD cases related to individual genetic mutations account for at most $10 \%$ of the total PD cases (7).

Other factors from the environment, such as infectious agents have also been suggested as a risk factor in the development of Parkinson's disease. Reports of influenza-associated neurological symptoms are found as far back as 1385 and have continued through more recent influenza outbreaks (5). Among the influenza pandemics in the twentieth century, the 1918 Spanish flu (H1N1) pandemic was the most severe, resulting in 20-100 million casualties, with an unusually high rate of mortality in the young (371). A significant number of patients exhibited unusual neurological symptoms known as Encephalitis lethargica (EL) (24). Approximately 50\% of patients surviving EL later developed post-encephalitic Parkinsonism (PEP), providing epidemiological links between EL and PEP (5, 42, 372), although direct evidence linking H1N1 infection and the subsequent development of EL or PEP has not been published.

The recently publicized highly pathogenic avian influenza (HPAI/H5N1) virus is, and continues to be, a severe threat to public health. Until May 2010, the H5N1 global outbreak has caused 498 human cases of flu and 294 deaths, resulting in a $60 \%$ mortality rate (255). Like the $1918 \mathrm{H} 1 \mathrm{~N} 1$ influenza virus, H5N1 virus originated in avian species, and demonstrates its highest mortality rate in young adults (371). In at least two of the reported human cases of $\mathrm{H} 5 \mathrm{~N} 1$, patients died with acute encephalitis without the classic respiratory symptoms, and in these patients, H5N1 influenza virus was detected in their cerebrospinal fluid (207). This suggests that H5N1 virus can infect the CNS, and even survivors may have some degree of encephalitis at some point in the course of the H5N1 infection $(269,373)$. Therefore, the primary objectives of this dissertation are A) to determine if intranasally delivered $\mathrm{H} 5 \mathrm{~N} 1$ virus can infect the CNS and induce neuroinflammation, B) to determine if the H5N1 influenza virus infection in the CNS results in parkinsonian pathology.

In many cases of encephalitis or toxin-induced parkinsonism the offending agent may cause a long lasting immune response in the brain, persisting many years after the initial insult has been resolved, leading to a "hit and run" mechanism where the original insult is no longer present but the secondary sequelae persists (5). If it is recognized that influenza activates the innate CNS immune system $(225,374)$, identification of potential second hits that may lead to development of parkinsonian symptoms is useful. 
One class of agents that could act as a "second hit" includes agricultural products, such as paraquat. Each of these chemicals induce oxidative stress and the generation of free radicals, either through blockade of complex I (rotenone) $(375,376)$ or direct free radical formation, independent of complex I inhibition (paraquat) $(347,377)$. Whatever the cause of increased oxidative stress, it is clear that prior activation of microglia increases sensitivity to these agents $(378,379)$. Therefore, the secondary objective of this dissertation is to determine if $\mathrm{H} 5 \mathrm{~N} 1$ priming of the immune system potentiates paraquatinduced neurotoxicity and neurodegeneration.

\subsection{Chapter 2}

In Chapter 2, I demonstrated that the highly infectious, neurotropic A/VN/1203/04 (H5N1) virus travels from the peripheral nervous system into the CNS, first appearing in the Solitary nucleus and adjacent dorsal motor nucleus of $\mathrm{X}$ in the brainstem. This pattern of viral spread, from the periphery to higher levels of the neuraxis, mimics the progression of Parkinson's disease described by Braak (121) . Braak postulated that some viruses might infect the mesenteric and myenteric (Auerbach's) plexi of the enteric nervous system and be retrogradely transported into the dorsal motor nucleus (DMN) of the vagus nerve, since a synuclein pathology characterized by swollen neurites and inclusions were commonly found in the fibers that project to this region from the vagus nerve (enteric origin) as well as the neurons of this nucleus (380). In addition to considering the physiological relevance of Braak's hypothesis to Parkinson's disease pathology, his insight leads me to examine whether the H5N1 virus can be transported intraneuronally. Using microfluidic chambers, I confirmed that $\mathrm{H} 5 \mathrm{~N} 1$ is transported retrogradely. I also observed the activation of microglia as well as the phosphorylation and aggregation of alpha-synuclein that persists long after the presence of viral protein, in regions infected by $\mathrm{H} 5 \mathrm{~N} 1$ virus.

\subsubsection{Implications and limitations}

In general, when performing influenza research using mammalian models, ferrets are thought to be the ideal animal model as they are susceptible to the human influenza viruses and develop upper respiratory tract infection and clinical signs such as fever, rhinitis, and sneezing, similar to humans (381). The likely reason for this similarity to humans is the presence of $\alpha 2,3$ sialic acid oligosaccharide linkages, which bind preferentially to the H5N1 influenza virus (202). The distribution of the receptors in ferret resembles that seen in humans and initially mediates lower respiratory tract infection in ferret (382). The drawbacks of the ferret model are the high cost for housing and the difficulty in obtaining influenza seronegative animals (382). For our purposes, there is little known about the ferret as a Parkinson's disease model, and mice provide a useful genetic as well as environmental model to study the etiology of PD. 
In this study, we choose not to use ferrets, but instead used C57BL/6J mice since these animals are the preferred species and strain for experimental Parkinson's disease research (12). However, what is advantageous for ferrets may be a limiting factor in mice since human influenza virus generally doesn't easily infect mice unless the virus has been "adapted" by serial passage into the lung of mice. Adaptation may arise due to mutations that may occur during this process, and as this virus mutates the original pathogenesis of the influenza virus may change (382). However, the strain of highly pathogenic H5N1 influenza virus used in this study (A/VN/1203/04), collected from a human victim, infects mice without adaptation (263) and thus any experimental results obtained are likely direct and correlatable effects to humans and will not reflect alterations due to serial passage-induced mutations.

Although we were able to infect mice without adaptation, different strains of mice have been shown to have differential susceptibility to influenza virus depending on polymorphisms of the interferon-regulated Mx gene in each strain (382). Mx protein encoded by the Mx gene inhibits influenza virus replication. Thus, mice carrying $\mathrm{Mx}+$ alleles such as $\mathrm{T} 9$ are resistant and mice carrying $\mathrm{Mx}$ - alleles are susceptible to influenza virus infection (259). C57BL/6J, a standard inbred laboratory strain, possess defective alleles of the Mx1 gene, so they don't have a complete antiviral defense system (259).

\subsubsection{Future studies}

One of the keys to understanding pathogenesis of any viral-induced infection of the CNS is to determine how the virus enters the CNS. We suggested that the sensory and autonomic nervous system, particularly the vagus nerve which innervates the lung and the peritoneal cavity, may provide "a back door" for the H5N1 virus to reach the CNS, while evading the host immune surveillance in the periphery. We showed, empirically, the in vitro intraneuronal transport of H5N1 virus using adult DRG cells grown in microfluidic chambers. We also showed using transmission electron microscopy the presence of cellular inclusions that have all the morphological characteristics of viral particles in myelinated nerves in the brainstem; and what would have to be influenza virus as the animals used are certified pathogen free and were raised in a pathogen free environment with the additional finding that control non-inoculated animals never contained these structures. However, this does not provide evidence that the vagus nerve is the single route of CNS infection of $\mathrm{H} 5 \mathrm{~N} 1$ in vivo. This could be directly tested by performing unilateral vagotomy followed by $\mathrm{H} 5 \mathrm{~N} 1$ administration. If $\mathrm{H} 5 \mathrm{~N} 1$ virus is detected only in the side with an intact vagus route at day 2-3 post-operation, the period when the $\mathrm{H} 5 \mathrm{~N} 1$ virus begins to appear in the solitary nucleus, it would prove that the vagus nerve is one route for the H5N1 virus to infect the CNS. Further studies could them be performed to examine: 1) the mechanisms in which virus enters the nerve (clathrin coated pits? (383)) and 2) how the virus is transported to the cell body (microtubule dependent transport? (384)).

It will also be critical to determine what are the genetic signatures that mark a particular influenza strain as neurotropic? This can be examined through two approaches. 
First, we can randomly generate a recombinant strain between neurovirulent A/VN/1203/04 (H5N1) and non-neurovirulent strain, such as A/Jap/305/57 (H2N2) (385). Second, we can also mutate specific gene sequences in the H5N1 genome to identify the genes related to neurotropism. These studies could be performed using the microfluidic chambers we used to test the intraneuronal transport of H5N1 virus in chapter 2 (270). We could also use a direct in vivo test where we inoculate the chimeric viruses into mice and ask if the brains get infected. This information would be beneficial for informing clinicians and other public health officials in the case of pandemic flu.

\subsection{Chapter 3}

In Chapter 3, I demonstrated that H5N1 infection can produce a pathology that has similarities with those seen in Parkinson's disease. I found a transient decrease in the number of TH-positive neurons in the SNpc as well as a similar transient decrease in the amount of striatal dopamine content. These transient decreases were seen at days post infection (dpi) 10, 21 and 60, but recovered by 90 dpi. The dopaminergic neurons of the SNpc appeared shrunken and atrophic at 10-60 dpi, but recovered by 90 dpi. Although we saw an increase in TH-positive neuron number, we saw no evidence of neurogenesis in dopaminergic neurons. One possible explanation for these observations in that active H5N1 infection causes SNpc dopamine neurons to transiently reduce their metabolic capacity compromising both $\mathrm{TH}$ activity and reducing cell size.

Unlike the transient changes in the dopaminergic system, the effects of H5N1 infection on the serotonergic system in the brain were more persistent, displaying a significant decrease of serotonin in the striatum, cortex and brainstem through $90 \mathrm{dpi}$. Due to limitations in animal protocols, these studies could not be expanded beyond 90 days, so if a later recovery of this neurotransmitter would occur, we would not have detected it.

I also found a long-term activation of microglia with cytokine production. Since activated microglia function in surveillance and response to insult, their continued presence in certain regions of the brain may make these regions particularly vulnerable to later insults or even normal age-induced changes, which could exacerbate, or cause, a degenerative response that would not normally occur.

\subsubsection{Implications and limitations}

An important question to ask is whether the effects of the H5N1 strain of influenza virus on the basal ganglia and the brain in general, are direct or indirect. We see the presence of $\mathrm{H} 5 \mathrm{~N} 1$ in the brain, however we do not know if the progression of this infection is mediated by direct viral-neuronal interactions and/or viral-microglia interactions; or if the damage is secondary to humoral-nervous system immune interactions. 
The cytokine profiles we observed support both types of effects. In support of indirect action, there is a slight delay of most of the cytokine induction in the brain compared to the point of cytokine induction in the lungs, which is the primary target of H5N1 infection. A similar pattern of delayed cytokine expression in the brain was also reported by another group, where they showed that mice had a massive and sustained infiltration of macrophages and neutrophils with a strong induction of IL-1 $\beta$, TNF- $\alpha$, IFN- $\gamma$, IL-6, MIP-1 $\alpha$, MIP-2, and RANTES in the lung at 3-5 day. Delayed induction of IL- $1 \beta$, TNF- $\alpha$, IFN- $\gamma$, IL-6, MIP-2, and MIP- $1 \alpha$ in the brain were observed at day 6 postinfection (371).

In support of viral-neuronal interactions and/or viral-microglia interactions, we saw a reinduction of cytokine expression after H5N1 virus was no longer present in the brain (21 days post infection) and no other insult was provided. Some cytokines were reinduced both in the SN and the lung. In particular, IL-13 and IL-2, that are known to be derived from microglia and lymphocytes and influence an inflammation response and neuronal survival (386), were only observed in a second response in the SN and striatum respectively. Taken together, this evidence suggests that both a direct viral-neuronal and viral-microglia interaction, and a humoral-nervous system immune interaction may occur during the course of H5N1 virus infection.

It is also important to determine what might be the stimulus that generates the reinduction of cytokines in the brain. One possibility is that there is a secondary bacterial infection that occurs due to decreased resistance generated by the initial viral infection. However, the secondary bacterial infection is less likely to occur in the BSL3+ environment, which provides an isolated and secured environment for these animals (387). Our group, as well as other independent groups, report that H5N1 virus is not detectable beyond 15 days after infection in mice, by immunohistochemical methods or PCR $(270,343,388)$. However, while not easily detectable, some of the viral components might not be cleared by the primary immune response that seems to end by day 21 , and may trigger the secondary immune response at 60 and 90 dpi. A latent H5N1 infection or secondary infection from dormant $\mathrm{H} 5 \mathrm{~N} 1$ influenza virus has not yet been described. Another possible cause for the reinduction of cytokine expression is found based on its temporal correlation of reinduction with that of dopamine. We detected increased levels of DA in both the substantia nigra (by expression of tyrosine hydroxylase and HPLC) and striatum (HPLC) at $60 \mathrm{dpi}$, which may cause oxidative stress through quinone formation and activate microglia (310, 336-339). That dopamine itself can generate a redox signal has been shown both in vitro (389) and in vivo (390-392).

\subsubsection{Future studies}

The CNS has been viewed as immune privileged site due to the presence of the blood brain barrier, the reduced major histocompatibility complex (MHC) class II expression on cells in the brain and the low T-cell and B-cell number within the CNS (393). This concept may be questioned, since the immune response in the CNS is

revealed to be tightly regulated by the cross communication of neuron and glial cells with 
immune cells (394). In line with this view, it is not surprising that peripheral leukocytes, including CD8+ and CD4+ T cells and granulocytes infiltrate the CNS and modulate the immune response, controlling disease progression in many chronic neurodegenerative disorders, including multiple sclerosis (MS) and PD $(301,394)$.

Although subsequent induction of cytokine expression and increased numbers of activated microglia appeared 60 days post-infection, activated microglia may not be the only initiator of latent cytokine induction. We have hypothesized that T-cells may also play an important role in both the acute and chronic immune responses in our model. We are interested in knowing if the interaction between lymphocytes and microglia is essential for microglia activation and the induction of proinflammatory cytokines. One way to test this hypothesis is through the use of transgenic mice, such as the RAG-1 (recombination activating gene-1) deficient mice that lack T-cells (395), LAG-3 null mice that lack natural killer cells (396) and MT mice (B6-Igh6-6tm1Cgn) that lack Bcells (397). One caveat in these experiments is that since these mice don't have a complete immune system; they may show different susceptibility and pathophysiology in response to acute $\mathrm{H} 5 \mathrm{~N} 1$ viral infection. However, testing these models with the proper controls may suggest which population of peripheral immune cells is critical for the communication with neurons and glia in the CNS.

\subsection{Chapter 4}

In Chapter 4, we tested if acute $\mathrm{H} 5 \mathrm{~N} 1$ priming affects paraquat-induced neurotoxicity. We detected a transient reduction of the dopamine output in striatum, a long-lasting increase in proinflammatory cytokines and chemokines, and an increase in the number of both resting and activated microglia in the $\mathrm{SN}$ at day 60 post infection. These observations lead us to hypothesize that the change in the microenvironment of the CNS produced by $\mathrm{H} 5 \mathrm{~N} 1$ infection could make the basal ganglia sensitive to PQ-induced oxidative stress.

In contrast to our expectations, the results suggest that $\mathrm{H} 5 \mathrm{~N} 1$ priming might protect the dopaminergic neurons from PQ-induced neurotoxicity and desensitize the immune response produced by $\mathrm{PQ}$ in the lung, $\mathrm{SN}$, and striatum. The desensitization effect of $\mathrm{H} 5 \mathrm{~N} 1$ priming was the most obvious in the $\mathrm{SN}$.

\subsubsection{Implications and limitations}

Many cytokines, including IL-1 $\alpha$, IL-1 $\beta$, IL-6, IL-10, IL-13, and TNF- $\alpha$ have pleomorphic functions and regulate the activation, proliferation and suppression of cells with different time course and ability (398). Each cell is also a part of a huge cellular network which is composed of thousands of communicating cells. Therefore, complexity in the net effect from the milieu of cytokines is inevitable. 
One cell type we did not examine in context of influenza (and paraquat) effects was astrocytes; and any pathology seen (or not seen) needs to take the biology of these cells into the calculation of net effects. Several studies showed that astrocytes produce neurotropic factors, including nerve growth factor (NGF) and glial cell line-derived neurotrophic factor (GDNF) that respond to inflammatory stimulation including lipopolysaccharide and pro-inflammatory cytokines such as TNF- $\alpha$, interleukin- $1 \beta$ (IL$1 \beta)$ and interferon- $\gamma($ IFN- $\gamma),(351-353)$ (350) (351).

The immune response is a very dynamic process. Different cells may be involved in the immune response at different times during the course of infection. For example, after exposure to MPTP, microglia in the SN are quickly activated and proliferate in 1-2 days, T-cells then begin to infiltrate at day 2-7, and later, astrocytes become activated (301). Therefore, the timing and the context of the insults could significantly affect the final pathology. To further elucidate the course of events during infection and subsequent cellular effects, a non-invasive and continuous observation method would be ideal to study the effect of multiple insults as a PD model.

\subsubsection{Future studies}

$\mathrm{H} 5 \mathrm{~N} 1$ priming was protective for paraquat-induced neurotoxicity in our experimental protocol. However, we do not know at this time if the H5N1 priming is sensitizing or desensitizing mice to secondary insults, including aging, genetic mutations, other types of neurotoxin such as rotenone, and/or infectious pathogens, encountered by the subject at various time points.

Another experimental design would be to switch the sequence of insults, for example, paraquat administration followed by H5N1 infection, to determine how neurons damaged by oxidative stress respond to H5N1-induced neuroinflammation. This study has epidemiological and physiological relevance for testing the "multiple hit" hypothesis for PD. Rotenone, a mitochondrial complex 1 inhibitor and insecticide, was isolated from the roots and stems of several plants (399). This chemical has been used in one form or another as a crop insecticide since 1848 and has been recognized as a registered pesticide in the United States under the Federal Insecticide Fungicide Rodenticide Act (FIFRA) since 1947 (5). Paraquat has also been recognized as an herbicide since 1954, but it had been synthesized by the reaction of 4,4'-bypyridium with methyl iodide and used as an oxidation reduction indicator prior to used as an herbicide (400). Therefore, it is possible that people may have been exposed to paraquat or rotenone in advance of the $1918 \mathrm{flu}$ pandemic.

A $5 \mathrm{mg} / \mathrm{kg}$ dose of paraquat is approximately 40 times lower than the $50 \%$ lethal dose (196mg/kg) (401) and does not cause systemic toxicity in peripheral organs in most experimental cases in mice (402). Multiple low doses of PQ $(10 \mathrm{mg} / \mathrm{kg}$, one injection per week for 3 weeks, ip), however, produce the intraneuronal deposition of $\alpha$-synuclein and the selective loss of dopaminergic neurons and activation of microglia in C57BL/6J mice (378). In this injection paradigm, the first paraquat administration did not cause 
dopaminergic neuron loss, however it produced a substantial activation of microglia by 7 days post administration. The second paraquat injection, which was delivered a week after the first injection caused a significant loss of dopaminergic neurons. When the antiinflammatory drug, minocycline, was delivered to block microglia response after the first paraquat injection, no dopaminergic cell loss was observed (378). This observation suggests that the first PQ injection may prime the microglia and cause reversible injury to dopaminergic neurons, while the second PQ injection would trigger events causing irreversible damage to dopaminergic neurons (368).

In previous experiments, the primary insult was an acute $\mathrm{H} 5 \mathrm{~N} 1$ infection that produced a massive proinflammatory cytokine induction in both the periphery and the CNS, that may lead to reversible damage to dopaminergic neurons and the persistent activation of microglia. The secondary insult was PQ, which may influence cells in the $\mathrm{SN}$ and the CNS specifically. In view of a multiple hit hypothesis, this experimental regime can be considered as the combination of a broad systemic insult, followed by a CNS specific insult. Therefore, PQ priming followed by H5N1 infection would represent a reverse scenario.

\subsection{Conclusions}

Chapter 2 and 3 provide evidence that $\mathrm{H} 5 \mathrm{~N} 1$ induced a parkinsonian pathology. Chapter 2 and 3 demonstrated that the highly infectious neurotropic A/VN/1203/04 (H5N1) virus progresses from the peripheral nervous system into the CNS and induces three cardinal PD related pathology, A) a temporal reduction of the dopamine in striatum, B) increased phosphorylation and aggregation of alpha synuclein, C) a long-lasting, increase in inflammation within the brain, characterized by an increase in the number of both resting and activated microglia and differential expression of a number of cytokines and chemokines.

Chapter 4 demonstrates that acute $\mathrm{H} 5 \mathrm{~N} 1$ influenza infection, although it produced a long-term activation of microglia with proinflammatory cytokine production, appears to protect the dopaminergic neurons from paraquat-induced neurotoxicity.

Thus, we suggest that $\mathrm{H} 5 \mathrm{~N} 1$ virus or any pandemic influenza that activates the immune system in brain may be an important etiological agent in itself to initiate the sequelae of neurodegenerative diseases including Parkinson's disease. We also suggest that the consequence of pre-existing inflammatory response produced by $\mathrm{H} 5 \mathrm{~N} 1$ virus, however, may vary depending on the timing and the context of a subsequent insult. 


\section{LIST OF REFERENCES}

1. Parkinson J. An essay on shaking palsy. London: Sherwood, Neeley and Jones; 1817.

2. Van Den Eeden SK, Tanner CM, Bernstein AL, Fross RD, Leimpeter A, Bloch DA, et al. Incidence of Parkinson's disease: variation by age, gender, and race/ethnicity. Am J Epidemiol. 2003 Jun;157(11):1015-22.

3. Wolters E, Van Laar, Teus, Berendse, Henk. Parkinsonism \& related disorders. Amsterdam: VU University Press; 2007.

4. CR G. Basal Ganglia. Amsterdam: Elsevier; 2004.

5. Jang H, Boltz DA, Webster RG, Smeyne RJ. Viral parkinsonism. Biochim Biophys Acta. 2009 Aug;1792(7):714-21

6. Albin RL, Young AB, Penney JB. The functional anatomy of basal ganglia disorders. Trends Neurosci. 1989 Oct;12(10):366-75.

7. Farrer MJ. Genetics of Parkinson's disease: paradigm shifts and future prospects. Nat Rev Genet. 2006 Apr;7(4):306-18.

8. Klein C, Schlossmacher MG. Parkinson's disease, 10 years after its genetic revolution: multiple clues to a complex disorder. Neurology. 2007 Nov;69(22):2093-104.

9. Belin AC, Westerlund M. Parkinson's disease: A genetic perspective. Febs J. 2008 Apr;275(7):1377-83.

10. Arai H, Furuya T, Mizuno Y, Mochizuki H. Inflammation and infection in Parkinson's disease. Histol Histopathol. 2006 Jun;21(6):673-8.

11. Mena MA, de Yebenes JG. Drug-induced parkinsonism. Expert Opin Drug Saf. 2006 Nov;5(6):759-71.

12. Smeyne RJ, Jackson-Lewis V. The MPTP model of Parkinson's disease. Brain Res Mol Brain Res. 2005 Mar;134(1):57-66.

13. Sibon I, Tison F. Vascular parkinsonism. Curr Opin Neurol. 2004 Feb;17(1):4954.

14. Bower JH, Maraganore DM, Peterson BJ, McDonnell SK, Ahlskog JE, Rocca WA. Head trauma preceding PD: a case-control study. Neurology. 2003 May;60(10):1610-5.

15. Bostantjopoulou S, Katsarou Z, Petridis A. Relapsing hemiparkinsonism due to recurrent meningioma. Parkinsonism Relat Disord. 2007 Aug;13(6):372-4.

16. Chang DC, Lin JJ, Lin JC. Parkinsonism as an initial manifestation of brain tumor. Zhonghua Yi Xue Za Zhi (Taipei). 2000 Aug;63(8):658-62.

17. Langston JW, Langston EB, Irwin I. MPTP-induced parkinsonism in human and non-human primates--clinical and experimental aspects. Acta Neurol Scand Suppl. 1984 Jan;100:49-54.

18. Schiesling C, Kieper N, Seidel K, Kruger R. Review: Familial Parkinson's disease--genetics, clinical phenotype and neuropathology in relation to the common sporadic form of the disease. Neuropathol Appl Neurobiol. 2008 Jun;34(3):255-71. 
19. Ransom BR, Kunis DM, Irwin I, Langston JW. Astrocytes convert the parkinsonism inducing neurotoxin, MPTP, to its active metabolite, MPP+. Neurosci Lett. 1987 Apr;75(3):323-8.

20. Dinis-Oliveira RJ, Remiao F, Carmo H, Duarte JA, Navarro AS, Bastos ML, et al. Paraquat exposure as an etiological factor of Parkinson's disease.

Neurotoxicology. 2006 Dec;27(6):1110-22.

21. Dauer W, Przedborski S. Parkinson's disease: mechanisms and models. Neuron. 2003 Sep;39(6):889-909.

22. Bromilow RH. Paraquat and sustainable agriculture. Pest Manag Sci. 2004 Apr;60(4):340-9.

23. Tanner CM. Occupational and environmental causes of parkinsonism. Occup Med. 1992 Jul;7(3):503-13.

24. Von Economo K. Encepahlitis lethargica. Wiener Klinische Wochenschrift. 1917 May;30:581-5.

25. Goldsmith JR, Herishanu YO, Podgaietski M, Kordysh E. Dynamics of parkinsonism-Parkinson's disease in residents of adjacent kibbutzim in Israel's Negev. Environ Res. 1997 Oct;73(1-2):156-61.

26. Kumar A, Calne SM, Schulzer M, Mak E, Wszolek Z, Van Netten C, et al. Clustering of Parkinson's disease: shared cause or coincidence? Arch Neurol. 2004 Jul;61(7):1057-60.

27. Hemling N, Roytta M, Rinne J, Pollanen P, Broberg E, Tapio V, et al. Herpesviruses in brains in Alzheimer's and Parkinson's diseases. Ann Neurol. 2003 Aug;54(2):267-71.

28. Marttila RJ, Rinne UK, Halonen P, Madden DL, Sever JL. Herpesviruses and parkinsonism. Herpes simplex virus types 1 and 2, and cytomegalovirus antibodies in serum and CSF. Arch Neurol. 1981 Jan;38(1):19-21.

29. Irkec $\mathrm{C}$. The role of viral antibodies in the pathogenesis of degenerative and demyelinating diseases. Mikrobiyol Bul. 1989 Jan;23(1):40-50.

30. Elizan TS, Madden DL, Noble GR, Herrmann KL, Gardner J, Schwartz J, et al. Viral antibodies in serum and CSF of Parkinsonian patients and controls. Arch Neurol. 1979 Sep;36(9):529-34.

31. Woulfe J, Hoogendoorn H, Tarnopolsky M, Munoz DG. Monoclonal antibodies against Epstein-Barr virus cross-react with alpha-synuclein in human brain. Neurology. 2000 Nov;55(9):1398-401.

32. Bastian FO, Rabson AS, Yee CL, Tralka TS. Herpesvirus hominis: isolation from human trigeminal ganglion. Science. 1972 Oct;178(58):306-7.

33. Tomonaga K, Kobayashi T, Ikuta K. The neuropathogenesis of Borna disease virus infection. Nippon Rinsho. 2001 Aug;59(8):1605-13.

34. Iwahashi J, Tsuji K, Ishibashi T, Kajiwara J, Imamura Y, Mori R, et al. Isolation of amantadine-resistant influenza a viruses (H3N2) from patients following administration of amantadine in Japan. J Clin Microbiol. 2001 Apr;39(4):1652-3.

35. Gamboa ET, Wolf A, Yahr MD, Barden H, Hsu CC, Duffy PE, et al. Influenza A virus as a possible cause of postencephalitic Parkinsonism. Trans Am Neurol Assoc. 1973 Jan;98:177-80. 
36. Gamboa ET, Wolf A, Yahr MD, Harter DH, Duffy PE, Barden H, et al. Influenza virus antigen in postencephalitic parkinsonism brain. Detection by immunofluorescence. Arch Neurol. 1974 Oct;31(4):228-32.

37. Isgreen WP, Chutorian AM, Fahn S. Sequential parkinsonism and chorea following "mild" influenza. Trans Am Neurol Assoc. 1976 Jan;101:56-60.

38. Maurizi CP. Was a neurovirulent influenza virus the cause of amyotrophic lateral sclerosis and parkinsonism-dementia on Guam? Med Hypotheses. 1987 Jul;23(3):325-6.

39. Ravenholt RT, Foege WH. 1918 influenza, encephalitis lethargica, parkinsonism. Lancet. 1982 Oct;2(8303):860-4.

40. Moore G. Influenza and Parkinson's disease. Public Health Rep. 1977 Jan;92(1):79-80.

41. Takahashi M, Yamada T. Viral etiology for Parkinson's disease--a possible role of influenza A virus infection. Jpn J Infect Dis. 1999 Jun;52(3):89-98.

42. Casals J, Elizan TS, Yahr MD. Postencephalitic parkinsonism-a review. J Neural Transm. 1998 Oct;105(6-7):645-76.

43. Takahashi M, Yamada T. A possible role of influenza A virus infection for Parkinson's disease. Adv Neurol. 2001 Jan;86:91-104.

44. Ball MJ. Unexplained sudden amnesia, postencephalitic Parkinson's disease, subacute sclerosing panencephalitis, and Alzheimer disease: does viral synergy produce neurofibrillary tangles? Arch Neurol. 2003 Apr;60(4):641-2.

45. Sasco AJ, Paffenbarger RS, Jr. Measles infection and Parkinson's disease. Am J Epidemiol. 1985 Dec;122(6):1017-31.

46. Peatfield RC. Basal ganglia damage and subcortical dementia after possible insidious Coxsackie virus encephalitis. Acta Neurol Scand. 1987 Nov;76(5):340-5.

47. Kamei S, Hersch SM, Kurata T, Takei Y. Coxsackie B antigen in the central nervous system of a patient with fatal acute encephalitis: immunohistochemical studies of formalin-fixed paraffin-embedded tissue. Acta Neuropathol. 1990 Jan;80(2):216-21.

48. Poser CM, Huntley CJ, Poland JD. Para-encephalitic parkinsonism. Report of an acute case due to coxsackie virus type B 2 and re-examination of the etiologic concepts of postencephalitic parkinsonism. Acta Neurol Scand. 1969 Jan;45(2):199-215.

49. Walters J. Postencephalic parkinson syndrome after meningoencephalitis due to coxsackie virus group-B, type-2. New England Journal of Medicine. 1960 Jan;263:744.

50. Horta-Barbosa L, Fuccillo DA, Sever JL. Chronic viral infections of the central nervous system. Jama. 1971 Nov;218(8):1185-8.

51. Nielsen NM, Rostgaard K, Hjalgrim H, Aaby P, Askgaard D. Poliomyelitis and Parkinson's disease. Jama. 2002 Apr;287(13):1650-1.

52. Hersh BP, Rajendran PR, Battinelli D. Parkinsonism as the presenting manifestation of HIV infection: improvement on HAART. Neurology. 2001 Jan;56(2):278-9.

53. Maggi P, de Mari M, Moramarco A, Fiorentino G, Lamberti P, Angarano G. Parkinsonism in a patient with AIDS and cerebral opportunistic granulomatous lesions. Neurol Sci. 2000 Jun;21(3):173-6. 
54. Mirsattari SM, Power C, Nath A. Parkinsonism with HIV infection. Mov Disord. 1998 Jul;13(4):684-9.

55. de la Fuente-Aguado J, Bordon J, Moreno JA, Sopena B, Rodriguez A, MartinezVazquez C. Parkinsonism in an HIV-infected patient with hypodense cerebral lesion. Tuber Lung Dis. 1996 Apr;77(2):191-2.

56. Wang GJ, Chang L, Volkow ND, Telang F, Logan J, Ernst T, et al. Decreased brain dopaminergic transporters in HIV-associated dementia patients. Brain. 2004 Nov;127(Pt 11):2452-8.

57. Koutsilieri E, Sopper S, Scheller C, ter Meulen V, Riederer P. Parkinsonism in HIV dementia. J Neural Transm. 2002 May;109(5-6):767-75.

58. Robinson RL, Shahida S, Madan N, Rao S, Khardori N. Transient parkinsonism in West Nile virus encephalitis. Am J Med. 2003 Aug;115(3):252-3.

59. Minami M, Hamaue N, Hirafuji M, Saito H, Hiroshige T, Ogata A, et al. Isatin, an endogenous MAO inhibitor, and a rat model of Parkinson's disease induced by the Japanese encephalitis virus. J Neural Transm Suppl. 2006 Jan(71):87-95.

60. Misra UK, Kalita J, Pandey S, Khanna VK, Babu GN. Cerebrospinal fluid catecholamine levels in Japanese encephalitis patients with movement disorders. Neurochem Res. 2005 Sep;30(9):1075-8.

61. Nishimura M, Mizuta I, Mizuta E, Yamasaki S, Ohta M, Kaji R, et al. Tumor necrosis factor gene polymorphisms in patients with sporadic Parkinson's disease. Neurosci Lett. 2001 Sep;311(1):1-4.

62. Ogata A, Tashiro K, Pradhan S. Parkinsonism due to predominant involvement of substantia nigra in Japanese encephalitis. Neurology. 2000 Aug;55(4):602.

63. Haraguchi T, Ishizu H, Terada S, Takehisa Y, Tanabe Y, Nishinaka T, et al. An autopsy case of postencephalitic parkinsonism of von Economo type: some new observations concerning neurofibrillary tangles and astrocytic tangles. Neuropathology. 2000 Jun;20(2):143-8.

64. Ogata A, Tashiro K, Nukuzuma S, Nagashima K, Hall WW. A rat model of Parkinson's disease induced by Japanese encephalitis virus. J Neurovirol. 1997 Apr;3(2):141-7.

65. Shoji H, Watanabe M, Itoh S, Kuwahara H, Hattori F. Japanese encephalitis and parkinsonism. J Neurol. 1993 Jan;240(1):59-60.

66. Miyasaki K, Takayoshi F. Parkinsonism following encephalitis of unknown etiology. J Neuropathol Exp Neurol. 1977 Jan;36(1):1-8.

67. Cerna F, Mehrad B, Luby JP, Burns D, Fleckenstein JL. St. Louis encephalitis and the substantia nigra: MR imaging evaluation. AJNR Am J Neuroradiol. 1999 Aug;20(7):1281-3.

68. Pranzatelli MR, Mott SH, Pavlakis SG, Conry JA, Tate ED. Clinical spectrum of secondary parkinsonism in childhood: a reversible disorder. Pediatr Neurol. 1994 Mar;10(2):131-40.

69. Reyes MG, Gardner JJ, Poland JD, Monath TP. St Louis encephalitis. Quantitative histologic and immunofluorescent studies. Arch Neurol. 1981 Jun;38(6):329-34.

70. Carvey PM, Punati A, Newman MB. Progressive dopamine neuron loss in Parkinson's disease: the multiple hit hypothesis. Cell Transplant. 2006 Oct;15(3):239-50. 
71. Cory-Slechta DA, Thiruchelvam M, Barlow BK, Richfield EK. Developmental pesticide models of the Parkinson's disease phenotype. Environ Health Perspect. 2005 Sep;113(9):1263-70.

72. Sulzer D. Multiple hit hypotheses for dopamine neuron loss in Parkinson's disease. Trends Neurosci. 2007 May;30(5):244-50.

73. Williams AC, Ramsden DB. Autotoxicity, methylation and a road to the prevention of Parkinson's disease. J Clin Neurosci. 2005 Jan;12(1):6-11.

74. McCormack AL, Thiruchelvam M, Manning-Bog AB, Thiffault C, Langston JW, Cory-Slechta DA, et al. Environmental risk factors and Parkinson's disease: selective degeneration of nigral dopaminergic neurons caused by the herbicide paraquat. Neurobiol Dis. 2002 Jul;10(2):119-27.

75. Nerlich AG, Nerlich ML, Langer I, Demling RH. Release of amino-terminal procollagen peptides in paraquat-induced acute pulmonary fibrosis. Exp Mol Pathol. 1984 Jun;40(3):311-9.

76. Bertrand E, Lechowicz W, Szpak GM, Dymecki J. Qualitative and quantitative analysis of locus coeruleus neurons in Parkinson's disease. Folia Neuropathol. 1997 Jan;35(2):80-6.

77. Gai WP, Blumbergs PC, Geffen LB, Blessing WW. Age-related loss of dorsal vagal neurons in Parkinson's disease. Neurology. 1992 Nov;42(11):2106-11.

78. Wakabayashi K, Takahashi H. The intermediolateral nucleus and Clarke's column in Parkinson's disease. Acta Neuropathol. 1997 Sep;94(3):287-9.

79. Lotharius J, Brundin P. Pathogenesis of Parkinson's disease: dopamine, vesicles and alpha-synuclein. Nat Rev Neurosci. 2002 Dec;3(12):932-42.

80. German DC, Manaye K, Smith WK, Woodward DJ, Saper CB. Midbrain dopaminergic cell loss in Parkinson's disease: computer visualization. Annals of Neurology. 1989 Jan;26(4):507-14.

81. Damier P, Hirsch EC, Agid Y, Graybiel AM. The substantia nigra of the human brain. II. Patterns of loss of dopamine-containing neurons in Parkinson's disease. Brain. 1999 Jan;122(8):1437-48.

82. Nurmi E, Ruottinen HM, Bergman J, Haaparanta M, Solin O, Sonninen P, et al. Rate of progression in Parkinson's disease: a 6-[18F]fluoro-L-dopa PET study. Mov Disord. 2001 May;16(4):608-15.

83. Forno LS. Neuropathology of Parkinson's disease. J Neuropathol Exp Neurol. 1996 Mar;55(3):259-72.

84. Lewy F. Zur pathologischen Anatomie der Paralysis agitans. Deutsche Zeitschrift fur Nervenheilkunde. 1913 Jan;50:50-5.

85. Kidd M. Paired helical filaments in electron microscopy of Alzheimer's disease. Nature. 1963 Jan;197:192-3.

86. Tompkins MM, Hill WD. Contribution of somal Lewy bodies to neuronal death. Brain Res. 1997 Nov;775(1-2):24-9.

87. Ross CA, Poirier MA. Opinion: What is the role of protein aggregation in neurodegeneration? Nat Rev Mol Cell Biol. 2005 Nov;6(11):891-8.

88. Johnston JA, Ward CL, Kopito RR. Aggresomes: a cellular response to misfolded proteins. J Cell Biol. 1998 Dec;143(7):1883-98. 
89. Taylor JP, Tanaka F, Robitschek J, Sandoval CM, Taye A, Markovic-Plese S, et al. Aggresomes protect cells by enhancing the degradation of toxic polyglutamine-containing protein. Hum Mol Genet. 2003 Apr;12(7):749-57.

90. McNaught KS, Shashidharan P, Perl DP, Jenner P, Olanow CW. Aggresomerelated biogenesis of Lewy bodies. Eur J Neurosci. 2002 Dec;16(11):2136-48.

91. Auluck PK, Chan HY, Trojanowski JQ, Lee VM, Bonini NM. Chaperone suppression of alpha-synuclein toxicity in a Drosophila model for Parkinson's disease. Science. 2002 Feb;295(5556):865-8.

92. Schlossmacher MG, Frosch MP, Gai WP, Medina M, Sharma N, Forno L, et al. Parkin localizes to the Lewy bodies of Parkinson's disease and dementia with Lewy bodies. Am J Pathol. 2002 May;160(5):1655-67.

93. Ardley HC, Scott GB, Rose SA, Tan NG, Markham AF, Robinson PA. Inhibition of proteasomal activity causes inclusion formation in neuronal and non-neuronal cells overexpressing Parkin. Mol Biol Cell. 2003 Nov;14(11):4541-56.

94. Spillantini MG, Schmidt ML, Lee VM, Trojanowski JQ, Jakes R, Goedert M. Alpha-synuclein in Lewy bodies. Nature. 1997 Aug;388(6645):839-40.

95. Polymeropoulos MH, Lavedan C, Leroy E, Ide SE, Dehejia A, Dutra A, et al. Mutation in the alpha-synuclein gene identified in families with Parkinson's disease. Science. 1997 Jun;276(5321):2045-7.

96. Eliezer D, Kutluay E, Bussell R, Jr., Browne G. Conformational properties of alpha-synuclein in its free and lipid-associated states. J Mol Biol. 2001 Apr;307(4):1061-73.

97. Jensen PH, Hojrup P, Hager H, Nielsen MS, Jacobsen L, Olesen OF, et al. Binding of Abeta to alpha- and beta-synucleins: identification of segments in alpha-synuclein/NAC precursor that bind Abeta and NAC. Biochem J. 1997 Apr;323:539-46.

98. Park SM, Jung HY, Kim TD, Park JH, Yang CH, Kim J. Distinct roles of the Nterminal-binding domain and the C-terminal-solubilizing domain of alphasynuclein, a molecular chaperone. J Biol Chem. 2002 Aug;277(32):28512-20.

99. Kim TD, Paik SR, Yang CH. Structural and functional implications of C-terminal regions of alpha-synuclein. Biochemistry. 2002 Nov;41(46):13782-90.

100. Engelender S, Kaminsky Z, Guo X, Sharp AH, Amaravi RK, Kleiderlein JJ, et al. Synphilin-1 associates with alpha-synuclein and promotes the formation of cytosolic inclusions. Nat Genet. 1999 May;22(1):110-4.

101. Wakabayashi K, Engelender S, Yoshimoto M, Tsuji S, Ross CA, Takahashi H. Synphilin-1 is present in Lewy bodies in Parkinson's disease. Ann Neurol. 2000 Apr;47(4):521-3.

102. Abeliovich A, Schmitz Y, Farinas I, Choi-Lundberg D, Ho WH, Castillo PE, et al. Mice lacking alpha-synuclein display functional deficits in the nigrostriatal dopamine system. Neuron. 2000 Jan;25(1):239-52.

103. Chen PE, Specht CG, Morris RG, Schoepfer R. Spatial learning is unimpaired in mice containing a deletion of the alpha-synuclein locus. Eur J Neurosci. 2002 Jul;16(1):154-8.

104. Maroteaux L, Campanelli JT, Scheller RH. Synuclein: a neuron-specific protein localized to the nucleus and presynaptic nerve terminal. J Neurosci. 1988 Aug;8(8):2804-15. 
105. Okochi M, Walter J, Koyama A, Nakajo S, Baba M, Iwatsubo T, et al.

Constitutive phosphorylation of the Parkinson's disease associated alphasynuclein. J Biol Chem. 2000 Jan;275(1):390-7.

106. Anderson JP, Walker DE, Goldstein JM, de Laat R, Banducci K, Caccavello RJ, et al. Phosphorylation of Ser-129 is the dominant pathological modification of alpha-synuclein in familial and sporadic Lewy body disease. J Biol Chem. 2006 Oct;281(40):29739-52.

107. Jellinger KA. Lewy body-related alpha-synucleinopathy in the aged human brain. J Neural Transm. 2004 Oct;111(10-11):1219-35.

108. von Bohlen Und Halbach O. Synucleins and their relationship to Parkinson's disease. Cell Tissue Res. 2004 Oct;318(1):163-74.

109. Li J, Uversky VN, Fink AL. Conformational behavior of human alpha-synuclein is modulated by familial Parkinson's disease point mutations A30P and A53T. Neurotoxicology. 2002 Oct;23(4-5):553-67.

110. Conway KA, Harper JD, Lansbury PT. Accelerated in vitro fibril formation by a mutant alpha-synuclein linked to early-onset Parkinson's disease. Nat Med. 1998 Nov;4(11):1318-20.

111. Narhi L, Wood SJ, Steavenson S, Jiang Y, Wu GM, Anafi D, et al. Both familial Parkinson's disease mutations accelerate alpha-synuclein aggregation. J Biol Chem. 1999 Apr;274(14):9843-6.

112. Uversky VN, Li J, Fink AL. Pesticides directly accelerate the rate of alphasynuclein fibril formation: a possible factor in Parkinson's disease. FEBS Lett. $2001 \mathrm{Jul} ; 500(3): 105-8$.

113. Bennett MC, Bishop JF, Leng Y, Chock PB, Chase TN, Mouradian MM. Degradation of alpha-synuclein by proteasome. J Biol Chem. 1999 Nov;274(48):33855-8.

114. Rideout HJ, Larsen KE, Sulzer D, Stefanis L. Proteasomal inhibition leads to formation of ubiquitin/alpha-synuclein-immunoreactive inclusions in PC12 cells. J Neurochem. 2001 Aug;78(4):899-908.

115. Shimura H, Schlossmacher MG, Hattori N, Frosch MP, Trockenbacher A, Schneider R, et al. Ubiquitination of a new form of alpha-synuclein by parkin from human brain: implications for Parkinson's disease. Science. 2001 Jul;293(5528):263-9.

116. Leroy E, Boyer R, Auburger G, Leube B, Ulm G, Mezey E, et al. The ubiquitin pathway in Parkinson's disease. Nature. 1998 Oct;395(6701):451-2.

117. Cuervo AM, Stefanis L, Fredenburg R, Lansbury PT, Sulzer D. Impaired degradation of mutant alpha-synuclein by chaperone-mediated autophagy. Science. 2004 Aug;305(5688):1292-5.

118. Pan T, Kondo S, Le W, Jankovic J. The role of autophagy-lysosome pathway in neurodegeneration associated with Parkinson's disease. Brain. 2008 Aug;131(8):1969-78.

119. Braak H, Braak E, Yilmazer D, de Vos RA, Jansen EN, Bohl J. Pattern of brain destruction in Parkinson's and Alzheimer's diseases. J Neural Transm. 1996 Jan;103(4):455-90. 
120. Braak H, Ghebremedhin E, Rub U, Bratzke H, Del Tredici K. Stages in the development of Parkinson's disease-related pathology. Cell Tissue Res. 2004 Oct;318(1):121-34.

121. Braak H, Del Tredici K, Rub U, de Vos RA, Jansen Steur EN, Braak E. Staging of brain pathology related to sporadic Parkinson's disease. Neurobiol Aging. 2003 Mar;24(2):197-211.

122. Abbott RD, Ross GW, Petrovitch H, Tanner CM, Davis DG, Masaki KH, et al. Bowel movement frequency in late-life and incidental Lewy bodies. Mov Disord. 2007 Aug;22(11):1581-6.

123. Stiasny-Kolster K, Doerr Y, Moller JC, Hoffken H, Behr TM, Oertel WH, et al. Combination of 'idiopathic' REM sleep behaviour disorder and olfactory dysfunction as possible indicator for alpha-synucleinopathy demonstrated by dopamine transporter FP-CIT-SPECT. Brain. 2005 Jan;128(Pt 1):126-37.

124. Hawkes CH, Del Tredici K, Braak H. Parkinson's disease: a dual-hit hypothesis. Neuropathol Appl Neurobiol. 2007 Dec;33(6):599-614.

125. McGeer PL, McGeer EG. Glial cell reactions in neurodegenerative diseases: pathophysiology and therapeutic interventions. Alzheimer Dis Assoc Disord. 1998 Jan;12 Suppl 2:S1-6.

126. Hirsch EC, Breidert T, Rousselet E, Hunot S, Hartmann A, Michel PP. The role of glial reaction and inflammation in Parkinson's disease. Ann N Y Acad Sci. 2003 Jun;991:214-28.

127. Nagatsu T, Mogi M, Ichinose H, Togari A. Cytokines in Parkinson's disease. J Neural Transm Suppl. 2000 Jan(58):143-51.

128. Dobbs RJ, Charlett A, Purkiss AG, Dobbs SM, Weller C, Peterson DW. Association of circulating TNF-alpha and IL-6 with ageing and parkinsonism. Acta Neurol Scand. 1999 Jul;100(1):34-41.

129. Mogi M, Harada M, Kondo T, Riederer P, Inagaki H, Minami M, et al. Interleukin-1 beta, interleukin-6, epidermal growth factor and transforming growth factor-alpha are elevated in the brain from parkinsonian patients. Neurosci Lett. 1994 Oct;180(2):147-50.

130. Kaltschmidt B, Baeuerle PA, Kaltschmidt C. Potential involvement of the transcription factor NF-kappa B in neurological disorders. Mol Aspects Med. 1993 Jan;14(3):171-90.

131. Langston JW, Forno LS, Tetrud J, Reeves AG, Kaplan JA, Karluk D. Evidence of active nerve cell degeneration in the substantia nigra of humans years after 1methyl-4-phenyl-1,2,3,6-tetrahydropyridine exposure. Ann Neurol. 1999 Oct;46(4):598-605.

132. Smith PF. Inflammation in Parkinson's disease: an update. Curr Opin Investig Drugs. 2008 May;9(5):478-84.

133. Hernan MA, Logroscino G, Garcia Rodriguez LA. Nonsteroidal antiinflammatory drugs and the incidence of Parkinson's disease. Neurology. 2006 Apr;66(7):1097-9.

134. Chen H, Jacobs E, Schwarzschild MA, McCullough ML, Calle EE, Thun MJ, et al. Nonsteroidal antiinflammatory drug use and the risk for Parkinson's disease. Ann Neurol. 2005 Dec;58(6):963-7. 
135. Cotzias GC, Van Woert MH, Schiffer LM. Aromatic amino acids and modification of parkinsonism. N Engl J Med. 1967 Feb;276(7):374-9.

136. Hauser RA. Levodopa: past, present, and future. Eur Neurol. 2009 Jan;62(1):1-8.

137. Quinones H, Collazo R, Moe OW. The dopamine precursor Ldihydroxyphenylalanine is transported by the amino acid transporters rBAT and LAT2 in renal cortex. Am J Physiol Renal Physiol. 2004 Jul;287(1):F74-80.

138. Nutt JG, Woodward WR, Anderson JL. The effect of carbidopa on the pharmacokinetics of intravenously administered levodopa: the mechanism of action in the treatment of parkinsonism. Ann Neurol. 1985 Nov;18(5):537-43.

139. Olanow CW, Stern MB, Sethi K. The scientific and clinical basis for the treatment of Parkinson's disease. Neurology. 2009 May;72(21 Suppl 4):S1-136.

140. Poewe W. Clinical and pathophysiologic aspects of late levodopa failure. Neurology. 1993 Dec;43(12 Suppl 6):S28-30.

141. Ahlskog JE, Muenter MD. Frequency of levodopa-related dyskinesias and motor fluctuations as estimated from the cumulative literature. Mov Disord. 2001 May;16(3):448-58.

142. Shoulson I, Glaubiger GA, Chase TN. On-off response. Clinical and biochemical correlations during oral and intravenous levodopa administration in parkinsonian patients. Neurology. 1975 Dec;25(12):1144-8.

143. Olanow CW, Obeso JA, Stocchi F. Drug insight: Continuous dopaminergic stimulation in the treatment of Parkinson's disease. Nat Clin Pract Neurol. 2006 Jul;2(7):382-92.

144. Obeso JA, Rodriguez-Oroz M, Marin C, Alonso F, Zamarbide I, Lanciego JL, et al. The origin of motor fluctuations in Parkinson's disease: importance of dopaminergic innervation and basal ganglia circuits. Neurology. 2004 Jan;62(1 Suppl 1):S17-30.

145. Lees AJ, Hardy J, Revesz T. Parkinson's disease. Lancet. 2009 Jun;373(9680):2055-66.

146. Rinne UK, Siirtola T, Sonninen V. L-deprenyl treatment of on-off phenomena in Parkinson's disease. J Neural Transm. 1978 Jan;43(3-4):253-62.

147. Yasar S, Justinova Z, Lee SH, Stefanski R, Goldberg SR, Tanda G. Metabolic transformation plays a primary role in the psychostimulant-like discriminativestimulus effects of selegiline. J Pharmacol Exp Ther. 2006 Apr;317(1):387-94.

148. Poewe W. Treatments for Parkinson's disease-past achievements and current clinical needs. Neurology. 2009 Feb;72(7 Suppl):S65-73.

149. Voon V, Kubu C, Krack P, Houeto JL, Troster AI. Deep brain stimulation: neuropsychological and neuropsychiatric issues. Mov Disord. 2006 Jun;21 Suppl 14:S305-27.

150. Madrazo I, Leon V, Torres C, Aguilera MC, Varela G, Alvarez F, et al. Transplantation of fetal substantia nigra and adrenal medulla to the caudate nucleus in two patients with Parkinson's disease. N Engl J Med. 1988 Jan;318(1):51.

151. Lindvall O, Rehncrona S, Brundin P, Gustavii B, Astedt B, Widner H, et al. Human fetal dopamine neurons grafted into the striatum in two patients with severe Parkinson's disease. A detailed account of methodology and a 6-month follow-up. Arch Neurol. 1989 Jun;46(6):615-31. 
152. Piccini P, Brooks DJ, Bjorklund A, Gunn RN, Grasby PM, Rimoldi O, et al. Dopamine release from nigral transplants visualized in vivo in a Parkinson's patient. Nat Neurosci. 1999 Dec;2(12):1137-40.

153. Hauser RA, Freeman TB, Snow BJ, Nauert M, Gauger L, Kordower JH, et al. Long-term evaluation of bilateral fetal nigral transplantation in Parkinson's disease. Arch Neurol. 1999 Feb;56(2):179-87.

154. Wijeyekoon R, Barker RA. Cell replacement therapy for Parkinson's disease. Biochim Biophys Acta. 2009 Jul;1792(7):688-702.

155. Mendez I, Vinuela A, Astradsson A, Mukhida K, Hallett P, Robertson H, et al. Dopamine neurons implanted into people with Parkinson's disease survive without pathology for 14 years. Nat Med. 2008 May;14(5):507-9.

156. Masliah E, Rockenstein E, Veinbergs I, Mallory M, Hashimoto M, Takeda A, et al. Dopaminergic loss and inclusion body formation in alpha-synuclein mice: implications for neurodegenerative disorders. Science. 2000 Feb;287(5456):12659.

157. Thiruchelvam MJ, Powers JM, Cory-Slechta DA, Richfield EK. Risk factors for dopaminergic neuron loss in human alpha-synuclein transgenic mice. Eur J Neurosci. 2004 Feb;19(4):845-54.

158. Nuber S, Petrasch-Parwez E, Winner B, Winkler J, von Horsten S, Schmidt T, et al. Neurodegeneration and motor dysfunction in a conditional model of Parkinson's disease. J Neurosci. 2008 Mar;28(10):2471-84.

159. Drobysheva D, Ameel K, Welch B, Ellison E, Chaichana K, Hoang B, et al. An optimized method for histological detection of dopaminergic neurons in Drosophila melanogaster. J Histochem Cytochem. 2008 Dec;56(12):1049-63.

160. Mata IF, Wedemeyer WJ, Farrer MJ, Taylor JP, Gallo KA. LRRK2 in Parkinson's disease: protein domains and functional insights. Trends Neurosci. 2006 May;29(5):286-93.

161. Nichols WC, Pankratz N, Hernandez D, Paisan-Ruiz C, Jain S, Halter CA, et al. Genetic screening for a single common LRRK2 mutation in familial Parkinson's disease. Lancet. 2005 Jan;365(9457):410-2.

162. Lim KL, Ng CH. Genetic models of Parkinson's disease. Biochim Biophys Acta. 2009 Jul;1792(7):604-15.

163. Lee SB, Kim W, Lee S, Chung J. Loss of LRRK2/PARK8 induces degeneration of dopaminergic neurons in Drosophila. Biochem Biophys Res Commun. 2007 Jun;358(2):534-9.

164. Kitada T, Asakawa S, Hattori N, Matsumine H, Yamamura Y, Minoshima S, et al. Mutations in the parkin gene cause autosomal recessive juvenile parkinsonism. Nature. 1998 Apr;392(6676):605-8.

165. Whitworth AJ, Theodore DA, Greene JC, Benes H, Wes PD, Pallanck LJ. Increased glutathione S-transferase activity rescues dopaminergic neuron loss in a Drosophila model of Parkinson's disease. Proc Natl Acad Sci U S A. 2005 May;102(22):8024-9.

166. Shimohama S, Sawada H, Kitamura Y, Taniguchi T. Disease model: Parkinson's disease. Trends Mol Med. 2003 Aug;9(8):360-5. 
167. Hamre K, Tharp R, Poon K, Xiong X, Smeyne RJ. Differential strain susceptibility following 1-methyl-4-phenyl-1,2,3,6-tetrahydropyridine (MPTP) administration acts in an autosomal dominant fashion: quantitative analysis in seven strains of Mus musculus. Brain Res. 1999 May;828(1-2):91-103.

168. Przedborski S, Jackson-Lewis V, Naini AB, Jakowec M, Petzinger G, Miller R, et al. The parkinsonian toxin 1-methyl-4-phenyl-1,2,3,6-tetrahydropyridine (MPTP): a technical review of its utility and safety. J Neurochem. 2001 Mar;76(5):1265-74.

169. Betarbet R, Sherer TB, MacKenzie G, Garcia-Osuna M, Panov AV, Greenamyre JT. Chronic systemic pesticide exposure reproduces features of Parkinson's disease. Nat Neurosci. 2000 Dec;3(12):1301-6.

170. Cicchetti F, Drouin-Ouellet J, Gross RE. Environmental toxins and Parkinson's disease: what have we learned from pesticide-induced animal models? Trends Pharmacol Sci. 2009 Sep;30(9):475-83.

171. Brooks AI, Chadwick CA, Gelbard HA, Cory-Slechta DA, Federoff HJ. Paraquat elicited neurobehavioral syndrome caused by dopaminergic neuron loss. Brain Res. 1999 Mar;823(1-2):1-10.

172. Zhang J, Fitsanakis VA, Gu G, Jing D, Ao M, Amarnath V, et al. Manganese ethylene-bis-dithiocarbamate and selective dopaminergic neurodegeneration in rat: a link through mitochondrial dysfunction. J Neurochem. 2003 Jan;84(2):33646.

173. Ferraz HB, Bertolucci PH, Pereira JS, Lima JG, Andrade LA. Chronic exposure to the fungicide maneb may produce symptoms and signs of CNS manganese intoxication. Neurology. 1988 Apr;38(4):550-3.

174. Thiruchelvam M, Richfield EK, Baggs RB, Tank AW, Cory-Slechta DA. The nigrostriatal dopaminergic system as a preferential target of repeated exposures to combined paraquat and maneb: implications for Parkinson's disease. J Neurosci. 2000 Dec;20(24):9207-14.

175. Thiruchelvam M, Brockel BJ, Richfield EK, Baggs RB, Cory-Slechta DA. Potentiated and preferential effects of combined paraquat and maneb on nigrostriatal dopamine systems: environmental risk factors for Parkinson's disease? Brain Res. 2000 Aug;873(2):225-34.

176. Menninger KA. Psychoses associated with influenza. Arch Neurol Psych. 1919 Jan;2:291-337.

177. Menninger KA. Influenza and schizophrenia. An analysis of post-influenzal "dementia precox" as of 1918 and five years later. Am J Psych. 1926 Jan;5:469529.

178. Chandesris MO, Bernit E, Schleinitz N, Nicolino C, Bensa P, Tammam D, et al. A case of Influenza virus encephalitis in south of France. Rev Med Interne. 2004 Jan;25(1):78-82.

179. Dale RC, Church AJ, Surtees RA, Lees AJ, Adcock JE, Harding B, et al. Encephalitis lethargica syndrome: 20 new cases and evidence of basal ganglia autoimmunity. Brain. 2004 Jan;127(Pt 1):21-33.

180. Tanaka H, Park CH, Ninomiya A, Ozaki H, Takada A, Umemura T, et al. Neurotropism of the 1997 Hong Kong H5N1 influenza virus in mice. Vet Microbiol. 2003 Aug;95(1-2):1-13. 
181. Lipatov AS, Krauss S, Guan Y, Peiris M, Rehg JE, Perez DR, et al.

Neurovirulence in mice of H5N1 influenza virus genotypes isolated from Hong Kong poultry in 2001. J Virol. 2003 Mar;77(6):3816-23.

182. Ryan MM, Procopis PG, Ouvrier RA. Influenza A encephalitis with movement disorder. Pediatr Neurol. 1999 Sep;21(3):669-73.

183. Lamb RA, Takeda M. Death by influenza virus protein. Nat Med. 2001 Dec;7(12):1286-8.

184. Cheung TK, Poon LL. Biology of influenza a virus. Ann N Y Acad Sci. 2007 Apr;1102:1-25.

185. Monto AS, Gravenstein S, Elliott M, Colopy M, Schweinle J. Clinical signs and symptoms predicting influenza infection. Arch Intern Med. 2000 Nov;160(21):3243-7.

186. Nicholson KG, Wood JM, Zambon M. Influenza. Lancet. 2003 Nov;362(9397):1733-45.

187. Martin CM, Kunin CM, Gottlieb LS, Barnes MW, Liu C, Finland M. Asian influenza A in Boston, 1957-1958. I. Observations in thirty-two influenzaassociated fatal cases. AMA Arch Intern Med. 1959 Apr;103(4):515-31.

188. Hayase Y, Tobita K. Influenza virus and neurological diseases. Psychiatry Clin Neurosci. 1997 Aug;51(4):181-4.

189. Guarner J, Paddock CD, Shieh WJ, Packard MM, Patel M, Montague JL, et al. Histopathologic and immunohistochemical features of fatal influenza virus infection in children during the 2003-2004 season. Clin Infect Dis. 2006 Jul;43(2):132-40.

190. Chen J, Lee KH, Steinhauer DA, Stevens DJ, Skehel JJ, Wiley DC. Structure of the hemagglutinin precursor cleavage site, a determinant of influenza pathogenicity and the origin of the labile conformation. Cell. 1998 Oct;95(3):40917.

191. Skehel JJ, Bayley PM, Brown EB, Martin SR, Waterfield MD, White JM, et al. Changes in the conformation of influenza virus hemagglutinin at the $\mathrm{pH}$ optimum of virus-mediated membrane fusion. Proc Natl Acad Sci U S A. 1982 Feb;79(4):968-72.

192. Webby RJ, Perez DR, Coleman JS, Guan Y, Knight JH, Govorkova EA, et al. Responsiveness to a pandemic alert: use of reverse genetics for rapid development of influenza vaccines. Lancet. 2004 Apr;363(9415):1099-103.

193. Jin H, Leser GP, Zhang J, Lamb RA. Influenza virus hemagglutinin and neuraminidase cytoplasmic tails control particle shape. Embo J. 1997 Mar;16(6):1236-47.

194. Mitnaul LJ, Castrucci MR, Murti KG, Kawaoka Y. The cytoplasmic tail of influenza A virus neuraminidase (NA) affects NA incorporation into virions, virion morphology, and virulence in mice but is not essential for virus replication. J Virol. 1996 Feb;70(2):873-9.

195. Bui M, Whittaker G, Helenius A. Effect of M1 protein and low $\mathrm{pH}$ on nuclear transport of influenza virus ribonucleoproteins. J Virol. 1996 Dec;70(12):8391401. 
196. Martin K, Helenius A. Nuclear transport of influenza virus ribonucleoproteins: the viral matrix protein (M1) promotes export and inhibits import. Cell. 1991 Oct;67(1):117-30.

197. Ulmanen I, Broni BA, Krug RM. Role of two of the influenza virus core P proteins in recognizing cap 1 structures $(\mathrm{m} 7 \mathrm{GpppNm})$ on RNAs and in initiating viral RNA transcription. Proc Natl Acad Sci U S A. 1981 Dec;78(12):7355-9.

198. Webster RG. The importance of animal influenza for human disease. Vaccine. 2002 May;20 Suppl 2:S16-20.

199. Whittaker G, Bui M, Helenius A. Nuclear trafficking of influenza virus ribonuleoproteins in heterokaryons. J Virol. 1996 May;70(5):2743-56.

200. Cheung CY, Poon LL, Lau AS, Luk W, Lau YL, Shortridge KF, et al. Induction of proinflammatory cytokines in human macrophages by influenza A (H5N1) viruses: a mechanism for the unusual severity of human disease? Lancet. 2002 Dec;360(9348):1831-7.

201. Neumann G, Hughes MT, Kawaoka Y. Influenza A virus NS2 protein mediates vRNP nuclear export through NES-independent interaction with hCRM1. Embo J. 2000 Dec;19(24):6751-8.

202. Hay AJ. The virus genome and its replication Oxford: Blackwell Science Ltd.; 1998.

203. Plotch SJ, Bouloy M, Ulmanen I, Krug RM. A unique cap(m7GpppXm)dependent influenza virion endonuclease cleaves capped RNAs to generate the primers that initiate viral RNA transcription. Cell. 1981 Mar;23(3):847-58.

204. Reid AH, McCall S, Henry JM, Taubenberger JK. Experimenting on the past: the enigma of von Economo's encephalitis lethargica. J Neuropathol Exp Neurol. 2001 Jul;60(7):663-70.

205. Olgar S, Ertugrul T, Nisli K, Aydin K, Caliskan M. Influenza a-associated acute necrotizing encephalopathy. Neuropediatrics. 2006 Jun;37(3):166-8.

206. Drago L, Attia MW, DePiero A, Bean C. Influenza A-associated meningoencephalitis. Pediatr Emerg Care. 2005 Jul;21(7):437-9.

207. de Jong MD, Bach VC, Phan TQ, Vo MH, Tran TT, Nguyen BH, et al. Fatal avian influenza $\mathrm{A}(\mathrm{H} 5 \mathrm{~N} 1)$ in a child presenting with diarrhea followed by coma. N Engl J Med. 2005 Feb;352(7):686-91.

208. Togashi T, Matsuzono Y, Narita M, Morishima T. Influenza-associated acute encephalopathy in Japanese children in 1994-2002. Virus Res. 2004 Jul;103(12):75-8.

209. Iijima H, Wakasugi K, Ayabe M, Shoji H, Abe T. A case of adult influenza A virus-associated encephalitis: magnetic resonance imaging findings. $\mathrm{J}$ Neuroimaging. 2002 Jul;12(3):273-5.

210. Mihara M, Utsugisawa K, Konno S, Tohgi H. Isolated lesions limited to the bilateral substantia nigra on MRI associated with influenza A infection. Eur Neurol. 2001 Jan;45(4):290-1.

211. Bayer WH. Influenza B encephalitis. West J Med. 1987 Oct;147(4):466.

212. Lawrence GH, Jones RA. Case report; encephalitis as a complication of the influenza. Mo Med. 1957 Dec;54(12):1164.

213. Taubenberger JK, Morens DM. 1918 Influenza: the mother of all pandemics. Emerg Infect Dis. 2006 Jan;12(1):15-22. 
214. Cruchet JR. Quarante cas d'encéphalo-myélite subaiguë. . Bulletins et Mémoires de la Société Médicale des Hôpitaux de Paris. 1917 Apr;41:614-6.

215. Vilensky JA, Gilman S. Encephalitis lethargica: could this disease be recognised if the epidemic recurred? Practical Neurology. 2006 Jan;6:360-7.

216. Bao Y, Bolotov P, Dernovoy D, Kiryutin B, Zaslavsky L, Tatusova T, et al. The influenza virus resource at the National Center for Biotechnology Information. $\mathrm{J}$ Virol. 2008 Jan;82(2):596-601.

217. Wong KT, Allen IV, McQuaid S, McConnell R. An immunohistochemical study of neurofibrillary tangle formation in post-encephalitic Parkinsonism. Clin Neuropathol. 1996 Jan;15(1):22-5.

218. Taubenberger JK, Reid AH, Janczewski TA, Fanning TG. Integrating historical, clinical and molecular genetic data in order to explain the origin and virulence of the 1918 Spanish influenza virus. Philos Trans R Soc Lond B Biol Sci. 2001 Dec;356(1416):1829-39.

219. Poskanzer DC, Schwab RS. Cohort Analysis of Parkinson's Syndrome: Evidence for a Single Etiology Related to Subclinical Infection About 1920. J Chronic Dis. 1963 Sep;16:961-73.

220. Maurizi CP. Postencephalitic Parkinson's disease, amyotrophic lateral sclerosis on Guam and influenza revisited: focusing on neurofibrillary tangles and the trail of tau. Med Hypotheses. 2002 Mar;58(3):198-202.

221. Klopfleisch R, Werner O, Mundt E, Harder T, Teifke JP. Neurotropism of highly pathogenic avian influenza virus A/chicken/Indonesia/2003 (H5N1) in experimentally infected pigeons (Columbia livia f. domestica). Vet Pathol. 2006 Jul;43(4):463-70.

222. Rigoni M, Shinya K, Toffan A, Milani A, Bettini F, Kawaoka Y, et al. Pneumoand neurotropism of avian origin Italian highly pathogenic avian influenza H7N1 isolates in experimentally infected mice. Virology. $2007 \mathrm{Jul} ; 364(1): 28-35$.

223. McCall S, Henry JM, Reid AH, Taubenberger JK. Influenza RNA not detected in archival brain tissues from acute encephalitis lethargica cases or in postencephalitic Parkinson cases. J Neuropathol Exp Neurol. 2001 Jul;60(7):696704.

224. Elizan TS, Casals J. Astrogliosis in von Economo's and postencephalitic Parkinson's diseases supports probable viral etiology. J Neurol Sci. 1991 Oct;105(2):131-4.

225. Mori I, Imai Y, Kohsaka S, Kimura Y. Upregulated expression of Iba1 molecules in the central nervous system of mice in response to neurovirulent influenza A virus infection. Microbiol Immunol. 2000 Jan;44(8):729-35.

226. Dalldorf G. The coxsackie group of viruses. Science. 1949 Jan;110:594.

227. Dalldorf G, Sickles GM. An Unidentified, Filtrable Agent Isolated From the Feces of Children With Paralysis. Science. 1948 Jan;108:61-2.

228. Control CfDPa. Enterovirus surveillance--United States, 1997-1999. MMWR Morb Mortal Wkly Rep. 2000 Oct;49(40):913-6.

229. Muir P, van Loon AM. Enterovirus infections of the central nervous system. Intervirology. 1997 Jan;40(2-3):153-66.

230. Tam PE. Coxsackievirus myocarditis: interplay between virus and host in the pathogenesis of heart disease. Viral Immunol. 2006 Jun;19(2):133-46. 
231. Hirschman SZ, Hammer GS. Coxsackie virus myopericarditis. A microbiological and clinical review. Am J Cardiol. 1974 Aug;34(2):224-32.

232. Turell MJ, O'Guinn ML, Wasieloski LP, Jr., Dohm DJ, Lee WJ, Cho HW, et al. Isolation of Japanese encephalitis and Getah viruses from mosquitoes (Diptera: Culicidae) collected near Camp Greaves, Gyonggi Province, Republic of Korea, 2000. J Med Entomol. 2003 Jul;40(4):580-4.

233. Mackenzie JS, Gubler DJ, Petersen LR. Emerging flaviviruses: the spread and resurgence of Japanese encephalitis, West Nile and dengue viruses. Nat Med. 2004 Dec;10(12 Suppl):S98-109.

234. Luby JP, Sulkin SE, Sanford JP. The epidemiology of St. Louis encephalitis: a review. Annu Rev Med. 1969 Jan;20:329-50.

235. Solomon T, Winter PM. Neurovirulence and host factors in flavivirus encephalitis--evidence from clinical epidemiology. Arch Virol Suppl. 2004 $\operatorname{Jan}(18): 161-70$.

236. Diagana M, Preux PM, Dumas M. Japanese encephalitis revisited. J Neurol Sci. 2007 Nov;262(1-2):165-70.

237. Ishii T, Matsushita M, Hamada S. Characteristic residual neuropathological features of Japanese B encephalitis. Acta Neuropathol. 1977 Jun;38(3):181-6.

238. Tiroumourougane SV, Raghava P, Srinivasan S. Japanese viral encephalitis. Postgrad Med J. 2002 Apr;78(918):205-15.

239. Abe T, Kojima K, Shoji H, Tanaka N, Fujimoto K, Uchida M, et al. Japanese encephalitis. J Magn Reson Imaging. 1998 Jul;8(4):755-61.

240. Sarngadharan MG, DeVico AL, Bruch L, Schupbach J, Gallo RC. HTLV-III: the etiologic agent of AIDS. Princess Takamatsu Symp. 1984 Jan;15:301-8.

241. Mattos JP, Rosso AL, Correa RB, Novis SA. Movement disorders in 28 HIVinfected patients. Arq Neuropsiquiatr. 2002 Sep;60(3-A):525-30.

242. Tse W, Cersosimo MG, Gracies JM, Morgello S, Olanow CW, Koller W. Movement disorders and AIDS: a review. Parkinsonism Relat Disord. 2004 Aug;10(6):323-34.

243. Krebs FC, Ross H, McAllister J, Wigdahl B. HIV-1-associated central nervous system dysfunction. Adv Pharmacol. 2000 Jan;49:315-85.

244. Budka H. Human immunodeficiency virus (HIV) envelope and core proteins in CNS tissues of patients with the acquired immune deficiency syndrome (AIDS). Acta Neuropathol. 1990;79(6):611-9.

245. Brack-Werner R. Astrocytes: HIV cellular reservoirs and important participants in neuropathogenesis. Aids. 1999 Jan;13(1):1-22.

246. Bell JE. The neuropathology of adult HIV infection. Rev Neurol (Paris). 1998 Dec;154(12):816-29.

247. Paul R, Cohen R, Navia B, Tashima K. Relationships between cognition and structural neuroimaging findings in adults with human immunodeficiency virus type-1. Neurosci Biobehav Rev. 2002 May;26(3):353-9.

248. Aylward EH, Henderer JD, McArthur JC, Brettschneider PD, Harris GJ, Barta PE, et al. Reduced basal ganglia volume in HIV-1-associated dementia: results from quantitative neuroimaging. Neurology. 1993 Oct;43(10):2099-104. 
249. Castelo JM, Courtney MG, Melrose RJ, Stern CE. Putamen hypertrophy in nondemented patients with human immunodeficiency virus infection and cognitive compromise. Arch Neurol. 2007 Sep;64(9):1275-80.

250. Hawkins CP, McLaughlin JE, Kendall BE, McDonald WI. Pathological findings correlated with MRI in HIV infection. Neuroradiology. 1993 Jan;35(4):264-8.

251. Kodama T, Numaguchi Y, Gellad FE, Sadato N. High signal intensity of both putamina in patients with HIV infection. Neuroradiology. 1991 Jan;33(4):362-3.

252. Luttmann S, Husstedt IW, Schuierer G, Kuhlenbaumer G, Stodieck S, Riedasch $\mathrm{M}$, et al. High-signal lesions in the midbrain on T1-weighted MRI in an HIVinfected patient. Neuroradiology. 1997 Feb;39(2):136-8.

253. Rottenberg DA, Moeller JR, Strother SC, Sidtis JJ, Navia BA, Dhawan V, et al. The metabolic pathology of the AIDS dementia complex. Ann Neurol. 1987 Dec;22(6):700-6.

254. Dybing JK, Schultz-Cherry S, Swayne DE, Suarez DL, Perdue ML. Distinct pathogenesis of hong kong-origin H5N1 viruses in mice compared to that of other highly pathogenic H5 avian influenza viruses. J Virol. 2000 Feb;74(3):1443-50.

255. WHO. Cumulative number of confirmed human cases of avian influenza A/(H5N1) reported to WHO. 2010 [accessed 2010 May]; Available from: http://www.who.int/csr/disease/avian influenza/country/cases table 2010 $05 \quad 06$ len/index.html

256. Rimmelzwaan GF, van Riel D, Baars M, Bestebroer TM, van Amerongen G, Fouchier RA, et al. Influenza A virus (H5N1) infection in cats causes systemic disease with potential novel routes of virus spread within and between hosts. Am J Pathol. 2006 Jan;168(1):176-83.

257. Smith GJ, Naipospos TS, Nguyen TD, de Jong MD, Vijaykrishna D, Usman TB, et al. Evolution and adaptation of H5N1 influenza virus in avian and human hosts in Indonesia and Vietnam. Virology. 2006 Jul;350(2):258-68.

258. Fodor E, Brownlee GG. Influenza virus replication. Amsterdam: Elsevier; 2002.

259. Staeheli P, Grob R, Meier E, Sutcliffe JG, Haller O. Influenza virus-susceptible mice carry Mx genes with a large deletion or a nonsense mutation. Mol Cell Biol. 1988 Oct;8(10):4518-23.

260. Horisberger MA. Interferons, Mx genes, and resistance to influenza virus. Am J Respir Crit Care Med. 1995 Oct;152(4 Pt 2):S67-71.

261. Engelhardt OG, Ullrich E, Kochs G, Haller O. Interferon-induced antiviral Mx1 GTPase is associated with components of the SUMO-1 system and promyelocytic leukemia protein nuclear bodies. Exp Cell Res. 2001 Dec;271(2):286-95.

262. Aronsson F, Robertson B, Ljunggren HG, Kristensson K. Invasion and persistence of the neuroadapted influenza virus A/WSN/33 in the mouse olfactory system. Viral Immunol. 2003 May;16(3):415-23.

263. Iwasaki T, Itamura S, Nishimura H, Sato Y, Tashiro M, Hashikawa T, et al. Productive infection in the murine central nervous system with avian influenza virus A (H5N1) after intranasal inoculation. Acta Neuropathol (Berl). 2004 Dec;108(6):485-92.

264. Matsuda K, Park CH, Sunden Y, Kimura T, Ochiai K, Kida H, et al. The vagus nerve is one route of transneural invasion for intranasally inoculated influenza a virus in mice. Vet Pathol. 2004 Mar;41(2):101-7. 
265. Reinacher M, Bonin J, Narayan O, Scholtissek C. Pathogenesis of neurovirulent influenza A virus infection in mice. Route of entry of virus into brain determines infection of different populations of cells. Lab Invest. 1983 Dec;49(6):686-92.

266. Shinya K, Shimada A, Ito T, Otsuki K, Morita T, Tanaka H, et al. Avian influenza virus intranasally inoculated infects the central nervous system of mice through the general visceral afferent nerve. Arch Virol. 2000 Oct;145(1):187-95.

267. Takahashi M, Yamada T, Nakajima S, Nakajima K, Yamamoto T, Okada H. The substantia nigra is a major target for neurovirulent influenza A virus. J Exp Med. 1995 Jun;181(6):2161-9.

268. Park CH, Ishinaka M, Takada A, Kida H, Kimura T, Ochiai K, et al. The invasion routes of neurovirulent A/Hong Kong/483/97 (H5N1) influenza virus into the central nervous system after respiratory infection in mice. Arch Virol. 2002 Jul;147(7):1425-36.

269. Gambotto A, Barratt-Boyes SM, de Jong MD, Neumann G, Kawaoka Y. Human infection with highly pathogenic H5N1 influenza virus. Lancet. 2008 Apr;371(9622):1464-75.

270. Jang H, Boltz D, Sturm-Ramirez K, Shepherd KR, Jiao Y, Webster R, et al. Highly pathogenic H5N1 influenza virus can enter the central nervous system and induce neuroinflammation and neurodegeneration. Proc Natl Acad Sci U S A. 2009, Aug; 106(33):14063-8

271. Reed LJ, Muench H. A simple method of estimating 50 per cent end-points. Amer J Hygiene. 1938 Jan(27):493-7.

272. van Wyke KL, Hinshaw VS, Bean WJ, Jr., Webster RG. Antigenic variation of influenza A virus nucleoprotein detected with monoclonal antibodies. J Virol. $1980 \mathrm{Jul} ; 35(1): 24-30$.

273. Paxinos G, Franklin KBJ. The Mouse Brain in Stereotaxic Coordinates. San Diego: Academic Press; 2001.

274. Chen SC, Kochan JP, Campfield LA, Burn P, Smeyne RJ. Splice variants of the OB receptor gene are differentially expressed in brain and peripheral tissues of mice. J Recept Signal Transduct Res. 1999 Jan;19(1-4):245-66.

275. Faherty CJ, Xanthoudakis S, Smeyne RJ. Caspase-3-dependent neuronal death in the hippocampus following kainic acid treatment. Brain Res Mol Brain Res. 1999 Jan;70(1):159-63.

276. Ajioka I, Martins RA, Bayazitov IT, Donovan S, Johnson DA, Frase S, et al. Differentiated horizontal interneurons clonally expand to form metastatic retinoblastoma in mice. Cell. 2007 Oct;131(2):378-90.

277. Malin SA, Davis BM, Molliver DC. Production of dissociated sensory neuron cultures and considerations for their use in studying neuronal function and plasticity. Nat Protoc. 2007 Dec;2(1):152-60.

278. Liu WW, Goodhouse J, Jeon NL, Enquist LW. A microfluidic chamber for analysis of neuron-to-cell spread and axonal transport of an alpha-herpesvirus. PLoS One. 2008 Jan;3(6):2382.

279. West MJ, Slomianka L, Gundersen HJ. Unbiased stereological estimation of the total number of neurons in thesubdivisions of the rat hippocampus using the optical fractionator. Anat Rec. 1991 Dec;231(4):482-97. 
280. Baquet ZC, Williams D, Brody J, Smeyne RJ. A comparison of model-based (2D) and design-based (3D) stereological methods for estimating cell number in the substantia nigra pars compacta $(\mathrm{SNpc})$ of the $\mathrm{C} 57 \mathrm{BL} / 6 \mathrm{~J}$ mouse. Neuroscience. $2009 \mathrm{Jul} ; 161(4): 1082-90$.

281. Ilyushina NA, Hay A, Yilmaz N, Boon AC, Webster RG, Govorkova EA. Oseltamivir-ribavirin combination therapy for highly pathogenic H5N1 influenza virus infection in mice. Antimicrob Agents Chemother. 2008 Nov;52(11):3889-97.

282. Hollis JH, Lightman SL, Lowry CA. Integration of systemic and visceral sensory information by medullary catecholaminergic systems during peripheral inflammation. Ann N Y Acad Sci. 2004 Jun;1018:71-5.

283. Wood JM, Robertson JS. From lethal virus to life-saving vaccine: developing inactivated vaccines for pandemic influenza. Nat Rev Microbiol. 2004 Oct;2(10):842-7.

284. Schmued LC, Stowers CC, Scallet AC, Xu L. Fluoro-Jade C results in ultra high resolution and contrast labeling of degenerating neurons. Brain Res. 2005 Feb;1035(1):24-31.

285. Liu B, Hong JS. Role of microglia in inflammation-mediated neurodegenerative diseases: mechanisms and strategies for therapeutic intervention. J Pharmacol Exp Ther. 2003 Jan;304(1):1-7.

286. Mosley RL, Benner EJ, Kadiu I, Thomas M, Boska MD, Hasan K, et al. Neuroinflammation, Oxidative Stress and the Pathogenesis of Parkinson's Disease. Clin Neurosci Res. 2006 Dec;6(5):261-81.

287. Lo KC, Geddes JF, Daniels RS, Oxford JS. Lack of detection of influenza genes in archived formalin-fixed, paraffin wax-embedded brain samples of encephalitis lethargica patients from 1916 to 1920. Virchows Arch. 2003 Jun;442(6):591-6.

288. Weidong L, Shen C, Jankovic J. Etiopathogenesis of Parkinson's disease: a new beginning? Neuroscientist. 2009 Feb;15(1):28-35.

289. Steece-Collier K, Maries E, Kordower JH. Etiology of Parkinson's disease: Genetics and environment revisited. Proc Natl Acad Sci U S A. 2002 Oct;99(22):13972-4.

290. Smith DJ, Stevens ME, Sudanagunta SP, Bronson RT, Makhinson M, Watabe $\mathrm{AM}$, et al. Functional screening of $2 \mathrm{Mb}$ of human chromosome 21q22.2 in transgenic mice implicates minibrain in learning defects associated with Down syndrome. Nature Genetics. 1997 Jan;16(1):28-36.

291. Ralph F. Józefowicz JJM, James M. Powers. Microglial activation formation of pleomorphic microglia and macrophages. 2000 [accessed 2010 May]; Available from: http://www.urmc.rochester.edu/neuroslides/slide004.html

292. Smeyne M, Boyd J, Raviie Shepherd K, Jiao Y, Pond BB, Hatler M, et al. GSTpi expression mediates dopaminergic neuron sensitivity in experimental parkinsonism. Proc Natl Acad Sci U S A. 2007 Feb;104(6):1977-82.

293. Ransohoff RM, Perry VH. Microglial physiology: unique stimuli, specialized responses. Annu Rev Immunol. 2009 Jan;27:119-45.

294. Harry GJ, Kraft AD. Neuroinflammation and microglia: considerations and approaches for neurotoxicity assessment. Expert Opin Drug Metab Toxicol. 2008 Oct;4(10):1265-77. 
295. Graeber MB, Streit WJ. Microglia: biology and pathology. Acta Neuropathol. 2010 Jan;119(1):89-105.

296. Perry VH, Newman TA, Cunningham C. The impact of systemic infection on the progression of neurodegenerative disease. Nat Rev Neurosci. 2003 Feb;4(2):10312.

297. Yuen KY, Wong SS. Human infection by avian influenza A H5N1. Hong Kong Med J. 2005 Jun;11(3):189-99.

298. Opal SM, DePalo VA. Anti-inflammatory cytokines. Chest. 2000 Apr;117(4):1162-72.

299. Fernandez EJ, Lolis E. Structure, function, and inhibition of chemokines. Annu Rev Pharmacol Toxicol. 2002 Jun;42:469-99.

300. Metcalf $\mathrm{D}$. The granulocyte-macrophage colony-stimulating factors. Science. 1985 Jul;229(4708):16-22.

301. Hirsch EC, Hunot S. Neuroinflammation in Parkinson's disease: a target for neuroprotection? Lancet Neurol. 2009 Apr;8(4):382-97.

302. Glass CK, Saijo K, Winner B, Marchetto MC, Gage FH. Mechanisms underlying inflammation in neurodegeneration. Cell. 2010 Mar 19;140(6):918-34.

303. Zhou C, Huang Y, Przedborski S. Oxidative stress in Parkinson's disease: a mechanism of pathogenic and therapeutic significance. Ann N Y Acad Sci. 2008 Dec;1147:93-104.

304. Sanchez-Guajardo V, Febbraro F, Kirik D, Romero-Ramos M. Microglia acquire distinct activation profiles depending on the degree of alpha-synuclein neuropathology in a rAAV based model of Parkinson's disease. PLoS One. 2010 Jan;5(1):Epub ahead of print.

305. Reynolds AD, Stone DK, Mosley RL, Gendelman HE. Nitrated alpha-synucleininduced alterations in microglial immunity are regulated by $\mathrm{CD} 4+\mathrm{T}$ cell subsets. J Immunol. 2009 Apr;182(7):4137-49.

306. Su X, Maguire-Zeiss KA, Giuliano R, Prifti L, Venkatesh K, Federoff HJ. Synuclein activates microglia in a model of Parkinson's disease. Neurobiol Aging. 2008 Nov;29(11):1690-701.

307. Zhang W, Wang T, Pei Z, Miller DS, Wu X, Block ML, et al. Aggregated alphasynuclein activates microglia: a process leading to disease progression in Parkinson's disease. Faseb J. 2005 Apr;19(6):533-42.

308. Minghetti L, Ajmone-Cat MA, De Berardinis MA, De Simone R. Microglial activation in chronic neurodegenerative diseases: roles of apoptotic neurons and chronic stimulation. Brain Res Brain Res Rev. 2005 Apr;48(2):251-6.

309. Rojo AI, Innamorato NG, Martin-Moreno AM, De Ceballos ML, Yamamoto M, Cuadrado A. Nrf2 regulates microglial dynamics and neuroinflammation in experimental Parkinson's disease. Glia. 2010 Apr;58(5):588-98.

310. Kuhn DM, Francescutti-Verbeem DM, Thomas DM. Dopamine quinones activate microglia and induce a neurotoxic gene expression profile: relationship to methamphetamine-induced nerve ending damage. Ann N Y Acad Sci. 2006 Aug; 1074:31-41.

311. Liu M, Cai T, Zhao F, Zheng G, Wang Q, Chen Y, et al. Effect of microglia activation on dopaminergic neuronal injury induced by manganese, and its possible mechanism. Neurotox Res. 2009 Jul;16(1):42-9. 
312. Peng J, Stevenson FF, Oo ML, Andersen JK. Iron-enhanced paraquat-mediated dopaminergic cell death due to increased oxidative stress as a consequence of microglial activation. Free Radic Biol Med. 2009 Jan;46(2):312-20.

313. Gao HM, Hong JS. Why neurodegenerative diseases are progressive: uncontrolled inflammation drives disease progression. Trends Immunol. 2008 Aug;29(8):35765.

314. Hu X, Zhang D, Pang H, Caudle WM, Li Y, Gao H, et al. Macrophage antigen complex-1 mediates reactive microgliosis and progressive dopaminergic neurodegeneration in the MPTP model of Parkinson's disease. J Immunol. 2008 Nov;181(10):7194-204.

315. Qin L, Wu X, Block ML, Liu Y, Breese GR, Hong JS, et al. Systemic LPS causes chronic neuroinflammation and progressive neurodegeneration. Glia. 2007 Apr;55(5):453-62.

316. Ling Z, Zhu Y, Tong C, Snyder JA, Lipton JW, Carvey PM. Progressive dopamine neuron loss following supra-nigral lipopolysaccharide (LPS) infusion into rats exposed to LPS prenatally. Exp Neurol. 2006 Jun;199(2):499-512.

317. Wang G, Zhang J, Li W, Xin G, Su Y, Gao Y, et al. Apoptosis and proinflammatory cytokine responses of primary mouse microglia and astrocytes induced by human H1N1 and avian H5N1 influenza viruses. Cell Mol Immunol. 2008 Apr;5(2):113-20.

318. Yao L, Korteweg C, Hsueh W, Gu J. Avian influenza receptor expression in H5N1-infected and noninfected human tissues. Faseb J. 2008 Mar;22(3):733-40.

319. Yokota S, Imagawa T, Miyamae T, Ito S, Nakajima S, Nezu A, et al. Hypothetical pathophysiology of acute encephalopathy and encephalitis related to influenza virus infection and hypothermia therapy. Pediatr Int. 2000 Apr;42(2):197-203.

320. de Jong MD. H5N1 transmission and disease: observations from the frontlines. Pediatr Infect Dis J. 2008 Oct;27(10 Suppl):S54-6.

321. Tumpey TM, Basler CF, Aguilar PV, Zeng H, Solorzano A, Swayne DE, et al. Characterization of the reconstructed 1918 Spanish influenza pandemic virus. Science. 2005 Oct;310(5745):77-80.

322. Stevenson PG, Bangham CR, Hawke S. Recruitment, activation and proliferation of CD8+ memory T cells in an immunoprivileged site. Eur J Immunol. 1997 Dec;27(12):3259-68.

323. Stevenson PG, Hawke S, Bangham CR. Protection against lethal influenza virus encephalitis by intranasally primed CD8+ memory T cells. J Immunol. 1996 Oct;157(7):3065-73.

324. Paludan SR, Lovmand J, Ellermann-Eriksen S, Mogensen SC. Effect of IL-4 and IL-13 on IFN-gamma-induced production of nitric oxide in mouse macrophages infected with herpes simplex virus type 2. FEBS Lett. 1997 Sep;414(1):61-4.

325. Cash E, Minty A, Ferrara P, Caput D, Fradelizi D, Rott O. Macrophageinactivating IL-13 suppresses experimental autoimmune encephalomyelitis in rats. J Immunol. 1994 Nov;153(9):4258-67.

326. Bogdan C, Thuring H, Dlaska M, Rollinghoff M, Weiss G. Mechanism of suppression of macrophage nitric oxide release by IL-13: influence of the macrophage population. J Immunol. 1997 Nov;159(9):4506-13. 
327. Ruetten H, Thiemermann C. Interleukin-13 is a more potent inhibitor of the expression of inducible nitric oxide synthase in smooth muscle cells than in macrophages: a comparison with interleukin-4 and interleukin-10. Shock. 1997 Dec;8(6):409-14.

328. Shin WH, Lee DY, Park KW, Kim SU, Yang MS, Joe EH, et al. Microglia expressing interleukin-13 undergo cell death and contribute to neuronal survival in vivo. Glia. $2004 \mathrm{Apr} ; 46(2): 142-52$.

329. Park KW, Baik HH, Jin BK. IL-13-induced oxidative stress via microglial NADPH oxidase contributes to death of hippocampal neurons in vivo. J Immunol. 2009 Oct;183(7):4666-74.

330. Bissonnette CJ, Klegeris A, McGeer PL, McGeer EG. Interleukin 1alpha and interleukin 6 protect human neuronal SH-SY5Y cells from oxidative damage. Neurosci Lett. 2004 May;361(1-3):40-3.

331. Carlson NG, Wieggel WA, Chen J, Bacchi A, Rogers SW, Gahring LC. Inflammatory cytokines IL-1 alpha, IL-1 beta, IL-6, and TNF-alpha impart neuroprotection to an excitotoxin through distinct pathways. J Immunol. 1999 Oct;163(7):3963-8.

332. Petitto JM, Huang Z, Lo J, Streit WJ. IL-2 gene knockout affects T lymphocyte trafficking and the microglial response to regenerating facial motor neurons. J Neuroimmunol. 2003 Jan;134(1-2):95-103.

333. Viviani B, Gardoni F, Bartesaghi S, Corsini E, Facchi A, Galli CL, et al. Interleukin-1 beta released by gp120 drives neural death through tyrosine phosphorylation and trafficking of NMDA receptors. J Biol Chem. 2006 Oct;281(40):30212-22.

334. Orzylowska O, Oderfeld-Nowak B, Zaremba M, Januszewski S, Mossakowski M. Prolonged and concomitant induction of astroglial immunoreactivity of interleukin-1 beta and interleukin-6 in the rat hippocampus after transient global ischemia. Neurosci Lett. 1999 Mar;263(1):72-6.

335. Nguyen MD, D'Aigle T, Gowing G, Julien JP, Rivest S. Exacerbation of motor neuron disease by chronic stimulation of innate immunity in a mouse model of amyotrophic lateral sclerosis. J Neurosci. 2004 Feb;24(6):1340-9.

336. Goncalves J, Baptista S, Martins T, Milhazes N, Borges F, Ribeiro CF, et al. Methamphetamine-induced neuroinflammation and neuronal dysfunction in the mice hippocampus: preventive effect of indomethacin. Eur J Neurosci. 2010 Jan;31(2):315-26.

337. Mastroeni D, Grover A, Leonard B, Joyce JN, Coleman PD, Kozik B, et al. Microglial responses to dopamine in a cell culture model of Parkinson's disease. Neurobiol Aging. 2009 Nov;30(11):1805-17.

338. Smith TS, Parker WD, Jr., Bennett JP, Jr. L-dopa increases nigral production of hydroxyl radicals in vivo: potential L-dopa toxicity? Neuroreport. 1994 Apr;5(8):1009-11.

339. Miyazaki I, Asanuma M. Dopaminergic neuron-specific oxidative stress caused by dopamine itself. Acta Med Okayama. 2008 Jun;62(3):141-50.

340. Gao HM, Kotzbauer PT, Uryu K, Leight S, Trojanowski JQ, Lee VM. Neuroinflammation and oxidation/nitration of alpha-synuclein linked to dopaminergic neurodegeneration. J Neurosci. 2008 Jul;28(30):7687-98. 
341. Godbout JP, Johnson RW. Age and neuroinflammation: a lifetime of psychoneuroimmune consequences. Immunol Allergy Clin North Am. 2009 May;29(2):321-37.

342. Martyn CN. Infection in childhood and neurological diseases in adult life. Br Med Bull. 1997 Jan;53(1):24-39.

343. Gubareva LV, McCullers JA, Bethell RC, Webster RG. Characterization of influenza A/HongKong/156/97 (H5N1) virus in a mouse model and protective effect of zanamivir on H5N1 infection in mice. J Infect Dis. 1998 Dec;178(6):1592-6.

344. West MJ, Gundersen HJ. Unbiased stereological estimation of the number of neurons in the human hippocampus. J Comp Neurol. 1990 Jun;296(1):1-22.

345. Center PI. About paraquat. 2008 [accessed 2010 May]; Available from: http://www.paraquat.com

346. Jian XD, Sui H, Chu ZH, Zhang ZW, Kan BT, Zhang L, et al. Changes of serum cytokine caused by acute paraquat poisoning. Zhonghua Lao Dong Wei Sheng Zhi Ye Bing Za Zhi. 2007 Apr;25(4):230-2.

347. Richardson JR, Quan Y, Sherer TB, Greenamyre JT, Miller GW. Paraquat neurotoxicity is distinct from that of MPTP and rotenone. Toxicol Sci. 2005 Nov;88(1):193-201.

348. Bonneh-Barkay D, Reaney SH, Langston WJ, Di Monte DA. Redox cycling of the herbicide paraquat in microglial cultures. Brain Res Mol Brain Res. 2005 Mar; 134(1):52-6.

349. Salvemini D, Doyle TM, Cuzzocrea S. Superoxide, peroxynitrite and oxidative/nitrative stress in inflammation. Biochem Soc Trans. 2006 Nov;34(Pt 5):965-70.

350. Kuno R, Yoshida Y, Nitta A, Nabeshima T, Wang J, Sonobe Y, et al. The role of TNF-alpha and its receptors in the production of NGF and GDNF by astrocytes. Brain Res. 2006 Oct;1116(1):12-8.

351. Appel E, Kolman O, Kazimirsky G, Blumberg PM, Brodie C. Regulation of GDNF expression in cultured astrocytes by inflammatory stimuli. Neuroreport. 1997 Oct;8(15):3309-12.

352. Brodie C. Differential effects of Th1 and Th2 derived cytokines on NGF synthesis by mouse astrocytes. FEBS Lett. 1996 Sep;394(2):117-20.

353. Hattori A, Hayashi K, Kohno M. Tumor necrosis factor (TNF) stimulates the production of nerve growth factor in fibroblasts via the 55-kDa type $1 \mathrm{TNF}$ receptor. FEBS Lett. 1996 Jan;379(2):157-60.

354. Khatami M. 'Yin and Yang' in inflammation: duality in innate immune cell function and tumorigenesis. Expert Opin Biol Ther. 2008 Oct;8(10):1461-72.

355. Mantovani A. The yin-yang of tumor-associated neutrophils. Cancer Cell. 2009 Sep;16(3):173-4.

356. Saika S. Yin and yang in cytokine regulation of corneal wound healing: roles of TNF-alpha. Cornea. 2007 Oct;26(9 Suppl 1):S70-4.

357. Zhang J. Yin and yang interplay of IFN-gamma in inflammation and autoimmune disease. J Clin Invest. 2007 Apr;117(4):871-3. 
358. Mangano EN, Hayley S. Inflammatory priming of the substantia nigra influences the impact of later paraquat exposure: Neuroimmune sensitization of neurodegeneration. Neurobiol Aging. 2009 Sep;30(9):1361-78.

359. Raju U, Gumin GJ, Tofilon PJ. NF kappa B activity and target gene expression in the rat brain after one and two exposures to ionizing radiation. Radiat Oncol Investig. 1999 Jan; 7(3):145-52.

360. Kariko K, Weissman D, Welsh FA. Inhibition of toll-like receptor and cytokine signaling--a unifying theme in ischemic tolerance. J Cereb Blood Flow Metab. 2004 Nov;24(11):1288-304.

361. Dokladny K, Lobb R, Wharton W, Ma TY, Moseley PL. LPS-induced cytokine levels are repressed by elevated expression of HSP70 in rats: possible role of NFkappaB. Cell Stress Chaperones. 2010 Mar;15(2):153-63.

362. Ghosh S, May MJ, Kopp EB. NF-kappa B and Rel proteins: evolutionarily conserved mediators of immune responses. Annu Rev Immunol. 1998 Jan;16:22560.

363. Hayden MS, Ghosh S. Signaling to NF-kappaB. Genes Dev. 2004 Sep;18(18):2195-224.

364. Karin M, Greten FR. NF-kappaB: linking inflammation and immunity to cancer development and progression. Nat Rev Immunol. 2005 Oct;5(10):749-59.

365. Manna SK, Mukhopadhyay A, Van NT, Aggarwal BB. Silymarin suppresses TNF-induced activation of NF-kappa B, c-Jun N-terminal kinase, and apoptosis. J Immunol. 1999 Dec;163(12):6800-9.

366. Bui NT, Livolsi A, Peyron JF, Prehn JH. Activation of nuclear factor kappaB and Bcl-x survival gene expression by nerve growth factor requires tyrosine phosphorylation of IkappaBalpha. J Cell Biol. 2001 Feb;152(4):753-64.

367. Klintworth H, Garden G, Xia Z. Rotenone and paraquat do not directly activate microglia or induce inflammatory cytokine release. Neurosci Lett. 2009 Oct;462(1):1-5.

368. Berry C, La Vecchia C, Nicotera P. Paraquat and Parkinson's disease. Cell Death Differ. 2010 Jan:Epub ahead of print.

369. Hoglinger GU, Oertel WH, Hirsch EC. The rotenone model of parkinsonism-the five years inspection. J Neural Transm Suppl. 2006 Nov(70):269-72.

370. Pubmed. Searching by key word, "Parkinson's Disease". Pubmed; 2010.

371. Maines TR, Szretter KJ, Perrone L, Belser JA, Bright RA, Zeng H, et al. Pathogenesis of emerging avian influenza viruses in mammals and the host innate immune response. Immunol Rev. 2008 Oct;225:68-84.

372. Foley PB. Encephalitis lethargica and influenza. I. The role of the influenza virus in the influenza pandemic of 1918/1919. J Neural Transm. 2009 Feb;116(2):14350 .

373. Beigel JH, Farrar J, Han AM, Hayden FG, Hyer R, de Jong MD, et al. Avian influenza A (H5N1) infection in humans. N Engl J Med. 2005 Sep;353(13):137485.

374. Dhib-Jalbut S, Gogate N, Jiang H, Eisenberg H, Bergey G. Human microglia activate lymphoproliferative responses to recall viral antigens. J Neuroimmunol. 1996 Mar;65(1):67-73. 
375. Greenamyre JT, Sherer TB, Betarbet R, Panov AV. Complex I and Parkinson's disease. IUBMB Life. 2001 Sep;52(3-5):135-41.

376. Gomez C, Bandez MJ, Navarro A. Pesticides and impairment of mitochondrial function in relation with the parkinsonian syndrome. Front Biosci. 2007 Jan;12:1079-93.

377. Di Monte D, Sandy MS, Ekstrom G, Smith MT. Comparative studies on the mechanisms of paraquat and 1-methyl-4-phenylpyridine (MPP+) cytotoxicity. Biochem Biophys Res Commun. 1986 May;137(1):303-9.

378. Purisai MG, McCormack AL, Cumine S, Li J, Isla MZ, Di Monte DA. Microglial activation as a priming event leading to paraquat-induced dopaminergic cell degeneration. Neurobiol Dis. 2007 Feb;25(2):392-400.

379. Phinney AL, Andringa G, Bol JG, Wolters E, van Muiswinkel FL, van Dam AM, et al. Enhanced sensitivity of dopaminergic neurons to rotenone-induced toxicity with aging. Parkinsonism Relat Disord. 2006 May;12(4):228-38.

380. Hawkes CH, Del Tredici K, Braak H. Parkinson's disease: the dual hit theory revisited. Ann N Y Acad Sci. 2009 Jul;1170:615-22.

381. Matsuoka Y, Lamirande EW, Subbarao K. The ferret model for influenza. Curr Protoc Microbiol. 2009 May:UNIT 15G.2.

382. Matsuoka Y, Lamirande EW, Subbarao K. The mouse model for influenza. Curr Protoc Microbiol. 2009 May:UNIT 15G.3.

383. Lakadamyali M, Rust MJ, Zhuang X. Endocytosis of influenza viruses. Microbes Infect. 2004 Aug;6(10):929-36.

384. Momose F, Kikuchi Y, Komase K, Morikawa Y. Visualization of microtubulemediated transport of influenza viral progeny ribonucleoprotein. Microbes Infect. 2007 Oct;9(12-13):1422-33.

385. Mayer V, Schulman JL, Kilbourne ED. Nonlinkage of neurovirulence exclusively to viral hemagglutinin or neuraminidase in genetic recombinants of A-NWS (HON1) influenza virus. J Virol. 1973 Feb;11(2):272-8.

386. Hong JS. Role of inflammation in the pathogenesis of Parkinson's disease: models, mechanisms, and therapeutic interventions. Ann N Y Acad Sci. 2005 Aug; 1053:151-2.

387. Service AR. USDA security policies and procedures for biosafety level-3 facilities. In: Agriculture USDo, editor.: USDA; 2002.

388. Nishimura H, Itamura S, Iwasaki T, Kurata T, Tashiro M. Characterization of human influenza A (H5N1) virus infection in mice: neuro-, pneumo- and adipotropic infection. J Gen Virol. 2000 Oct;81(Pt 10):2503-10.

389. Schmidt J, Mertz K, Morgan JI. Regulation of heme oxygenase-1 expression by dopamine in cultured C6 glioma and primary astrocytes. Brain Res Mol Brain Res. 1999 Nov;73(1-2):50-9.

390. Yamato M, Kudo W, Shiba T, Yamada KI, Watanabe T, Utsumi H. Determination of reactive oxygen species associated with the degeneration of dopaminergic neurons during dopamine metabolism. Free Radic Res. 2010 Mar;44(3):249-57. 
391. Calabrese V, Mancuso C, Ravagna A, Perluigi M, Cini C, De Marco C, et al. In vivo induction of heat shock proteins in the substantia nigra following L-DOPA administration is associated with increased activity of mitochondrial complex I and nitrosative stress in rats: regulation by glutathione redox state. J Neurochem. 2007 May;101(3):709-17.

392. Hermida-Ameijeiras A, Mendez-Alvarez E, Sanchez-Iglesias S, Sanmartin-Suarez $\mathrm{C}$, Soto-Otero R. Autoxidation and MAO-mediated metabolism of dopamine as a potential cause of oxidative stress: role of ferrous and ferric ions. Neurochem Int. $2004 \mathrm{Jul} ; 45(1): 103-16$.

393. Karman J, Ling C, Sandor M, Fabry Z. Initiation of immune responses in brain is promoted by local dendritic cells. J Immunol. 2004 Aug;173(4):2353-61.

394. Yong VW, Marks S. The interplay between the immune and central nervous systems in neuronal injury. Neurology. 2010 Jan;74 Suppl 1:S9-S16.

395. Kuo TC, Schlissel MS. Mechanisms controlling expression of the RAG locus during lymphocyte development. Curr Opin Immunol. 2009 Apr;21(2):173-8.

396. Bruniquel D, Borie N, Hannier S, Triebel F. Regulation of expression of the human lymphocyte activation gene-3 (LAG-3) molecule, a ligand for MHC class II. Immunogenetics. 1998 Jul;48(2):116-24.

397. Laboratory TJ. Mouse model for cancer researches. 2008 [accessed 2010 May]; Available from: http://jaxmice.jax.org/literature/models/cancer.pdf

398. Rothwell NJ. Cytokines and acute neurodegeneration. Mol Psychiatry. 1997 Mar;2(2):120-1.

399. Geoffroy E. Contribution à l'étude du Robinia Nicou Aublet, au point de vue botanique, chimique et physiologique. Marseille: L'Institut Colonial de Marseille; 1895.

400. Michaelis L, Hill ES. The viologen indicators. Journal of General Physiology. 1933 Jan;16(6):859-73.

401. HSDB. Paraquat. In: Medicine NLo, editor.: National Library of Medicine; 1992.

402. Di Monte DA. The environment and Parkinson's disease: is the nigrostriatal system preferentially targeted by neurotoxins? Lancet Neurol. 2003 Sep;2(9):5318. 


\section{VITA}

Haeman Jang was born in Eumsung, Chung-Buk province, Korea on December 20, 1974, the son of Teak Hyun Jang and Hyun Nam Kyung. Haeman obtained his bachelor's and master's degree in Biochemistry and Molecular Biology from Hanyang University in 2000 and 2003. In 2003, he joined Dr. Michael Levin's lab in the Department of Neurology, University of Tennessee Health Science Center as a research technician. In 2005, he began his graduate studies at the University of Tennessee Health Science Center and joined Dr. Richard Smeyne's lab in the Department of Developmental Neurobiology at St. Jude Children's Research Hospital. Haeman was honored with Congress Travel Award from XVIII WFN World Congress on Parkinson's Disease and Related Disorders. Haeman received his Doctor of Philosophy degree from the University of Tennessee Health Science Center in May of 2010.

\section{PUBLICATIONS}

Haeman Jang, David Boltz, Jennifer McClerran, Robert Webster and Richard Jay Smeyne. Prior H5N1 Influenza Virus infection yield protection from paraquat-induced toxicity in mice. In preparation.

Haeman Jang, David Boltz, Amar Pani, Jennifer McClerran, Robert Webster and Richard Jay Smeyne. Inflammatory effects of highly pathogenic H5N1 influenza viral infection in the CNS. In preparation.

Haeman Jang, David Boltz, Katharine Sturm-Ramirez, Kennie R. Shepherd, Yun Jiao, Robert Webster and Richard J. Smeyne. Highly pathogenic H5N1 influenza virus can enter the central nervous system and induce neuroinflammation and neurodegeneration. PNAS. 2009, Aug;106(33):14063-8.

Jang H, Boltz DA, Webster RG, Smeyne RJ. Viral parkinsonism. Biochim Biophys Acta. 2009, Aug;1792:714-721.

Boyd JD*, Jang H*, Shepherd KR, Faherty C, Slack S, Jiao Y, Smeyne RJ. Response to 1-methyl-4-phenyl-1,2,3,6-tetrahydropyridine (MPTP) differs in mouse strains and reveals a divergence in JNK signaling and COX-2 induction prior to loss of neurons in the substantia nigra pars compacta. Brain Res. 2007, Oct;1175:107-16. (* Equal contribution). 